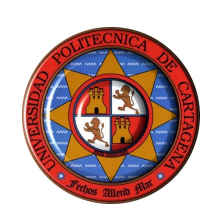

Universidad Politécnica de Cartagena

Departamento de Física Aplicada

Grupo de Astrofísica y Materia Condensada

\title{
Transporte Electrónico en Nanoestructuras de Carbono
}

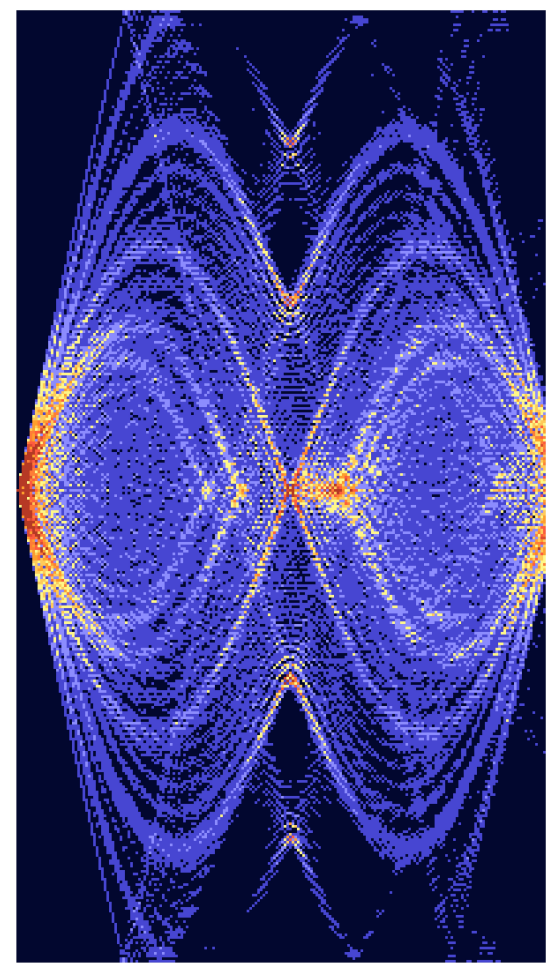

TESIS DOCTORAL DE ESTHER JÓDAR FERRÁNDEZ 



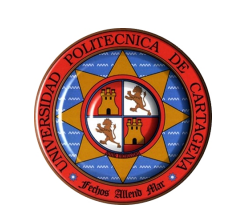

Universidad Politécnica de Cartagena

Departamento de Física Aplicada

Grupo de Astrofísica y Materia Condensada

\section{Transporte Electrónico en Nanoestructuras de Carbono}

Directores:

Dr. Antonio Pérez Garrido

Dr. Vladimir Gasparian
Memoria presentada por Esther Jódar Ferrández para optar al Grado de Doctor por la UPCT. Cartagena, julio de 2007 

"No se ve bien sino con el corazón, lo esencial es invisible a los ojos..."

A Pedro, a mis padres

y a mis hermanos. 



\section{Agradecimientos}

Quisiera expresar mi agradecimiento:

Al profesor Antonio Pérez Garrido, por su gran dedicación y minuciosidad en la dirección de todo mi trabajo. Sobre todo por su amistad.

Al profesor Vladimir Gasparian, por tutelar mi trabajo a lo largo de todos estos años. Por su apoyo incondicional y por su ayuda en los momentos difíciles.

A los miembros del grupo de investigación de Astrofísica y Materia Condensada de la Universidad Politécnica de Cartagena, por haberme acogido e integrado en su grupo. Especialmente a Anastasio Díaz y José Damián Catalá.

A todos los miembros del departamento de Física Aplicada de la Universidad Politécnica de Cartagena, por brindarme su apoyo y su experiencia académica en todo momento. En especial a Paco Alhama y Carlos F. González.

Al profesor Antonio Urbina y a Santiago Heredia, que me han acompañado en muchas ocasiones a lo largo de este camino.

Al SAIT de la Universidad Politécnica de Cartagena, en particular a Luis Pedro García, por su gran profesionalidad. Y claro está, a Prometeo. 

Publicaciones relacionadas con el trabajo de la tesis:

1. V. Gasparian, Ute Gummich, E. Jódar, J. Ruiz y M. Ortuño, Tunneling and Dwell Time for One-Dimensional generalized Kronig-Penney Model., Physica B. Condensed Matter 233, 72 (1997).

2. E. Jódar, A. Pérez-Garrido y A. Díaz-Sánchez, Resonant cavities in metallic single-wall nanotubes: Green's function calculation., Physical Review B 73, 205403 (2006).

3. E. Jódar y A. Pérez-Garrido, Transmission and localization in cavities of nanotubes, Physica Status Solidi (a) 204, 1892 (2007).

4. J. Ruiz, E. Jódar y V. Gasparian, Global Partial density of states: Statistics and localization length in quasi-one-dimensional disordered wires., Physical Review B 75, 235123 (2007).

5. J. Ruiz, V. Gasparian y E. Jódar, Global Partial density of states in quasi-one dimensional disordered systems, Proceedings of the International Conference on Semiconductor Micro and Nano-electronics (Yerevan, Armenia) (Aceptado) (2007).

6. E. Jódar, A. Pérez-Garrido y Fernando Rojas, Bloch oscillations in Carbon Nanotubes, Physical Review Letters (Enviado) (2007).

7. E. Jódar, A. Pérez-Garrido y Fernando Rojas, Bloch oscillations and confinement in cavities of Nanotubes, Physica Status Solidi (a) (Enviado) (2007). 



\section{Resumen}

El trabajo desarrollado a lo largo de la presente memoria se centra en el estudio de propiedades asociadas al transporte electrónico en diversas estructuras formadas por nanotubos de carbono. Esta memoria está dividida en cuatro capítulos cuyo contenido resumimos de manera muy breve en los siguientes puntos:

$\square$ Capítulo 1: Nanotubos de carbono. En el primer capítulo damos un repaso a las características y propiedades de los nanotubos de carbono. Se realiza aquí una introducción que nos permitirá entender posteriormente el comportamiento de los sistemas objeto de estudio en este trabajo: estructuras formadas por nanotubos de carbono.

En primer lugar, realizamos una revisión de la historia que condujo al descubrimiento de los nanotubos de carbono, pasando por el hallazgo previo de los fullerenos. También hacemos una revisión de los procedimientos experimentales más básicos que permiten sintetizarlos. La parte más importante de este capítulo es la descripción de la geometría, propiedades y estructura electrónica de los nanotubos de carbono. Se utilizarán a lo largo de los siguientes capítulos muchas de las expresiones que aquí se exponen. Finalmente, tratamos brevemente en este capítulo algunos de los campos de investigación más actuales relacionados con los nanotubos de carbono. 
$\square$ Capítulo 2: Método de cálculo. En este capítulo nos centramos en la descripción de los métodos de cálculo utilizados en esta memoria. Para ello se realiza previamente una introducción teórica y un breve desarrollo de los métodos que conducen a la obtención de las expresiones utilizadas. Ocupa buena parte de este capítulo la descripción de la función de Green, ya que muchos de los cálculos realizados a lo largo de este trabajo se basan en el conocimiento de esta función para el sistema bajo estudio. Se finaliza este capítulo con la aplicación del método teórico al cálculo de propiedades de nanotubos de carbono puros como ejemplo de uso de estos métodos. En cualquier caso, estos resultados previos nos servirán de base para capítulos posteriores.

$\square$ Capítulo 3: Estudio de cavidades de CNT. En general, llamamos cavidades de nanotubos a estructuras formadas cuando un nanotubo finito se une por ambos extremos a dos nanotubos de diferente geometría, de manera que el diámetro central de la estructura final es mayor que el de los dos extremos. Llevamos a cabo en este capítulo un estudio sistemático de las propiedades asociadas a las cavidades como la función de transmisión o la densidad local de estados para diversas estructuras. Los cálculos efectuados nos permiten conocer la dependencia de las propiedades obtenidas con diversos parámetros asociados a la cavidad. Entre estos parámetros destacamos el diámetro y la separación entre las cavidades (en el caso del acoplamiento entre varias). En nuestro estudio obtenemos diferentes resultados que han sido publicados en revistas internacionales del área (Jódar et al. 2006, Jódar y Pérez-Garrido 2007). Entre estos resultados queremos resaltar el hallazgo de estados cuasi-localizados en las cavidades de nanotubos que afectan a la función de transmisión, el comportamiento de las cavidades como puntos cuánticos cuando se unen nanotubos metálicos con semiconductores, y el estudio de la evolución de los sistemas de múltiples cavidades al límite de infinitas cavidades. 
$\square$ Capítulo 4: Oscilaciones de Bloch. Este capítulo está dedicado al estudio del comportamiento de los electrones en nanotubos de carbono en presencia de un campo eléctrico constante unidimensional. En particular, nos centramos en la caracterización de las oscilaciones que realiza la función de onda (conocidas como oscilaciones de Bloch). Además, analizamos la probabilidad de ocupación y el desplazamiento cuadrático medio. Es de resaltar el hecho de que no hemos encontrado en la bibliografía referencia alguna que hable acerca de las oscilaciones de Bloch en nanotubos de carbono.

Comenzamos realizando los cálculos para nanotubos de carbono puros de diferentes longitudes y valores del campo eléctrico, encontrando una clara correspondencia de la frecuencia de Bloch teórica correspondiente a cadenas unidimensionales de átomos con la obtenida con nuestro cálculo. Además, mostramos el diferente comportamiento de funciones de onda localizadas y extendidas. Finalizamos este capítulo aplicando el campo eléctrico a las cavidades estudiadas en el capítulo 3. En este caso se encuentra un resultado especialmente interesante: puede conseguirse el confinamiento de la onda en diferentes regiones mediante la aplicación de un campo eléctrico en una o varias regiones de la estructura. 



\section{Summary}

The aim of this work is the study of the electronic transport properties in several structures made of carbon nanotubes. This dissertation is divided in four chapters:

$\square$ Chapter 1: Carbon Nanotubes. This chapter is a brief review of the foundations of carbon nanotubes (CNT). Main properties of CNT are explained. The subject developed here is important for the understanding of the results obtained in the bulk of this thesis.

We carry out, in the first part of this chapter, an historical review of the discovering of CNT, that includes the history of the discovering of fullerenes, the predecessors for carbon nanotubes. Afterwards, a revision of the different methods for synthesizing nanotubes is done. The main part of this chapter treats of the description of the geometry, properties and electronic structure of CNT. Many equations deduced here will be used later. Finally, we discuss some research lines related to carbon nanotubes.

$\square$ Chapter 2: Theoretical and numerical method. In this chapter we describe the numerical method we have developed to obtain the results presented in this work. For this purpose it is necessary to describe previously the theoretical method on which our calculations are based. We extensively explain the Green's function and its properties. A large part of our calculations are based 
in the obtention the GF of the system under study. This chapter finishes with the application of the equations described in order to obtain electronic properties associated with pure carbon nanotubes as an example of use. Anyway, these previous results will be used later.

$\square$ Chapter 3: Cavities made of nanotubes. We denote as a cavity to the structure formed with a carbon nanotube sandwiched between other two carbon nanotubes (contacts), provided that the central region is wider than these contacts. In this chapter we perform some calculations of the properties associated to the electronic transport in cavities, as the local density of states and the transmission function. We analyze the influence of the width of the cavity and the distance between them (in the case of multiple cavities). Some interesting results are obtained in these calculations which have been published in international journals (Jódar et al. 2006, Jódar y Pérez-Garrido 2007). We emphasize the presence of quasi-localized states in the cavities, which affects to the transmission function, the behaviour of some cavities formed with semi-conductor as quantum dots, or the study of the evolution of the system with multiple cavities to the limit of infinite cavities.

$\square$ Chapter 4: Bloch Oscillations. In this chapter we investigate the properties of carbon nanotubes under a constant electric field. This configuration show Bloch oscillations, according to the work of Bloch and Zener. We study here the dynamics of these oscillations for different geometries as a function of the electric field applied. Specially, the behaviour of the occupation probability and the averaged quadratic displacement as a function of time. We have not found bibliography that deals with this phenomenon in Carbon Nanotubes, which is the aim of this chapter.

We first study the behaviour of electrons in pure carbon nanotubes in a constant electric field, for different lengths of the CNT and different values of the 
electric field applied. We show how wavefunctions oscillate with a period that coincides with that given by theoretical expressions of Bloch oscillations for linear chains of atoms. Besides, we show the different kind of behaviour of localized and extended waves. In the final part of this chapter we apply a constant electric field to the structure studied in the chapter 3, i. e., the cavity. We show in this case that, besides Bloch oscillations, electrons can be confined in certain regions only by inserting the nanotube in an electric field. 


\section{Índice general}

$\begin{array}{ll}\text { Introducción } & 1\end{array}$

$\begin{array}{ll}\text { 1. Nanotubos de carbono } & 7\end{array}$

1.1. Antecedentes históricos .................. 11

1.2. Fullerenos ......................... 13

1.3. Síntesis de nanotubos de carbono . . . . . . . . . . . . . 20

1.4. Geometría y caracterización de los nanotubos de carbono . . . . . . 28

1.5. Estructura electrónica . . . . . . . . . . . . . 34

1.6. Líneas de investigación . . . . . . . . . . . . . . 45

1.6.1. Propiedades electrónicas . . . . . . . . . . . 46

1.6.2. Propiedades mecánicas . . . . . . . . . . . . 47

1.7. Puntos cuánticos ..................... 48

2. Método de cálculo $\quad 51$

2.1. Modelo tight-binding para sólidos cristalinos . . . . . . . . . 52

2.1.1. Aplicación a CNT. Función de participación . . . . . . . . 55

2.2. Función de Green. Definición . . . . . . . . . . . . . 58 
2.2.1. Relación con las funciones de onda . . . . . . . . . . . 62

2.2.2. Aplicación a sistemas finitos. Autoenergías . . . . . . . 63

2.2.3. Sistemas multi-modos ................ 65

2.3. Función de Transmisión y Densidad de Estados . . . . . . . . . 67

2.4. Método numérico ..................... 71

2.5. Aplicación a CNT puros e infinitos ............ 73

2.5.1. Función de transmisión .............. 73

2.5.2. Densidad de estados . . . . . . . . . . . . 77

3. Estudio de Cavidades de CNT $\quad 83$

3.1. Descripción de las cavidades de nanotubos . . . . . . . . . 84

3.2. Estudio de la cavidad $(6,6) / 4(9,9) /(6,6) \ldots \ldots \ldots 6$

3.2.1. Función de transmisión . . . . . . . . . . . . 87

3.2.2. Densidad de estados . . . . . . . . . . . . 92

3.2.3. Función de participación y funciones de onda . . . . . . . 95

3.3. Influencia del diámetro de la cavidad en la función de transmisión . 101

3.4. Cavidades de semiconductores . . . . . . . . . . . . 104

3.4.1. Cavidad $(5,4) / 4(6,6) /(5,4) \ldots \ldots . \ldots . \ldots 105$

3.4.2. Cavidad $(5,2) / 5(6,6) /(5,4) \ldots \ldots . \ldots 110$

3.5. Estudio de un estrechamiento: nanoestructura $(9,9) / 4(6,6) /(9,9) \quad$. 116

3.6. Acoplamiento de cavidades. Red infinita . . . . . . . . . . . 120 
4.1. Fundamento teórico . . . . . . . . . . . . . . . . . . 128

4.2. Método de cálculo . . . . . . . . . . . . . . . . . . . . . . 131

4.2.1. La transformada de Fourier . . . . . . . . . . . . 136

4.3. Oscilaciones de Bloch en nanotubos puros. Ondas de Wannier . . . . 137

4.3.1. Funciones de onda. Probabilidad de ocupación . . . . . . . . 137

4.3.2. Análisis de la oscilación . . . . . . . . . . . . . . . 146

4.3.3. Difusión transversal al campo eléctrico . . . . . . . . . . 151

4.3.4. Desplazamiento cuadrático medio . . . . . . . . . . . 156

4.4. Oscilaciones de Bloch en nanotubos puros. Ondas extendidas . . . . 160

4.4.1. Funciones de onda. Probabilidad de ocupación . . . . . . . 162

4.4.2. Desplazamiento cuadrático medio . . . . . . . . . . 166

4.4.3. Función de participación . . . . . . . . . . . . . 170

4.5. Estudio de las oscilaciones de Bloch en cavidades . . . . . . . . . 173

4.5.1. Campo eléctrico en el interior de la cavidad . . . . . . . . . 173

4.5.2. Campo eléctrico en los contactos . . . . . . . . . . 177

4.5.3. Campo eléctrico en toda la estructura . . . . . . . . . . . 179

$\begin{array}{ll}\text { Conclusiones } & 181\end{array}$

$\begin{array}{ll}\text { Bibliografía } & 184\end{array}$ 


\section{Introducción}

Los nanotubos de carbono fueron descubiertos por S. Iijima, que publicó su descubrimiento en la prestigiosa revista Nature en 1991. Básicamente se pueden considerar como láminas de grafeno enrolladas sobre sí mismas. El grafeno es cada una de las capas bidimensionales de átomos de carbono cuyo apilamiento da lugar al grafito. Tienen un diámetro de unos pocos nanometros, mientras que su longitud puede ser de hasta unos pocos milímetros. Desde su descubrimiento, el estudio teórico y experimental de sus propiedades se ha convertido en uno de los campos más productivos de la física. Esto es debido principalmente a dos factores: en primer lugar, la gran cantidad de geometrías diferentes que forman el conjunto de los nanotubos, con propiedades muy diversas. En segundo lugar, las excelentes propiedades, tanto mecánicas como electrónicas que estos poseen y que han despertado grandes expectativas en torno a las posibles aplicaciones prácticas de los mismos. Los nanotubos de carbono son moléculas con unas propiedades mecánicas muy interesantes debido a su gran resistencia y elasticidad, por lo que ya se investigan sus posibles aplicaciones en la industria automovilística y aeroespacial. Además, algo que hace únicas a estas moléculas es que pueden tener diferentes propiedades de transporte electrónico al cambiar su geometría. En otras palabras, podemos tener nanotubos metálicos o semiconductores simplemente cambiando la disposición de los átomos en la red. Esto hace de ellos excelentes candidatos para el desarrollo de dispositivos electrónicos más pequeños y versátiles que los actuales. Actualmente ya se han conseguido producir transistores de nanotubos, lo que parece la antesala del 
desarrollo de una nueva nanoelectrónica. Además, se han encontrado recientemente ciertas aplicaciones de los nanotubos de carbono a la medicina, que podrían ser utilizados como sondas químicas o genéticas. Sin embargo, aunque los métodos de fabricación de nanotubos de carbono se han desarrollado enormemente a lo largo de los últimos años, todavía no es posible su fabricación con un nivel de control suficiente que permita obtener las geometrías deseadas.

El cálculo llevado a cabo en esta memoria sobre nanoestructuras de carbono se basa en la aplicación de un modelo de enlace fuerte (tight binding), en el cual se considera que existe únicamente interacción entre los átomos del nanotubo de carbono que están unidos por medio de un enlace covalente (lo que llamaremos vecinos próximos). Dentro de este modelo se considera, además, que la función de onda del sistema se expresa como un conjunto de orbitales atómicos localizados, uno por cada sitio. Supondremos también que los átomos poseen un único orbital $\pi$. El cálculo de las propiedades de transmisión se lleva a cabo con diferente metodología en los dos grupos de resultados de que consta esta memoria. En la primera parte, se obtendrá la función de transmisión, así como la densidad de estados en nanoestructuras de cavidades y estrechamientos a través del cálculo de la función de Green del sistema. En la segunda parte, se obtendrá el desplazamiento cuadrático medio de las funciones de onda en presencia de un campo eléctrico actuando sobre el nanotubo a través del cálculo de las autofunciones del Hamiltoniano del sistema.

Como objetivos principales de esta memoria consideramos los siguientes aspectos:

$\square$ Obtener numéricamente geometrías de nanoestructuras de carbono mediante uniones entre diferentes tipos de nanotubos, de manera que se produzcan cavidades y estrechamientos en las mismas, para así calcular la función de transmisión y la densidad local de estados en estas nanoestructuras.

$\square$ Investigar la generación de estados localizados en nanoestructuras con cavidades, calculando el radio de participación de las funciones de onda electrónicas. 
$\square$ Estudiar cómo las cavidades y estrechamientos afectan a la transmisión electrónica.

$\square$ Calcular la evolución de la función de transmisión con el número de cavidades.

$\square$ Estudiar las peculiaridades de las cavidades formadas con nanotubos semiconductores, como la aparición de características similares a las de los puntos cuánticos.

$\square$ Investigar la generación de oscilaciones de Bloch en nanotubos puros de carbono sometidos a un campo eléctrico constante.

$\square$ Calcular el desplazamiento cuadrático medio y la función de participación de las funciones de onda en los nanotubos sometidos a un campo eléctrico.

$\square$ Estudiar la dependencia de la frecuencia de las oscilaciones de Bloch con el valor del campo eléctrico.

$\square$ Resaltar el diferente comportamiento de las funciones de onda localizadas y extendidas en presencia del campo eléctrico.

$\square$ Aplicar el formalismo anterior al estudio del comportamiento de las autofunciones de onda en cavidades de nanotubos sometidas a campos eléctricos en diferentes regiones del mismo.

El trabajo realizado durante estos años, en los que hemos tratado de cubrir los objetivos antes expuestos, lo podemos dividir en dos categorías, como antes hemos mencionado:

$\square$ En primer lugar, realizamos el estudio de sistemas compuestos por uniones entre nanotubos de diferente geometría y estructura electrónica, que dan lugar a cavidades y estrechamientos que afectan enormemente a la conducción a lo 
largo de las mismas. En este tipo de sistemas se producen fenómenos cuánticos de diversa índole que se exponen y que justificamos convenientemente a lo largo de esta memoria.

$\square$ En segundo lugar, analizamos los fenómenos dinámicos asociados a la introducción de un campo eléctrico constante en nanotubos de carbono, calculando la dependencia temporal de las funciones de onda asociadas a dicha estructura. La inserción de un campo eléctrico constante en un potencial periódico da lugar a oscilaciones del paquete de ondas, llamadas oscilaciones de Bloch. Sin embargo, no existe bibliografía hasta el momento que trate este fenómeno en nanotubos de carbono. Realizaremos aquí un análisis exhaustivo de dichas oscilaciones, estudiando la dependencia de la frecuencia de la oscilación con el valor del campo eléctrico.

La memoria está dividida en cuatro capítulos. En el primero de ellos se explican las características generales de los nanotubos de carbono, así como sus propiedades más relevantes, con el fin de que sirvan de base a los resultados que posteriormente obtendremos para el cálculo en estructuras más complejas. Hacemos también un breve repaso a la historia de su descubrimiento, así como a los métodos experimentales de síntesis y los últimos avances científicos en torno a los mismos.

En el segundo capítulo exponemos el método de cálculo que hemos implementado, así como las bases teóricas utilizadas en nuestro trabajo. Se trata de presentar diversos conceptos básicos que se utilizarán en el resto de capítulos de esta memoria, como la obtención de la función de Green a partir de la cual calculamos la función de transmisión y la densidad de estados, o el cálculo de las autofunciones de onda.

El tercer capítulo está dedicado por completo a presentar los resultados de la aplicación de nuestro cálculo a diversas geometrías de nanotubos de carbono. En particular, usaremos uniones formadas por nanotubos de diferente diámetro. Se mostrarán las funciones de transmisión y densidades locales de estados de dichas 
estructuras, y se estudiarán los fenómenos característicos de las mismas, como la aparición de interferencias y estados cuasi-localizados en cavidades y estrechamientos y el posible comportamiento como puntos cuánticos.

Por último, dedicamos el cuarto capítulo de esta memoria al estudio de la dinámica de las funciones de onda en nanotubos sometidos a campos eléctricos constantes. Calcularemos la dependencia temporal de ondas localizadas y extendidas en los mismos, con el fin de observar el comportamiento de las oscilaciones de Bloch producidas. Así mismo, realizaremos un estudio de la dependencia de dichas oscilaciones con el valor del campo eléctrico, y veremos cómo estos campos pueden confinar electrones en determinadas regiones de nanotubos donde haya cavidades. Finalizamos esta memoria con las conclusiones de este trabajo. 


\section{Capítulo 1}

\section{Nanotubos de carbono}

El estudio llevado a cabo en esta memoria está dedicado a las propiedades de transporte electrónico en nanotubos de carbono, carbon nanotubes (CNT), de diferentes geometrías. Así pues, dedicaremos este primer capítulo al repaso de algunos conceptos generales sobre dichas estructuras. Existe numerosa bibliografía sobre nanotubos de carbono que puede consultarse para una introducción más amplia acerca de los mismos (Saito et al. 1998, Dresselhaus et al. 2001).

Como sabemos, el grafito está formado por varias capas de redes hexagonales planas de átomos de carbono, donde cada átomo está enlazado a otros tres. Cada una de estas capas recibe el nombre de grafeno. Los nanotubos de carbono son moléculas con geometría cilíndrica formadas únicamente por átomos de carbono. Podemos visualizar su estructura suponiéndola formada a partir de láminas de grafeno. Puede verse una representación esquemática del mismo en la figura 1.1. Tomando un rectángulo de una lámina de grafeno y enrollándolo sobre sí mismo hasta formar un cilindro obtenemos la geometría básica de un nanotubo de carbono, aunque evidentemente el proceso de fabricación de los nanotubos no es éste. 


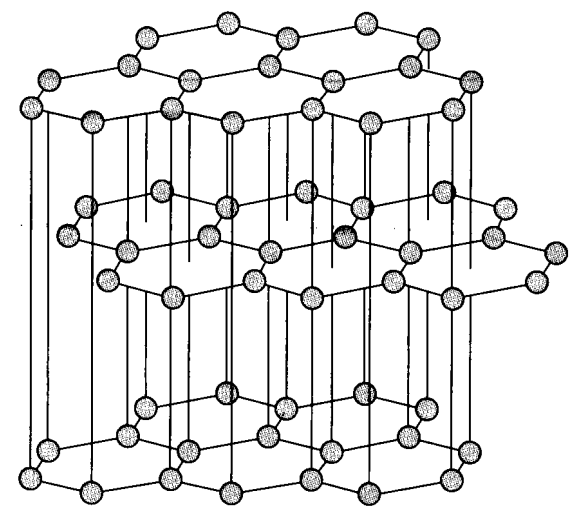

Figura 1.1: Representación esquemática del grafito. Los átomos de carbono se representan mediante círculos sombreados. En la figura se representan 3 capas de grafeno. Se observa la estructura plana de cada capa de grafeno formando una red hexagonal. La distancia entre capas es de $3,35 \AA$, por lo que la interacción entre ellas es débil. La distancia entre los átomos de carbono de una sóla capa es de 1,42 $\AA$.

Los nanotubos formados por una sola capa de grafeno se conocen como SWNT (single-wall nanotube). También se pueden sintetizar nanotubos formados por más de una capa de grafeno enrolladas coaxialmente. Estos últimos reciben el nombre de nanotubos multicapa, multi-wall nanotube (MWNT). En la figura 1.2 podemos ver una representación de estos dos tipos de nanotubos.

Los diámetros típicos de los SWNT son del orden de 1-2 nm, mucho menores que su longitud (que puede llegar a ser de varios milímetros, aunque típicamente es de orden micrométrico), por lo que pueden ser considerados como estructuras cuasi-unidimensionales. Esta geometría en forma de cilindro hace que todos posean simetría axial. Sin embargo, existen muchos tipos diferentes de nanotubos con propiedades radicalmente opuestas. Es la única estructura descubierta hasta el momento que puede presentar carácter metálico o semiconductor dependiendo de su geometría. En concreto, las propiedades de conducción dependen de la forma en 

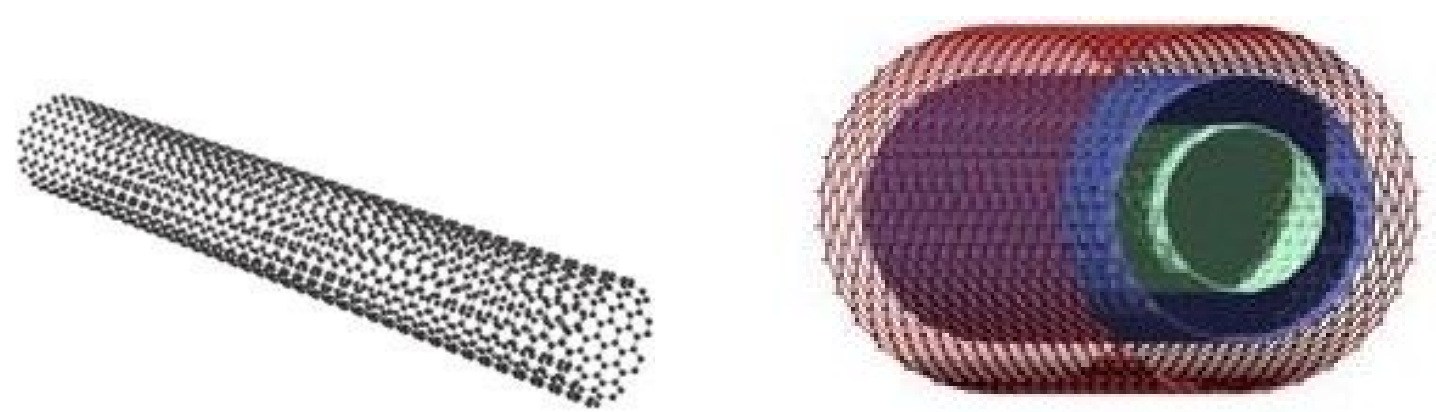

Figura 1.2: Representación de los dos tipos principales de nanotubos. A la izquierda, un nanotubo de una sóla capa (SWNT). A la derecha, un nanotubo multicapa (MWNT) compuesto por tres capas representadas en verde, azul y rojo.

que se distribuyen los hexágonos formados por los enlaces de los átomos de carbono con respecto al eje del nanotubo. Esta distribución se mide a través de un factor asociado a la geometría del nanotubo denominado vector quiral. Así, hablamos de tres tipos básicos de nanotubos: armchair, zigzag y quiral, representados en la figura 1.3. Ampliaremos este concepto en el apartado 1.4.

El descubrimiento de los CNT condujo a la búsqueda de nanoestructuras formadas por átomos o moléculas diferentes de carbono, pero que como éste tuvieran la propiedad de formar redes planas similares a la del grafito. Así, hoy en día se conocen diferentes tipos de redes de moléculas inorgánicas a partir de las cuales se pueden sintetizar nanotubos, y los descubrimientos de nuevos tipos se suceden a gran velocidad. Entre los primeros nanotubos inorgánicos que se descubrieron se encuentran los formados por $\mathrm{WS}_{2}, \mathrm{MoS}_{2}, \mathrm{BN}$ y $\mathrm{NbS}_{2}$. Su estructura atómica es igual a la de los CNT. Sin embargo, sus propiedades mecánicas y electrónicas varían de un tipo a otro, siendo en algunas contadas excepciones superiores a las de los CNT (Terrones et al. 1996, Tenne et al. 1998). 


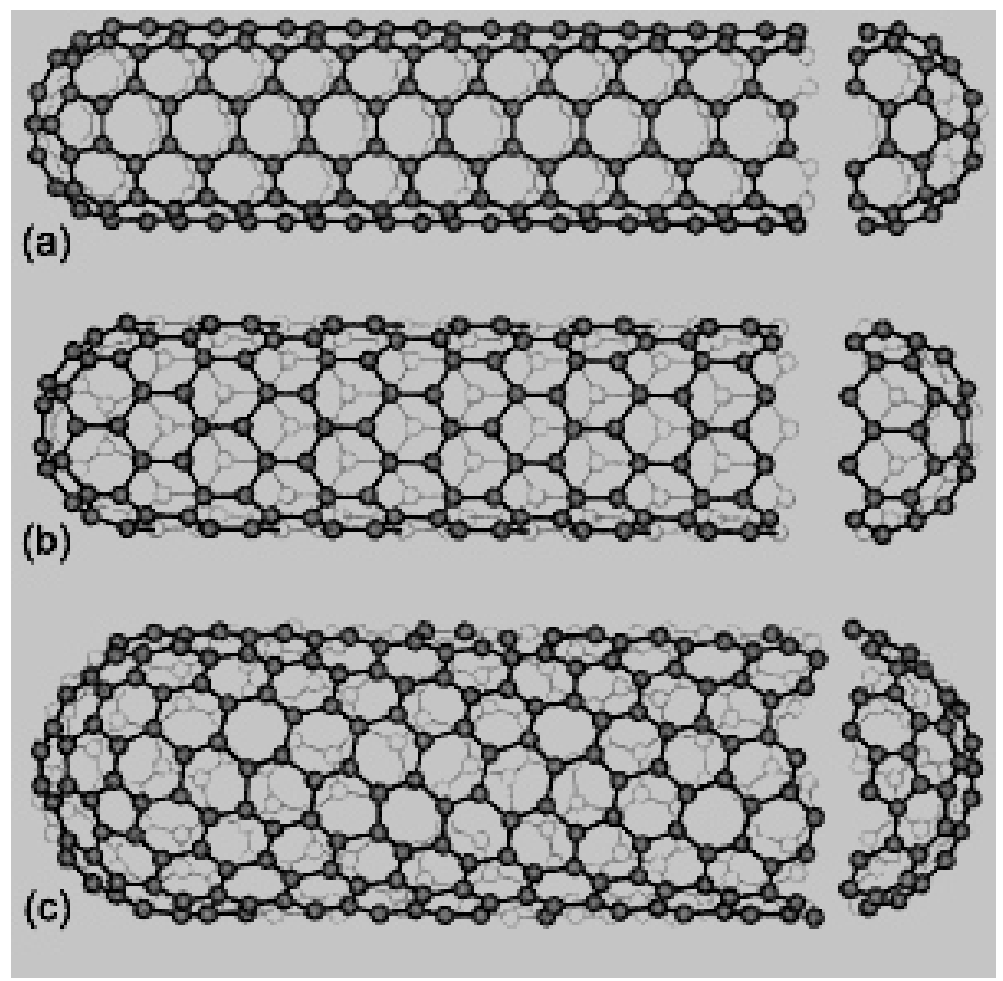

Figura 1.3: Representación de tres nanotubos con diferente quiralidad. La figura a) representa un nanotubo tipo armchair, la b) un nanotubo tipo zigzag y la c) uno con geometría quiral. Resultan muy significativas las diferencias entre las tres geometrías, especialmente si nos fijamos en los bordes de cada una de ellas. 


\subsection{Antecedentes históricos}

El estudio y la fabricación de fibras de carbono puede considerarse el antecedente histórico de los nanotubos de carbono para el caso de estructuras macroscópicas. Thomas A. Edison fabricó en 1878 el primer filamento de carbono para lo que sería el prototipo de bombilla eléctrica. Sin embargo, cuando los filamentos de carbono fueron sustituidos por los de tungsteno, la investigación en fibras de carbono quedó relegada a un segundo plano. A partir del año 1950 las industrias aeronáutica y aeroespacial comenzaron a desarrollar un estudio sistemático de nuevos materiales con el fin de encontrar materiales ligeros con buenas propiedades mecánicas. Esto provocó nuevamente un avance en la investigación de propiedades de materiales compuestos por fibras de carbono, muy ligeros, así como el desarrollo de técnicas de fabricación de los mismos (Moore et al. 1962, Blackman y Ubbelohde 1962).

La principal dificultad en la producción de fibras de carbono vino al tratar de reducir el número de defectos producidos para conseguir una mayor resistencia de las mismas. Así, a partir de 1960 comenzaron a desarrollarse procesos de síntesis de fibras de carbono mediante la descomposición de hidrocarburos a altas temperaturas (Koyama 1972, Endo et al. 1976, Tibbetts 1983, Tibbetts 1984). Cuando los procesos de fabricación ya se encontraban bastante desarrollados, comenzó a observarse que aparecían ocasionalmente estructuras de fibras micrométricas (Oberlin et al. 1976a, Oberlin et al. 1976b), pero no se realizó un estudio sistemático de las propiedades de dichas estructuras.

Los nanotubos se descubrieron gracias a la aparición previa de los fullerenos en 1985. Los fullerenos fueron vistos por primera vez por Kroto y su equipo de la Universidad de Rice en Houston (Kroto et al. 1985), lo que les valió para recibir el premio Nobel de química del año 1996. Este descubrimiento fue el que provocó que comenzaran a estudiarse de forma sistemática las propiedades de las estructuras moleculares de carbono. Los fullerenos son básicamente moléculas en las cuales 
los enlaces entre átomos de carbono se producen en la misma forma que en el grafeno pero con una topología esférica, formando parte de la misma cierto número de pentágonos e incluso heptágonos. Los pentágonos introducen un cierto ángulo de curvatura, que hace que la estructura sea cerrada (Figura 1.4). Dada su importancia, hablaremos de estas moléculas detalladamente en el apartado 1.2.

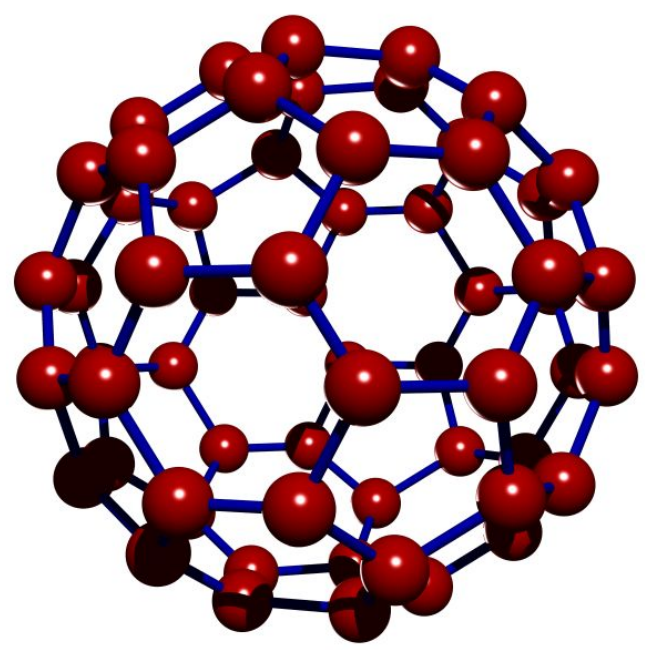

Figura 1.4: Representación del fullereno $C_{60}$. Fue el primero en ser descubierto, aunque posteriormente se encontrarían muchos tipos más. Los átomos de carbono se enlazan de manera que para formar la estructura cerrada se forman algunos pentágonos además de los hexágonos típicos del grafeno.

Iijima fue el primero en observar experimentalmente nanotubos de carbono utilizando un microscopio electrónico de alta resolución mientras sintetizaba fullerenos (Iijima 1991). Estas nuevas moléculas suscitaron gran interés en la comunidad científica y se crearon multitud de líneas de investigación sobre sus propiedades, tanto a nivel teórico como experimental. Este interés dentro de la comunidad científica es debido principalmente a dos factores: en primer lugar el tamaño de los CNT, mucho menor que la mayoría de los dispositivos electrónicos actuales. En segundo lugar su estructura electrónica, que les permite comportarse como metales o semiconduc- 
tores dependiendo de la geometría del propio nanotubo, como hemos mencionado anteriormente. Por este motivo, a los nanotubos de carbono se les prevén muchas aplicaciones experimentales de gran relevancia dentro de la física de los semiconductores.

\subsection{Fullerenos}

El descubrimiento de la existencia de los fullerenos en 1985 es uno de esos momentos que marcan la historia de la ciencia. Hasta entonces solamente se conocían dos formas alotrópicas cristalinas del carbono (grafito y diamante), lo cual resulta sorprendente si se tiene en cuenta que el carbono es uno de los elementos químicos más estudiados por ser el constituyente básico de los organismos vivos. De hecho, existen múltiples referencias que indican que sí había observaciones de fullerenos anteriores a su descubrimiento. La primera referencia conocida fue realizada 1970 (Osawa 1970). Aquellos que los vieron no los reconocieron por lo que eran: una forma nueva de carbono puro. No fue hasta 1985 en que estas moléculas de carbono fueron identificadas por un equipo de cinco químicos en el laboratorio del Departamento de Química de la Universidad de Rice en Houston (Kroto et al. 1985), lo que les valió a tres de ellos (Kroto, Curl y Smalley) el Premio Nobel de Química por su descubrimiento en 1996.

Para entender la historia que llevó al descubrimiento de los fullerenos hay que remontarse a la década de los setenta. El químico inglés H. Kroto investigaba en espectroscopía de microondas para analizar el gas en el espacio, tanto en atmósferas estelares como en nubes de gas interestelares. Utilizó la radioastronomía para capturar y analizar trazas de moléculas producidas en el espacio interestelar. En particular, centró sus investigaciones en detectar moléculas de cadena larga de carbono y nitrógeno (cianopolienos). Kroto suponía que estas cadenas se formaban en las atmósferas de estrellas gigantes ricas en carbono, así que buscó una forma de 
imitar las condiciones en que debían crearse.

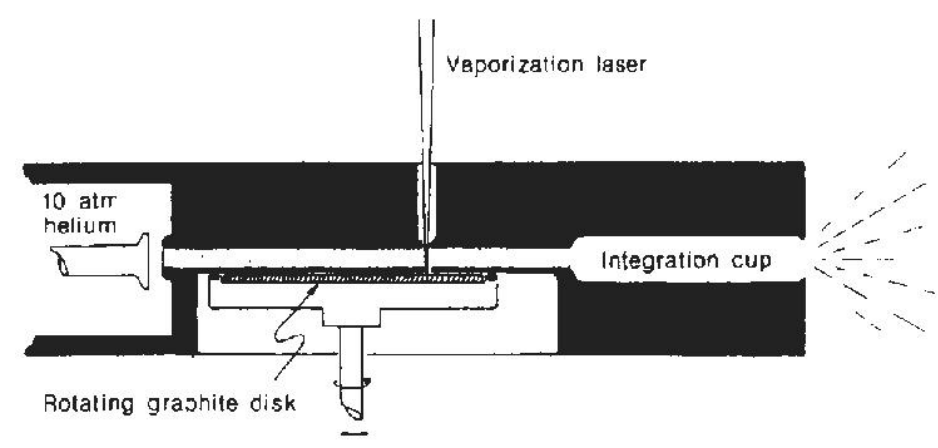

Figura 1.5: Dispositivo experimental diseñado por Smalley utilizado en la detección del $C_{60}$. La imagen muestra la ilustración original del artículo publicado en Nature.

Richard Smalley había diseñado una máquina que utilizaba junto con Robert Curl en la Universidad de Rice, en la que se podía vaporizar una barra de silicio. Para ello, se sometía a temperaturas que podían incluso alcanzar los diez mil grados. Por medio de un bombardeo mediante láser, se conseguía arrancar los átomos del silicio hasta convertirlos en un plasma. A continuación se lograba agrupar los átomos en una cámara de vacío mediante una nube de gas de Helio. Este gas, al ser inerte, no intervenía en la reacción. Kroto reemplazó el silicio que Curl y Smalley habían estado utilizando en sus experimentos por carbono. Al analizar los agregados de átomos de carbono que se encontraban en el plasma de la cámara de vacío, muy similares a los que componían el plasma estelar, encontró que existía una regularidad en el espectro de las cadenas de carbono. Una buena parte de las agrupaciones de átomos producidas contenían exactamente 60 átomos. Entonces el equipo formado por Kroto y sus ayudantes comenzó a preguntarse por la forma que tendrían estos conglomerados de 60 átomos. Como no podían quedar átomos con enlaces sueltos en los bordes, y tampoco podían unirse a nada ya que el conglomerado se encontraba 
en la cámara de vacío, les llevó algún tiempo encontrar la respuesta a esta pregunta, que casi fue hallada por casualidad.

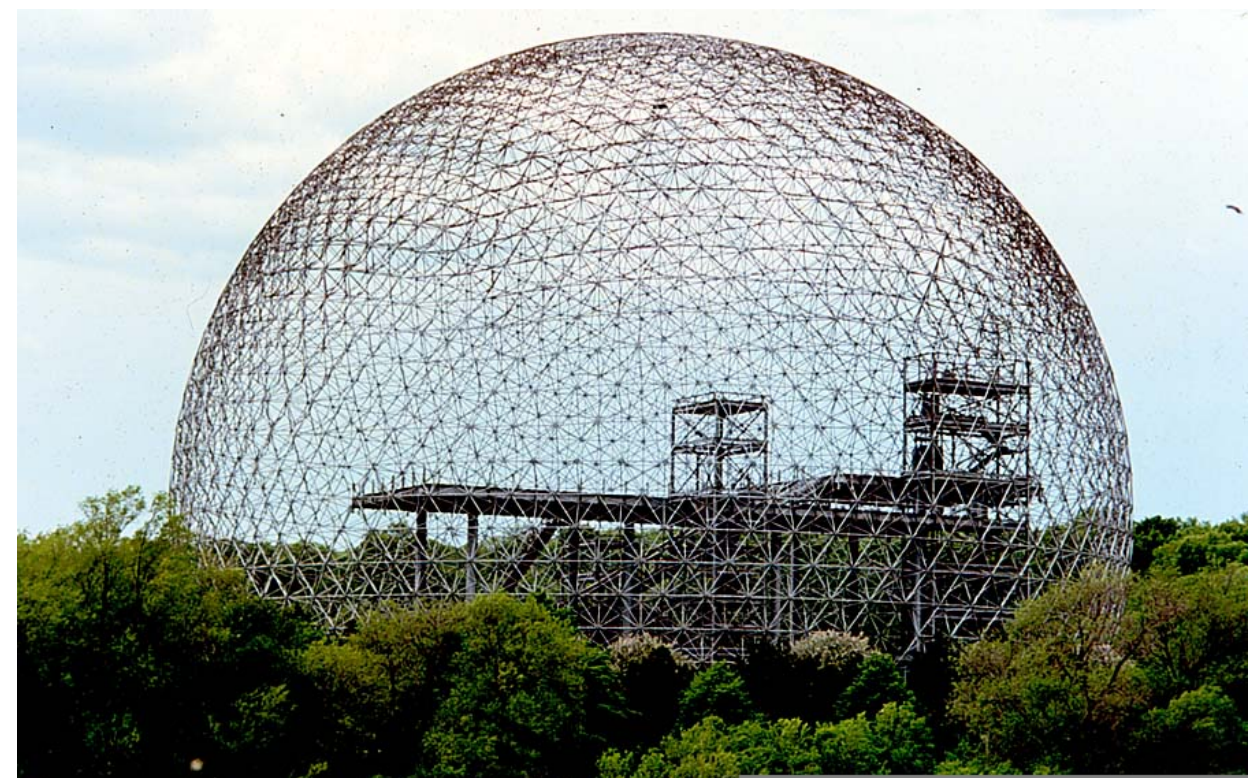

Figura 1.6: Cúpula Geodésica diseñada por el arquitecto Richard Buckminster Fuller para ser utilizada como pabellón de EEUU en la exposición universal de 1967 en Montreal (Canadá). La forma en que está construida es similar a la forma en que se disponen los átomos de carbono para dar lugar a los fullerenos, pero formando una red triangular que es dual de la hexagonal.

Kroto y Smalley llegaron a la conclusión de que la estructura de estos grupos de 60 átomos de carbono $\left(C_{60}\right)$ tenía que ser cerrada, para satisfacer todos los enlaces libres en los bordes. Sin embargo, era imposible conseguir una estructura de ese tipo únicamente con hexágonos. Recordaron entonces las cúpulas geodésicas del arquitecto norteamericano Richard Buckminster Fuller (1895-1983). Para diseñar estas estructuras, Fuller había intercalado cierto número de pentágonos entre los hexágonos. También el grupo de 60 átomos de carbono podía cerrarse si se incluían 
pentágonos en su estructura. Haciendo los cálculos de manera que se cumplieran todas las condiciones geométricas, postularon que el $C_{60}$ debía estar formado por 12 pentágonos regulares rodeados por 20 hexágonos regulares. Habían descubierto una nueva forma natural de organización del carbono.

El 14 de noviembre de 1985 se publicó el artículo en el semanario Nature. En el título del artículo aparecía la palabra que utilizaron para designar a la nueva estructura descubierta: C60: Buckminsterfullerene.

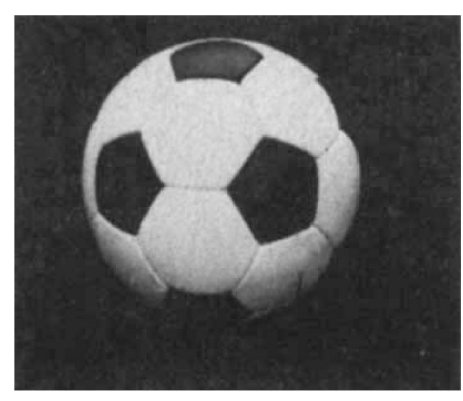

Figura 1.7: En la imagen una de las ilustraciones del artículo de Kroto y su equipo, que muestra la foto que tomaron los autores de una pelota de fútbol, muy similar a la forma que ellos postularon para el recién descubierto $C_{60}$, como puede verse en la figura.

Posteriormente siguieron apareciendo más estructuras cerradas de carbono, todas ellas compuestas por pentágonos y hexágonos. Más tarde se descubriría que la configuración que adopta cualquier fullereno debe cumplir la fórmula de Euler (1707-1783) para poliedros convexos, que relaciona el número de caras $(C)$, aristas $(A)$ y vértices $(V)$ en el mismo a través de la expresión: $C+V-A=2$. Tal y como confesarón después, las cosas hubieran sido más sencillas para los investigadores del grupo de Kroto si hubieran conocido con anterioridad este teorema. 
El artículo publicado por Kroto y su equipo no recibió la inmediata aceptación de toda la comunidad científica. Algunos investigadores que trabajaban con materiales de carbono argumentaban que nunca habían encontrado semejante agrupación. Fue necesario esperar hasta el año 1990, en que un grupo compuesto por cuatro físicos consiguió aislar cantidades macroscópicas de fullerenos (Krätschmer et al. 1990), utilizando benceno para disolver el material desprendido en el proceso de síntesis. Desde entonces se han ido encontrando cada vez más formas de fullerenos.

En 1992, Ugarte descubrió que existen otras forma de fullerenos multicapa (figura 1.8), que poseen una estructura de capas concéntricas por lo que también se los conoce como fullerene onions o buckyonions. En imágenes bajo microscopio electrónico de transmisión transmission electron microscope (TEM), los buckyonions tienen una geometría perfectamente esférica. sin embargo, estudios teóricos dicen que cuando el número de átomos es grande la geometría debería ser poliédrica. Algunos autores argumentan que es posible alcanzar la geometría esférica si los fullerenos poseen cierto número de defectos dispuestos de forma simétrica en toda la estructura (Bates y Scuseria 1998, Pérez-Garrido 2002, Pérez-Garrido et al. 2002).

Los Fullerenos presentan múltiples posibilidades de aplicación, sin embargo hoy en día todavía no han sido desarrolladas. La molécula tiene una dureza y compresibilidad formidables que la pueden hacer competir ventajosamente con el diamante. Tiene un altísimo punto de vaporización y una gran viscosidad. Además, dada su maleabilidad, puede servir de conductor o aislante eléctrico, de semiconductor o de superconductor.

Sumio Iijima, depués de un encuentro con Kroto, estuvo intentando generar fullerenos mediante una descarga de arco entre dos electrodos. Cuando examinó el material a través de un microscopio electrónico encontró los fullerenos, pero también una especie de agujas de carbono. Un estudio más detenido le permitió darse cuenta de que dichas agujas eran en realidad tubos huecos de carbono. Se trataba 

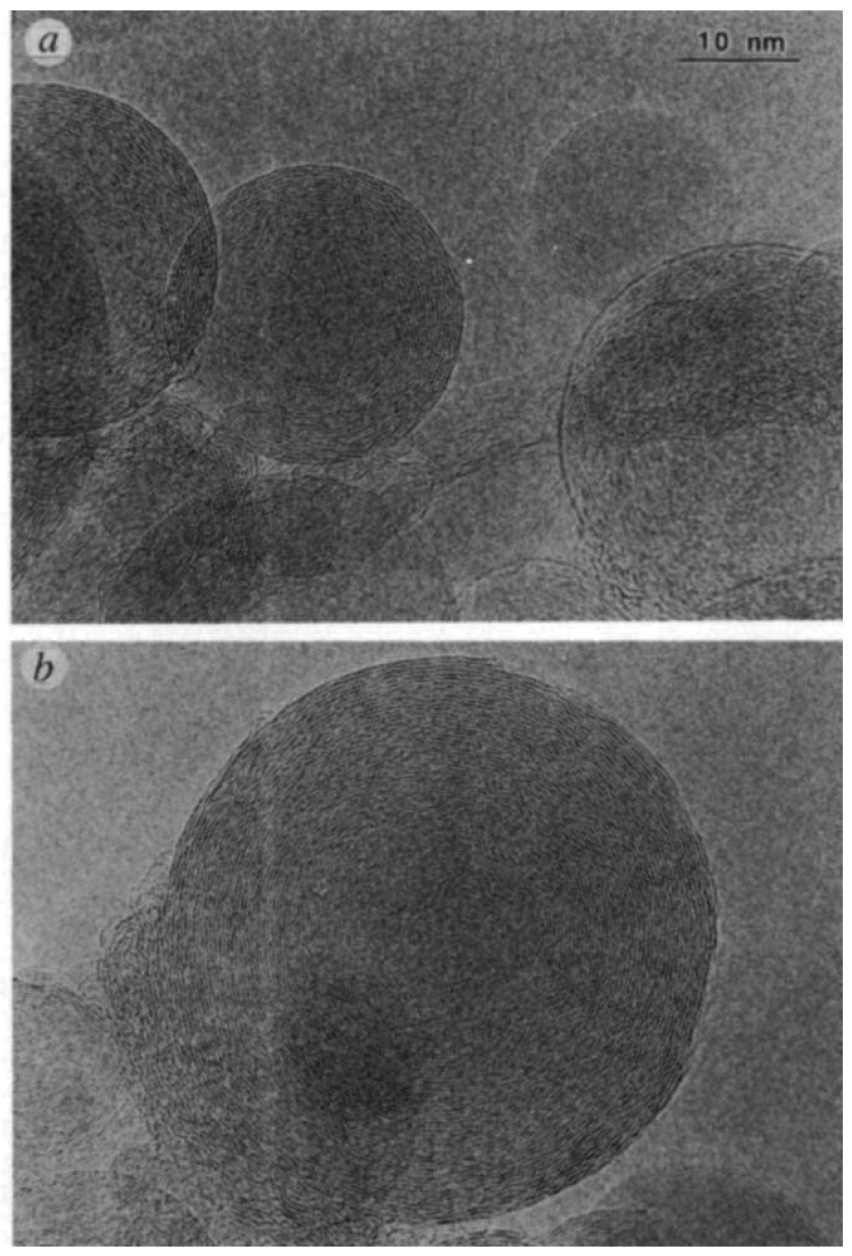

Figura 1.8: Una de las ilustraciones del artículo en el que Ugarte (Ugarte 1992) muestra la existencia de los fullerenos multicapa. Se trata de una imagen TEM en la que se aprecia la gran esfericidad de dichas estructuras. 


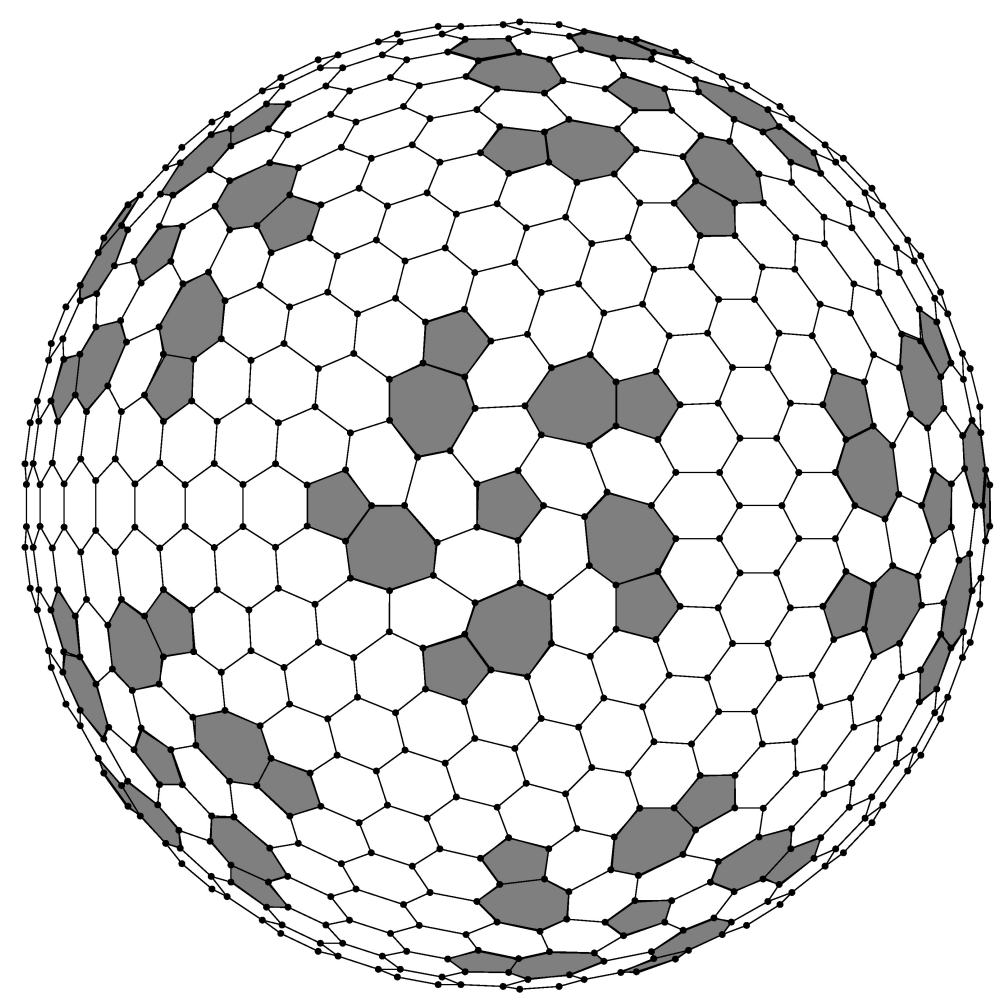

Figura 1.9: Representación esquemática de un fullereno, donde se muestran resaltados en color gris los defectos del mismo (pentágonos y heptágonos). Si el fullereno posee un número grande de átomos, la forma esférica sólo se consigue al intercalar defectos de forma simétrica a lo largo de toda la estructura. 
de una nueva clase de molécula que Smalley había predicho anteriormente como teóricamente posible. Iijima publicó su descubrimiento en 1991, momento a partir del cual comenzó la investigación en nanotubos de carbono.

\subsection{Síntesis de nanotubos de carbono}

En este apartado vamos a describir sucintamente los diferentes métodos desarrollados para la síntesis y el crecimiento de los nanotubos de carbono. El perfeccionamiento de los métodos de fabricación de CNT es hoy en día una de las líneas de desarrollo más importantes dentro de la física experimental. Es necesario sintetizar de forma controlada los diferentes tipos de CNT para lograr llevar a cabo experimentos que demuestren el gran potencial de aplicaciones tanto electrónicas como mecánicas predichas para ellos. Sin embargo, hoy en día todavía no es posible una producción de CNT totalmente controlada, ya que no podemos manejar todos los parámetros posibles para sintetizar nanotubos del tipo deseado, ni controlar el número de defectos producidos. Básicamente, los métodos de síntesis de CNT son tres, que pasamos a describir:

$\square$ Ablación mediante láser: es un procedimiento bastante efectivo para la síntesis de haces de nanotubos de una sóla capa, que se obtienen en un porcentaje en torno al 70-90\% del material empleado. Se basa en la utilización de un compuesto de grafito que sirve como blanco y cuya composición es un 98,8\% grafito y un 1,2\% una mezcla de cobalto y níquel a partes iguales que actúan como catalizadores. El blanco se introduce en un tubo de cuarzo a una temperatura en torno a $1200^{\circ} \mathrm{C}$ y se bombardea mediante un haz láser, lo que provoca la evaporación de parte del material. A continuación se utiliza una corriente de gas argón en el interior del tubo de cuarzo para depositar el material desprendido en un colector refrigerado. El dispositivo experimental puede verse en la figura 1.10 . 


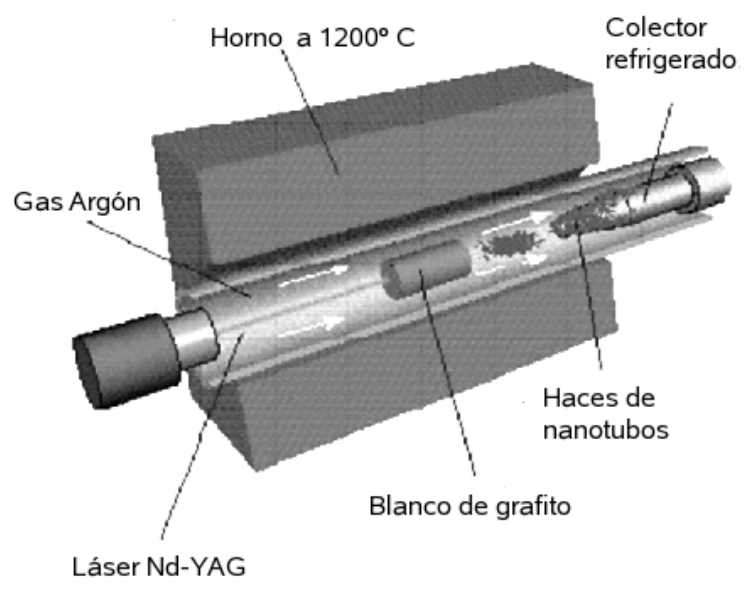

Figura 1.10: Representación esquemática del dispositivo experimental utilizado en la obtención de nanotubos mediante la técnica de ablación por láser.

El material así formado está compuesto por unas estructuras que al ser vistas a través de un microscopio electrónico se asemejan a cuerdas de 10-20 nm de diámetro y $100 \mu \mathrm{m}$ de longitud o más, por lo que reciben el nombre de nanocuerdas (nanoropes). Cada nanocuerda está formada a su vez por un racimo de CNT alineados en torno a un eje común y cuyos diáme-

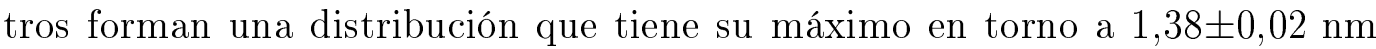
(Thess et al. 1996). Este diámetro se corresponde con un SWNT tipo $(10,10)$. En el apartado 1.4 explicaremos los tipos de nanotubos y veremos cómo calcular su diámetro. Un estudio más detallado permite observar que el $44 \%$ de los nanotubos obtenidos mediante este procedimiento son del tipo $(10,10)$. Un $20 \%$ del tipo $(9,9)$ y el resto en su mayor parte son del tipo $(12,8)$. Otros autores (Cowley et al. 1997, Cowley y Sundell 1997, Kataura et al. 1998) han obtenido diferentes distribuciones modificando la temperatura y la composición del catalizador u otros parámetros que intervienen en la formación de los nanotubos. 
$\square$ Descarga de arco: permite sintetizar tanto nanotubos de una sóla capa como nanotubos multicapa (Journet et al. 1997). Para ello se alcanzan temperaturas de más de $3000^{\circ} \mathrm{C}$ que provocan la vaporización de los átomos de carbono que pasan a estado de plasma. Dichas temperaturas se consiguen gracias a descargas de arco entre dos electrodos de carbono.

El mecanismo es el siguiente: se disponen dos electrodos de carbono en forma de barra de 5-20 mm de diámetro separados una distancia de $1 \mathrm{~mm}$, y a una diferencia de potencial de 20-25 V. Esto provoca una corriente en torno a 50120 A con una descarga de arco entre los electrodos. Dicha descarga provoca altas temperaturas que producen la vaporización de los átomos de carbono del propio electrodo. Así, el electrodo positivo (ánodo) va desgastándose a la vez que en el negativo (cátodo) van depositándose materiales de carbono que contienen los nanotubos formados. Puede verse una representación esquemática del dispositivo experimental en la figura 1.11.

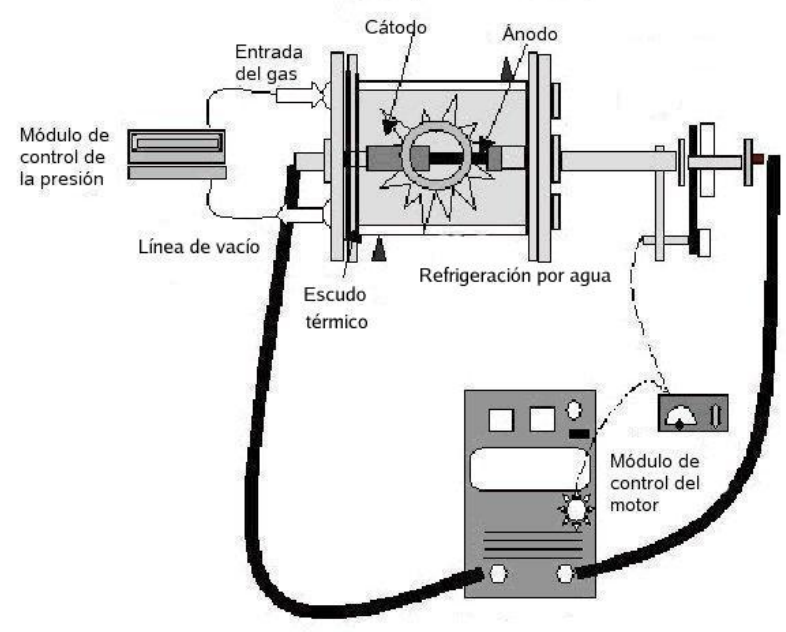

Figura 1.11: Representación esquemática del dispositivo experimental utilizado en la obtención de nanotubos mediante la técnica de descargas de arco. 
Si la temperatura se ajusta de forma correcta, no es necesario el uso de ningún tipo de catalizador para que se formen mediante este proceso los MWNT, que van depositándose en forma de racimo en el cátodo orientados de acuerdo con la dirección de la corriente (Ebbesen et al. 1993, Seraphin et al. 1993, Saito y Inagaki 1993, Ebbesen 1994). Además, se forman junto con los MWNT diversas estructuras diferentes de carbono como fullerenos y nanoestructuras en general (Ajayan y Iijima 1992, Dravid et al. 1993, Iijima 1993). Para obtener SWNT sí es necesaria la utilización de catalizadores y se suele utilizar metales de transición como hierro, cobalto o níquel o tierras raras como ytrio o gadolinio. También es necesario utilizar catalizadores para obtener racimos de SWNT. En estos casos se forman, junto con los nanotubos, nanoestructuras de otros tipos como capas de grafeno, así como agrupamientos de metales diversos, por lo que es necesario purificar el material para separar los nanotubos. Las condiciones en las que se produce la síntesis de los nanotubos, como por ejemplo la temperatura o los catalizadores utilizados, condicionan la quiralidad y el diámetro de los nanotubos que se forman mediante este procedimiento. Sin embargo, se suelen obtener nanotubos de diámetros pequeños $(<1,5 \mathrm{~nm})$.

$\square$ Depósitos de vapor, Chemical Vapor Deposition (CVD): era el procedimiento utilizado en la obtención de fibras y filamentos de carbono entre los años 1960-1970, como ya mencionamos en el apartado 1.1. Mediante este proceso se pueden formar también diversas nanoestructuras. El método de síntesis de fibras de carbono consiste en colocar en un tubo de reacción diferentes hidrocarburos $\left(\mathrm{CH}_{4}, \mathrm{C}_{6} \mathrm{H}_{6}\right.$, por ejemplo) junto con hidrógeno en estado gaseoso y catalizadores como Fe, Co y Ni (Dresselhaus et al. 1988, Oberlin et al. 1976b, Endo et al. 1976). La mezcla se lleva hasta temperaturas en torno a los $1100^{\circ} \mathrm{C}$, provocando así la vaporización de fibras de carbono. El proceso de síntesis de CNT es similar al de fibras de carbono, pero utilizando para este fin diferentes gases a baja presión (Endo et al. 1995, Endo et al. 1993). Podemos ver una 
representación esquemática del dispositivo experimental en la figura 1.12. Este método de síntesis ofrece ciertas ventajas sobre los dos anteriores, ya que se obtienen CNT con un mayor grado de pureza con un menor coste energético. Podría en un futuro utilizarse con fines comerciales si llegaran a controlarse todos los parámetros necesarios para sintetizar nanotubos con la quiralidad deseada (hoy en día esto no es posible).

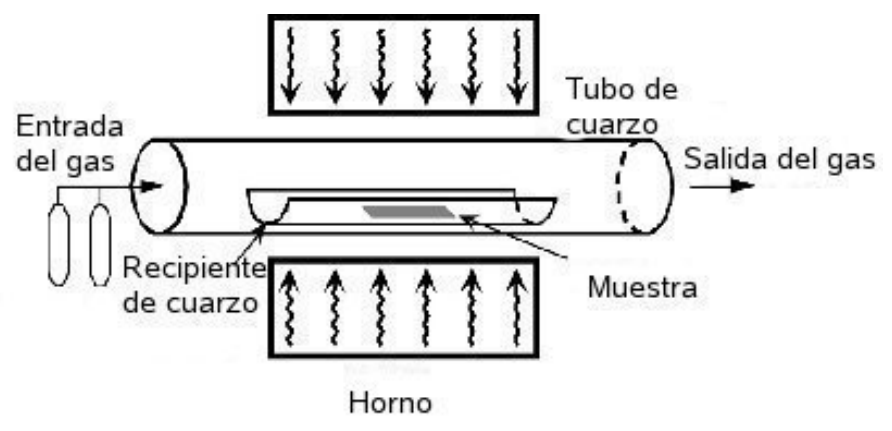

Figura 1.12: Representación esquemática del dispositivo experimental utilizado en la obtención de nanotubos mediante la técnica de depósitos de vapor.

En la figura 1.13 se muestran diversas imágenes de CNT sintetizados por los métodos descritos. Posteriormente a la síntesis de los CNT, por cualquiera de los tres métodos anteriores, es necesario llevar a cabo una serie de procesos que tienen como fin aislar y preparar los nanotubos para ser utilizados en diversos experimentos y aplicaciones. Estos procesos reciben el nombre de purificación de los nanotubos. Consisten en separar los nanotubos de los demás materiales producidos en el proceso de síntesis (nanopartículas u otras estructuras amorfas de carbono). Los más importantes son los de fase gaseosa (Ebbesen 1994, Tsang et al. 1993, Ajayan et al. 1993), los de fase líquida (Ebbesen 1997, Hiura et al. 1995) y los métodos de intercalación (Ikazaki et al. 1994). 
a)

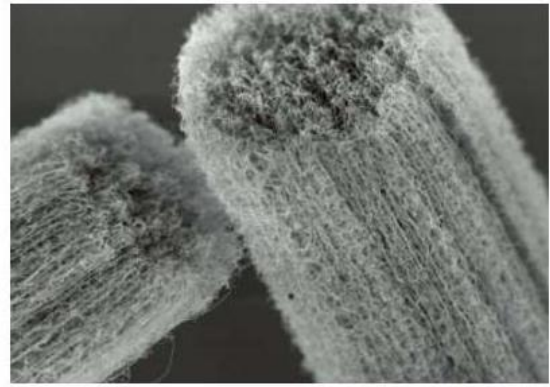

c)

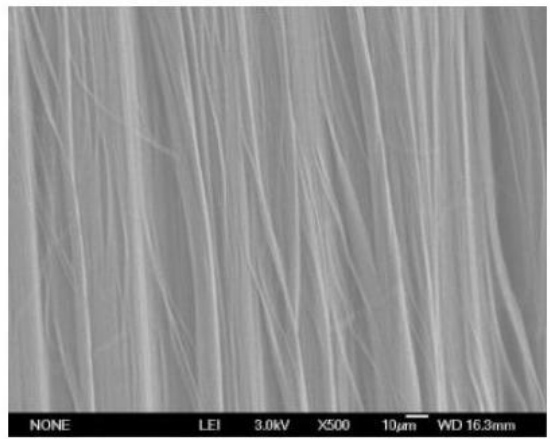

e)

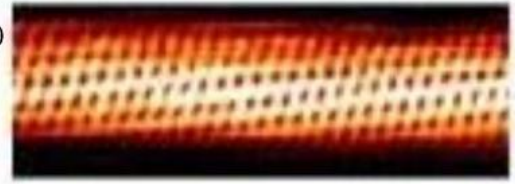

b)

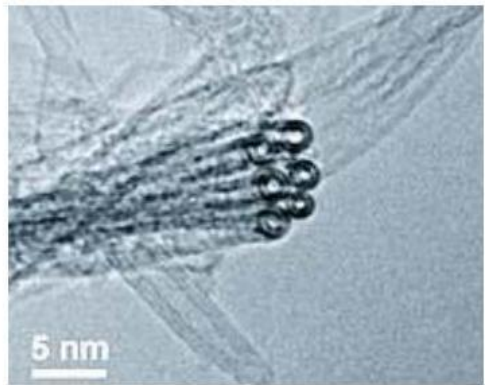

d)

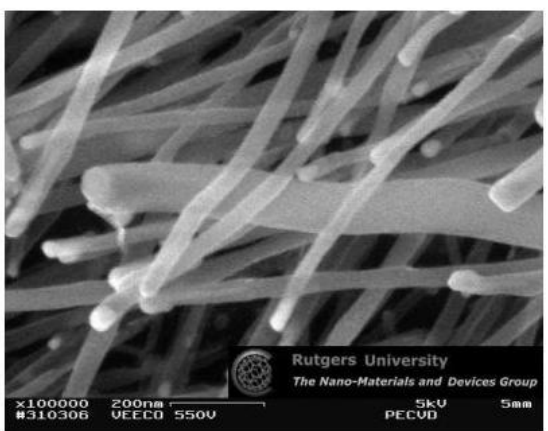

f)

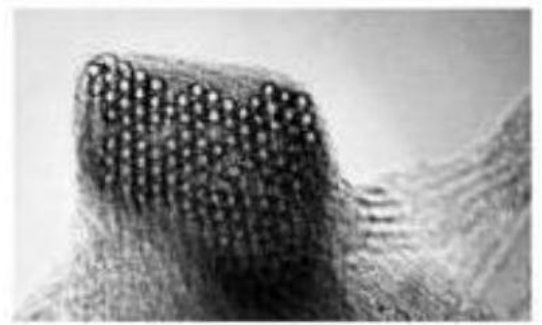

Figura 1.13: a) Crecimiento de CNT mediante el método de depósitos de vapor. Se aprecia una gran densidad de CNT de gran longitud (M. Calame, Z. Liu y C. Schönenberger. University of Base.) b) Imagen bajo microscopio electrónico de un racimo de nanotubos (Fuente: Spacemart). c) Imagen mediante microscopio electrónico de barrido de nanotubos de $1 \mathrm{~mm}$ de longitud alineados verticalmente (Firstnano. CVD Equipment Corporation). d) MWNT de diferentes grosores (Rutgers University). e) Imagen de un nanotubo quiral de 1,3 nm de diámetro generado mediante ablación por láser, obtenida mediante un microscopio de fuerza atómica (Universidad técnica de Delft. Holanda). f) Imagen TEM que muestra una nanocuerda de SWNT que han cristalizado formando una red triangular, generada mediante ablación por láser (Thess et al. (1996) Universidad de Rice). 
Pueden llevarse a cabo también procesos de manipulación de nanotubos en función de los experimentos que se deseen realizar. Entre estos procesos se encuentran, por ejemplo, la apertura del nanotubo, ya que los nanotubos sintetizados se cierran con un casquete esférico (algo así como medio fullereno) en los extremos de la parte cilíndrica (Ebbesen 1997, Dujardin et al. 1994, Ajayan y Iijima 1993). Pueden realizarse procesos de mojado (wetting) y rellenado (filling) del hueco de los nanotubos, lo cual es posible únicamente para cierto tipo de líquidos que reunen las condiciones de capilaridad necesarias (Dujardin et al. 1994, Ebbesen 1997). Para algunos experimentos es necesario que los nanotubos se alineen paralelamente unos a otros. Se han desarrollado algunos métodos de alineado de los mismos. Sin embargo, lo habitual es que el alineado se produzca directamente en el proceso de formación de los CNT. Así sucede con los obtenidos a través de las técnicas de ablación por láser y de descarga de arco. Ocasionalmente también se realiza el dopado con Boro y Nitrógeno para estudiar el comportamiento de los nanotubos tipo $p$ o tipo $n$ (Yi y Bernholc 1992), intercalado de metales para estudiar el aumento de la conductividad eléctrica (Zhou et al. 1994, Gal'pern et al. 1993) y se generan compuestos mediante capas de nanotubos de BN y C intercaladas (Blase et al. 1994, Suenaga et al. 1997).

Se han creado muchas expectativas en torno a la posibilidad de construir dispositivos electrónicos mediante CNT. Los avances experimentales en este campo muestran cómo cierto tipo de nanotubos presentan características rectificadoras, reproduciendo así el comportamiento de los diodos (Collins et al. 1997, Saito et al. 1996). Existen también trabajos sobre transistores de nanotubos (Bockrath et al. 1997, Tans et al. 1998, Yao et al. 1999). El desarrollo de dispositivos electrónicos ha potenciado las técnicas experimentales para generar nanotubos con formas determinadas que puedan tener una aplicación práctica en dichos dispositivos. Se generan nanotubos curvados (bent NT), para lo cual se utiliza la punta de un microscopio de fuerza atómica, que puede controlar la forma y posición de los nanotubos 


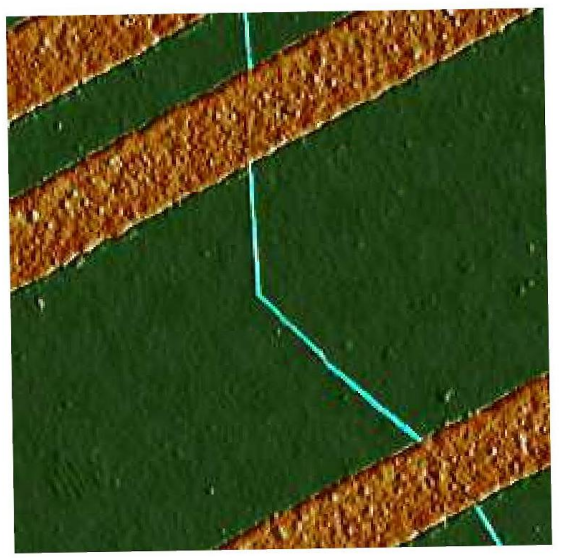

Figura 1.14: Imagen de un nanotubo con forma de codo entre dos electrodos (microscopio de fuerza atómica).

cuando éstos se encuentran sobre un sustrato de silicio. Se consigue de esta forma doblar, rectificar, trasladar, rotar e incluso cortar los nanotubos (Falvo et al. 1997, Herkel et al. 1995). También se generan codos de nanotubos (kinked NT), de los que mostramos un ejemplo en la figura 1.14. Otros trabajos se centran en provocar la unión entre nanotubos, consiguiendo de esta forma las uniones más pequeñas posibles metal-semiconductor, que producen comportamientos rectificadores en la corriente. De este modo se obtienen también nanotubos con forma de codo, al unirse nanotubos de distinta quiralidad. Para conseguirlo es necesario provocar la formación de un par de defectos (un pentágono y un heptágono) en dicha unión. Las técnicas actuales se basan en cruzar dos nanotubos diferentes, consiguiendo la unión entre ellos mediante irradiación de un haz de electrones, o bien mediante irradiación de calor (Banhart 2001, Terrones et al. 2002). También se pueden obtener estas uniones cambiando las condiciones del proceso (temperatura o catalizadores) a mitad del mismo. 
Especialmente interesantes resultan las uniones múltiples con más de dos terminales. Existen numerosos trabajos que estudian las propiedades teóricas de uniones de nanotubos en forma de $Y$ o T. Li y sus colaboradores ( $\mathrm{Li}$ et al. 1999) propusieron un método de síntesis de uniones de tipo- $Y$, que consistía en la pirólisis de acetileno utilizando en la misma plantillas de alúmina en forma de $Y$. Un equipo de investigadores (Satishkumar et al. 2000) introdujo mejoras en el método anterior, proponiendo en 2000 llevar a cabo la pirólisis de los hidrocarburos mezclándolos con tiofeno, utilizando níquel como catalizador. Consiguieron así producir una buena cantidad de nanotubos con uniones de tipo- $Y$, aunque también se obtienen con este método nanotubos con forma de codo. En cuanto a las uniones de tipo- $T$, no existe hasta el momento un método de síntesis que permita obtenerlas de forma sistemática. Existen estudios numéricos basados en modelos tight-binding que estudian las propiedades de dichas uniones de forma eficiente (Menon y Srivastava 1997, Pérez-Garrido y Urbina 2002, Menon et al. 2003). Los últimos avances experimentales hacen prever que será posible sintetizarlas en un corto plazo de tiempo. De hecho, algunos autores afirman haber encontrado uniones de tipo- $T$ cuando sintetizaban uniones de tipo- $Y$ (Deepak et al. 2006). En la figura 1.15 podemos ver imágenes TEM de ambas uniones obtenidas por los citados autores.

\subsection{Geometría y caracterización de los nanotubos de carbono}

Al comienzo de este capítulo mencionamos la importancia de la quiralidad del nanotubo como factor determinante en las propiedades del mismo (figura 1.3). Dicho factor tiene que ver con la orientación de los hexágonos formados por la red respecto

al eje del nanotubo. Para cuantificar la quiralidad se define el vector quiral $\vec{C}$, que puede verse en la figura 1.16, y cuyo significado explicamos a continuación.

Como hemos mencionado antes, podemos entender las estructura de los CNT 

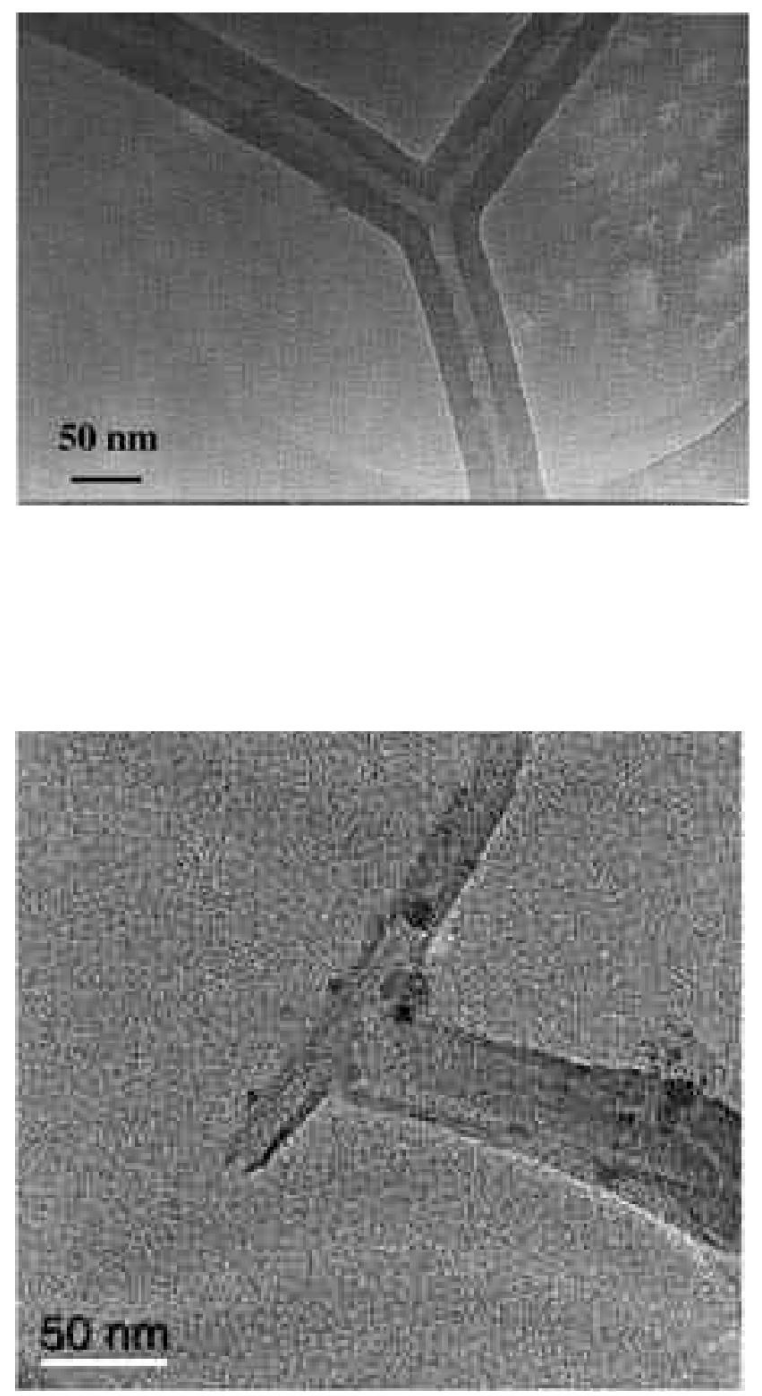

Figura 1.15: Dos imágenes TEM de uniones de tipo- $Y$ y tipo- $T$, obtenidas mediante pirólisis con tiofeno, utilizando una mezcla de níquel y dióxido de silicio como catalizadores. 
como el enrollamiento de un rectángulo de grafeno. En la figura 1.16 podemos ver el rectángulo $O B B^{\prime} O^{\prime}$, que define la celda unidad del nanotubo. Vamos a plegar ese rectángulo sobre un eje paralelo a $O B$ y de manera que identificamos $O$ con $O^{\prime}$ y $B$ con $B^{\prime}$. Las líneas $B B^{\prime}$ y $O O^{\prime}$ pasan a ser ahora círculos. El vector $O O^{\prime}$ es el que se conoce como vector quiral. Vamos a estudiar esto con un poco más de detalle.

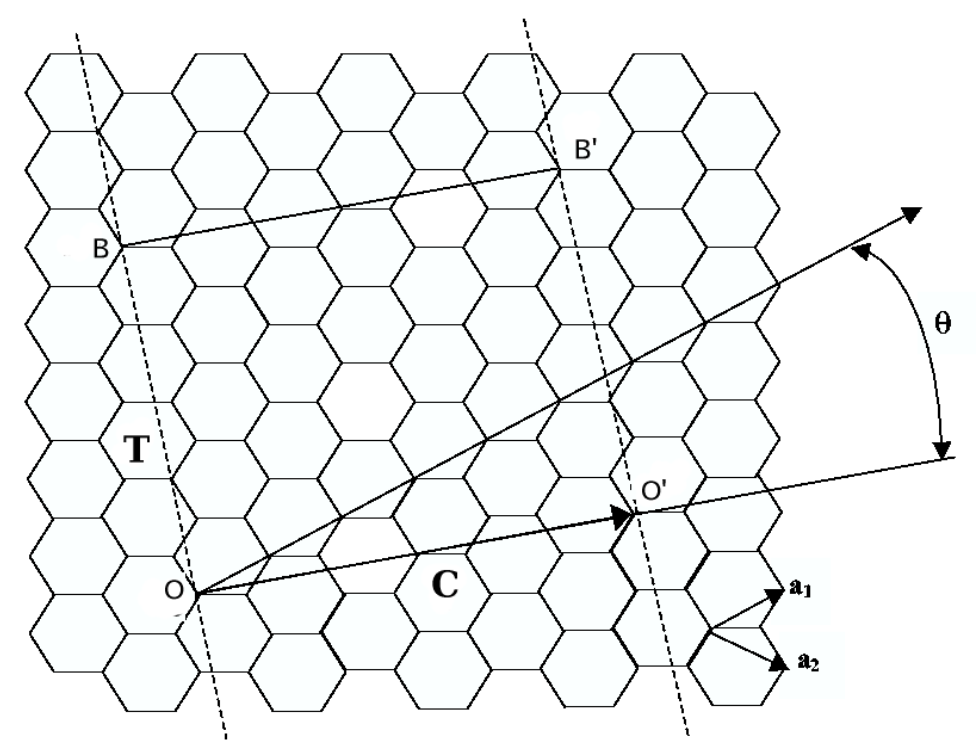

Figura 1.16: Red plana hexagonal de grafito donde se representan los vectores asociados a la construcción de un nanotubo a partir de la misma. Los puntos $O, B, B^{\prime}$ y $O^{\prime}$ definen el rectángulo a partir del cual se obtiene la celda unidad del nanotubo, resultado de enrollar dicho rectángulo llevando los puntos $O$ y $B$ a $O^{\prime}$ y $B^{\prime}$, respectivamente. Se muestran los vectores de la red $\vec{a}_{1}$ y $\vec{a}_{2}$. El vector quiral $\vec{C}$ es en este ejemplo igual a $(4,2)$ y el traslacional $\vec{T}$ tiene por valor $(4,-5)$.

Los vectores $\vec{a}_{1}$ y $\vec{a}_{2}$ representados en la figura 1.16 son los vectores base de la red hexagonal. Vienen dados por:

$$
\vec{a}_{1}=\left(\frac{\sqrt{3}}{2} a, \frac{a}{2}\right) \quad \vec{a}_{2}=\left(\frac{\sqrt{3}}{2} a,-\frac{a}{2}\right)
$$


donde $a$ es la constante de red para el grafito bidimensional. Su valor se obtiene a partir de la distancia del enlace $C-C$, igual a 1,42 $\AA$ : $a=\left|\vec{a}_{1}\right|=\left|\vec{a}_{2}\right|=1,42 \sqrt{3}=2,46 \AA$. Los vectores $\vec{a}_{1}$ y $\vec{a}_{2}$ no son ortogonales entre sí. Teniendo en cuenta que dichos vectores forman un ángulo de $60^{\circ}$ entre sí, su producto escalar cumple:

$$
\vec{a}_{1} \cdot \vec{a}_{1}=\vec{a}_{2} \cdot \vec{a}_{2}=a^{2}, \quad \vec{a}_{1} \cdot \vec{a}_{2}=a^{2} \cos 60^{\circ}=\frac{a^{2}}{2}
$$

Se define el vector quiral como el vector que va de $O$ a $O^{\prime}$ (o desde $B$ hasta $B^{\prime}$ ). A partir de los vectores $\vec{a}_{1}$ y $\vec{a}_{2}$ lo podemos expresar como:

$$
\vec{C}=n \vec{a}_{1}+m \vec{a}_{2}
$$

(donde $n$ y $m$ son cualesquiera números enteros con la condición $0 \leq|m| \leq n$ ) Podemos obtener una expresión del módulo de $\vec{C}$ a partir del producto escalar $\vec{C} \cdot \vec{C}$ teniendo en cuenta las expresiones (1.2) y (1.3):

$$
|\vec{C}|^{2}=\vec{C} \cdot \vec{C}=\left(n^{2}+m^{2}+n m\right) a^{2}
$$

Para especificar la longitud de la celda unidad del nanotubo es necesario otro vector paralelo al eje del nanotubo, cuyo extremo sea el primer átomo de la red por el que pase dicho vector. Así, se define el vector traslacional $\vec{T}$ como:

$$
\vec{T}=t_{1} \vec{a}_{1}+t_{2} \vec{a}_{2}
$$

$t_{1}$ y $t_{2}$ son números enteros. Su valor está determinado por los valores que tomen $n$ y $m$. Puesto que $\vec{C}$ y $\vec{T}$ son ortogonales entre sí su producto escalar ha de ser cero. Este hecho, junto con las ecuaciones (1.2) y (1.3), determinan el valor de $t_{1}$ y $t_{2}$ :

$$
t_{1}=\frac{2 m+n}{d_{R}}, \quad t_{2}=-\frac{2 n+m}{d_{R}},
$$


donde $d_{R}$ es el máximo común divisor de $2 m+n$ y $2 n+m$. Se puede escribir $d_{R}$ en función del máximo común divisor de $n$ y $m$ (que denotaremos como $d$ ), en la forma:

$$
d_{R}= \begin{cases}d & \text { si } n-m \text { no es múltiplo de } 3 d \\ 3 d & \text { si } n-m \text { es múltiplo de } 3 d\end{cases}
$$

expresión que se deduce de la aplicación de la ley de Euclides a $n+m$ y $n-m$. (El máximo común divisor de dos números enteros es también el máximo común divisor del resultado de la diferencia entre ambos números y el sustraendo de dicha operación).

El módulo del vector traslacional $\vec{T}$ se obtiene a partir del producto escalar $\vec{T} \cdot \vec{T}$ teniendo en cuenta las expresiones (1.2), (1.5) y (1.6):

$$
|\vec{T}|^{2}=\vec{T} \cdot \vec{T}=\frac{3 a^{2}}{d_{R}}\left(n^{2}+m^{2}+n m\right) .
$$

Con las magnitudes definidas arriba se pueden caracterizar las propiedades asociadas a cada tipo de nanotubo. Incluimos a continuación algunas de las más relevantes:

$\square$ Un tipo concreto de nanotubo queda completamente determinado por la pareja de coeficientes $n$ y $m$ asociados al vector quiral, ya que el resto de magnitudes pueden obtenerse en función de estas dos. Así, un nanotubo particular lo denotaremos como $(n, m)$. En un nanotubo tipo armchair se cumple que $m=n$ y por tanto se denota como $(n, n)$. En uno zigzag $m=0$ y por tanto se denota como $(n, 0)$.

$\square$ Puesto que el módulo del vector quiral es la longitud de la circunferencia que genera el cilindro del nanotubo, teniendo en cuenta la expresión (1.4) podemos escribir el diámetro $\left(d_{t}\right)$ del nanotubo como:

$$
d_{t}=l / \pi \quad \text { donde } \quad l=|\vec{C}|=a \sqrt{\left(n^{2}+m^{2}+n m\right)} .
$$


Ángulo de quiralidad $(\theta)$. Se define así al ángulo entre los vectores $\vec{C}$ y $\vec{a}_{1}$. Indica la orientación que toman los hexágonos de la red con respecto al eje del nanotubo. Para calcularlo utilizamos el producto escalar entre $\vec{C}$ y $\vec{a}_{1}$ teniendo en cuenta las leyes del producto escalar de $\vec{a}_{1}$ y $\vec{a}_{2}$ (ecuaciones (1.2)) y la expresión del vector quiral (1.3):

$$
\vec{C} \cdot \vec{a}_{1}=a^{2}\left(n+\frac{m}{2}\right) .
$$

El producto de los módulos de $\vec{C}$ y $\vec{a}_{1}$ resulta, teniendo en cuenta (1.4):

$$
|\vec{C}| \cdot\left|\vec{a}_{1}\right|=a^{2} \sqrt{\left(n^{2}+m^{2}+n m\right)} .
$$

Combinando las ecuaciones (1.10) y (1.11) obtenemos una expresión para el ángulo de quiralidad:

$$
\cos \theta=\frac{\vec{C} \cdot \vec{a}_{1}}{|\vec{C}| \cdot\left|\vec{a}_{1}\right|}=\frac{2 n+m}{2 \sqrt{n^{2}+m^{2}+n m}} .
$$

El ángulo de quiralidad toma valores entre $0^{\circ}$ y $30^{\circ}$. Para valores intermedios de $\theta$ la geometría es quiral. Los valores extremos, $0^{\circ}$ y $30^{\circ}$, se corresponden con un nanotubo tipo zigzag y tipo armchair, respectivamente. Podemos comprobarlo en ambos casos sustituyendo en la ecuación (1.12). Así:

$$
\begin{gathered}
\text { Armchair : } \mathrm{n}=\mathrm{m}, \quad \cos \theta=\frac{3 \mathrm{n}}{2 \sqrt{3 \mathrm{n}^{2}}}=\frac{\sqrt{3}}{2} \Rightarrow \theta=30^{\circ} . \\
\text { Zigzag : } \mathrm{n}>\mathrm{m}=0, \quad \cos \theta=\frac{2 \mathrm{n}}{2 \sqrt{\mathrm{n}^{2}}}=1 \quad \Rightarrow \quad \theta=0^{\circ} .
\end{gathered}
$$

Longitud de la celda unidad. El módulo del vector traslacional nos da la longitud de la celda unidad. Teniendo en cuenta las expresiones (1.8) y (1.9) podemos expresar su valor en función del módulo del vector quiral $(l)$ :

$$
|\vec{T}|=\frac{\sqrt{3} l}{d_{R}} .
$$


$\square$ Número de hexágonos en cada celda unidad $(N)$. Se calcula dividiendo el área de toda la celda unidad entre el área de uno de sus hexágonos. Dichas áreas se calculan mediante los productos vectoriales de los vectores representativos de las mismas. Para la celda unidad tomamos $\vec{C} \times \vec{T}$. El área de un hexágono coincide con el área del rombo generado por $\vec{a}_{1}$ y $\vec{a}_{2}$, por lo que podemos calcularla mediante el producto $\vec{a}_{1} \times \vec{a}_{2}$. Para calcular este último tendremos en cuenta (1.1):

$$
\vec{a}_{1} \times \vec{a}_{1}=\vec{a}_{2} \times \vec{a}_{2}=0, \quad \vec{a}_{1} \times \vec{a}_{2}=\frac{\sqrt{3} a^{2}}{2} .
$$

El producto vectorial entre $\vec{C}$ y $\vec{T}$ lo calculamos teniendo en cuenta los productos vectoriales de $\vec{a}_{1}$ y $\vec{a}_{2}$, junto con las expresiones (1.3), (1.5) y (1.6):

$$
\vec{C} \times \vec{T}=-\frac{\sqrt{3} a^{2}}{d_{R}}\left(n^{2}+m^{2}+n m\right) .
$$

Así obtenemos:

$$
N=\frac{|\vec{C} \times \vec{T}|}{\left|\vec{a}_{1} \times \vec{a}_{2}\right|}=\frac{2\left(m^{2}+n^{2}+n m\right)}{d_{R}} .
$$

$\square$ Número de átomos de carbono en cada celda unidad $\left(N_{a}\right)$. Podemos considerar que por cada hexágono hay dos átomos de carbono, ya que todos los átomos están compartidos por tres hexágonos. Así, el número de átomos de carbono en la celda unidad es el doble que el número de hexágonos en la misma, es decir:

$$
N_{a}=2 N=\frac{4\left(m^{2}+n^{2}+n m\right)}{d_{R}} .
$$

\subsection{Estructura electrónica}

La estructura electrónica de los nanotubos de carbono se obtiene de manera sencilla a partir de la correspondiente para el grafito plano bidimensional. Para ello es necesario imponer condiciones de frontera periódicas a lo largo de la dirección 
indicada por el vector quiral. Así, se obtiene un espectro energético continuo a lo largo de la dirección del vector traslacional (siempre y cuando consideremos el nanotubo de longitud infinita), pero cuantizado a lo largo de la dirección del vector quiral.

No entraremos en detalles matemáticos sobre la obtención de la estructura de bandas en el grafito bidimensional, lo cual no es objeto de esta memoria, pero sí hemos de recoger los resultados y aproximaciones considerados en su cálculo, ya que partiremos de los mismos para obtener la estructura de bandas de los SWNT. Recogemos a continuación de forma resumida el proceso llevado a cabo para la obtención de la estructura de bandas en las láminas de grafito bidimensionales, así como los resultados más relevantes:

Consideraremos una única capa de grafito plana bidimensional. Si tuviéramos varias capas de grafito formando una estructura 3D, la interacción entre las diferentes capas del grafito puede despreciarse, ya que la separación entre capas $(3,35 \AA)$ es mucho mayor que la distancia entre los vecinos próximos dentro de una misma capa $(1,42 \AA)$. Así pues, podemos realizar el cálculo de la estructura de bandas de una sóla capa de grafito (en 2D) sin considerar las posibles interacciones con otras capas.

Diremos que dos átomos son vecinos próximos si están unidos mediante un enlace covalente. En nuestra aproximación vamos a considerar un único orbital $\pi$ por átomo, despreciando la interacción entre segundos vecinos, esto es, entre dos átomos que, estando enlazados a un tercero, no están enlazados entre sí. La energía de solapamiento entre vecinos próximos se toma para el grafito en torno a $t=-3 \mathrm{eV}$, valor que disminuye ligeramente en el nanotubo, para el cual se toma el valor $t=-2,66 \mathrm{eV}$ (Blase et al. 1994). La ventaja de esta aproximación es su sencillez de cálculo. Puede comprobarse que utilizando este modelo las propiedades generales de transporte coinciden con aquellas 
obtenidas por otras aproximaciones en las que se tienen en cuenta más orbitales por átomo o hibridación de orbitales (Orlikowski et al. 2001).

$\checkmark$ Se realiza el cálculo de la estructura de bandas a través de un modelo tightbinding. Dejamos los detalles del modelo para el capítulo siguiente, dedicado al método de cálculo. Dicho modelo conduce a la ecuación secular:

$$
\operatorname{det}[\hat{H}-E \hat{S}]=0
$$

donde $\hat{H}$ es el hamiltoniano del sistema y $\hat{S}$ la matriz de solapamiento. Para la obtención de los autovalores y autovectores de la ecuación anterior es necesario determinar la región de Brillouin y la red recíproca, seleccionando los puntos de simetría en dicha región.

$\square$ La región de Brillouin se representa en la figura 1.17 b), como la zona sombreada en azul. El origen de la red recíproca es el punto $\Gamma$, que junto con $K$ y $M$ son los tres puntos de simetría. Los vectores de la red recíproca se representan también en esta figura como $\vec{b}_{1}$ y $\overrightarrow{b_{2}}$. Son perpendiculares a $\overrightarrow{a_{2}} \mathrm{y}$ $\overrightarrow{a_{1}}$, respectivamente, y nos dan una constante de red recíproca igual a $\frac{4 \pi}{\sqrt{3} a}$. El valor de $\vec{b}_{1}$ y $\vec{b}_{2}$ se puede obtener mediante los productos escalares de $\vec{a}_{j}$ y $\vec{b}_{i}$, que para toda red recíproca cumplen que $\vec{b}_{i} \cdot \vec{a}_{j}=2 \pi \delta_{i j}$. Así:

$$
\vec{b}_{1}=\left(\frac{2 \pi}{\sqrt{3} a}, \frac{2 \pi}{a}\right), \quad \vec{b}_{2}=\left(\frac{2 \pi}{\sqrt{3} a},-\frac{2 \pi}{a}\right)
$$

$\square$ Finalmente, teniendo en cuenta todas las consideraciones anteriores se obtiene la relación de dispersión para el grafito bidimensional como:

$$
E_{g 2 D}(\vec{k})=\frac{\epsilon_{2 p} \pm t \omega(\vec{k})}{1 \pm s \omega(\vec{k})}
$$

donde $\epsilon_{2 p}$ es la energía de sitio y s es la integral de solapamiento entre átomos vecinos. Se obtienen dos bandas de energía. El signo positivo en numerador 
a)

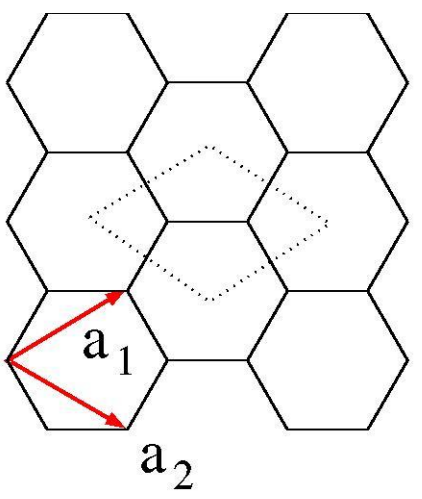

b)

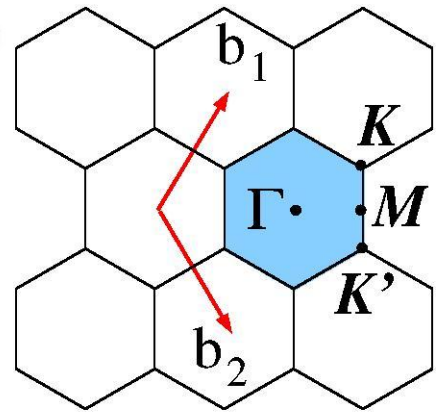

Figura 1.17: En la figura a) se representan ocho hexágonos de grafeno de la red real. La celda unidad del grafeno se corresponde con la región delimitada por el rombo punteado, dado por los vectores de la red. En la figura b) se representa la red recíproca del grafeno. La región sobreada es la primera zona de Brillouin. Se representan también en esta figura los vectores de la red recíproca y los puntos de simetría.

y denominador de la expresión anterior representa la banda de energía de los estados que denotaremos $\pi$ (enlazantes), mientras que los signos negativos representan los estados que denotaremos como $\pi^{*}$ (antienlazantes). La función $\omega(\vec{k})$ viene dada por:

$$
\omega(\vec{k})=\sqrt{1+4 \cos \left(\frac{\sqrt{3} k_{x} a}{2}\right) \cos \left(\frac{k_{y} a}{2}\right)+4 \cos ^{2}\left(\frac{k_{y} a}{2}\right)}
$$

donde se ha descompuesto el vector $\vec{k}$ según el sistema de coordenadas $x$-y de la red real en $k_{x}$ y $k_{y}$.

En la figura 1.18 podemos ver las relaciones de dispersión (1.22) para el grafito bidimensional, a lo largo de la primera zona de Brillouin. En dicha representación se han utilizado los valores numéricos $\epsilon_{2 p}=0, t=-2,9 \mathrm{eV}$ y $s=0,129$ (Saito et al. 2000). La parte superior de la figura muestra la región $\pi^{*}$, mientras que la parte inferior muestra la región $\pi$. Ambas bandas están 


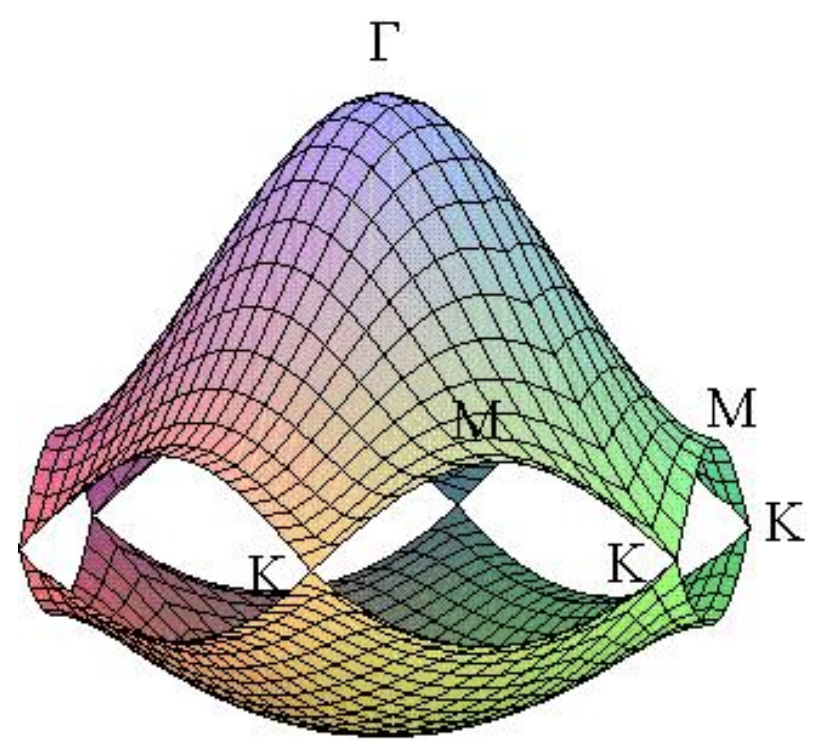

Figura 1.18: Relaciones de dispersión para el grafito bidimensional. Se representa la primera región de Brillouin.

doblemente degeneradas en los puntos $K$, que determinan la energía de Fermi. En general, las bandas $\pi$ y $\pi^{*}$ no tienen por qué ser simétricas. Sin embargo, ambas bandas son simétricas en torno a $\epsilon_{2 p}$ si se toma la integral de solapamiento igual a cero $(s=0)$, según se deduce de la ecuación (1.22). Esta es la aproximación de Slater-Koster, que utilizaremos en lo sucesivo. Teniendo en cuenta dicha aproximación, las relaciones de dispersión se escriben como:

$$
E_{g 2 D}\left(k_{x}, k_{y}\right)= \pm t\left[1+4 \cos \left(\frac{\sqrt{3} k_{x} a}{2}\right) \cos \left(\frac{k_{y} a}{2}\right)+4 \cos ^{2}\left(\frac{k_{y} a}{2}\right)\right]^{1 / 2}
$$

A partir de la ecuacion (1.24) obtendremos la relación equivalente para el SWNT imponiendo las condiciones específicas del nanotubo considerado. En primer lugar, remplazamos los vectores de la red del grafito $\vec{a}_{1}$ y $\vec{a}_{2}$ por los vectores que determi- 
nan el nanotubo, $\vec{C}$ y $\vec{T}$. Para calcular los vectores de la red recíproca, $\vec{K}_{1}$ y $\vec{K}_{2}$, realizamos los productos escalares: $\vec{C} \cdot \vec{K}_{2}=0, \vec{C} \cdot \vec{K}_{1}=2 \pi, \vec{T} \cdot \vec{K}_{1}=0$ y $\vec{T} \cdot \vec{K}_{2}=2 \pi$. Se obtienen así las componentes de $\vec{K}_{1}$ y $\vec{K}_{2}$ en función de $\vec{b}_{1}$ y $\vec{b}_{2}$ :

$$
\vec{K}_{1}=\frac{-t_{2} \vec{b}_{1}+t_{1} \vec{b}_{2}}{N}, \quad \vec{K}_{2}=\frac{m \vec{b}_{1}-n \vec{b}_{2}}{N},
$$

donde $N$ es el número de hexágonos de la celda unidad (ecuación 1.18).

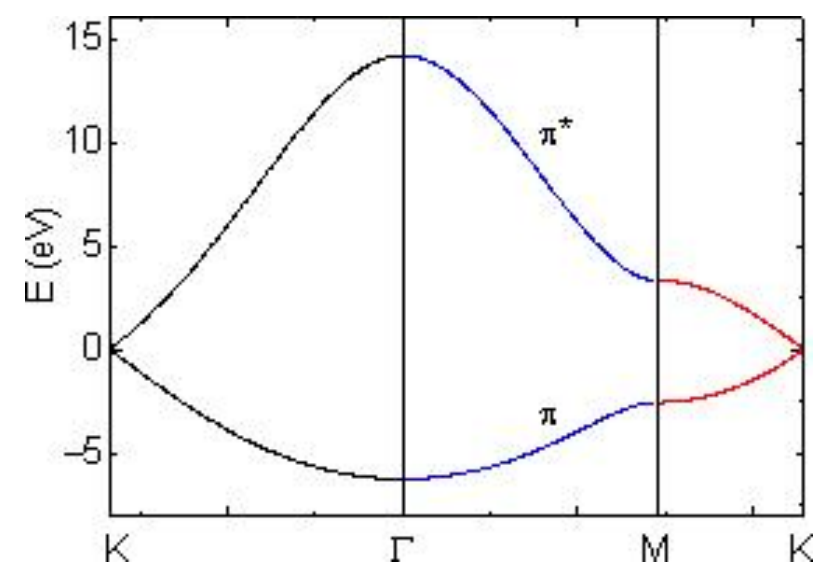

Figura 1.19: Sección transversal de la relación de dispersión del grafito, que muestra una de las posibles bandas de energía para un nanotubo de carbono.

Puesto que el espectro ha de ser continuo a lo largo de la dirección del eje del nanotubo, dada por $\vec{T}$, y discreto a lo largo de la circunferencia del nanotubo, dada por $\vec{C}$, las bandas de energía han de ser un conjunto de relaciones de dispersión unidimensionales que serán secciones transversales de las del grafito bidimensional. Así, podemos escribir la relación de dispersión para el nanotubo en 1D como:

$$
E_{\mu}=E_{g 2 D}\left(k \frac{\vec{K}_{2}}{\left|\vec{K}_{2}\right|}+\mu \vec{K}_{1}\right)
$$


donde $\mu=0, \ldots, N-1 \mathrm{y}-\frac{\pi}{T}<k<\frac{\pi}{T}$. Las $N$ parejas de curvas de dispersión dadas por la ecuación anterior se corresponden con las citadas secciones transversales de la superficie bidimensional formada por las relaciones de dispersión del grafito. En la figura 1.19 se muestra una de las secciones transversales de la figura 1.18.

Si para un nanotubo $(n, m)$ cualquiera las líneas de corte pasan por el punto $K$ de la región de Brillouin, no existirá un gap de energía entre las dos bandas y por tanto el nanotubo será metálico, como en la figura 1.19. Por el contrario, si las líneas de corte no pasan por $K$, existirá un gap finito de energía entre las bandas de conducción y valencia y el nanotubo será un semiconductor.

a)

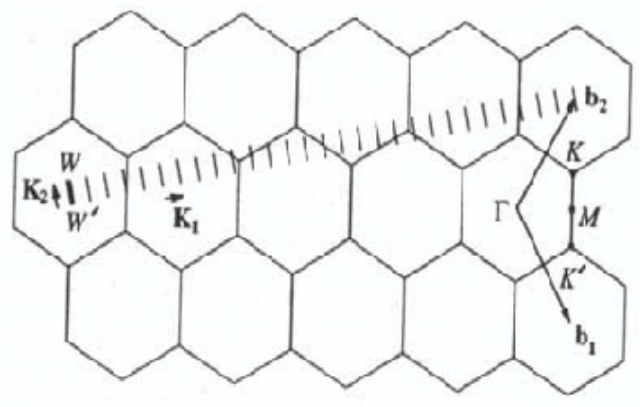

b)

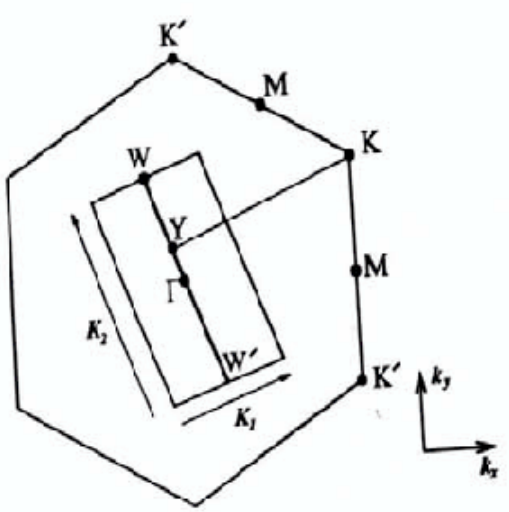

Figura 1.20: a) Primera región de Brillouin de un CNT $(4,2)$. En la figura b) puede verse que la condición para que un nanotubo sea metálico es que la distancia desde el punto $Y$ al $K$ sea un múltiplo entero de la longitud del vector $\vec{K}_{1}$.

En la figura 1.20 a) se muestra la primera región de Brillouin para un nanotubo $(4,2)$, que queda determinada por los vectores $\vec{K}_{1}$ y $\vec{K}_{2}$. Podemos obtener una condición para deducir el carácter metálico o semiconductor de un nanotubo analizando la relación entre los vectores $\overrightarrow{Y K}$ y $\vec{K}_{1}$ de la figura 1.20 b): 


$$
\overrightarrow{Y K}=\frac{2 n+m}{3} \vec{K}_{1} .
$$

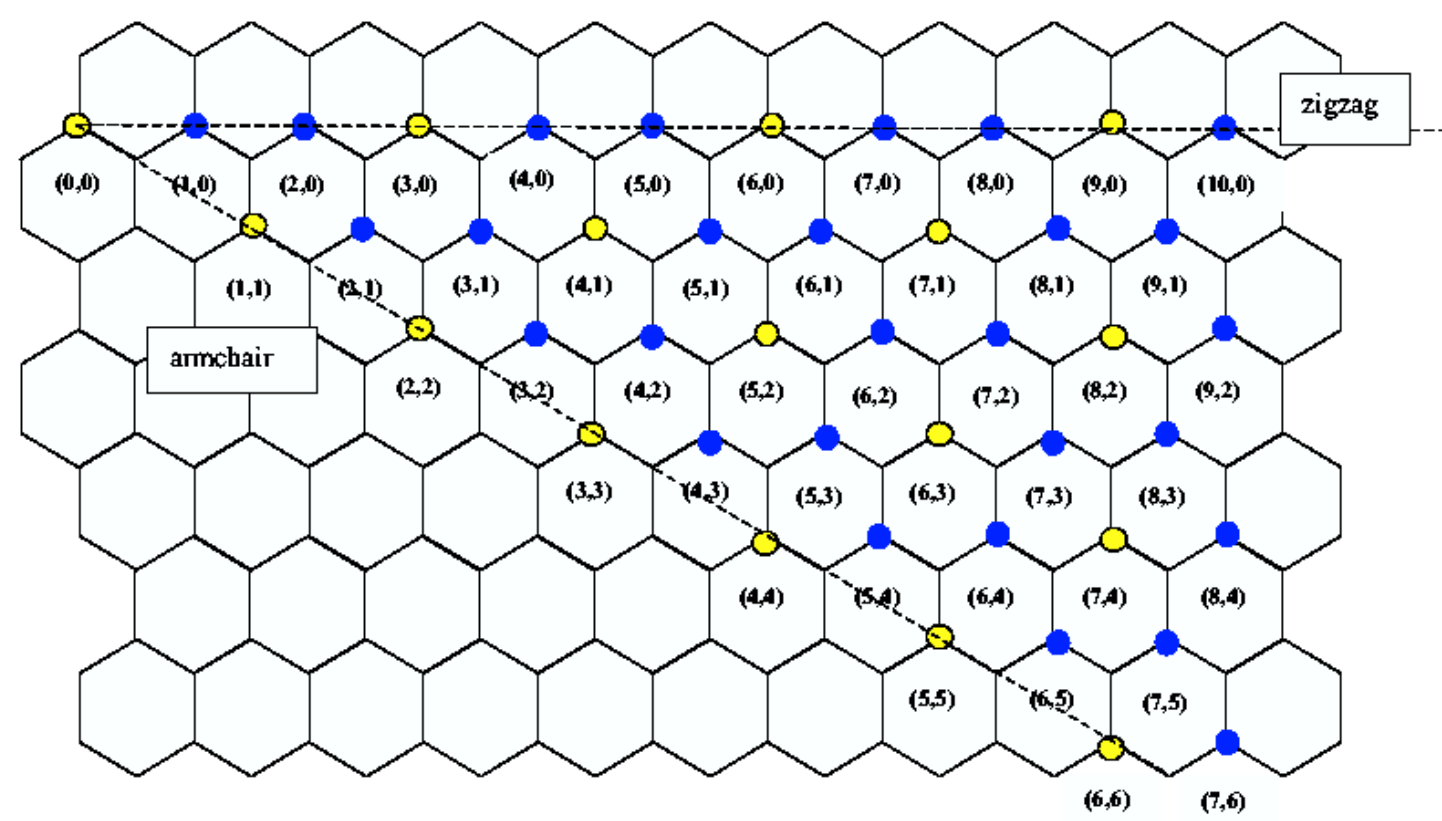

Figura 1.21: En la figura se representan mediante círculos amarillos los nanotubos con carácter metálico. Los círculos de color azul representan nanotubos con carácter semiconductor.

Para que un nanotubo sea metálico la razón entre la longitud del vector $\overrightarrow{Y K}$ y $\vec{K}_{1}$ ha de ser un número entero, como puede deducirse de la figura $1.20 \mathrm{~b}$ ). Así pues, la condición necesaria y suficiente para que un nanotubo presente carácter metálico es que $2 n+m$, o lo que es lo mismo, $n-m$ sea múltiplo de 3. En la figura 1.21 se representan los nanotubos que son metálicos y semiconductores mediante círculos amarillos y azules, respectivamente. En particular, todos los nanotubos tipo armchair $(n, n)$ son metálicos, mientras que para que un nanotubo zigzag $(n, 0)$ sea 
metálico $n$ debe ser múltiplo de 3 .

Finalmente, podemos obtener expresiones analíticas a partir de la ecuación (1.26) para casos de geometrías sencillas, como lo son las de los nanotubos tipo armchair y zigzag. Escribiendo las condiciones de frontera adecuadas en cada caso obtenemos, para los nanotubos tipo armchair y zigzag las ecuaciones que nos proporcionan la estructura de bandas de ambos tipos de nanotubos.

$\square$ Nanotubo tipo armchair:

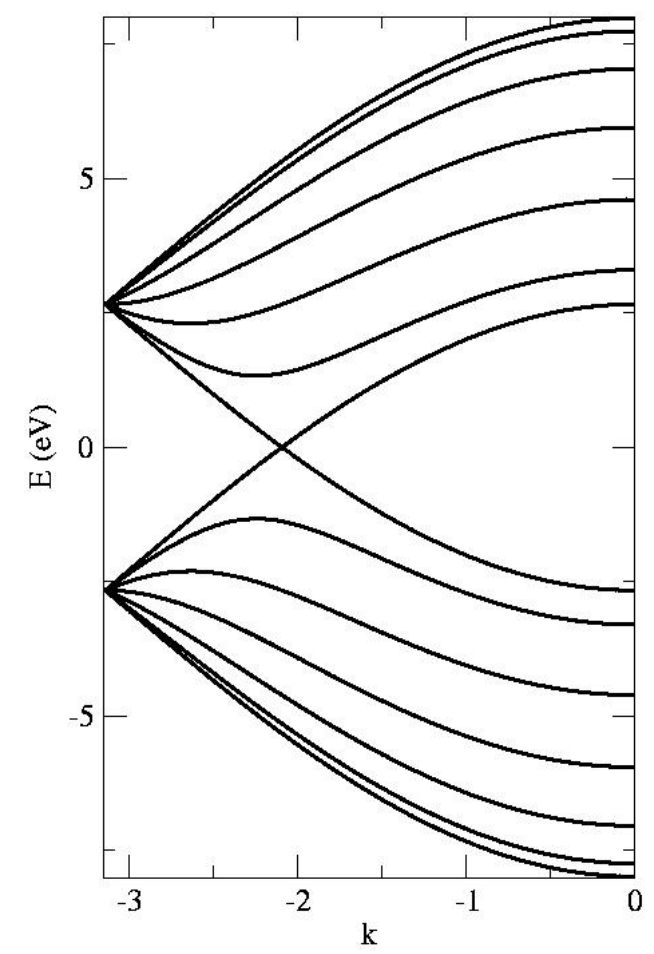

Figura 1.22: Estructura de bandas de un nanotubo $(6,6)$. El carácter conductor de dicho nanotubo se pone de manifiesto en el corte de las bandas de conducción y de valencia para la energía de Fermi. 
Las condiciones de frontera para obtener los autovalores de la energía definen los vectores permitidos a lo largo de la circunferencia del nanotubo. En un nanotubo tipo armchair dichos vectores son aquellos que cumplen la siguiente condición:

$$
n \sqrt{3} k_{x, q} a=2 \pi q \quad(q=1, \cdots, 2 n) .
$$

Sustituyendo la expresión (1.28) en (1.24) se obtienen las relaciones de dispersión para un nanotubo tipo armchair $(n, n)$ :

$$
E_{q}^{a}= \pm t\left[1 \pm 4 \cos \left(\frac{q \pi}{n}\right) \cos \left(\frac{k a}{2}\right)+4 \cos ^{2}\left(\frac{k a}{2}\right)\right]^{1 / 2} .
$$

donde $-\pi<k a<\pi$ y $q=1, \ldots, 2 n$.

En la expresión (1.29) $k$ representa un parámetro vectorial unidimensional en la dirección de $\vec{K}_{2}$. En la figura 1.22 se muestran las bandas de energía obtenidas mediante la citada expresión, para un nanotubo $(6,6)$. Puede verse en la figura que tanto la banda de conducción como la de valencia presentan 7 relaciones de dispersión. 5 de estas relaciones son doblemente degeneradas, por lo que en realidad tenemos 12 niveles en cada una de las bandas de energía. Todos los nanotubos tipo armchair son conductores, 0 es múltiplo de 3 , lo que significa que no existe un gap de energía entre la banda de conducción y de valencia. Como comprobamos en la figura 1.22, dichas bandas se cortan para una energía igual a la de Fermi.

$\square$ Nanotubo tipo zigzag:

En un nanotubo tipo zigzag los vectores permitidos a lo largo de la circunferencia del nanotubo cumplen la siguiente condición: 

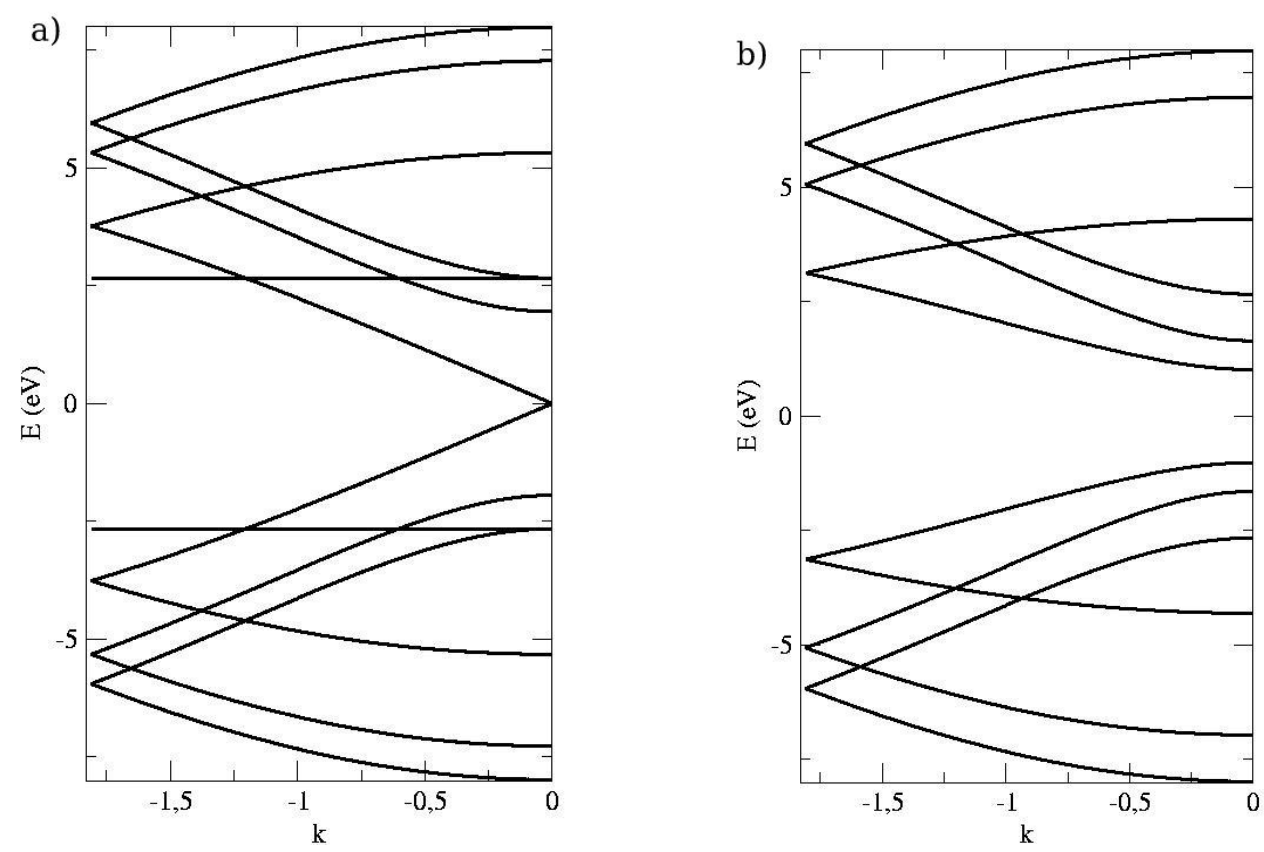

Figura 1.23: a) Estructura de bandas de un nanotubo $(6,0)$. Las bandas de conducción y valencia se cortan para la energía de Fermi. b) Estructura de bandas de un nanotubo $(5,0)$. En este caso existe un gap entre las bandas de conducción y valencia. 


$$
n k_{y, q} a=2 \pi q \quad(q=1, \cdots, 2 n) .
$$

Sustituyendo la expresión (1.30) en (1.24) se obtienen las relaciones de dispersión para un nanotubo tipo zigzag $(n, 0)$ :

$$
E_{q}^{z}= \pm t\left[1 \pm 4 \cos \left(\frac{\sqrt{3} k a}{2}\right) \cos \left(\frac{q \pi}{m}\right)+4 \cos ^{2}\left(\frac{q \pi}{n}\right)\right]^{1 / 2}
$$

donde $-\frac{\pi}{\sqrt{3}}<k a<\frac{\pi}{\sqrt{3}}$ y $q=1, \ldots, 2 n$.

Un nanotubo tipo zigzag puede presentar carácter metálico o semiconductor dependiendo del valor de $n$. Si $n$ es múltiplo de 3 el nanotubo es metálico, mientras que si $n$ no es múltiplo de 3 el nanotubo es semiconductor. En la figura 1.23 se representan ambos casos. La figura a) representa la estructura de bandas de un nanotubo $(6,0)$. Para este nanotubo nos encontramos nuevamente con 7 relaciones de dispersión en cada banda de energía, 5 de las cuales son doblemente degeneradas, por lo que existen 12 niveles por cada banda. Para la energía de Fermi ambas bandas se cortan por lo que el nanotubo representado tiene carácter metálico. En la figura b) se representa la estructura de bandas para un nanotubo $(5,0)$. En este caso existen 10 niveles de energía por cada banda. Se aprecia un pequeño gap entre las bandas de conducción y valencia, lo que indica que en este caso el nanotubo representado posee carácter semiconductor.

\subsection{Líneas de investigación}

El estudio tanto teórico como experimental de las propiedades de los CNT ha atraido la atención de numerosos investigadores desde el momento en que fueron descubiertos en 1991 por Iijima. Como ya hemos mencionado anteriormente, este interés es debido en gran parte a las características electrónicas de los CNT, que 
hacen de ellos una fuente de resultados experimentales y teóricos que puede tener una gran repercusión en física de semiconductores. Así mismo, poseen excelentes propiedades mecánicas debido a que su estructura posee una gran fuerza y rigidez, a la vez que su densidad es muy baja. En los siguientes subapartados desarrollamos un poco el estudio de las propiedades electrónicas y mecánicas.

\subsubsection{Propiedades electrónicas}

En cuanto a las propiedades electrónicas de transporte en nanotubos, podemos resumir las investigaciones realizadas clasificándolas en tres grandes grupos:

$\square$ Propiedades de los CNT con defectos o deformaciones. Se han estudiado ampliamente los efectos de la presencia de defectos topológicos como pentágonos, heptágonos y huecos en los CNT. En particular, se ha analizado su influencia en la densidad de estados, la conductancia y la localización de estados debido a dichos defectos (Kostyrko et al. 1999a, Kostyrko et al. 1999b, Choi et al. 2000, Song et al. 2002). Del mismo modo se han estudiado tambión los efectos de las deformaciones estructurales, como deformaciones radiales, en las propiedades de conducción (Sim et al. 2001, Gülseren et al. 2002).

$\neg$ Dispositivos electrónicos. Existen numerosos trabajos que se centran en obtener y caracterizar las propiedades de conducción entre uniones de diferentes nanotubos. Estas uniones pueden poseer dos, tres o hasta cuatro terminales (Chico et al. 1996, Menon y Srivastava 1997, Tamura y Tsukada 1999, Andriotis et al. 2002, Grimm et al. 2003, del Valle et al. 2005). Algunas de estas uniones han generado grandes expectativas en torno a la utilización de las mismas como dispositivos electrónicos. Hasta el momento se han observado características rectificadoras que reproducen en cierta parte el comportamiento de los diodos en uniones metal-semiconductor (Collins et al. 1997, 
Saito et al. 1996, Farajian et al. 2004). Así mismo, algunos autores han encontrado comportamientos similares a los de los transistores (Tans et al. 1998, Bockrath et al. 1997, Yao et al. 1999).

그os fenómenos cuánticos. El estudio sistemático de las propiedades de conducción entre uniones de nanotubos ha reportado también la observación de diversos fenómenos de interés como transporte cuántico por efecto túnel, interferencias cuánticas o puntos cuánticos. (Chico et al. 1996, Orlikowski et al. 2001, Sim et al. 2001, Rocha et al. 2002, Chico y Jaskolski 2004). Mención especial merece el fenómeno de los puntos cuánticos, al que dedicamos el siguiente apartado.

\subsubsection{Propiedades mecánicas}

Una gran parte de las investigaciones se centran en cuantificar las constantes elásticas de las diferentes geometrías de nanotubos. Se han llegado a medir valores del módulo de Young del orden de $1 \mathrm{TPa}$, y una tensión de ruptura del orden de 150 GPa para MWNT (Demczyk et al. 2002), medidas para un nanotubo de diámetro igual a 12,5 nm. Diferentes cálculos teóricos conducen a resultados del mismo orden (Jin y Yuan 2003, Li y Chou 2003). Estos valores hacen que los nanotubos se puedan considerar unas 100 veces más resistentes que el acero, por ejemplo. Se han realizado así mismo pruebas de resistencia frente al estiramiento que arrojan resultados del mismo orden de magnitud (Yu et al. 2000a, Yu et al. 2000b). También se han hecho estudios de las propiedades mecánicas cuando en el nanotubo encontramos defectos o deformaciones. En (Sammalkorpi et al. 2004) se estudia cómo afecta al módulo de Young y a la tensión de estiramiento la concentración de defectos tipo hueco en el nanotubo. Los autores obtienen una expresión analítica que relaciona el módulo de Young con la densidad de defectos y muestran cómo la tensión de estiramiento disminuye en un factor 2 por cada hueco en el CNT. 


\subsection{Puntos cuánticos}

Los puntos cuánticos son nanoestructuras en las que los electrones están completamente confinados en las tres dimensiones del espacio (Banyai y Koch 1993, Turton 1995). Existen diversos trabajos que muestran diferente tipo de nanoestructuras formadas por nanotubos de carbono que presentan el comportamiento de puntos cuánticos (Chico et al. 1998).

El hecho de que el tamaño de un punto cuántico sea de orden nanométrico hace que estas estructuras se consideren 0-dimensionales, siendo denominadas también átomos artificiales, ya que los electrones quedan confinados en dichas estructuras del mismo modo en que lo hacen en los orbitales alrededor del núcleo de un átomo. Sin embargo, la importancia de la existencia de nanoestructuras que presentan este comportamiento radica en la posibilidad de manipularlos y trabajar con ellos conectándolos a electrodos u otro tipo de contactos, lo cual se hace imposible para átomos individuales.

Debido a su reducido tamaño, en los puntos cuánticos la energía de los electrones está cuantizada, lo que convierte a estas estructuras en herramientas tremendamente útiles para estudiar una gran cantidad de fenómenos cuánticos: el efecto Hall cuántico en campos magnéticos intensos, el comportamiento atomístico en general o el caos cuántico son algunos ejemplos.

Una propiedad característica de los puntos cuánticos es que al ser iluminados por rayos ultravioleta, emiten luz visible de longitud de onda muy específica, que depende del tamaño de dicho punto.

En cuanto a las posibles aplicaciones industriales, existen numerosas posibilidades en campos tan diversos como la biomedicina, las telecomunicaciones, la optoelectrónica o la computación cuántica. A continuación relacionamos algunas de estas aplicaciones: 
$\square$ Fabricación de diodos láser emisores de luz, que resultan más eficientes que los usados hoy en lectores de CD o DVD.

$\square$ En criptografía cuántica y en seguridad: en billetes o documentos oficiales pueden embeberse puntos cuánticos invisibles a simple vista, pero que se hacen visibles si son iluminados por radiación ultravioleta.

$\square$ En medicina pueden tener aplicaciones diversas en obtención de imágenes con gran contraste, en métodos de tinción o como sondas moleculares. A los puntos cuánticos que tienen más o menos el tamaño de las proteínas, se les puede pegar anticuerpos capaces de reconocer compuestos, células o virus. Muchos investigadores planean usarlos como marcadores de células cancerosas, a las que se podría seguir a medida que se multiplican o migran.

$\square$ En computación cuántica existen numerosos trabajos que proponen utilizar los puntos cuánticos como bits cuánticos. Recientemente un grupo de investigadores del Instituto Kavli de Nanociencia de Holanda, han mostrado una forma de controlar el espín de un solo electrón utilizando un doble punto cuántico. En los ordenadores cuánticos, la unidad de información es el qubit, cuya realización física debe ser un sistema microscópico con dos estados cuánticos accesibles, por ejemplo, un electrón que puede tener su espín arriba o abajo. Estos investigadores han utilizado un método basado en confinar al electrón en un doble punto cuántico, lo que les permite utilizar condiciones menos extremas para controlar el estado cuántico de espín del electrón. En un doble punto cuántico el cambio de espín se puede hacer pasando un electrón de un punto al otro, lo que es más sencillo que relajar al electrón a un estado reserva cuando se usa un solo punto cuántico. Además este método permite una detección del estado de espín mucho menos sensible a las condiciones de medida. Esto abre nuevas vías para permitir todo tipo de operaciones cuánticas en condiciones menos extremas. 


\section{Capítulo 2}

\section{Método de cálculo}

Como sabemos, nuestro estudio se centra en el cálculo de las propiedades de transporte electrónico en nanotubos de carbono. En el capítulo anterior repasamos los conceptos generales sobre dichas estructuras. Dedicaremos este segundo capítulo al desarrollo de las herramientas de cálculo que utilizaremos en la obtención de las propiedades electrónicas de nuestro sistema.

Nuestros cálculos se basan en la aplicación del modelo tight-binding, en el que se considera que sólo existe interacción entre los vecinos próximos del sistema. Así pues, comenzaremos este capítulo explicando en detalle las ecuaciones que se derivan de la aplicación de este modelo.

En segundo lugar, describiremos el proceso de cálculo de la función de Green del sistema (FG). La obtención de dicha función representa una manera muy efectiva de abordar el cálculo de las propiedades de transmisión en cualquier sistema. A partir de ella se pueden obtener diversas magnitudes de interés físico como las amplitudes de reflexión y transmisión, la densidad de estados o el espectro energético. En el apartado 2.4 describiremos en detalle el método numérico de cálculo de la FG. 


\subsection{Modelo tight-binding para sólidos cristalinos}

En una red cristalina, si nos movemos en la dirección de los vectores de la red $\left(\vec{a}_{i}\right.$, donde $i=1,2,3$ para una red tridimensional) nos trasladamos entre puntos equivalentes entre sí dentro de dicha red. Así, cualquier función de onda $\Psi$ satisface el teorema de Bloch (Ashcroft y Mermin 1976, Kittel 1986):

$$
T_{\vec{a}_{i}} \Psi=e^{i \vec{k} \cdot \vec{a}_{i}} \Psi
$$

donde $T_{\vec{a}_{i}}$ es el operador traslacional según los vectores de la red, y $\vec{k}$ el vector de onda, relacionado con la energía y para el cual obtendremos las relaciones de dispersión de la energía en función de dicho vector.

Existen diversas combinaciones de ondas que satisfacen el teorema de Bloch. Sin embargo, de acuerdo con el modelo tight-binding, la función de onda de un sistema se expresa como un conjunto de orbitales atómicos localizados, uno por cada sitio. Por tanto tomaremos como función de onda de Bloch $\Phi_{j}(\vec{k}, \vec{r})$ :

$$
\Phi_{j}(\vec{k}, \vec{r})=\frac{1}{\sqrt{N}} \sum_{\vec{R}}^{N} e^{i \vec{k} \cdot \vec{R}} \varphi_{j}(\vec{r}-\vec{R}), \quad(j=1, \ldots, n),
$$

donde $\vec{R}$ indica la posición de cada átomo, y $\varphi_{j}$ la función de onda del sitio $j$. El número de funciones de onda de una celda unidad se denota como $n$, por lo que se tienen $n$ funciones de Bloch en el cristal por cada valor de $\vec{k}$. El número de celdas unidad dentro del sólido se representa como $N$. Así pues, para formar la función $\Phi_{j}(\vec{k}, \vec{r})$ se realiza la suma ponderada de todas las $\varphi_{j}$ con el peso dado por el factor de fase $e^{i \vec{k} \cdot \vec{R}}$. Dicha suma se realiza sobre todos los vectores de la red del cristal.

Al trasladarnos de una celda unidad a la siguiente, las funciones de Bloch han de ser iguales. Si expresamos esta condición para cada dirección de los vectores de la red podemos escribir:

$$
\Phi_{j}\left(\vec{k}, \vec{r}+M \vec{a}_{i}\right)=\Phi_{j}(\vec{k}, \vec{r}), \quad(i=1,2,3),
$$


donde $M=N^{1 / 3}$ para una red tridimensional. De la condición anterior se deriva que el factor de fase satisface $e^{i k M a_{i}}=1$, y por tanto el número de onda $k$ se puede escribir en función de un número entero $p$ que toma los valores:

$$
k=\frac{2 p \pi}{M a_{i}}, \quad(p=0,1, \ldots, M-1) \quad(i=1,2,3) .
$$

Las autofunciones del sólido $\Psi_{j}(\vec{k}, \vec{r}),(j=1, \ldots, n$, donde $n$ es el número de funciones de Bloch), se expresan como combinación lineal de dichas funciones $\Phi_{j^{\prime}}(\vec{k}, \vec{r})$ :

$$
\Psi_{j}(\vec{k}, \vec{r})=\sum_{j^{\prime}=1}^{n} C_{j j^{\prime}}(\vec{k}) \Phi_{j^{\prime}}(\vec{k}, \vec{r})
$$

donde $C_{j j^{\prime}}(\vec{k})$ son determinados coeficientes que se han de calcular y que dependen del sólido cristalino considerado. Las funciones de onda del cristal también satisfacen el teorema de Bloch, por lo que la suma se extiende únicamente sobre las funciones $\Phi_{j^{\prime}}(\vec{k}, \vec{r})$ con el mismo valor de $\vec{k}$.

La autoenergía $E_{j}(\vec{k}),(j=1, \ldots, n)$ asociada a la función de onda $\vec{k}$ viene dada por la exprexión:

$$
E_{j}(\vec{k})=\frac{\left\langle\Psi_{j}|\hat{H}| \Psi_{j}\right\rangle}{\left\langle\Psi_{j} \mid \Psi_{j}\right\rangle}=\frac{\int \Psi_{j}^{*} \hat{H} \Psi_{j} d \vec{r}}{\int \Psi_{j}^{*} \Psi_{j} d \vec{r}},
$$

donde $\hat{H}$ es el hamiltoniano del cristal. Si se sustituye la ecuación (2.5) en (2.6) se obtiene:

$$
E_{j}(\vec{k})=\frac{\sum_{j, j^{\prime}=1}^{n} C_{i j}^{*} C_{i j^{\prime}}\left\langle\Phi_{j}|\hat{H}| \Phi_{j^{\prime}}\right\rangle}{\sum_{j, j^{\prime}=1}^{n} C_{i j}^{*} C_{i j^{\prime}}\left\langle\Phi_{j} \mid \Phi_{j^{\prime}}\right\rangle} .
$$

Podemos definir las expresiones integrales sobre los orbitales de Bloch en (2.7) como $H_{j j^{\prime}}(\vec{k})$ y $S_{j j^{\prime}}(\vec{k})$, denominadas matrices de transferencia (o Hamiltoniano) 
y de solapamiento, respectivamente, que por tanto quedan definidas como:

$$
\begin{gathered}
H_{j j^{\prime}}(\vec{k})=\left\langle\Phi_{j}|\hat{H}| \Phi_{j^{\prime}}\right\rangle \quad\left(j, j^{\prime}=1, \ldots n\right), \\
S_{j j^{\prime}}(\vec{k})=\left\langle\Phi_{j} \mid \Phi_{j^{\prime}}\right\rangle \quad\left(j, j^{\prime}=1, \ldots n\right) .
\end{gathered}
$$

Como hemos mencionado anteriormente, en el modelo tight-binding sólo se considera que existe interacción entre vecinos próximos, despreciando las posibles interacciones entre segundos vecinos. Por lo tanto, en las expresiones anteriores sólo serán diferentes de cero los elementos diagonales de las matrices, así como aquellos elementos para los que los índices $j, j^{\prime}$ se correspondan con vecinos próximos.

Con las definiciones de $H_{j j^{\prime}}$ y $S_{j j^{\prime}}$ dadas por (2.8) y (2.9) la expresión (2.7) queda como:

$$
E_{j}(\vec{k})=\frac{\sum_{j, j^{\prime}=1}^{n} H_{j j^{\prime}}(\vec{k}) C_{i j}^{*} C_{i j^{\prime}}}{\sum_{j, j^{\prime}=1}^{n} S_{j j^{\prime}}(\vec{k}) C_{i j}^{*} C_{i j^{\prime}}} .
$$

Los coeficientes $C_{j j^{\prime}}^{*}(\vec{k})$ se ajustan de manera que la autoenergía $E_{i}(\vec{k})$ sea la mínima posible. Por tanto, la derivada parcial de $E_{i}(\vec{k})$ respecto a dichos coeficientes ha de ser cero. Al derivar mantenemos constantes los valores de los demás coeficientes $\left(C_{i j^{\prime}}, C_{i j^{\prime}}^{*}\right.$ y $\left.C_{i j}\right)$. La expresión correspondiente queda como:

$$
\frac{\partial E_{i}(\vec{k})}{\partial C_{i j}^{*}}=\frac{\sum_{j^{\prime}=1}^{N} H_{j j^{\prime}}(\vec{k}) C_{i j^{\prime}}}{\sum_{j, j^{\prime}=1}^{N} S_{j j^{\prime}}(\vec{k}) C_{i j}^{*} C_{i j^{\prime}}}-\frac{\sum_{j, j^{\prime}=1}^{N} H_{j j^{\prime}}(\vec{k}) C_{i j}^{*} C_{i j^{\prime}}}{\left(\sum_{j, j^{\prime}=1}^{N} S_{j j^{\prime}}(\vec{k}) C_{i j}^{*} C_{i j^{\prime}}\right)^{2}} \sum_{j^{\prime}=1}^{N} S_{j j^{\prime}}(\vec{k}) C_{i j^{\prime}}=0 .
$$

Si multiplicamos la expresión anterior por $\sum_{j, j^{\prime}=1}^{N} S_{j j^{\prime}}(\vec{k}) C_{i j}^{*} C_{i j^{\prime}}$ a ambos lados de 
la igualdad, y sustituimos la autoenergía por la expresión (2.10) obtenemos:

$$
\sum_{j^{\prime}=1}^{N} H_{j j^{\prime}}(\vec{k}) C_{i j^{\prime}}=E_{i}(\vec{k}) \sum_{j^{\prime}=1}^{N} S_{j j^{\prime}}(\vec{k}) C_{i j^{\prime}}
$$

expresión que queda reducida a:

$$
\hat{H} \vec{C}_{i}=E_{i}(\vec{k}) \hat{S} \vec{C}_{i} \quad \text { o bien } \quad\left[\hat{H}-E_{i}(\vec{k}) \hat{S}\right] \vec{C}_{i}=0
$$

donde se define el vector columna $\vec{C}_{i}$ :

$$
\vec{C}_{i}=\left(\begin{array}{c}
C_{i 1} \\
\vdots \\
C_{i N}
\end{array}\right)
$$

Si existe la inversa de la matriz $\left[\hat{H}-E_{i}(\vec{k}) \hat{S}\right]$ en la expresión $(2.13)$, se pueden obtener los coeficientes $\vec{C}_{i}$ multiplicando a ambos lados por $\left[\hat{H}-E_{i}(\vec{k}) \hat{S}\right]^{-1}$, con lo que tendríamos que $\vec{C}_{i}=0$. Así pues, las autofunciones existen únicamente si dicha matriz no tiene inversa, lo cual es equivalente a que el determinante de la matriz sea cero:

$$
\operatorname{det}[\hat{\mathrm{H}}-\mathrm{E} \hat{\mathrm{S}}]=0 \text {. }
$$

Esta expresión, conocida como ecuación secular, es la que utilizamos en el apartado 1.5 del capítulo anterior para obtener la estructura electrónica de los CNT, determinando la región de Brillouin y la red recíproca, una vez seleccionados los puntos de simetría en dicha región.

\subsubsection{Aplicación a CNT. Función de participación}

En el apartado 1.5 ya especificamos que para aplicar el modelo tight-binding a nuestro sistema, lo supondríamos formado por átomos con un único orbital $\pi, \mathrm{y}$ con una energía de solapamiento igual a $t=-2,66 \mathrm{eV}$ (Blase et al. 1994). Existen 
múltiples referencias que ponen de manifiesto que los resultados así obtenidos se corresponden muy bien con otros métodos más sofisticados que tienen en cuenta más orbitales por átomo (Orlikowski et al. 2001). Por otro lado, tampoco tendremos en cuenta los efectos de la curvatura del nanotubo, ya que dichos efectos sólo cobran importancia para nanotubos de diámetros muy pequeños, con los que no vamos a trabajar en esta memoria (Blase et al. 1994, Zolyomi y Kürti 2004).

Para obtener las magnitudes asociadas al transporte electrónico en nanotubos partiremos del cálculo de la función de Green del sistema, procedimiento que se describe en el apartado 2.4. En el cálculo de dicha función tendremos en cuenta que el nanotubo ha de estar conectado por ambos extremos a dos contactos, leads. Sin embargo, en una primera aproximación, no es necesario tener en cuenta dichos contactos si el nanotubo considerado es lo suficientemente largo. En este caso podemos obtener las autofunciones correspondientes simplemente por diagonalización del hamiltoniano tight-binding.

A través de las autofunciones podremos calcular el valor de la función de participación $P_{j}$. Esta función nos permite conocer el grado de localización de una función de onda. Pasamos a explicar cómo obtener esta función de participación. El hamiltoniano del nanotubo se representa a través de una matriz $N \times N$, donde $N$ es el número de átomos en el mismo. Vamos a considerar la energía de sitio igual a cero, con lo que los elementos diagonales del hamiltoniano son todos cero. Únicamente tendremos en cuenta la interacción entre vecinos próximos. Así pues, los elementos de la matriz del hamiltoniano distintos de cero son aquellos que conectan átomos enlazados entre sí, cuyo valor viene dado por la energía de solapamiento. Al diagonalizar el hamiltoniano obtenemos las $N$ autoenergías y $N$ autovectores de dicha matriz. Cada uno de estos autovectores tiene, a su vez, $N$ componentes, que se relacionan con la amplitud de la función de onda en el sitio correspondiente. Denotaremos a las autoenergías como $E_{j}$, y a las autofunciones como $\Psi_{j}(m)$, donde $j, m=1, \cdots, N$. La función de participación $P_{j}$ se define a través de su inversa como (Wegner 1980): 


$$
P_{j}^{-1}=\sum_{m}\left|\Psi_{j}(m)\right|^{4} .
$$

La función de participación nos da una medida de la región del sistema en la que la amplitud de la función de onda es diferente de cero, así que nos permite conocer si los estados que representa cada autofunción son extendidos o localizados. Podemos tratar de entender la expresión (2.16) en los límites extremos: onda totalmente localizada y onda totalmente extendida. Dado que las funciones de onda están normalizadas, para una onda completamente localizada en un sólo átomo las componentes de la función de onda ha de ser todas cero excepto aquella donde se encuentre localizada dicha función, cuyo valor será igual a uno. En este caso, según la definición (2.16) el valor de la función de participación es igual a uno. Para una onda totalmente extendida a lo largo de un sistema compuesto por $N$ átomos, todas las componentes de dicha onda han de ser del orden de $1 / \sqrt{N}$, por lo que el valor de la función de participación es en este caso aproximadamente igual al número de átomos del sistema. Es decir:

$$
\begin{array}{ll}
\text { Estado completamente localizado: } & \Psi_{j}(m) \sim \delta_{j m} \Rightarrow P_{j}=1, \\
\text { Estado completamente extendido: } & \Psi_{j}(m) \sim \frac{1}{\sqrt{N}} \Rightarrow P_{j} \approx N .
\end{array}
$$

En los casos intermedios, podemos considerar que el valor de la función de participación es el número de átomos sobre los que se extiende la función de onda correspondiente. Así pues, utilizaremos el cálculo de la función de participación como herramienta para conocer la naturaleza extendida o localizada de las funciones de onda en nuestro sistema. 


\subsection{Función de Green. Definición}

El cálculo de la función de Green (FG) del sistema constituye sin duda el núcleo central imprescindible para la obtención de todas las magnitudes estudiadas en la presente memoria. Así pues exponemos en este apartado su definición, que nos permitirá posteriormente establecer un método de cálculo para dicha función en nuestro sistema, y su relación con las magnitudes estudiadas en esta tesis.

La FG es un concepto muy versátil. Puede interpretarse como un propagador de un punto a otro o como una función de respuesta. Es igual de clara y significativa en física clásica como en mecánica cuántica, incluso en la más complicada teoría cuántica de campos. Además es un objeto muy útil para la realización de cálculos prácticos (Economou 1983, Datta 1995).

Dada una función de onda $\Psi(\vec{r})$ que cumple la ecuación de Schrödinger independiente del tiempo:

$$
[E-H(\vec{r})] \Psi(\vec{r})=0
$$

donde $H$ es el operador hamiltoniano del sistema y $E$ la energía, se define la Función de Green $G\left(\vec{r}, \overrightarrow{r^{\prime}} ; E\right)$ mediante la siguiente expresión:

$$
[E-H(\vec{r})] G\left(\vec{r}, \vec{r}^{\prime} ; E\right)=\delta\left(\vec{r}-\vec{r}^{\prime}\right)
$$

Por tanto, se puede interpretar la función de Green como la función de onda en $\vec{r}$ debida a una excitación producida en $\vec{r}^{\prime}$. Podemos suprimir en nuestra notación la dependencia espacial, escribiendo así la FG como $G(E)$. En esta notación, la expresión anterior es equivalente a la siguiente ecuación diferencial independiente del tiempo:

$$
G(E)=[E-H]^{-1}
$$


Según la expresión (2.20), la FG se define como la inversa de un operador diferencial. En este caso la solución no queda determinada completamente hasta que no se imponen las condiciones de frontera del sistema. Aparecen como solución dos operadores de Green diferentes, el retardado y el avanzado. El proceso de cálculo podemos verlo mediante el ejemplo que desarrollamos a continuación, en el que consideramos un conductor infinito unidimensional a potencial es constante ( $U=U_{0}=$ constante). Si el conductor se encuentra sobre el eje $x$, esribimos la ecuación (2.20):

$$
G(E)=\left[E-U_{0}+\frac{\hbar^{2}}{2 m} \frac{\partial^{2}}{\partial x^{2}}\right]^{-1}
$$

o lo que es lo mismo:

$$
\left[E-U_{0}+\frac{\hbar^{2}}{2 m} \frac{\partial^{2}}{\partial x^{2}}\right] G\left(x, x^{\prime} ; E\right)=\delta\left(x-x^{\prime}\right) .
$$

Debido a la propia definición de función de Green, la ecuación (2.22) es similar a la ecuación de Schrödinger para la función de onda:

$$
\left[E-U_{0}+\frac{\hbar^{2}}{2 m} \frac{\partial^{2}}{\partial x^{2}}\right] \Psi(x)=0
$$

Como ya hemos mencionado, la función de Green representa la función de onda en $x$ debida a una excitación producida en $x^{\prime}$. Físicamente, dicha excitación da lugar a dos ondas que viajan alejándose de la fuente en $x^{\prime}$, hacia la derecha y hacia la izquierda, ondas con amplitudes que denotaremos mediante $A^{+} \mathrm{y} A^{-}$, con lo que escribimos:

$$
\begin{aligned}
& G\left(x, x^{\prime} ; E\right)=A^{+} e^{\left[i k\left(x-x^{\prime}\right)\right]} \quad \text { si } \quad x>x^{\prime}, \\
& G\left(x, x^{\prime} ; E\right)=A^{-} e^{\left[-i k\left(x-x^{\prime}\right)\right]} \text { si } \quad x<x^{\prime} .
\end{aligned}
$$

donde $k=\sqrt{2 m\left(E-U_{0}\right)} / \hbar$. 
Los valores para $A^{+}$y $A^{-}$se obtienen sustituyendo por $x=x^{\prime}$ en la ecuación (2.22). La función de Green es continua y su primera derivada es discontinua y con un salto dado por $2 m / \hbar^{2}$, es decir:

$$
\begin{gathered}
{\left[G\left(x, x^{\prime} ; E\right)\right]_{x=x^{\prime}}=\left[G\left(x, x^{\prime} ; E\right)\right]_{x=x^{\prime-}}} \\
{\left[\frac{\partial G\left(x, x^{\prime} ; E\right)}{\partial x}\right]_{x=x^{+}}-\left[\frac{\partial G\left(x, x^{\prime} ; E\right)}{\partial x}\right]_{x=x^{\prime}}=\frac{2 m}{\hbar^{2}}}
\end{gathered}
$$

Así, se obtienen los valores de $A^{+}$y $A^{-}$sustituyendo la expresión (2.24) en (2.25) y $(2.26)$ :

$$
A^{+}=A^{-}=-\frac{i}{\hbar v} \quad \text { donde } \quad v=\frac{\hbar k}{m} .
$$

Se tienen así dos soluciones para la función de Green:

$$
\begin{aligned}
G^{R}\left(x, x^{\prime} ; E\right) & =-\frac{i}{\hbar v} e^{i k\left|x-x^{\prime}\right|} \\
G^{A}\left(x, x^{\prime} ; E\right) & =+\frac{i}{\hbar v} e^{-i k\left|x-x^{\prime}\right|} .
\end{aligned}
$$

Las dos funciones anteriores son soluciones de la ecuación (2.22) pero con diferentes condiciones de frontera. El operador retardado $\left(G^{R}\right)$ se corresponde con las ondas salientes de $x^{\prime}$, mientras que el avanzado $\left(G^{A}\right)$ son las ondas entrantes. Puede verse con este ejemplo que la relación entre ambos es la siguiente:

$$
G^{A}\left(x, x^{\prime} ; E\right)=G^{R}\left(x^{\prime}, x ; E\right)^{*}
$$

relación que es aplicable a cualquier sistema.

Otro procedimiento consiste en incorporar las condiciones de frontera en la propia ecuación de definición de la función de Green, sumando una parte imaginaria 
infinitesimal a la energía. Para ello se introduce un parámetro $\eta \rightarrow 0^{+}$. Así, en lugar de la ecuación (2.22) tenemos dos ecuaciones, una para el operador retardado y otra para el operador avanzado:

$$
\begin{gathered}
{\left[E-U_{0}+\frac{\hbar^{2}}{2 m} \frac{\partial^{2}}{\partial x^{2}}+i \eta\right] G^{R}\left(x, x^{\prime} ; E\right)=\delta\left(x-x^{\prime}\right),} \\
{\left[E-U_{0}+\frac{\hbar^{2}}{2 m} \frac{\partial^{2}}{\partial x^{2}}-i \eta\right] G^{A}\left(x, x^{\prime} ; E\right)=\delta\left(x-x^{\prime}\right) .}
\end{gathered}
$$

En lo que sigue cuando hablemos de la función de Green nos referiremos al operador retardado, por lo que no será necesario utilizar el superíndice $R$ en la notación. La definición más general para el operador de Green retardado es la siguiente:

$$
G(E)=[E-H+i \eta]^{-1} \quad\left(\eta \rightarrow 0^{+}\right) .
$$

Si definimos en la expresión (2.33) un nuevo parámetro $\varepsilon=E+i \eta$ complejo, podemos reescribir dicha expresión como:

$$
G(\varepsilon)=[\varepsilon-H]^{-1},
$$

o también como:

$$
[\varepsilon-H(x)] G\left(x, x^{\prime} ; \varepsilon\right)=\delta\left(x-x^{\prime}\right) .
$$

Todo el formalismo anterior es aplicable a sistemas tridimensionales. La extensión a dichos sistemas se realiza de forma trivial, reemplazando las coordenadas escalares $x, x^{\prime}$ por coordenadas vectoriales $\vec{r}, \vec{r}^{\prime}$. Así, la ecuación (2.35) se escribe finalmente:

$$
[\varepsilon-H(\vec{r})] G\left(\vec{r}, \vec{r}^{\prime} ; \varepsilon\right)=\delta\left(\vec{r}-\vec{r}^{\prime}\right) .
$$


En el caso en que tengamos un sistema discreto todas las expresiones anteriores se discretizan, convirtiéndose entonces tanto la función de Green como el hamiltoniano del sistema en matrices, que denotaremos mediante el símbolo ^ encima de la letra que representa a la matriz. La expresión (2.36) se escribe en este caso:

$$
[\varepsilon \hat{I}-\hat{H}] \hat{G}(\epsilon)=\hat{I}
$$

o bien:

$$
\hat{G}(\epsilon)=[\varepsilon \hat{I}-\hat{H}]^{-1}
$$

\subsubsection{Relación con las funciones de onda}

Se puede obtener también una expresión de la FG a partir de las funciones de onda, ya que la definición de función de Green se relaciona con la ecuación de Schrödinger, como mencionamos al principio de este apartado. Puesto que las autofunciones del operador hamiltoniano forman un conjunto ortonormal y completo, la FG se puede expresar como combinación lineal de estas funciones de onda, es decir:

$$
G\left(\vec{r}, \vec{r}^{\prime} ; E\right)=\sum_{\alpha} C_{\alpha}\left(\vec{r}^{\prime}\right) \Psi_{\alpha}(\vec{r})
$$

Para determinar los coeficientes $C_{\alpha}\left(\vec{r}^{\prime}\right)$ sustituimos la expresión anterior en la ecuación de la FG (2.36), haciendo uso así mismo de la relación (2.18). Si representamos el conjunto de autoenergías asociadas a las funciones de onda como $\epsilon_{\alpha}$ podemos reescribir la expresión (2.36):

$$
\sum_{\alpha}\left(E-\epsilon_{\alpha}+i \eta\right) C_{\alpha}\left(\vec{r}^{\prime}\right) \Psi_{\alpha}(\vec{r})=\delta\left(\vec{r}-\vec{r}^{\prime}\right)
$$

Si multiplicamos la expresión $(2.40)$ por $\Psi_{\alpha}^{*}(\vec{r})$ e integramos con respecto a $\vec{r}$, haciendo uso de la relación de ortogonalidad de las autofunciones, obtenemos los 
coeficientes $C_{\alpha}\left(\vec{r}^{\prime}\right)$ :

$$
C_{\alpha}\left(\vec{r}^{\prime}\right)=\frac{\Psi_{\alpha}^{*}\left(\vec{r}^{\prime}\right)}{E-\epsilon_{\alpha}+i \eta} .
$$

Finalmente, sustituyendo la expresión de los coeficientes $C_{\alpha}\left(\vec{r}^{\prime}\right)$ en la ecuación (2.39), obtenemos la relación que buscamos entre la función de Green y las funciones de onda:

$$
G\left(\vec{r}, \vec{r}^{\prime} ; E\right)=\sum_{\alpha} \frac{\Psi_{\alpha}(\vec{r}) \Psi_{\alpha}^{*}\left(\vec{r}^{\prime}\right)}{E-\epsilon_{\alpha}+i \eta}
$$

\subsubsection{Aplicación a sistemas finitos. Autoenergías}

Las expresiones (2.37) y (2.38) son aplicables a sistemas discretos infinitos. Esto es, las matrices que aparecen en dichas expresiones son de dimensión infinita. Para poder aplicarlas a sistemas reales es necesario tener en cuenta las fronteras del sistema, que pueden producir reflexiones en las mismas que no se consideran en el caso de sistemas infinitos. Para ello, se ha de conectar el sistema a dos contactos semi-infinitos en ambos extremos. Este proceso se lleva a cabo separando la función de Green del sistema en las diferentes partes correspondientes a los contactos $\left(\hat{G}_{l}\right)$, al sistema $\left(\hat{G}_{s}\right)$ y a la interacción entre ambos $\left(\hat{G}_{l s}\right.$ y $\left.\hat{G}_{s l}\right)$. Así, reescribimos la expresión (2.38):

$$
\left(\begin{array}{cc}
\hat{G}_{l} & \hat{G}_{l s} \\
\hat{G}_{s l} & \hat{G}_{s}
\end{array}\right)=\left(\begin{array}{cc}
(E+i \eta) \hat{I}-\hat{H}_{l} & \hat{\tau}_{l} \\
\hat{\tau}_{l}^{\dagger} & E \hat{I}-\hat{H}_{s}
\end{array}\right)^{-1}
$$

En el elemento correspondiente al sistema no es necesario añadir el parámetro complejo infinitesimal, ya que dicho parámetro se puede englobar en el término correspondiente al contacto, como veremos en el desarrollo a continuación. La matriz $\hat{\tau}_{l}$ es la matriz de acoplamiento entre el contacto y el sistema. Hemos de tener en cuenta que los contactos pueden tener más de un modo de transmisión con el sistema. Así pues, la matriz $\hat{\tau}_{l}$ tiene diferentes de cero todos elementos correspondientes a 
la interacción entre puntos adyacentes entre el contacto y el sistema. El valor de dichos elementos es el correspondiente a la energía de interacción entre ambos, que denotaremos como $t$. Así pues:

$$
\hat{\tau}_{l}\left(i^{\prime}, j\right)=\left\{\begin{array}{lll}
t & \text { si } & i^{\prime}=j, \\
0 & \text { si } & i^{\prime} \neq j,
\end{array}\right.
$$

donde denotamos con ' los índices correspondientes a los elementos del contacto, que se etiquetan de manera que el valor de los índices entre puntos adyacentes del sistema y el contacto sea el mismo. Ampliaremos el concepto de transmisión multimodos en el apartado 2.2.3.

Para obtener la función de Green del sistema desarrollamos la expresión (2.43):

$$
\begin{gathered}
{\left[(E+i \eta) \hat{I}-\hat{H}_{l}\right] \hat{G}_{l s}+\left[\hat{\tau}_{l}\right] \hat{G}_{s}=0,} \\
{\left[E \hat{I}-\hat{H}_{s}\right] \hat{G}_{s}+\left[\hat{\tau}_{l}^{\dagger}\right] \hat{G}_{l s}=\hat{I} .}
\end{gathered}
$$

De la expresión (2.45) se obtiene:

$$
\hat{G}_{l s}=-\hat{g}_{l} \hat{\tau}_{l} \hat{G}_{s}
$$

donde $\hat{g}_{l}$ se define como la función de Green del contacto semi-infinito:

$$
\hat{g}_{l}=\left[(E+i \eta) \hat{I}-\hat{H}_{l}\right]^{-1}
$$

Sustituyendo la expresión (2.47) en (2.46) se obtiene para la función de Green del sistema:

$$
\hat{G}_{s}=\left[E \hat{I}-\hat{H}_{s}-\hat{\tau}_{l}^{\dagger} \hat{g}_{l} \hat{\tau}_{l}\right]^{-1}
$$

La matriz $\hat{G}_{s}$ es de dimensión $N \times N$, donde $N$ es el número de puntos del sistema. Aunque el término $\hat{\tau}_{l}^{\dagger} \hat{g}_{l} \hat{\tau}_{l}$ representa al contacto semi-infinito, no es necesario invertir una matriz infinita en su cálculo. Ya que dicho término representa al contacto aislado, 
la función de Green correspondiente se puede obtener de forma analítica. Teniendo en cuenta el valor de $\hat{\tau}_{l}$ dado por la expresión (2.44) podemos escribir el término $\hat{\tau}_{l}^{\dagger} \hat{g}_{l} \hat{\tau}_{l}$ como:

$$
\left[\hat{\tau}_{l}^{\dagger} \hat{g}_{l} \hat{\tau}_{l}\right]_{i j}=t^{2} \hat{g}_{l}\left(i^{\prime}, j^{\prime}\right)
$$

Finalmente, podemos suponer que los efectos de la conexión de varios contactos al sistema es aditivo, con lo que obtenemos:

$$
\hat{G}(E)=\left[E \hat{I}-\hat{H}_{s}-\hat{\Sigma}\right]^{-1},
$$

donde se ha definido la matriz de autoenergías del sistema $\hat{\Sigma}$ como:

$$
\hat{\Sigma}=\sum_{k} \hat{\Sigma}_{k} \quad, \quad \hat{\Sigma}_{k}(i, j)=t^{2} \hat{g}_{l}\left(i^{\prime}, j^{\prime}\right) .
$$

La suma se extiende sobre todos los contactos en conexión con el sistema. En nuestro trabajo conectaremos el nanotubo únicamente a dos contactos semi-infinitos, a derecha e izquierda del mismo. Así pues, escribiremos la expresión anterior como:

$$
\hat{G}(E)=\left[E \hat{I}-\hat{H}_{s}-\hat{\Sigma}_{L}-\hat{\Sigma}_{R}\right]^{-1}
$$

donde $\hat{\Sigma}_{L}$ y $\hat{\Sigma}_{R}$ son los términos de autoenergías correspondientes a los contactos a izquierda y derecha, respectivamente. En esta expresión denotamos la función de Green final del sistema como $\hat{G}$, que representa la propagación de los electrones entre dos puntos teniendo en cuenta los efectos de la conexión de los contactos a través del término de autoenergías.

\subsubsection{Sistemas multi-modos}

En este apartado vamos a obtener una expresión para la función de Green de un sistema con varios modos de transmisión. Supongamos un sistema bidimensional 
infinito a lo largo del eje $x$ pero de longitud finita a lo largo del eje $y$. Estudiamos la función de onda en $(x, y)$ como consecuencia de una excitación producida en $\left(x^{\prime}, y^{\prime}\right)$ a través de la FG $G\left(x, y ; x^{\prime}, y^{\prime} ; E\right)$. La onda correspondiente ha de ser una superposición de los diferentes modos de transmisión sobre el eje $Y$. Escribiremos la función de Green correspondiente como:

$$
G\left(x, x^{\prime} ; E\right)=\sum_{m} A_{m}^{ \pm} \chi_{m}(y) e^{i k\left|x-x^{\prime}\right|}
$$

donde $A_{m}^{ \pm}$son las amplitudes de los diferentes modos con que se propaga la onda desde el origen de la excitación. Denotamos las funciones de los modos transversales como $\chi_{m}(y)$, que satisfacen la ecuación:

$$
\left[-\frac{\hbar^{2}}{2 m} \frac{\partial^{2}}{\partial y^{2}}+U(y)\right] \chi_{m}(y)=\epsilon_{m, 0} \chi_{m}(y),
$$

donde $U(y)$ es el potencial transversal a lo largo del eje $y$. Las funciones de los modos transversales han de cumplir la relación de ortogonalidad entre ellas. Trabajaremos con la aproximación de que dichas funciones son reales. Para calcular la amplitud de los modos transversales, $A_{m}^{ \pm}$, procedemos de la misma forma que en el apartado 2.2, donde obtuvimos las amplitudes de una onda unidimensional. Es decir, imponemos las condiciones de continuidad en la FG y discontinuidad de salto en su primera derivada:

$$
\begin{gathered}
{\left[G\left(x, x^{\prime} ; E\right)\right]_{x=x^{\prime}}=\left[G\left(x, x^{\prime} ; E\right)\right]_{x=x^{\prime-}}} \\
{\left[\frac{\partial G\left(x, x^{\prime} ; E\right)}{\partial x}\right]_{x=x^{\prime+}}-\left[\frac{\partial G\left(x, x^{\prime} ; E\right)}{\partial x}\right]_{x=x^{\prime-}}=\frac{2 m}{\hbar^{2}} \delta\left(y-y^{\prime}\right) .}
\end{gathered}
$$

Sustituyendo la expresión (2.54) en (2.56) y (2.57) obtenemos:

$$
\sum_{m} A_{m}^{+} \chi_{m}(y)=\sum_{m} A_{m}^{-} \chi_{m}(y)
$$




$$
\sum_{m} i k_{m}\left[A_{m}^{+}+A_{m}^{-}\right] \chi_{m}(y)=\frac{2 m}{\hbar^{2}} \delta\left(y-y^{\prime}\right) .
$$

Si multiplicamos las expresiones (2.58) y (2.59) por $\chi_{n}(y)$, integramos y tenemos en cuenta la relación de ortogonalidad para las funciones de onda transversales, obtenemos:

$$
A_{m}^{+}=A_{m}^{-} \quad \text { y } \quad i k_{m}\left[A_{m}^{+}+A_{m}^{-}\right]=\frac{2 m}{\hbar^{2}} \chi\left(y^{\prime}\right) .
$$

Así pues, el valor de las amplitudes de los modos transversales viene dado por:

$$
A_{m}^{+}=A_{m}^{-}=-\frac{i}{\hbar v_{m}} \chi_{m}\left(y^{\prime}\right) .
$$

Finalmente, sustituyendo la ecuación (2.61) en (2.54) obtenemos la función de Green:

$$
G\left(x, y ; x^{\prime}, y^{\prime} ; E\right)=\sum_{m}-\frac{i}{\hbar v_{m}} \chi_{m}\left(y^{\prime}\right) \chi_{m}\left(y^{\prime}\right) e^{i k\left|x-x^{\prime}\right|},
$$

donde se han definido $k_{m}=\frac{\sqrt{2 m\left(E-\epsilon_{m, 0}\right)}}{\hbar}$ y $v_{m}=\frac{\hbar k_{m}}{m}$.

En cuanto a la función de autoenergías en un sistema multi-modos, se puede demostrar que para un contacto cualquiera discreto y semi-infinito, dicha función se puede expresar como:

$$
\Sigma_{p}(i, j)=-t \sum_{m \in p} \chi_{m}\left(p_{i}\right) e^{i k_{m} a} \chi_{m}\left(p_{j}\right) .
$$

\subsection{Función de Transmisión y Densidad de Estados}

Una vez calculada la función de Green de un sistema, se pueden obtener a través de la misma numerosas propiedades realcionadas con dicho sistema. Nos ocuparemos en este apartado del método de obtención de la función de transmisión y la densidad de estados. 
La función de transmisión del sistema se obtiene a partir de la matriz de dispersión Scattering Matrix, que denotaremos como $\hat{S_{C}}$. La matriz de dispersión relaciona las amplitudes de las ondas salientes con las amplitudes de las ondas entrantes en el sistema. La probabilidad de transmisión entre dos sitios $i$ y $j$ se obtiene directamente a partir de la matriz $\hat{S_{C}}$ como el cuadrado del elemento correspondiente de dicha matriz, es decir:

$$
T_{i j}=\left|\left(\hat{S}_{C}\right)_{i j}\right|^{2}
$$

Para obtener la función de transmisión en un sistema multi-modos con varios contactos, hemos de sumar todas las amplitudes de transmisión de los modos que conectan las dos regiones consideradas del sistema:

$$
T=\sum_{i^{\prime}} \sum_{j^{\prime}} T_{i^{\prime} j^{\prime}}
$$

De esta manera, para obtener la función de transmisión hemos de calcular primero la matriz de dispersión del sistema. Vamos en primer lugar a obtener una relación entre la matriz $\hat{S_{C}}$ y la función de Green en un sistema es unidimensional, basándonos en las expresiones que obtuvimos en el apartado 2.2. Consideraremos nuestro sistema conectado a dos contactos cualesquiera $p$ y $q$. Mediante la función de Green podemos estudiar la propagación de las ondas en cualquiera de los dos sentidos posibles entre los contactos, así como los efectos de la reflexión en uno sólo de ellos. Supongamos por ejemplo que estudiamos la propagación de la onda de izquierda a derecha del sistema. Para obtener la función de Green correspondiente tendremos que sumar las contribuciones de dos ondas: la que se aleja del conductor dirigiéndose hacia la izquierda, y la que se acerca al mismo dirigiéndose hacia la derecha. Pero esta última sufre reflexión en el contacto de la derecha, lo que hace que su amplitud venga modulada por la matriz $\hat{S_{C}}$. En general, cualquiera que sea el sentido de propagación de la onda, podemos escribir: 


$$
G_{q p}=\delta_{q p} A_{p}^{-}+\left(\hat{S}_{C}^{\prime}\right)_{q p} A_{p}^{+} .
$$

$G_{q p}$ es la función de onda en $q$ debida a una excitación en $p$. La matriz $\hat{S}_{C}^{\prime}$, que aparece en la expresión (2.66) tiene en cuenta únicamente la amplitud de la función de onda. Esta nueva definición viene motivada por el hecho de que la corriente asociada a una onda reflejada es porporcional al cuadrado de la función de onda multiplicada por su velocidad. Así, definimos $\hat{S}_{C}^{\prime}$ a través de la expresión:

$$
\left(\hat{S}_{C}^{\prime}\right)_{q p}=\sqrt{\frac{v_{p}}{v_{q}}}\left(\hat{S}_{C}\right)_{q p}
$$

Las expresiones de $A_{p}{ }^{-}$y $A_{p}{ }^{+}$vienen dadas por la ecuación (2.27). Sustituyendo el valor de $A_{p}{ }^{-}$y $A_{p}{ }^{+}$en la expresión (2.66) y teniendo en cuenta la relación entre $\hat{S}_{C}$ y $\hat{S}_{C}^{\prime}(2.67)$, obtenemos el valor de los elementos de la matriz $\hat{S}_{C}$ :

$$
\left(\hat{S}_{C}\right)_{q p}=-\delta_{q p}+i \hbar \sqrt{v_{q} v_{p}} G_{q p},
$$

expresión que relaciona la matriz $\hat{S}_{C}$ con la función de Green para un sistema unidimensional con dos contactos. Si queremos generalizar esta expresión para el caso de un sistema con varios modos de transmisión, hemos de reemplazar la ecuación (2.66) por otra que incluya la suma sobre todos los posibles modos de transmisión, teniendo en cuenta los correspondientes modos transversales:

$$
G_{q p}\left(y_{q} ; y_{p}\right)=\sum_{m \epsilon p} \sum_{n \in q}\left[\delta_{n m} A_{m}^{-}+\left(\hat{S}_{C}^{\prime}\right)_{n m} A_{m}{ }^{+}\right] \chi_{n}\left(y_{q}\right) .
$$

Sustituyendo en la expresión (2.69) las amplitudes $A_{m}{ }^{+}$y $A_{m}{ }^{-}$por su valor en función de los modos transversales (ecuación 2.61), y teniendo en cuenta (2.67) podemos reescribir la función de Green:

$$
G_{q p}\left(y_{q} ; y_{p}\right)=\sum_{m \in p} \sum_{n \in q}-\frac{i}{\hbar \sqrt{v_{n} v_{m}}}\left[\delta_{n m} A_{m}{ }^{-}+\left(\hat{S}_{C}^{\prime}\right)_{n m} A_{m}{ }^{+}\right] \chi_{n}\left(y_{q}\right)\left[\delta_{n m}+\left(\hat{S}_{C}^{\prime}\right)_{n m}\right] \chi_{m}\left(y_{p}\right) \text {. }
$$


Finalmente, para obtener los elementos de la matriz de dispersión $\hat{S}_{C}$ multiplicamos la expresión (2.70) por $\chi_{m}\left(y_{p}\right) \chi_{n}\left(y_{q}\right)$, e integramos con respecto a $y_{p}$ e $y_{q}$, haciendo uso de la relación de ortogonalidad de las funciones transversales. Así:

$$
\left(\hat{S}_{C}\right)_{n m}=-\delta_{n m}+i \hbar \sqrt{v_{n} v_{m}} \iint \chi_{n}\left(y_{q}\right)\left[G_{q p}\left(y_{q} ; y_{p}\right)\right] \chi_{m}\left(y_{p}\right) d y_{q} d y_{p}
$$

La expresión anterior se conoce como relación de Fisher-Lee. A partir de ella vamos a obtener la función de transmisión del sistema. Para ello, como mencionamos al comienzo del presente apartado, únicamente hemos de elevar al cuadrado los elementos de la matriz $\hat{S}_{C}$ y realizar la suma para todos los modos de transmisión entre los contactos considerados. Los elementos de la expresión (2.71) que nos interesan para calcular la función de transmisión son aquellos para los que $n \neq m$. Al elevar al cuadrado dichos elementos obtenemos:

$$
\left|\left(\hat{S}_{C}\right)_{n m}\right|^{2}=\frac{\hbar^{2} v_{n} v_{m}}{a^{2}} \sum_{i, j, j^{\prime}, i^{\prime}} \chi_{n}\left(q_{j}\right) G^{R}(j, i) \chi_{m}\left(p_{i}\right) \chi_{n}\left(q_{j^{\prime}}\right) G^{A}\left(i^{\prime}, j^{\prime}\right) \chi_{m}\left(p_{i^{\prime}}\right),
$$

donde de nuevo introducimos la notación de operador de Green retardado y avanzado. Se ha hecho uso de la relación entre ambos $G^{A}\left(i^{\prime}, j^{\prime}\right)=G^{R}\left(j^{\prime}, i^{\prime}\right) *$ (ecuación 2.30). Si sumamos para todos los modos correspondientes a dos contactos cualesquiera obtenemos la función de transmisión entre dichos contactos:

$$
\hat{T}_{q p}=\sum_{m \in p} \sum_{n \in q}\left|\left(\hat{S}_{C}\right)_{n m}\right|^{2}=\sum_{i, j, j^{\prime}, i^{\prime}} \Gamma_{q}\left(j^{\prime}, j\right) G^{R}(j, i) \Gamma_{p}\left(i, i^{\prime}\right) G^{A}\left(i^{\prime}, j^{\prime}\right),
$$

donde se ha definido el operador $\Gamma$ en la forma:

$$
\Gamma_{p}\left(i, i^{\prime}\right)=\sum_{m \in p} \chi_{m}\left(p_{i}\right) \frac{\hbar v_{m}}{a} \chi_{m}\left(p_{i^{\prime}}\right)
$$

Podemos obtener otra expresión para este operador si tenemos en cuenta la ecuación de autoenergías en un sistema multi-modos (2.63) y considerando, además, 
que en un sistema discreto la relación entre la velocidad y el número de onda es $\hbar v=\frac{\partial E}{\partial k}$. Si representamos entonces el operador $\Gamma$ a través de una matriz, tenemos:

$$
\hat{\Gamma}_{p}=i\left[\hat{\Sigma}_{p}^{R}-\hat{\Sigma}_{p}^{A}\right] .
$$

Usando la ecuación (2.75), la expresión (2.73) se puede escribir de forma más compacta:

$$
\hat{T}_{q p}=\operatorname{Tr}\left[\hat{\Gamma}_{\mathrm{q}} \hat{\mathrm{G}}^{\mathrm{R}} \hat{\Gamma}_{\mathrm{p}} \hat{\mathrm{G}}^{\mathrm{A}}\right] .
$$

En cuanto a la densidad local de estados local density of states(LDOS), se puede obtener a partir de la FG del sistema directamente de su definición, dada por:

$$
\operatorname{LDOS}(\mathrm{E})=-\frac{1}{\pi} \operatorname{Tr}(\operatorname{Im} \hat{\mathrm{G}}(\mathrm{E})) .
$$

En la ecuación (2.77), la traza se extiende sobre la región donde queremos calcular la densidad de estados. Si realizamos la suma sobre todos los átomos del nanotubo, obtenemos la densidad de estados total, que denotamos como DOS. La obtención de la densidad de estados en un sistema es fundamental, ya que se trata de una magnitud que permite obtener numerosas propiedades asociadas a dicho sistema, como propiedades de conducción o diversas propiedades termodinámicas. Es posible, por ejemplo, caracterizar la transición del régimen conductor al aislante, en sistemas desordenados cuasi-unidimensionales a través del estudio de las densidades parciales de estados (Ruiz et al. 2007a, Ruiz et al. 2007b).

\subsection{Método numérico}

La función de Green del nanotubo la vamos a obtener mediante la expresión (2.53). En el cálculo de dicha expresión hemos de obtener los términos de autoenergías correspondientes a los contactos $\hat{\Sigma}_{L}$ y $\hat{\Sigma}_{R}$. Consideraremos en este trabajo que 
los contactos son los propios nanotubos, que son balísticos y con los que no se producen reflexiones. De este modo se evitan algunos problemas que surgen al conectar directamente el nanotubo a dos contactos metálicos.

En el cálculo de los términos de autoenergías hemos de obtener la funciones de Green de los contactos semi-infinitos $\left(\hat{g}_{l}\right)_{L / R}$. Como de dichas funciones sólo nos interesa conocer su valor en los puntos adyacentes con el nanotubo, sólo hemos de calcular $\left(\hat{g}_{l}^{00}\right)_{L / R}$ (FG superficiales), lo que haremos utilizando el método de López Sancho et al. (1984), basado en el método de las matrices de transferencia.

La función de Green de los contactos se obtiene mediante la siguiente expresión:

$$
\left(\hat{g}_{l}^{00}\right)_{R / L}=\left(E \hat{I}-\hat{H}_{00}-\hat{H}_{01} \hat{M}\right)^{-1},
$$

donde $\hat{H}_{00}$ es el Hamiltoniano de una celda unidad del nanotubo y $\hat{H}_{01}$ es la matriz que tiene en cuenta la interacción entre dos celdas consecutivas. Para el cálculo de $\hat{T}$ utilizamos el siguiente método iterativo:

$$
\hat{M}=\hat{t}_{0}+\hat{t}_{0}^{*} \hat{t}_{1}+\hat{t}_{0}^{*} \hat{t}_{1}^{*} \hat{t}_{2}+\ldots+\hat{t}_{0}^{*} \ldots \hat{t}_{n-1}^{*} \hat{t}_{n}
$$

donde las matrices $\hat{t}, \hat{t}^{*}$ vienen dadas por las expresiones:

$$
\begin{gathered}
\hat{t}_{0}=\left(E \hat{I}-\hat{H}_{00}\right)^{-1} \hat{H}_{01}^{\dagger}, \\
\hat{t}_{0}^{*}=\left(E \hat{I}-\hat{H}_{00}\right)^{-1} \hat{H}_{01}, \\
\hat{t}_{i}=\left(\hat{I}-\hat{t}_{i-1} \hat{t}_{i-1}-\hat{t}_{i-1} \hat{t}_{i-1}\right)^{-1} \hat{t}_{i-1}^{2}, \\
\hat{t}_{i}^{*}=\left(\hat{I}-\hat{t}_{i-1} \hat{t}_{i-1}^{*}-\hat{t}_{i-1}^{*} \hat{t}_{i-1}\right)^{-1} \hat{t}_{i-1}^{* 2} .
\end{gathered}
$$


El método que acabamos de describir tiene la ventaja de que el número de iteraciones cerca de las singularidades es muy bajo en comparación con otros métodos, lo que hace que converja muy rápido. Se trata por tanto de una herramienta muy útil para el cálculo de la función de Green de los nanotubos estudiados en la presente memoria. Dicho cálculo nos permitirá obtener diversas propiedades asociadas a la transmisión electrónica, tal y como hemos descrito a lo largo de este capítulo.

\subsection{Aplicación a CNT puros e infinitos}

Como ejemplo de lo anteriormente expuesto, aplicaremos el formalismo desarrollado en el presente capítulo para la obtención de las propiedades asociadas a nanotubos de diferentes geometrías, considerando únicamente nanotubos infinitamente largos y sin defectos. Calcularemos la función de transmisión mediante la ecuación (2.76), y la densidad de estados mediante (2.77). En el siguiente capítulo

aplicaremos este formalismo al cálculo de sistemas formados mediante uniones de nanotubos de diferentes geometrías y obtendremos resultados nuevos. Vamos pues a continuación a obtener la función de transmisión y la densidad de estados para nanotubos puros con diferente geometría.

\subsubsection{Función de transmisión}

La función de transmisión $T$ la vamos a calcular para CNT puros e infinitos. Así pues, no se van a producir reflexiones en el nanotubo, por lo que el valor de la función de transmisión es un número entero para cualquier energía. Dicho valor nos informa del número de canales de transmisión para cada energía, pudiendo también obtenerse mediante las relaciones de dispersión en el nanotubo. Especialmente interesante será estudiar el valor de $T$ para la energía de Fermi $\left(E=E_{F}\right)$. Como sabemos, en un nanotubo metálico las bandas de conducción y valencia se cortan en $E=E_{F}$ y por tanto la función de transmisión es diferente de cero en todo el espectro energético. 


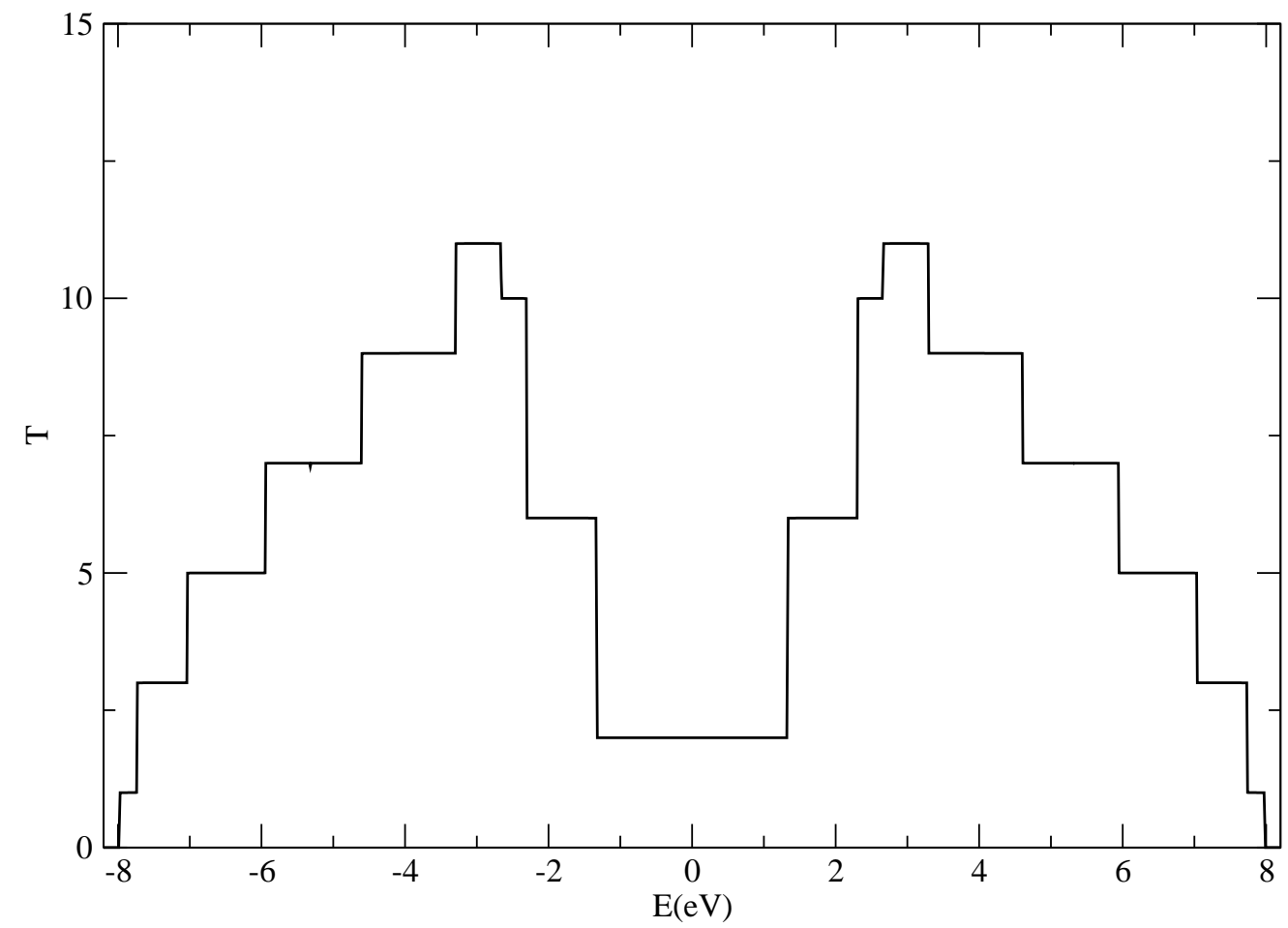

Figura 2.1: Función de transmisión para un nanotubo tipo armchair con vector quiral $(6,6)$. 
No sucede así para un nanotubo semiconductor, que presenta un gap de energía en torno a la energía de Fermi, por lo que la función de transmisión se hace cero en dicha región. El valor de dicho gap es inversamente proporcional al diámetro del nanotubo, como se ha podido comprobar experimentalmente (Iijima 1998, Wildöer et al. 1998) y viene dado por la siguiente expresión:

$$
E_{g}=\frac{|t| d_{C-C}}{d_{t}}
$$

donde $t$ es la energía de solapamiento, cuyo valor se toma igual a $-2,66 \mathrm{eV}, d_{t}$ el diámetro del nanotubo, dado por la ecuación (1.9), y $d_{C-C}$ la distancia de enlace $C-C$, igual a $1,42 \AA$.
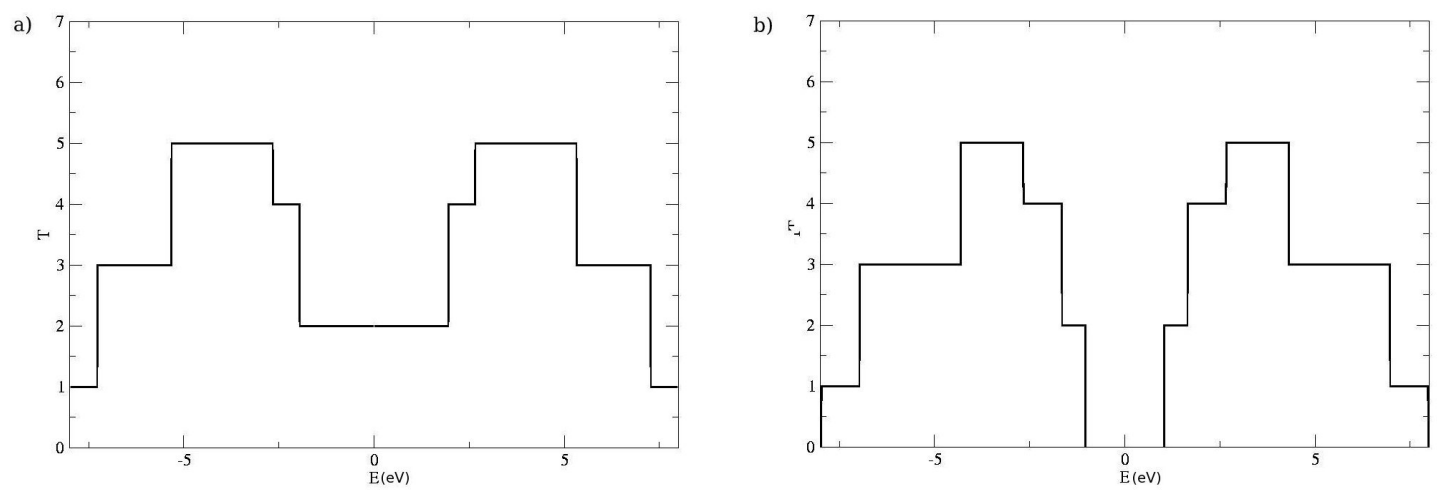

Figura 2.2: Función de transmisión para dos nanotubos tipo zigzag. a) Nanotubo metálico $(6,0)$. b) Nanotubo semiconductor $(5,0)$. La transmisión es igual a cero en este último nanotubo en la región en torno a la energía de Fermi.

Veamos diversos ejemplos para nanotubos con diferente quiralidad.

$\square$ Nanotubos tipo armchair. 
El vector quiral de un nanotubo tipo armchair se denota como $(n, n)$, presentando siempre carácter metálico. Incluimos a modo de ejemplo la función de transmisión para un nanotubo $(6,6)$ en la figura 2.1. Podemos comparar dicha figura con las relaciones de dispersion representadas en el capítulo anterior (figura 1.22). Si contamos el número de relaciones de dispersión en cada energía obtenemos el valor de la función de transmisión. Para ello hemos de tener en cuenta que 5 de las 7 curvas son doblemente degeneradas.
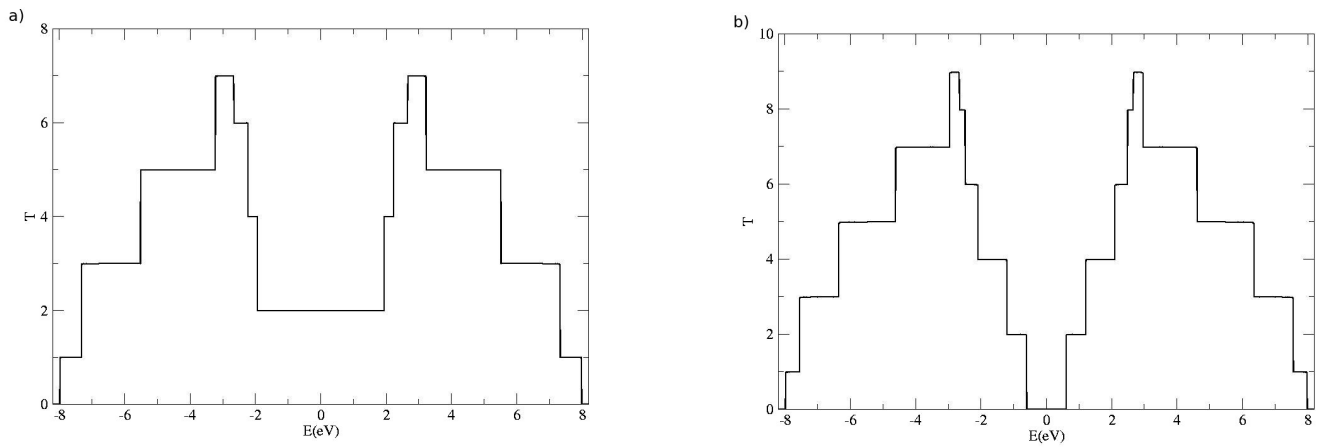

Figura 2.3: Función de transmisión para dos nanotubos tipo quiral. a) Nanotubo metálico $(5,2)$. b) Nanotubo semiconductor $(5,4)$. La transmisión es igual a cero en este último nanotubo en la región en torno a la energía de Fermi.

\section{$\square$ Nanotubos tipo zigzag.}

El vector quiral de un nanotubo tipo armchair se denota como $(n, 0)$, pudiendo presentar carácter metálico o semiconductor en función de si $n$ es o no múltiplo de tres. En la figura 2.2 mostramos la función de transmisión para dos nanotubos zigzag con diferente valor de $n$. En la figura a) se representa un CNT con $n=6$, metálico. En la figura b) se representa un CNT con $n=5$, semiconductor. Ambas representaciones pueden ser contrastadas con las relaciones de dispersión incluidas en el capítulo anterior (figura 1.23). Como vemos, el na- 
notubo $(5,0)$ presenta transmisión igual a cero en torno a la energía de Fermi. La amplitud del gap correspondiente viene dada por la expresión (2.84).

\section{$\square$ Nanotubos tipo quiral.}

Los nanotubos que no presentan geometría armchair ni zigzag se denotan como $(n, m)$, pudiendo presentar carácter metálico o semiconductor en función de si la diferencia $n-m$ es o no múltiplo de tres. Ambos casos se representan mediante un ejemplo en la figura 2.3, donde se muestra la función de transmisión para un CNT $(5,2)$ metálico, y para un CNT $(5,4)$, con carácter semiconductor.

\subsubsection{Densidad de estados}

Presentamos en este subapartado algunos ejemplos de la densidad de estados total (DOS) para algunos de los sistemas estudiados en el subapartado anterior. Especialmente interesante resulta el análisis del comportamiento de esta función en la región en torno al nivel de Fermi, que reproduce las mismas características ya estudiadas en el apartado 2.5.1 para la función de transmisión. De igual interés resultan las singularidades que aparecen en los máximos y mínimos de las relaciones de dispersión, como veremos a continuación.

\section{$\square$ Nanotubos metálicos.}

En la figura 2.4 se representa la densidad de estados total en función de la energía para un nanotubo tipo armchair $(6,6)$ que, como sabemos, presenta carácter metálico. Puede observarse que la densidad de estados no se hace cero en ninguna región del espectro energético. Las singularidades que presenta la figura aparecen para energías que se corresponden con máximos o mínimos relativos en las relaciones de dispersión. Este tipo de resonancias, que reciben el nombre de singularidades de Van-Hove, se han detectado también de forma 


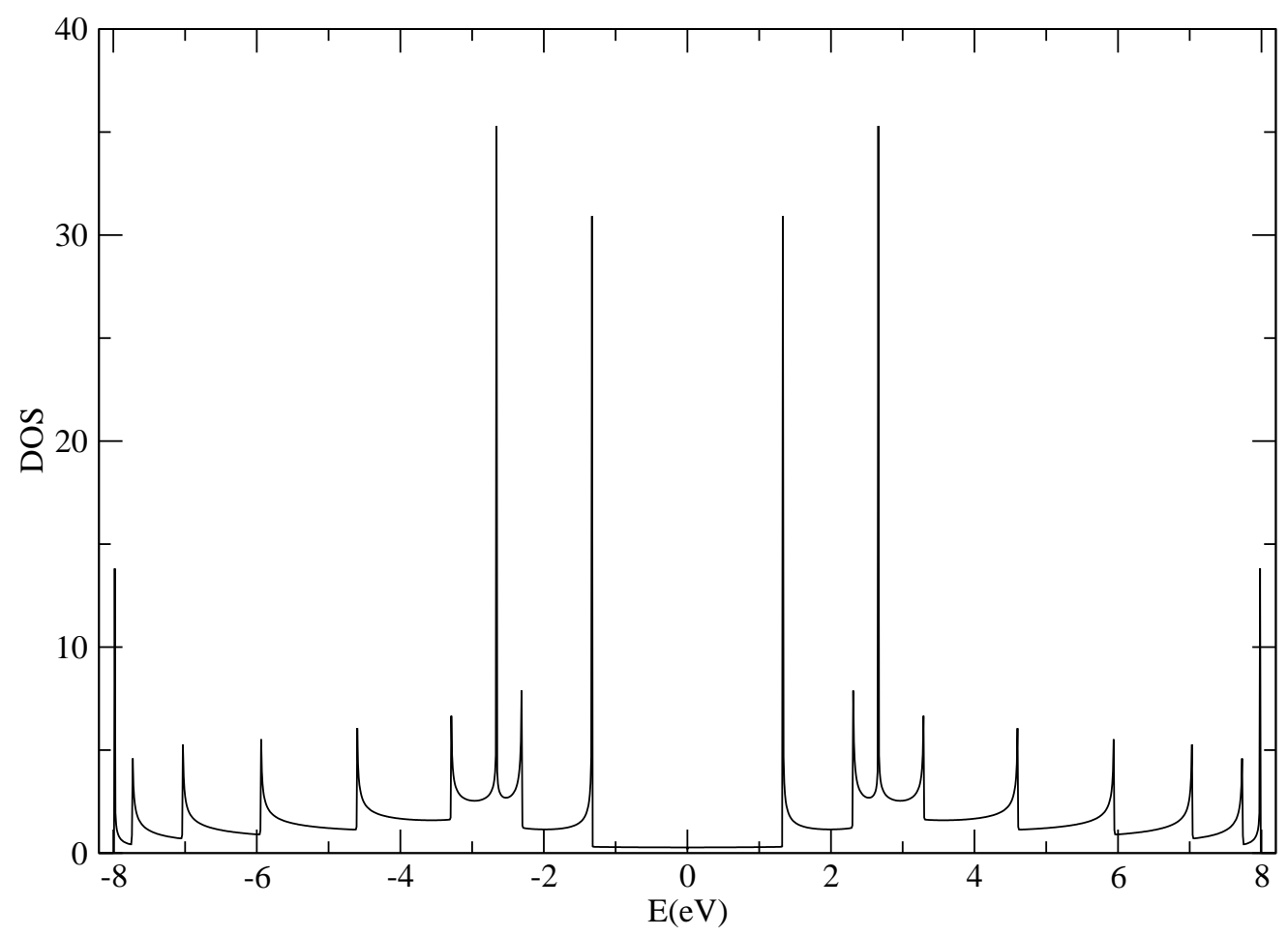

Figura 2.4: Densidad de estados para un nanotubo tipo armchair con vector quiral $(6,6)$, de carácter metálico. Se aprecia claramente que la densidad de estados es distinta de cero para todo el rango de energías. Las singularidades de Van-Hove se ponen de manifiesto en los máximos y mínimos relativos de las relaciones de dispersión. 
experimental (Wildöer et al. 1998). Estas singularidades aparecen debido a que, en general, la densidad de estados de un sistema la podemos obtener como una integral de la inversa de $\Delta_{k} E(k)$, donde $\Delta_{k}$ es el gradiente respecto de $k$ y $E(k)$ es la relación de dispersión. En los puntos donde $\Delta_{k} E(k)=0$ tenemos singularidades integrables, esto es, dos valores finitos para la densidad de estados pero dos divergencias en la pendiente, $\frac{d(D O S)}{d E}$.

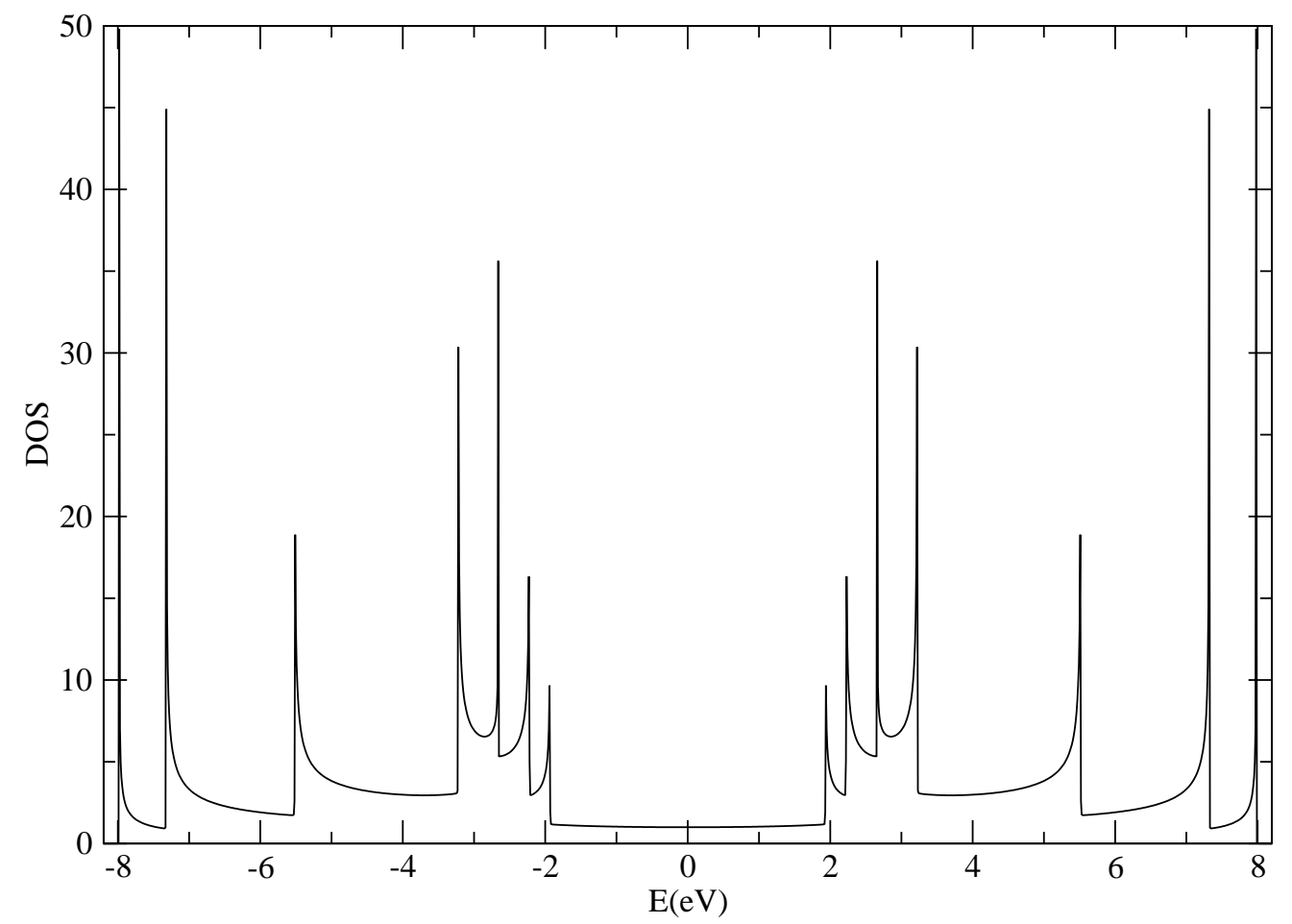

Figura 2.5: Densidad de estados para un nanotubo con geometría quiral $(5,2)$. Se trata de un nanotubo metálico, por lo que la densidad de estados es distinta de cero en todo el espectro.

En la figura 2.5 se representa la densidad de estados frente a la energía para un 
nanotubo $(5,2)$, que presenta también carácter metálico pero cuya geometría es de tipo quiral. El comportamiento de la densidad de estados en este caso es muy similar al del nanotubo tipo armchair representado en la figura 2.4. Al ser también un nanotubo metálico, la densidad de estados es distinta de cero para todo el espectro. Aparecen, así mismo, las singularidades de Van-Hove para las energías que se corresponden con máximos y mínimos relativos en las relaciones de dispersión.

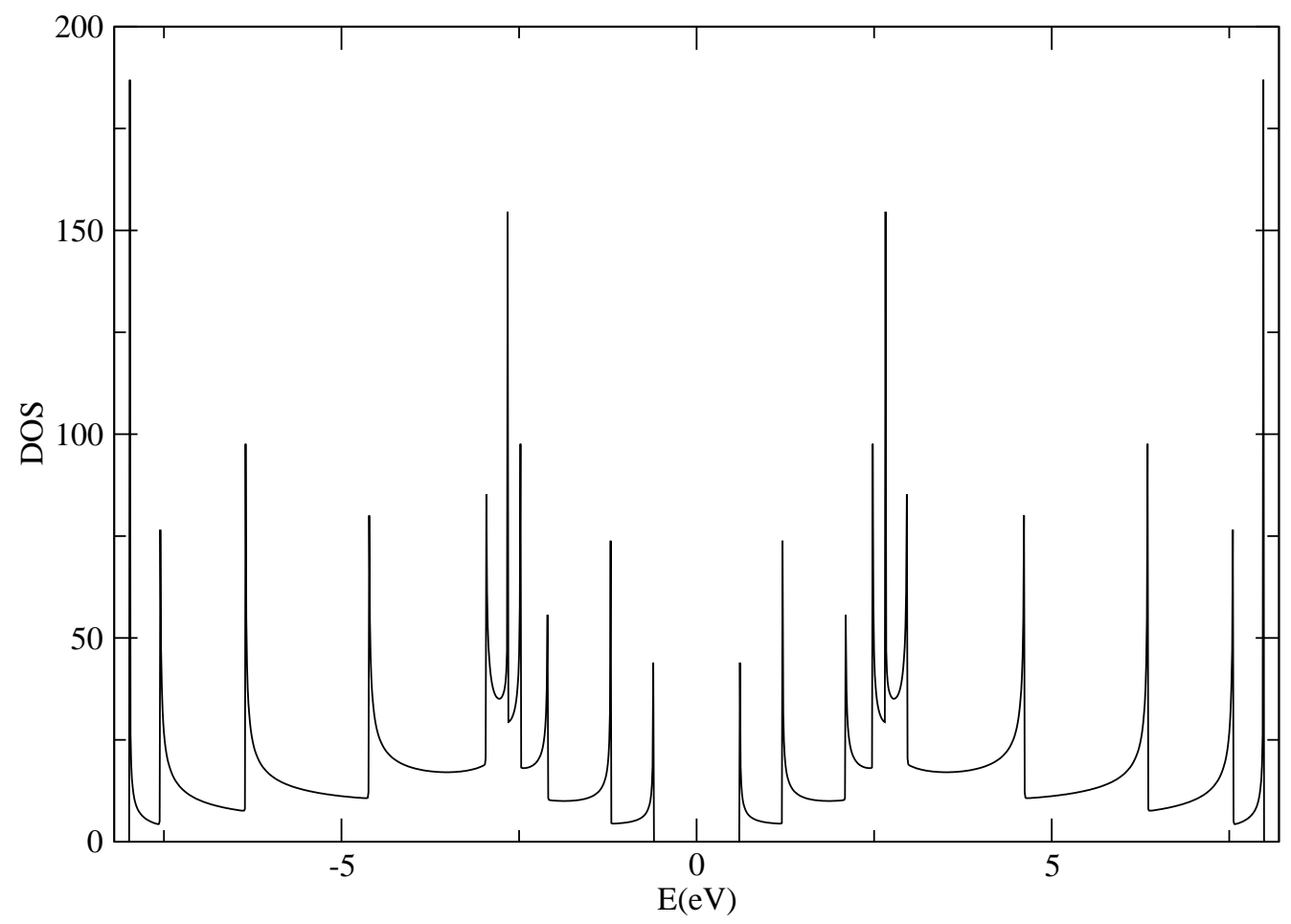

Figura 2.6: Densidad de estados para un nanotubo con geometría quiral $(5,4)$. Se trata de un nanotubo semiconductor. Puede verse claramente en la figura que la densidad de estados se hace cero en la región en torno a la energía de Fermi. 


\section{$\square$ Nanotubos semiconductores.}

Como ya hemos estudiado en los apartados anteriores, los nanotubos semiconductores presentan un gap en la conducción en torno a la energía de Fermi. Hemos visto algunos ejemplos en el subapartado 2.5.1, donde la función de transmisión se hacía igual a cero en dicha región. La densidad de estados muestra este mismo comportamiento en nanotubos semiconductores, como podemos comprobar en la figura 2.6. Si nos fijamos nuevamente en la región en torno a la energía de Fermi, vemos cómo en el CNT $(5,4)$ no hay estados en dicha región. Región que se corresponde con aquella en que la transmisión electrónica del sistema es igual a cero. En los valores de energía que se corres-

ponden con extremos de las relaciones de dispersión se aprecian claramente las singularidades de Van-Hove. 


\section{Capítulo 3}

\section{Estudio de Cavidades de CNT}

Una vez expuestos los fundamentos teóricos en los que se basa este trabajo, nos proponemos realizar el estudio de las propiedades de transporte electrónico en diferentes sistemas compuestos por nanotubos de carbono. En el presente capítulo vamos a trabajar con cavidades de CNT. Llamamos así a las estructuras formadas mediante la unión de nanotubos de diferente diámetro, lo que da lugar a que el sistema presente ensanchamientos y estrechamientos a lo largo del mismo. Nos centraremos principalmente en las cavidades formadas por nanotubos de tipo armchair, que presentan carácter metálico, aunque también realizaremos el estudio en cavidades de nanotubos semiconductores.

Comenzaremos por obtener las propiedades asociadas a la conducción electrónica en una sóla cavidad (Jódar et al. 2006). Completaremos nuestro estudio mediante el análisis de la dependencia de dichas propiedades con la geometría del sistema. En concreto, analizaremos su comportamiento en función de la anchura de la cavidad. Así mismo, realizaremos el acoplamiento de dos o más cavidades, para finalmente obtener las propiedades en el límite de infinitas cavidades. 


\subsection{Descripción de las cavidades de nanotubos}

Como ya hemos mencionado, en este capítulo vamos a estudiar las propiedades de estructuras formadas por la unión entre nanotubos de diferente geometría. Supongamos un nanotubo central unido a otros dos nanotubos diferentes por ambos ex-

tremos. Este tipo de estructuras las denotamos como $\left(n_{1}, m_{1}\right) / N\left(n_{2}, m_{2}\right) /\left(n_{3}, m_{3}\right)$, donde $\left(n_{1}, m_{1}\right)$ y $\left(n_{3}, m_{3}\right)$ son nanotubos semi-infinitos usados como contactos, y $\left(n_{2}, m_{2}\right)$ es el nanotubo central que posee $N$ celdas unidad. Nosotros trabajaremos principalmente con estructuras formadas mediante nanotubos tipo armchair, que como sabemos presentan carácter metálico. Utilizamos como contactos nanotubos con el mismo vector quiral. Así pues, denotaremos esta estructura como $(n, n) / N(m, m) /(n, n)$, donde $n \neq m$. Se pueden presentar dos casos diferentes:

$\square n>m$. El nanotubo central posee un menor número de canales de transmisión que los nanotubos de los extremos, de acuerdo con la ecuación (1.29). Así mismo, el diámetro del nanotubo central es menor que el de los extremos, ecuación (1.9). Tenemos pues un estrechamiento en la parte central de la estructura.

$\square n<m$. En este caso existe un mayor número de canales de transmisión en la parte central de la estructura. Además, ésta presenta un diámetro mayor que el de los extremos. De esta manera, se forma un ensanchamiento de la estructura en la región central, a la que llamaremos cavidad.

Otros autores han realizado diferentes estudios con otro tipo de estructuras en las que las uniones entre nanotubos provocan la presencia de un gap en la conductancia debido a los cambios en las simetrías rotacionales. Estos cambios no se producen en la unión de nanotubos tipo armchair. En este caso los autores encontraron que el sistema muestra un comportamiento que se asemeja al de un punto cuántico (Chico y Jaskolski 2004). Se han estudiado así mismo nanotubos con es- 
trechamientos estructurales por deformación mecánica del nanotubo, que producen resonancias en la transmisión (Sim et al. 2001).

En la figura 3.1 se muestra una cavidad del tipo $(6,6) / 4(9,9) /(6,6)$, que es la que utilizaremos en primer lugar en nuestros cálculos. Existen dos regiones de transición entre el nanotubo central y los dos extremos, que llamaremos uniones. En dichas uniones es necesario insertar cierto número de defectos para que el acoplamiento sea posible. Nosotros hemos insertado un par de defectos en cada unión: un pentágono y un heptágono, señalados en la figura en color rojo.
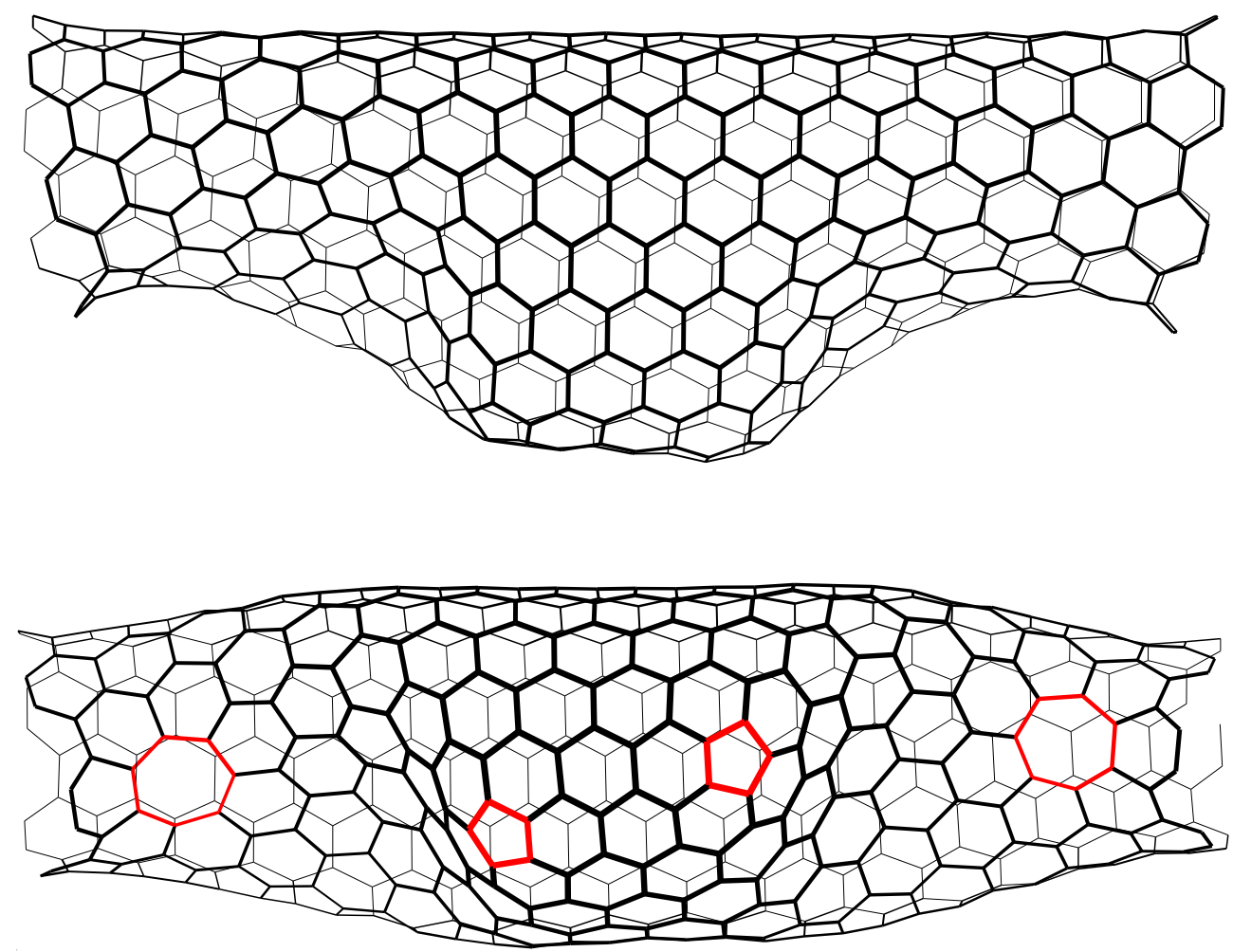

Figura 3.1: Dos vistas diferentes de la estructura $(6,6) / 4(9,9) /(6,6)$. En la parte inferior se aprecian, en color rojo, los defectos producidos en la unión de los diferentes nanotubos (un pentágono y un heptágono en cada unión). 


\subsection{Estudio de la cavidad $(6,6) / 4(9,9) /(6,6)$}

En este apartado vamos a estudiar las propiedades de un tipo particular de cavidad formada mediante nanotubos de tipo armchair. Acoplaremos cuatro celdas del nanotubo $(9,9)$ por ambos extremos a dos nanotubos $(6,6)$, para formar así la cavidad $(6,6) / 4(9,9) /(6,6)$. Calcularemos la función de transmisión, la densidad local de estados, y las autofunciones y autoenergías, lo que nos permitirá obtener las funciones de participación correspondientes.

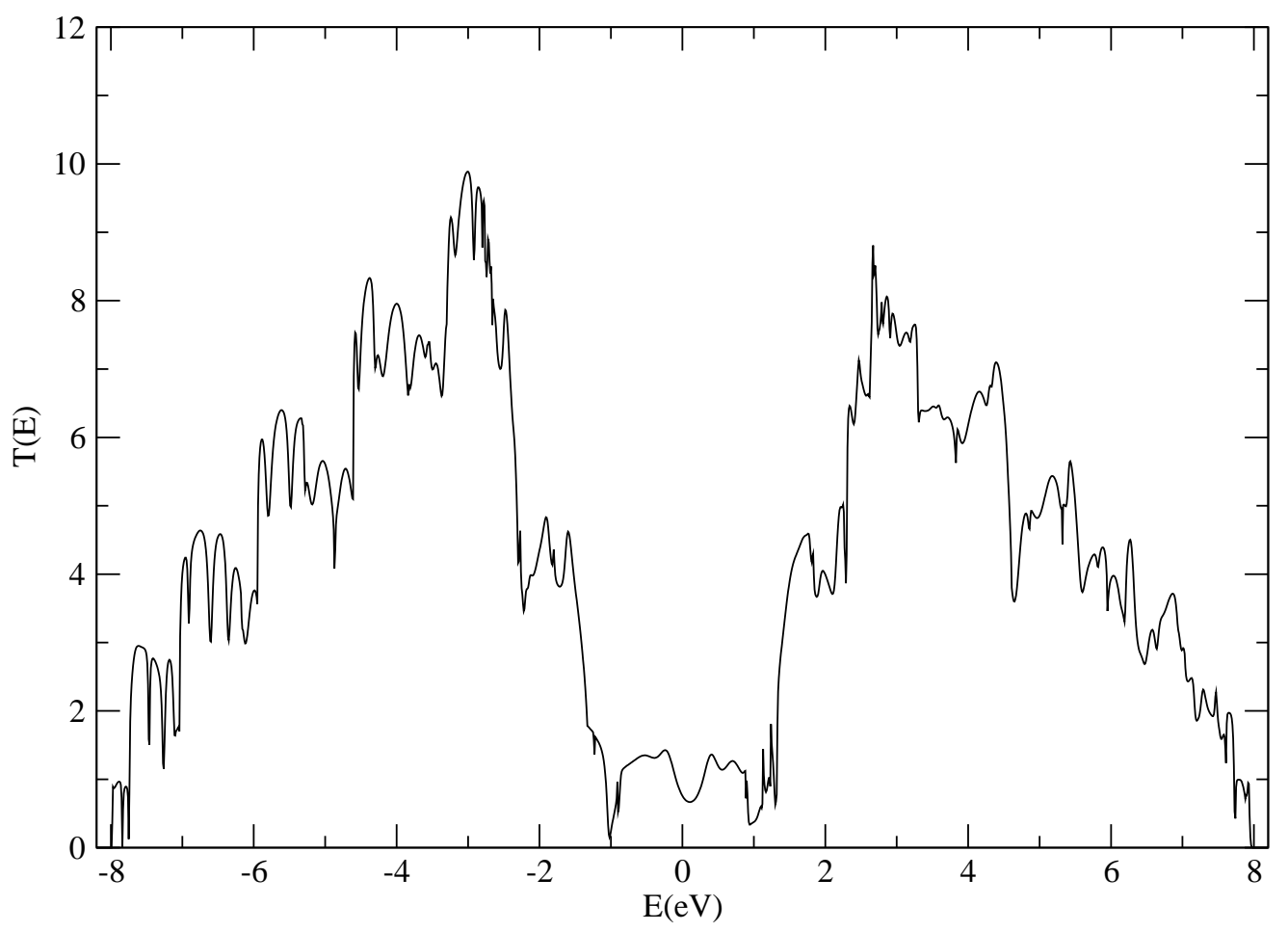

Figura 3.2: Función de transmisión de la cavidad $(6,6) / 4(9,9) /(6,6)$, donde se aprecian claramente dips en dicha función. 


\subsubsection{Función de transmisión}

La función de transmisión de nuestro sistema la calculamos mediante la expresión (2.76). La presencia de la región de transición en la unión de los nanotubos hace que se produzcan reflexiones en dicha unión, a diferencia de lo que sucede en los nanotubos puros, como vimos en el apartado 2.5.1. Así, la función de transmisión deja de estar cuantizada, pudiendo tomar un valor no entero. Por el mismo motivo se produce una asimetría de las bandas de energía en torno al nivel de Fermi, por lo que tanto la transmisión como la densidad de estados dejan de ser funciones simétricas respecto a la energía de Fermi (Tamura y Tsukada 1994). En la figura 3.2 se muestra la función de transmisión de la cavidad $(6,6) / 4(9,9) /(6,6)$ en función de la energía.

Llaman la atención especialmente en esta figura las disminuciones bruscas en la transmisión, que vamos a llamar dips, y que aparecen para ciertas energías. Como demostraremos, este hecho está relacionado con la existencia de estados cuasilocalizados en el interior de la cavidad.

Para explicar el comportamiento de la función de transmisión comparamos dicha función con las correspondientes de los nanotubos puros, $(6,6)$ y $(9,9)$, que se muestran con diferentes colores en la figura 3.3. Como vemos en dicha figura, el número de canales de transmisión para el nanotubo $(6,6)$ es menor o igual al del $(9,9)$. Por lo tanto, en el acoplamiento de ambos la transmisión queda limitada por el número de canales del $(6,6)$. En torno a la energía de Fermi, donde éste número para ambos nanotubos es igual a dos, se aprecian oscilaciones pero no dips en la transmisión. Esto significa que la causa de que se produzcan dichos dips puede estar relacionada con la diferencia de canales entre los dos nanotubos. Si llamamos $\Delta T$ a la diferencia entre las funciones de transmisión de ambos nanotubos, $\Delta T(E)=T_{(9,9)}(E)-T_{(6,6)}(E)$, y la comparamos con $T$ de la cavidad podemos tener una visión más clara de la relación entre ambas (figura 3.4). 


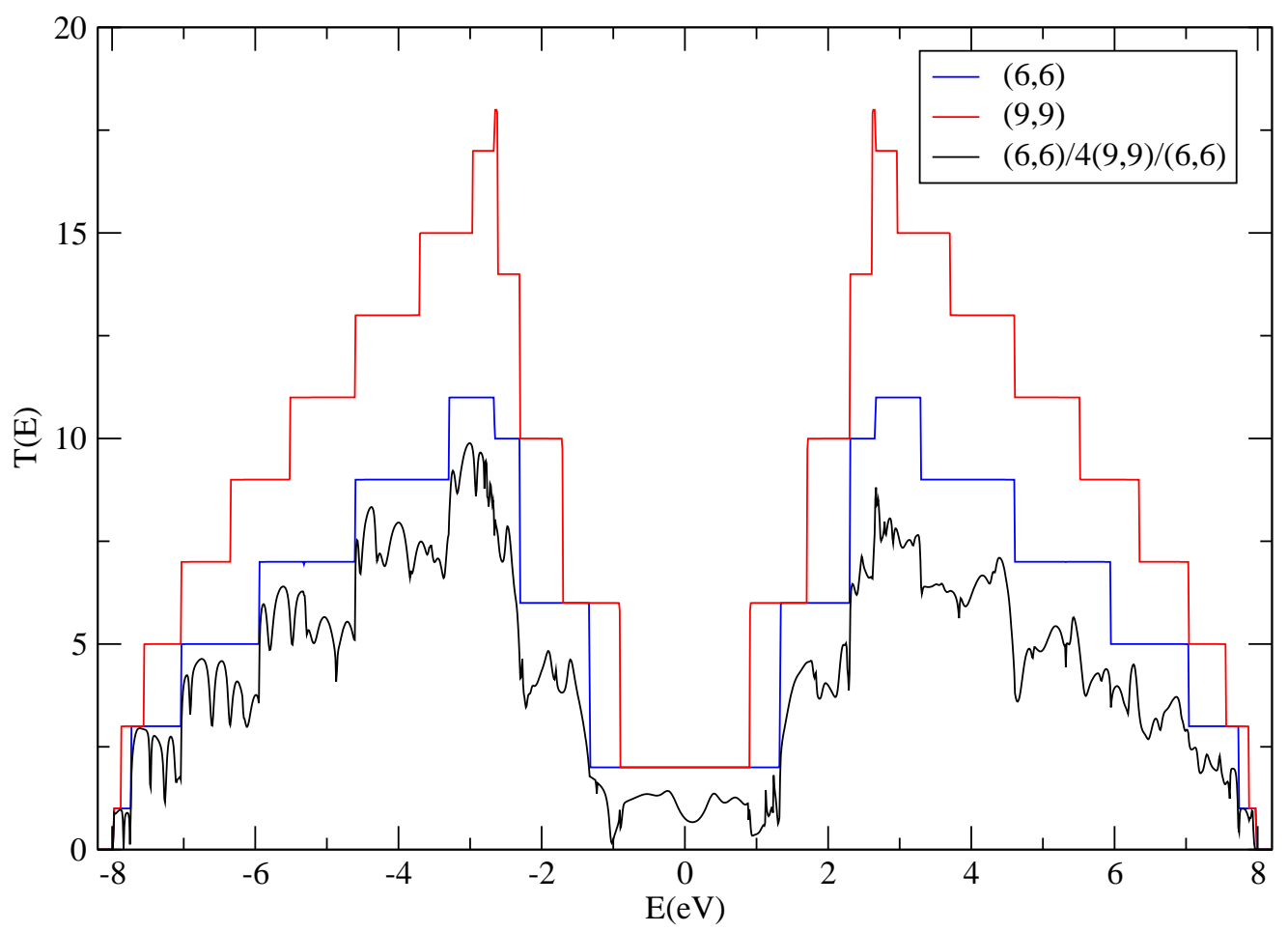

Figura 3.3: Función de transmisión del nanotubo $(6,6)$ (azul), $(9,9)$ (rojo) y estructura $(6,6) / 4(9,9) /(6,6)$ (negro). En la región central en la función de transmisión de la cavidad, donde coinciden el número de canales de los nanotubos $(6,6)$ y $(9,9)$, no se producen anti-resonancias. 

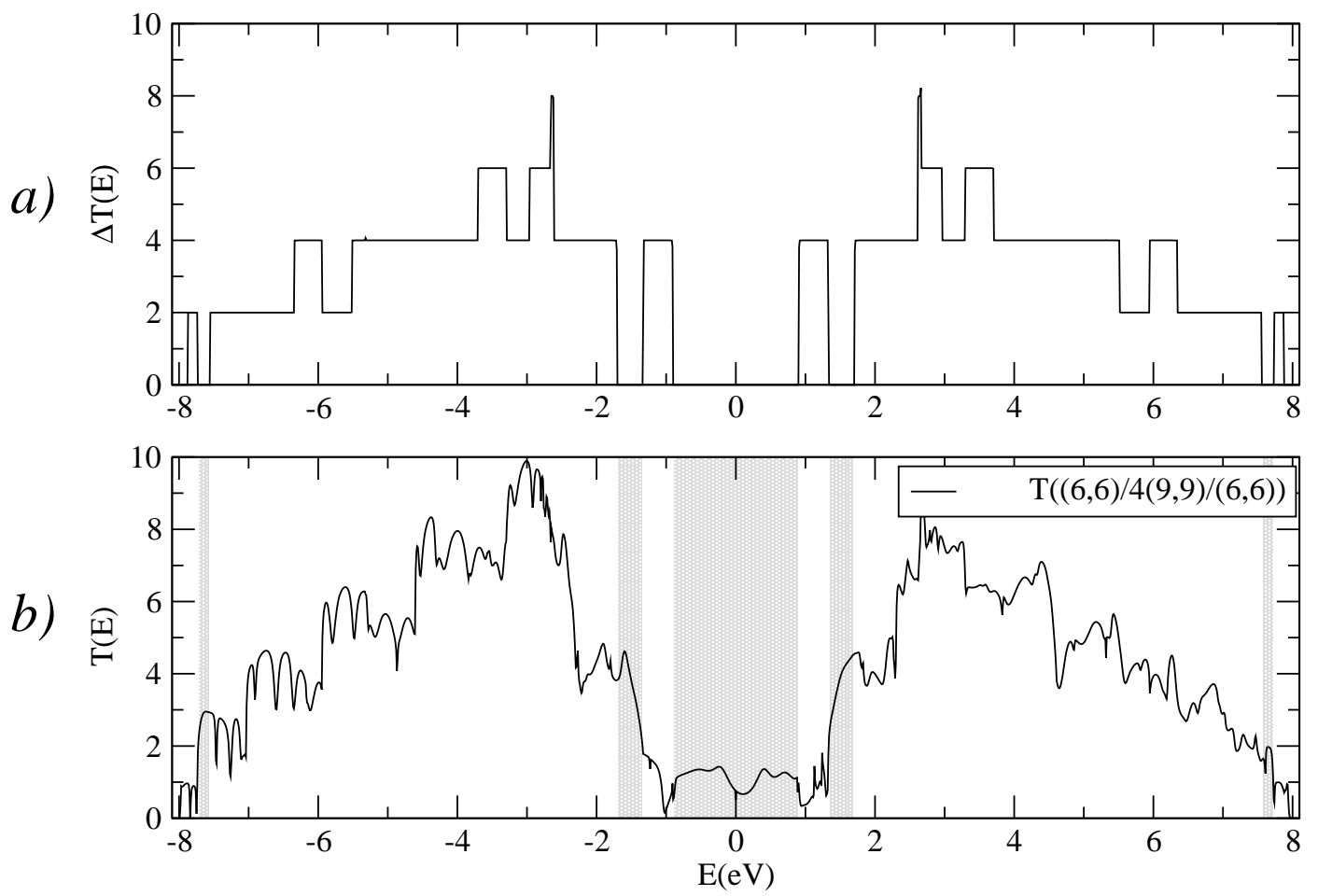

Figura 3.4: a) Diferencia entre la transmisión de los nanotubos $(9,9)$ y $(6,6), \Delta T(E)=T_{(9,9)}(E)-T_{(6,6)}(E)$. b) Función de transmisión para la cavidad $(6,6) / 4(9,9) /(6,6)$. Las regiones sombreadas son aquellas en las que $\Delta T(E)=0$. En dichas regiones no existen dips en la transmisión. 
Como se puede observar en la figura 3.4, no existen dips en la transmisión en las regiones sombreadas, que son aquellas en las que el número de canales en los dos nanotubos utilizados en la cavidad es el mismo, es decir, $\Delta T(E)=0$. Sí pueden apreciarse oscilaciones en las regiones sombreadas, que se deben a las múltiples reflexiones que se producen en las uniones de los dos nanotubos. La superposición de las ondas reflejadas en dichas uniones genera las resonancias que se aprecian en la figura $3.4 \mathrm{~b}$ ). Fijémonos ahora en las regiones $0,91 \mathrm{eV}<|E|<1,33 \mathrm{eV}$. En estas dos regiones se aprecia un fuerte dip en la transmisión. En la figura 3.3 podemos comprobar que el CNT $(6,6)$ posee en dicha región dos canales de transmisión, mientras que el CNT $(9,9)$ posee cuatro. Así pues, existen dos canales que se extienden a lo largo de toda la estructura, mientras que otros dos están localizados únicamente en la cavidad. Esto hace que se produzcan interferencias destructivas entre los estados localizados y extendidos en la cavidad, que dan lugar a estas anti-resonancias. El resultado es similar al que encontraron Sim et al. (2001) para nanotubos con estrechamientos estructurales por deformación mecánica del nanotubo.

Las resonancias producidas en el rango de energías $-0,91 \mathrm{eV}<|E|<0,91 \mathrm{eV}$ deben mostrar cierta dependencia con la longitud de la cavidad, $N$, ya que como hemos mencionado, dichas resonancias se deben a interferencias entre las ondas reflejadas en las uniones de la cavidad. Para comprobarlo, calculamos la función de transmisión para estructuras con diferente valor de $N$. En la figura 3.5 mostramos los resultados para tres cavidades del tipo $(6,6) / N(9,9) /(6,6)$ con $N=2,4$ y 8 . Como se aprecia en dicha figura, las resonancias muestran una fuerte dependencia con la longitud de la cavidad. Cuanto mayor es el valor de $N$, las resonancias son menos pronunciadas pero a la vez aumenta el número de las mismas. Esto se debe a que al aumentar el tamaño aumenta el número de estados resonantes en la cavidad, por lo que aumenta también la probabilidad de tener efecto túnel por resonancia (resonant tunneling). 


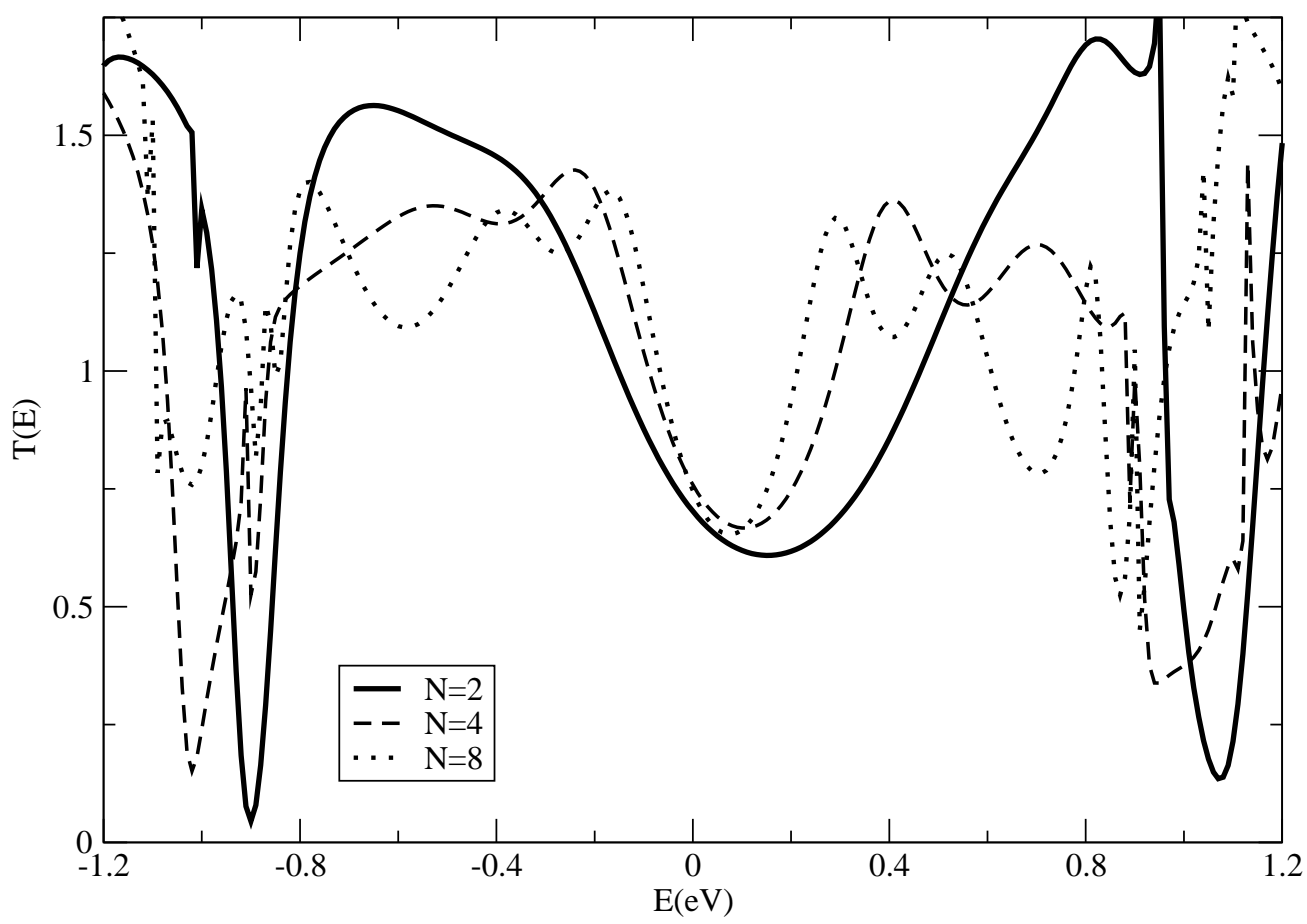

Figura 3.5: Función de transmisión en función de la energía para tres cavidades del tipo $(6,6) / N(9,9) /(6,6)$, en el rango de energías $-1,2 \mathrm{eV}<E<1,2 \mathrm{eV}$. La línea continua muestra el resultado obtenido para $N=2$. La línea discontinua para $N=4$, y la línea de puntos para $N=8$. Se aprecia una fuerte dependencia de la intensidad y número de resonancias con la longitud de la cavidad. 


\subsubsection{Densidad de estados}

En el apartado 3.2.1 adelantamos que la presencia de los dips en la transmisión para la estructura $(6,6) / 4(9,9) /(6,6)$ se relaciona con la aparición de estados cuasilocalizados dentro de la cavidad. Para comprobar esta afirmación, podemos recurrir al cálculo de la densidad local de estados, que realizaremos a través de la expresión (2.77). Mediante esta expresión podemos calcular LDOS en cualquier región del nanotubo sin más que extender la traza que aparece en la ecuación (2.77) sobre los átomos pertenecientes a dicha región.

Obtendremos la densidad local de estados para dos regiones: en el interior de la cavidad y en los contactos. Comparando los resultados obtenidos para ambas densidades podemos comprobar la existencia de estados cuasi-localizados en la cavidad. Dichos estados se corresponden con picos de la densidad local de la cavidad para ciertas energías que no están presentes en la densidad local de estados en los contactos.

Los resultados obtenidos se muestran en la figura 3.6, en unidades arbitrarias. En dicha figura se aprecian claramente dicrepancias entre los picos de ambas densidades locales de estados. Existen numerosos estados en la cavidad que no se corresponden con estados fuera de la misma. Estos estados están relacionados con ondas cuasilocalizadas. Luego confirmaremos esto mediante el uso del radio de participación.

Veamos ahora la relación entre los picos de la densidad local de estados en la cavidad y la función de transmisión. En la figura 3.7 se muestran ambas funciones, en unidades arbitrarias. Puede verse claramente que los dips en la transmisión se corresponden con los picos de LDOS en la cavidad, lo cual efectivamente demuestra que existe una relación entre ambos, y podemos por tanto afirmar que la caída en la transmisión para ciertas energías se debe a la presencia de estados cuasi-localizados en el interior de la cavidad. 


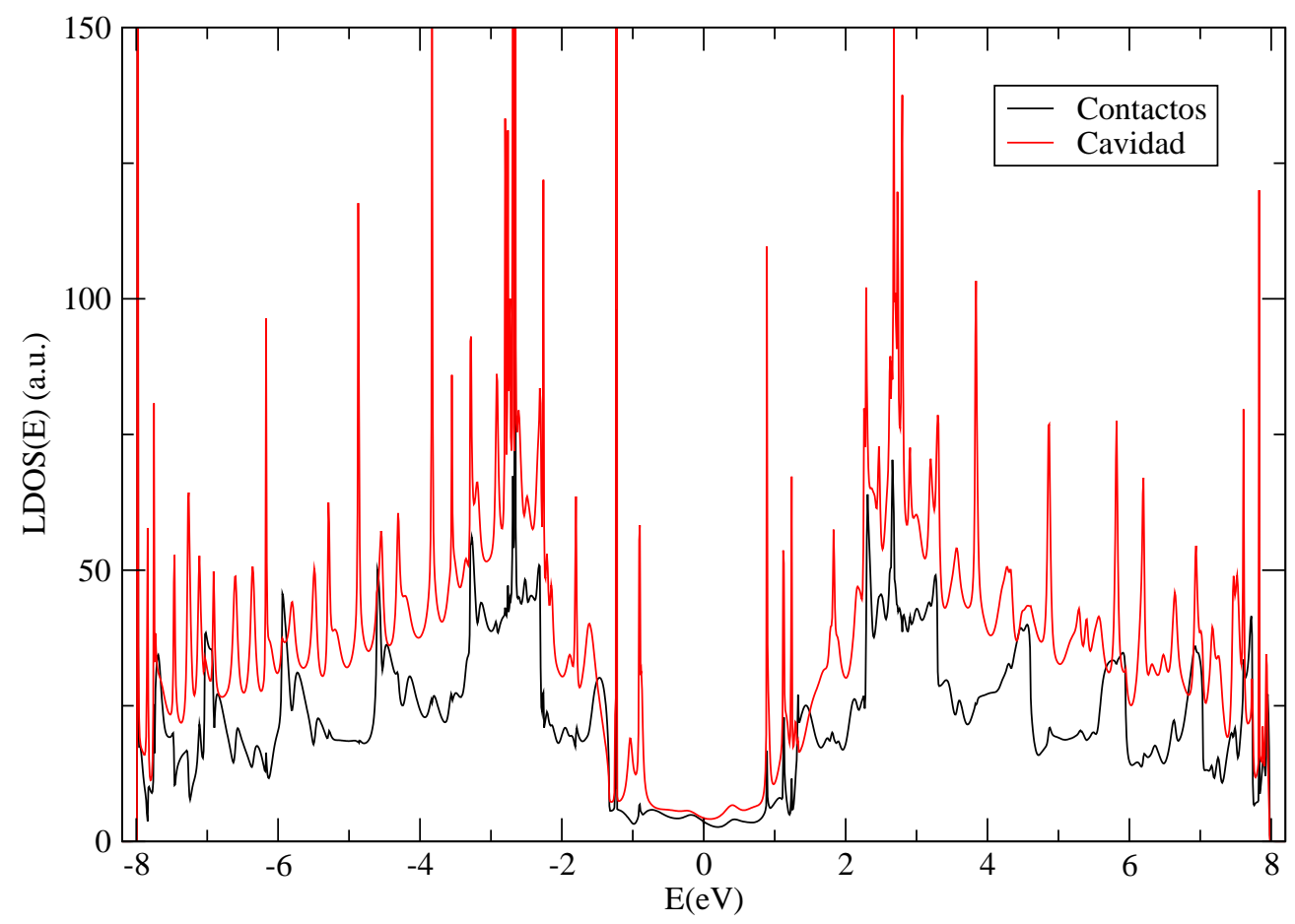

Figura 3.6: Densidades locales de estados para la estructura $(6,6) / 4(9,9) /(6,6)$. La curva en color negro se corresponde con LDOS en los contactos. La curva en color rojo representa LDOS en la cavidad, que muestra numerosos picos que no existen en la densidad local de estados de los contactos. 


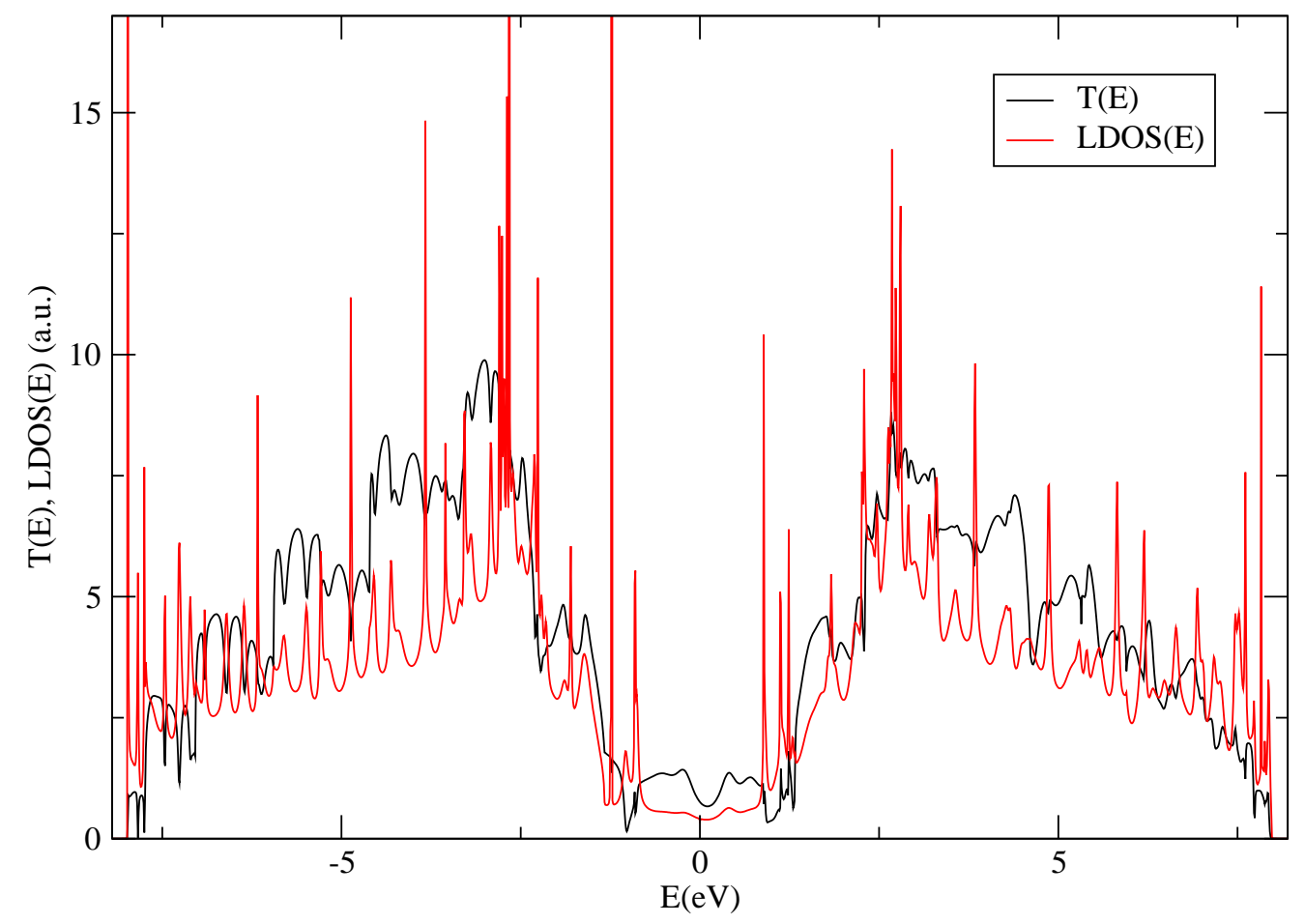

Figura 3.7: Función de transmisión para la estructura $(6,6) / 4(9,9) /(6,6)$ (curva en color negro) y densidad local de estados en la cavidad (curva en color rojo). Vemos claramente que los picos de LDOS se corresponden con los dips en la transmisión. 


\subsubsection{Función de participación y funciones de onda}

Otra forma de corroborar nuestra afirmación de que la presencia de estados cuasi-localizados en la cavidad provoca la aparición de dips en la transmisión de nuestra estructura, es calcular las funciones de onda y medir su radio o función de participación. En el apartado 2.1.1 explicamos que la función de participación es una medida del número de átomos sobre los que se extiende la función de onda. Así pues, es una herramienta muy útil para distinguir los estados localizados de los extendidos.

La función de participación se calcula a través de la expresión (2.16), donde previamente hemos de calcular las autofunciones y autoenergías para la estructura $(6,6) / 4(9,9) /(6,6)$. Para este cálculo no vamos a usar el método recursivo que nos permitía extender los contactos para hacerlos semi-infinitos. Vamos, en cambio, a trabajar con nanotubos finitos y para minimizar los efectos de los bordes de nuestra estructura, extendemos los contactos haciendo que la estructura final sea lo suficientemente larga. En concreto, la alargaremos hasta un total de 1920 átomos, suficiente para los cálculos que pretendemos realizar.

En la figura 3.8 se representa la función de participación para todas las autofunciones obtenidas en función de la energía. La línea horizontal discontinua se corresponde con un valor $P_{n}=600$. Vamos a considerar que, para el nanotubo empleado, las ondas con un valor de la función de participación menor de 600 son cuasi-localizadas, ya que se extienden sobre menos de la tercera parte de los átomos totales. Las zonas sombreadas se corresponden con energías para las cuales el número de canales de los nanotubos $(6,6)$ y $(9,9)$ es el mismo. Como vemos en la figura, apenas existen estados cuasi-localizados para estas energías, como esperábamos al afirmar que la presencia de estados localizados se relaciona con la diferencia de canales entre los contactos y la cavidad. 


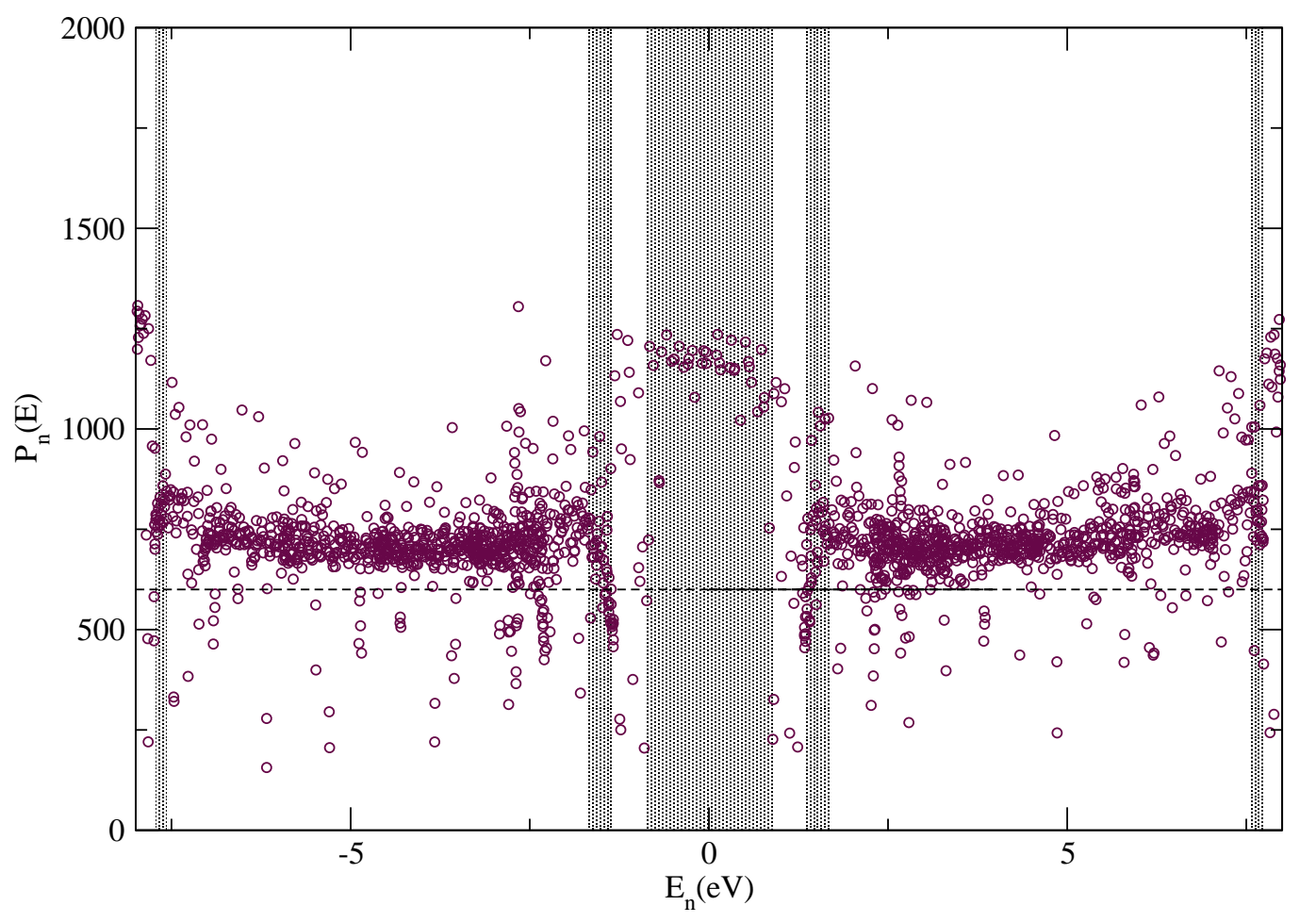

Figura 3.8: Función de participación para la estructura $(6,6) / 4(9,9) /(6,6)$, formada mediante 1920 átomos, en función de la energía. La línea horizontal discontinua separa los estados con $P_{n}<600$, que pueden considerarse cuasi-localizados, de los estados con $P_{n}>600$. Las regiones sombreadas se corresponden con energías para las cuales $\Delta T=0$. En dichas regiones no se encuentran apenas estados cuasi-localizados. 


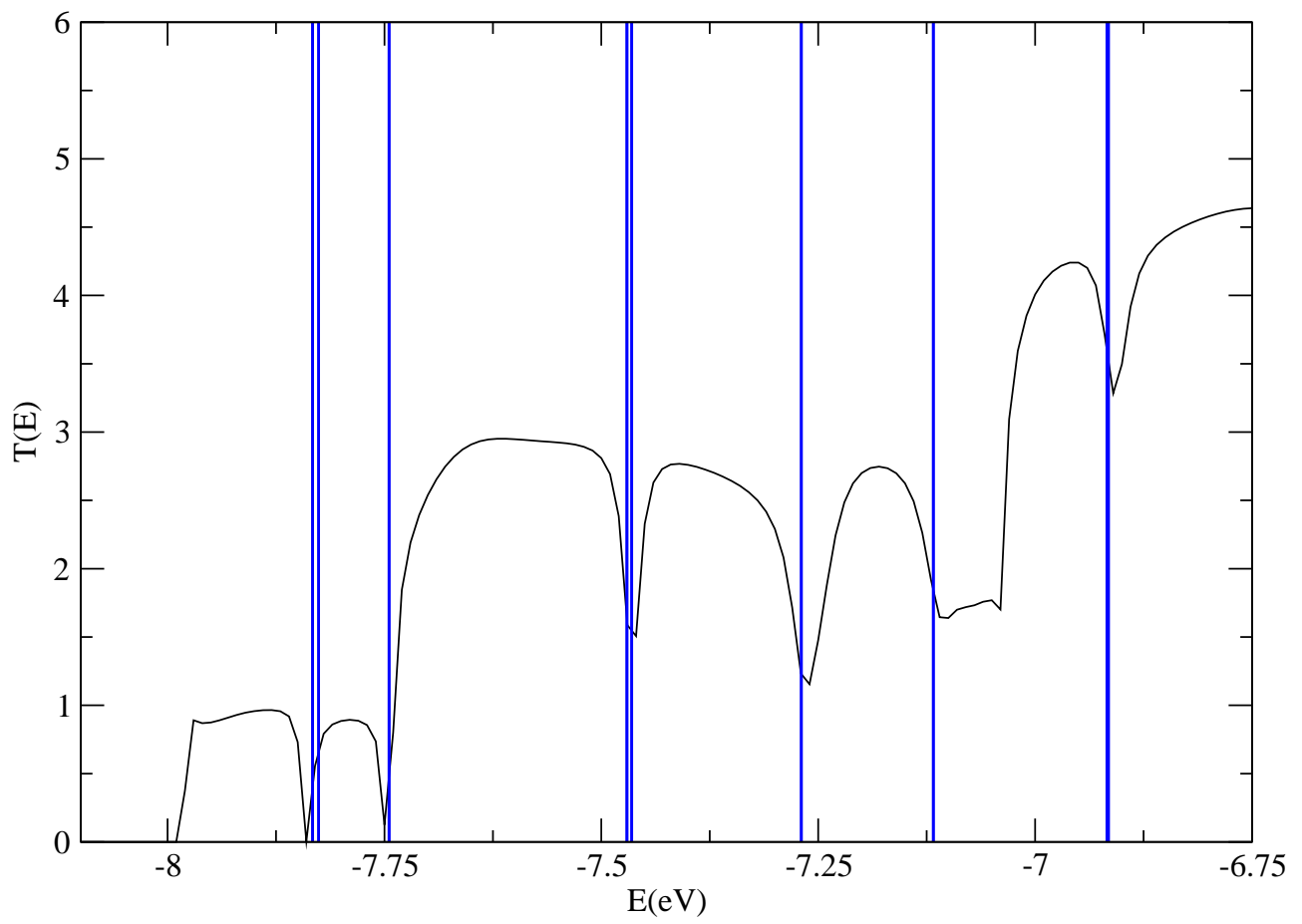

Figura 3.9: Función de transmisión de la estructura $(6,6) / 4(9,9) /(6,6)$. Por claridad, sólo mostramos las energías inferiores a $-6,75$ eV. Las líneas verticales muestran los estados con función de participación menor de 600 en dicho rango de energías. Existe una clara correspondencia entre los dips en la transmisión y los estados cuasi-localizados. 
Debemos ahora comprobar si las autoenergías de las ondas cuasi-localizadas se corresponden con las energías de los dips en la transmisión. Para ello, inspeccionamos la función de transmisión junto con líneas verticales trazadas en energías donde existen ondas cuasi-localizadas. Comprobamos así que hay una gran coincidencia de los dips con estas líneas verticales. Como ejemplo, mostramos la figura 3.9, donde se representa la función de transmisión para energías menores de $-6,75 \mathrm{eV}$. Como vemos, existe una clara correspondencia entre dichos estados y los dips en la transmisión. El pequeño desplazamiento en energía que se aprecia entre las líneas verticales y los dips es debido a los efectos de tamaño finito. Esto es así porque la función de transmisión se calcula usando el método de la función de Green para un sistema infinito, mientras que en el cálculo de la función de participación hemos usado un nanotubo finito. La figura 3.9 nos confirma de nuevo que las ondas cuasilocalizadas que se encuentran en el interior de la cavidad son las que provocan las anti-resonancias en la transmisión.

Para terminar este apartado, mostramos en la figura 3.10 la forma de tres de las autofunciones cuasi-localizadas de la figura 3.9, a modo de ejemplo. Se representa en dicha figura la probabilidad de ocupación de las autofunciones en función del índice $n$ que etiqueta a los átomos de la estructura $(6,6) / 4(9,9) /(6,6)$. Como vemos la mayor probabilidad de ocupación de estas tres autofunciones se encuentra sobre la misma región: la parte central del nanotubo. Además, otras funciones de onda localizadas aparecen en los extremos del nanotubo finito. No las mostramos debido a que éstas sólo aparecen en el nanotubo finito empleado para calcular $P_{n}$ y no existen en el infinito usado al obtener la función de transmisión. 


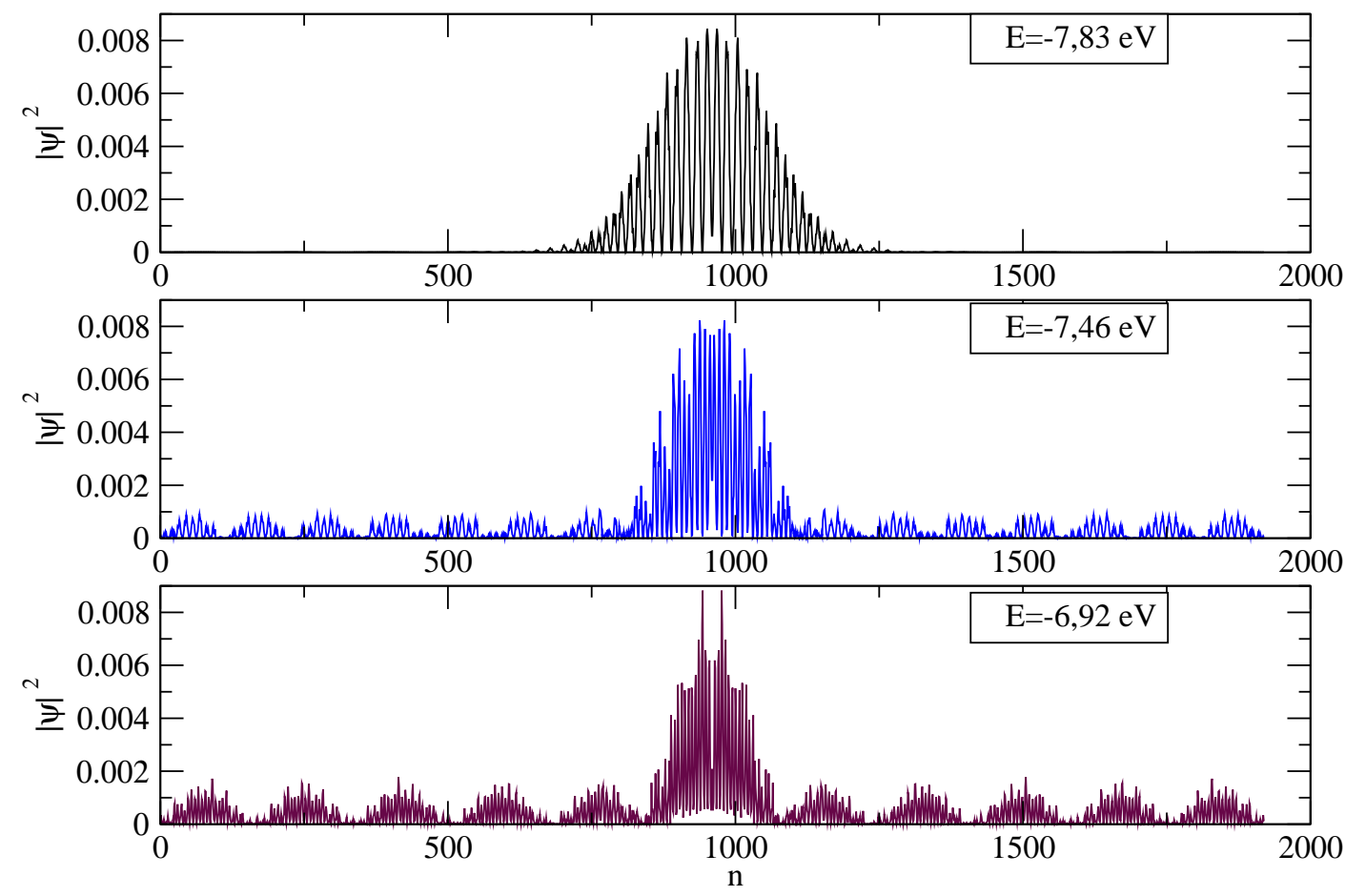

Figura 3.10: Probabilidad de ocupación de tres estados cuasi-localizados para energías $E=-7,83 \mathrm{eV}, E=-7,46 \mathrm{eV}$ y $E=-6,92 \mathrm{eV}$, que se corresponden con tres de las autofunciones en la figura 3.9. El índice $n$ etiqueta los átomos de la estructura $(6,6) / 4(9,9) /(6,6)$ de izquierda a derecha. Como vemos, estas funciones de onda se localizan mayoritariamente en el interior de la cavidad. Existen otras ondas localizadas en los extremos del nanotubo finito empleado para calcular las autofunciones y autoenergías. 


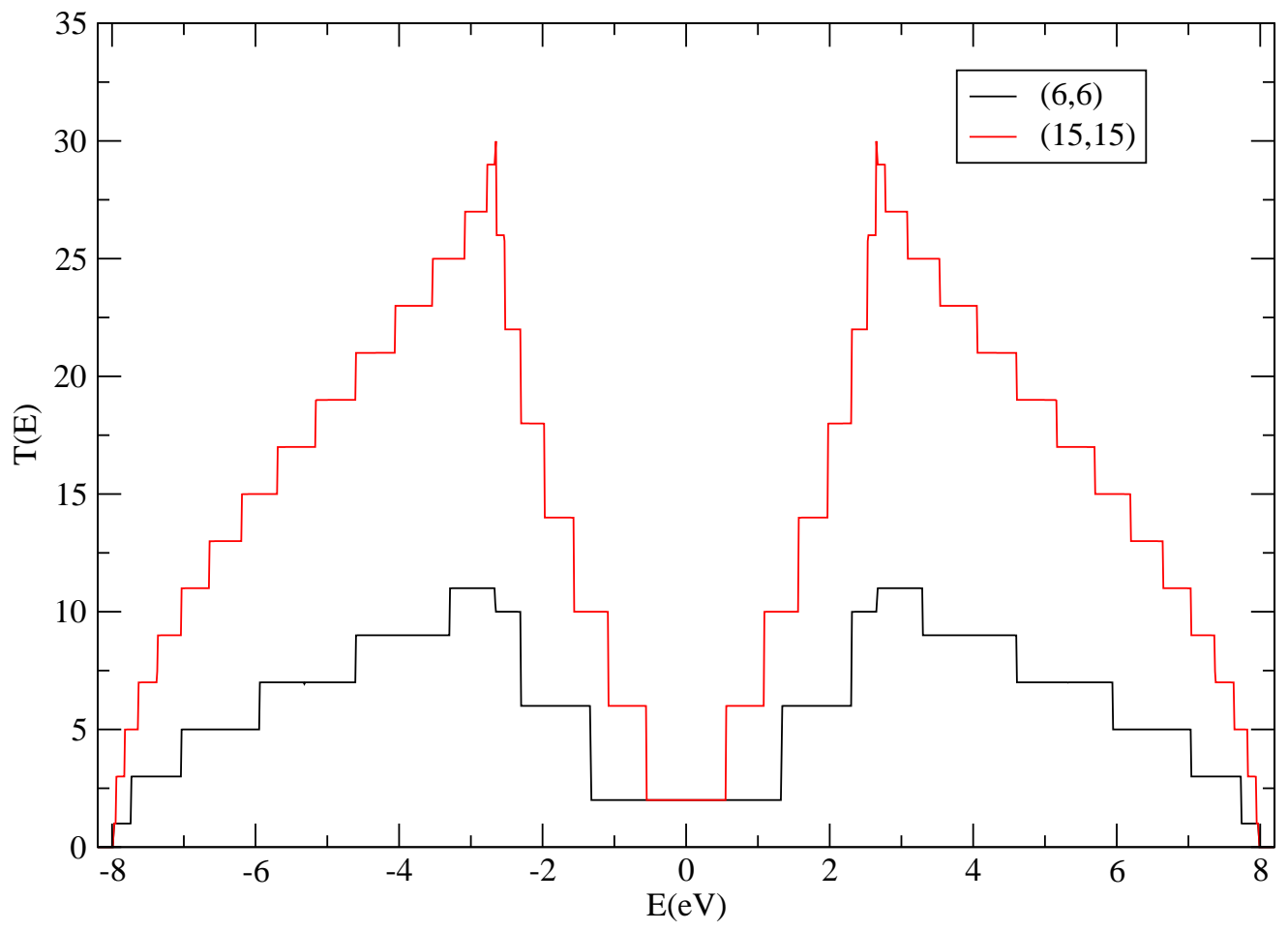

Figura 3.11: Funciones de transmisión en función de la energía de los nanotubos puros $(6,6)$, en color negro, y $(15,15)$, en color rojo. El nanotubo $(15,15)$ presenta en todo el espectro un número de canales mucho mayor que el del $(6,6)$, excepto en una pequeña región alrededor de $E=0$ y en los extemos de la energía. 


\subsection{Influencia del diámetro de la cavidad en la fun- ción de transmisión}

En el apartado 3.2 hemos estudiado las propiedades asociadas a la estructura $(6,6) / 4(9,9) /(6,6)$. La aparición de anti-resonancias en la función de transmisión nos ha llevado a la conclusión de que en el interior de la cavidad se producen estados cuasi-localizados directamente asociados a las diferencias entre la estructura de bandas de los contactos y de la cavidad. En concreto, a que el número de canales en los contactos es menor que en la cavidad. Ha de existir por tanto una fuerte dependencia de la transmisión con el diámetro de la cavidad, ya que el número de canales en la misma aumenta con el diámetro.

En este apartado vamos a obtener la función de transmisión en una cavidad $(6,6) / 4(15,15) /(6,6)$. Compararemos los resultados con los obtenidos para la estructura $(6,6) / 4(9,9) /(6,6)$, estudiando la dependencia de la transmisión con el diámetro de la cavidad. Mostramos en primer lugar, en la figura 3.11, la función de transmisión de los nanotubos puros $(6,6)$ y $(15,15)$. La diferencia entre el número de canales de los nanotubos puros $(6,6)$ y $(15,15)$ es mucho más significativa que entre los nanotubos $(6,6)$ y $(9,9)$. Esto debe dar lugar a que se produzcan un mayor número de resonancias y anti-resonancias en la transmisión de la cavidad.

En la figura 3.12 mostramos la función de transmisión en función de la energía para la estructura $(6,6) / 4(15,15) /(6,6)$. En la parte superior se representa $T(E)$ en el intervalo de energías $-6 \mathrm{eV}<E<6 \mathrm{eV}$. Es claro que aparece un gran número de dips en la transmisión, en comparación a la estructura $(6,6) / 4(9,9) /(6,6)$. Esto es debido, como ya hemos mencionado, a la enorme diferencia de canales entre el nanotubo que conforma la cavidad y los de los contactos. Existe, por tanto, un gran número de canales localizados en la cavidad que dan lugar a interferencias destructivas entre dichos canales y los extendidos a lo largo de toda la estructura, lo que genera las anti-resonancias que se observan en la figura. 

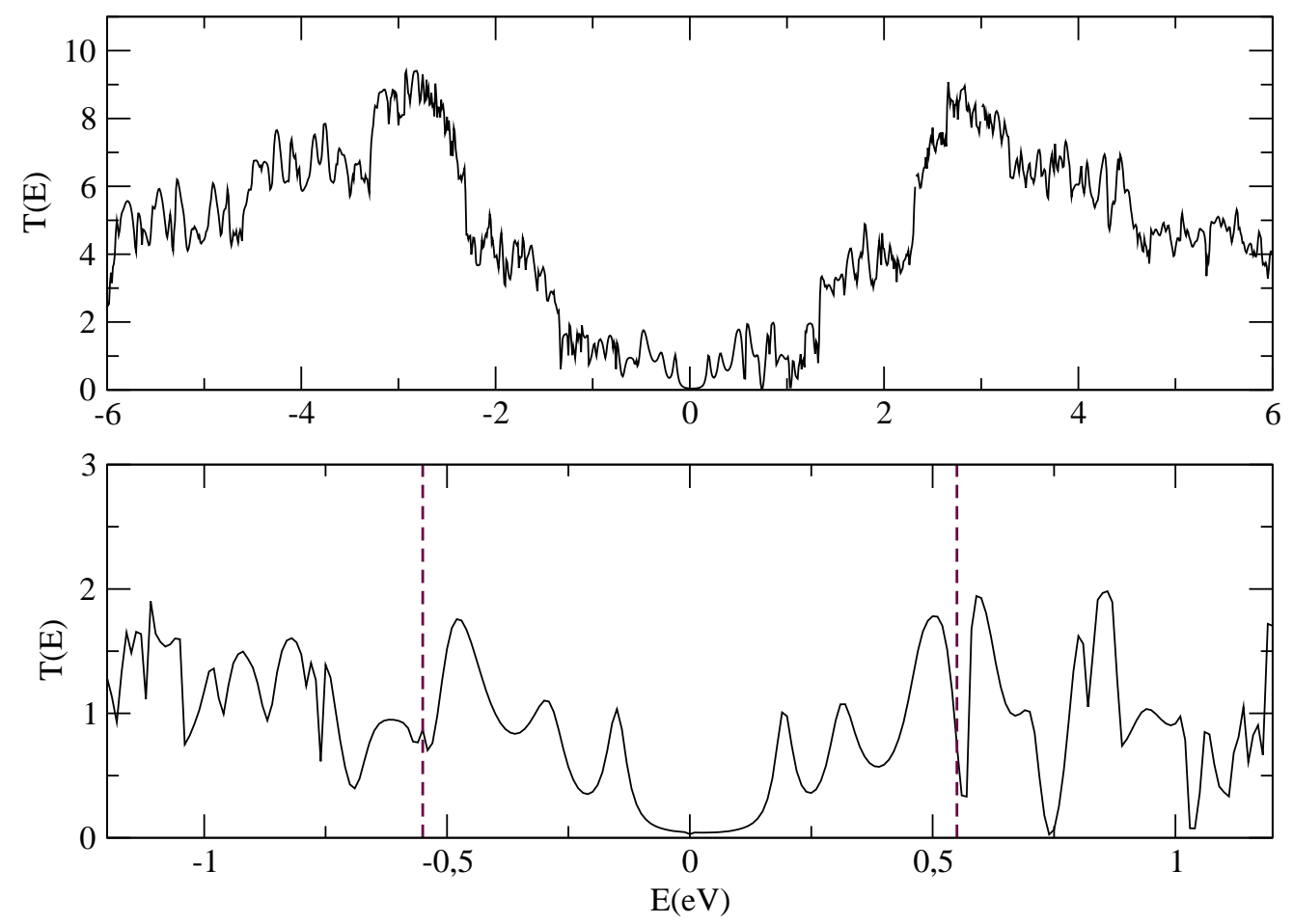

Figura 3.12: Función de transmisión de la cavidad $(6,6) / 4(15,15) /(6,6)$ en función de la energía. En la parte superior de la figura se muestra la región comprendida en el rango $-6 \mathrm{eV}<E<6 \mathrm{eV}$. La enorme diferencia de canales entre los nanotubos $(6,6)$ y $(15,15)$ hace que exista un gran número de dips en la transmisión. La parte inferior muestra una ampliación a la región central de la función de transmisión. Las líneas discontinuas verticales delimitan la región de energías en la cual el número de canales para los dos nanotubos puros que componen la estructura es el mismo. 
En la parte inferior de la figura 3.12 mostramos una ampliación de la figura superior al entorno de la región donde coincide el número de canales de los dos nanotubos que conforman la estructura, el rango de energías $-0,55 \mathrm{eV}<E<0,55 \mathrm{eV}$. Se producen en esta región resonancias muy pronunciadas como consecuencia de la superposición entre las ondas reflejadas en las uniones de ambos nanotubos.

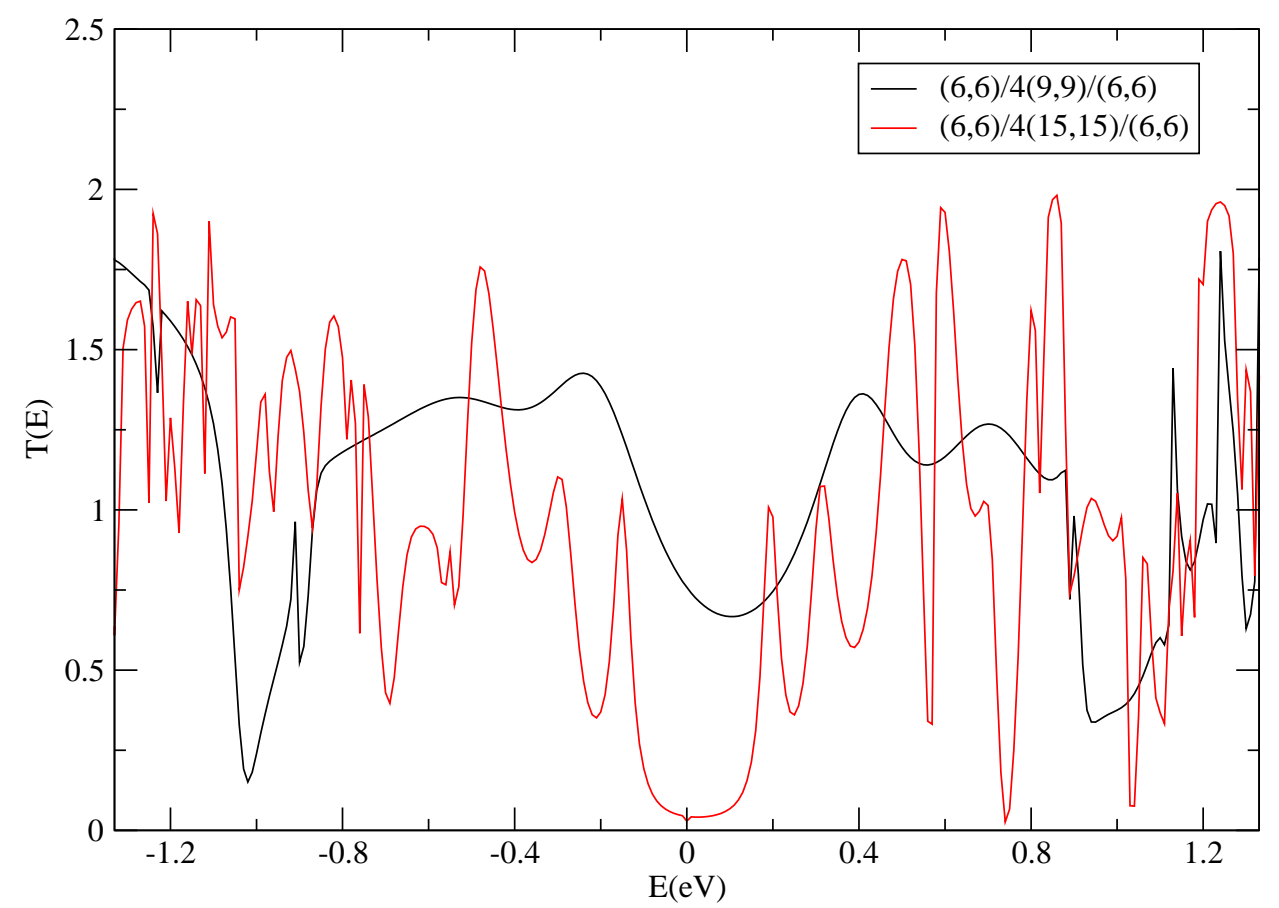

Figura 3.13: Funciones de transmisión en función de la energía de las cavidades $(6,6) / 4(9,9) /(6,6)$ (color negro) y $(6,6) / 4(15,15) /(6,6)$ (color rojo). Las resonancias de esta última función son más pronunciadas.

En la figura 3.12 podemos ver, además, otro hecho interesante en torno a la región de Fermi que merece la pena estudiar. Si nos fijamos en dicha región, vemos que se produce una caida del valor de la transmisión hasta tomar prácticamente un valor cero. Puesto que los dos nanotubos que conforman la estructura son con- 
ductores, la explicación a este hecho no se debe a este carácter conductor de los nanotubos, sino a la enorme diferencia en anchura entre ambos nanotubos, que hace que las regiones de transición que producen la unión entre ellos sean muy grandes, existiendo reflexiones también muy grandes en dichas regiones de transición. Tenemos transmisión en las energías para las que existen estados resonantes dentro de la cavidad. Esta circunstancia no se producía de forma tan significativa en el caso de la estructura $(6,6) / 4(9,9) /(6,6)$, como podemos comparar en la gráfica 3.13, donde se muestran las funciones de transmisión de ambas estructuras. Vemos además en la figura 3.13 que las resonancias y anti-resonancias que muestra la estructura $(6,6) / 4(15,15) /(6,6)$ son mucho más pronunciadas que las asociadas a la cavidad $(6,6) / 4(9,9) /(6,6)$.

\subsection{Cavidades de semiconductores}

En los apartados anteriores se ha realizado un estudio de las propiedades de transmisión en cavidades formadas por CNT tipo armchair, de carácter metálico. Los resultados obtenidos, como la aparición de resonancias y anti-resonancias en la función de transmisión, no dependen explícitamente de la quiralidad de los nanotubos que forman la estructura, así como tampoco de su carácter conductor. De esta manera, los resultados han de ser reproducibles en el caso de que dicha estructura esté compuesta por nanotubos semiconductores de geometría quiral. En el presente apartado obtendremos la función de transmisión y la densidad de estados para cavidades formadas por un semiconductor con geometría quiral, en concreto el $(5,4)$. En particular, mostraremos los resultados obtenidos para la estructura $(5,4) / 4(6,6) /(5,4)$, una cavidad metálica rodeada por dos nanotubos semiconductores, y para la estructura $(5,2) / 5(6,6) /(5,4)$, una cavidad metálica con un nanotubo semiconductor a un lado y uno metálico al otro lado. 
3.4.1. Cavidad $(5,4) / 4(6,6) /(5,4)$

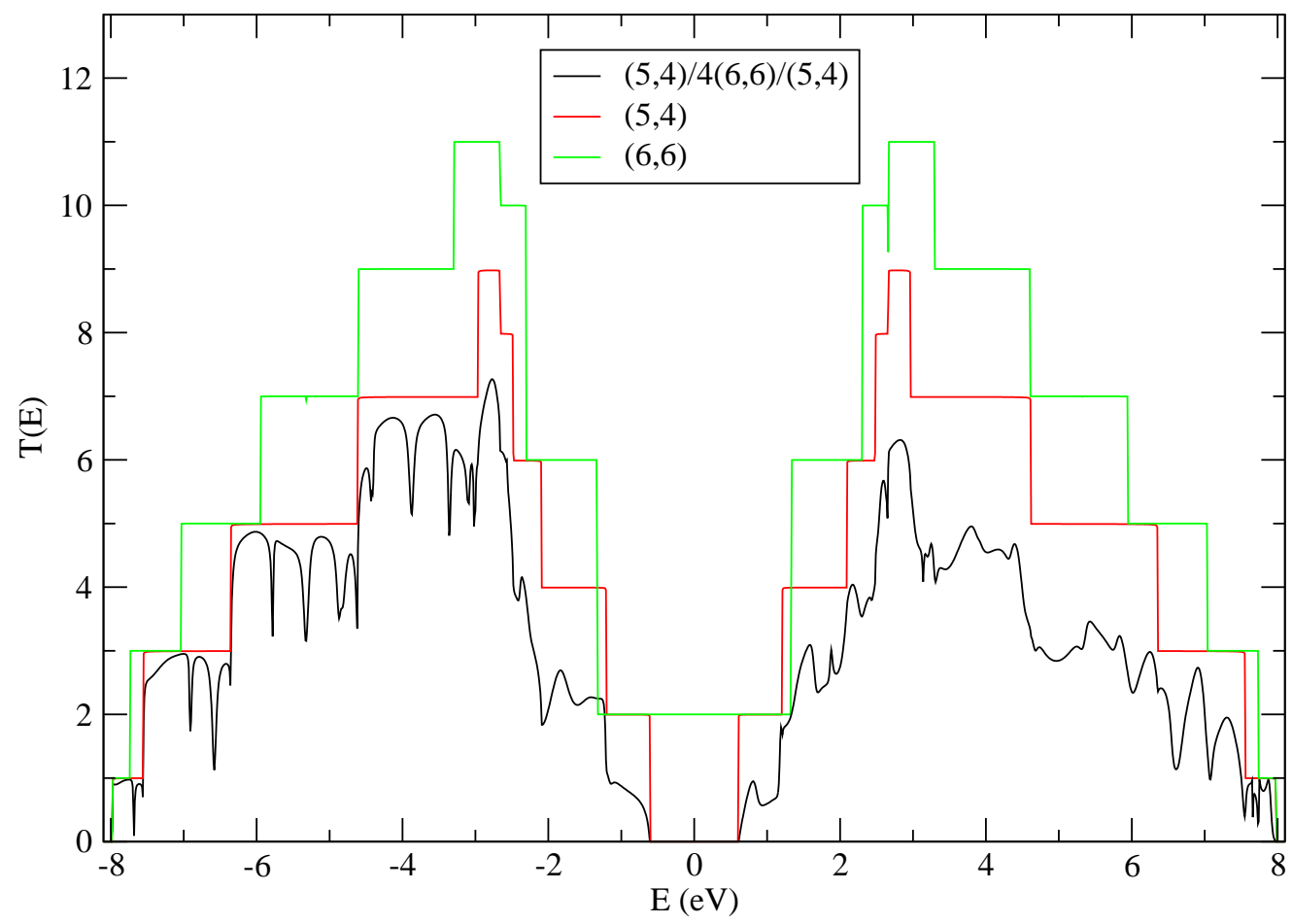

Figura 3.14: Función de transmisión para los nanotubos $(6,6)$ y $(5,4)$, junto con la estructura $(5,4) / 4(6,6) /(5,4)$. El número de canales del nanotubo central es mayor o igual que el de los nanotubos de los extremos en casi todo el rango de energías, por lo que se producen anti-resonancias en la función de trasmisión.

Realizaremos en este apartado el estudio de la cavidad formada por dos contactos semiconductores $(5,4)$ junto con la cavidad $(6,6)$. Esta nueva estructura es completamente diferente a las anteriores. En primer lugar, porque utilizamos nanotubos con geometría quiral como contactos en lugar de armchair. En segundo lugar, porque los nanotubos utilizados, $(5,4)$, son semiconductores, luego las propiedades 
de conducción son completamente diferentes a los casos estudiados anteriormente.

En la figura 3.14 se muestra la función de transmisión para la estructura con una cavidad $(5,4) / 4(6,6) /(5,4)$, junto con la función de transmisión para los CNT $(6,6)$ y $(5,4)$ infinitos. Como vemos en dicha figura, el número de canales de transmisión para el $(5,4)$ es menor o igual que el del $(6,6)$ en casi todo el rango de energías, por lo que se producen también aquí anti-resonancias en la función de transmisión de la cavidad $(5,4) / 4(6,6) /(5,4)$. Sin embargo, el hecho de que los nanotubos utilizados como contactos sean semiconductores impide en este caso el estudio de las oscilaciones que dan lugar a resonancias en la transmisión alrededor del nivel de Fermi, ya que existe un gap en torno al mismo.

En la figura 3.15 se muestra la función de transmisión junto con la densidad local de estados en la cavidad para la estructura $(5,4) / 4(6,6) /(5,4)$. Puede comprobarse en dicha figura que las anti-resonancias en la función de transmisión coinciden con los picos en la densidad de estados en el interior de la cavidad. Así pues, dichas antiresonancias se relacionan directamente con la aparición de estados en el interior de la cavidad, como en el caso anterior. En la figura 3.16 se comprueba, además, que no aparecen dichos estados en la densidad de estados en los contactos de la estructura. En la LDOS de los contactos sólo se aprecian las singularidades de Van-Hove.

Llama la atención en ambas figuras la existencia de estados en la región de energías correspondiente al gap en la transmisión. En particular, para el valor $E=0,24$ $\mathrm{eV}$ aparece un pico que indica la existencia de estados completamente confinados en la cavidad, como podemos comprobar en la figura 3.16, donde vemos la LDOS de la cavidad y de los contactos. En estas figuras se observa claramente que los estados cuasi-localizados se encuentran en la cavidad ya que no aparecen en la LDOS de los contactos. Se trata, por tanto, de un punto cuántico, similar al que se describe en el trabajo de L. Chico et al. (1998). 


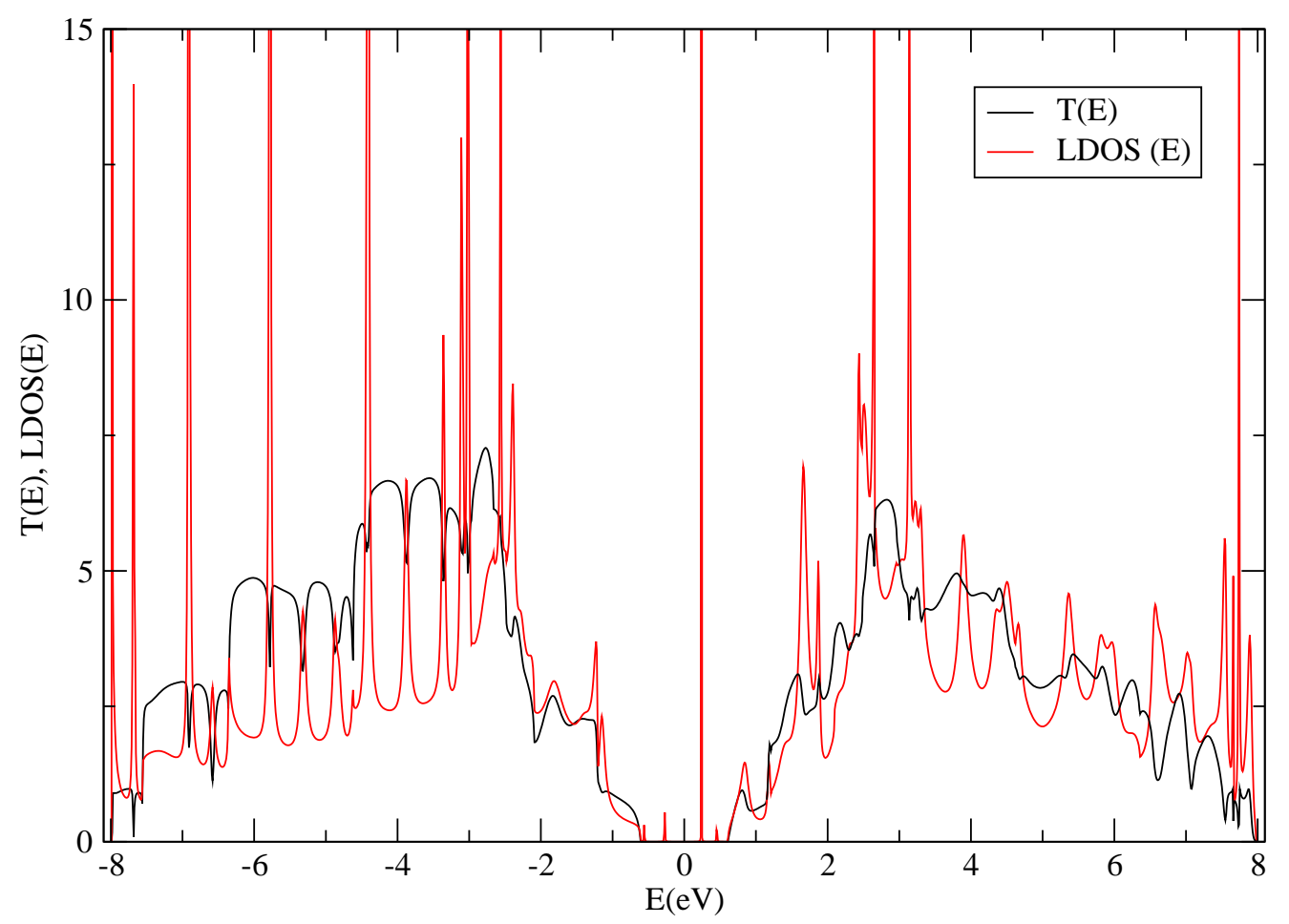

Figura 3.15: Función de transmisión y densidad local de estados en el interior de la cavidad para la estructura $(5,4) / 4(6,6) /(5,4)$. Los dips en la transmisión se corresponden con los máximos de la densidad local de estados. Se aprecia claramente un pico en la densidad local de estados alrededor de $E=0,24 \mathrm{eV}$, dentro del gap. Esto indica que el sistema se podría comportar como un punto cuántico. 
El comportamiento de nanotubos de carbono como puntos cuánticos ha despertado grandes expectativas en la comunidad científica, ya que son numerosas las posibles aplicaciones prácticas a las que pueden dar lugar. Algunas de dichas aplicaciones pueden resultar de gran relevancia, como ya mencionamos anteriormente en el apartado 1.7 .

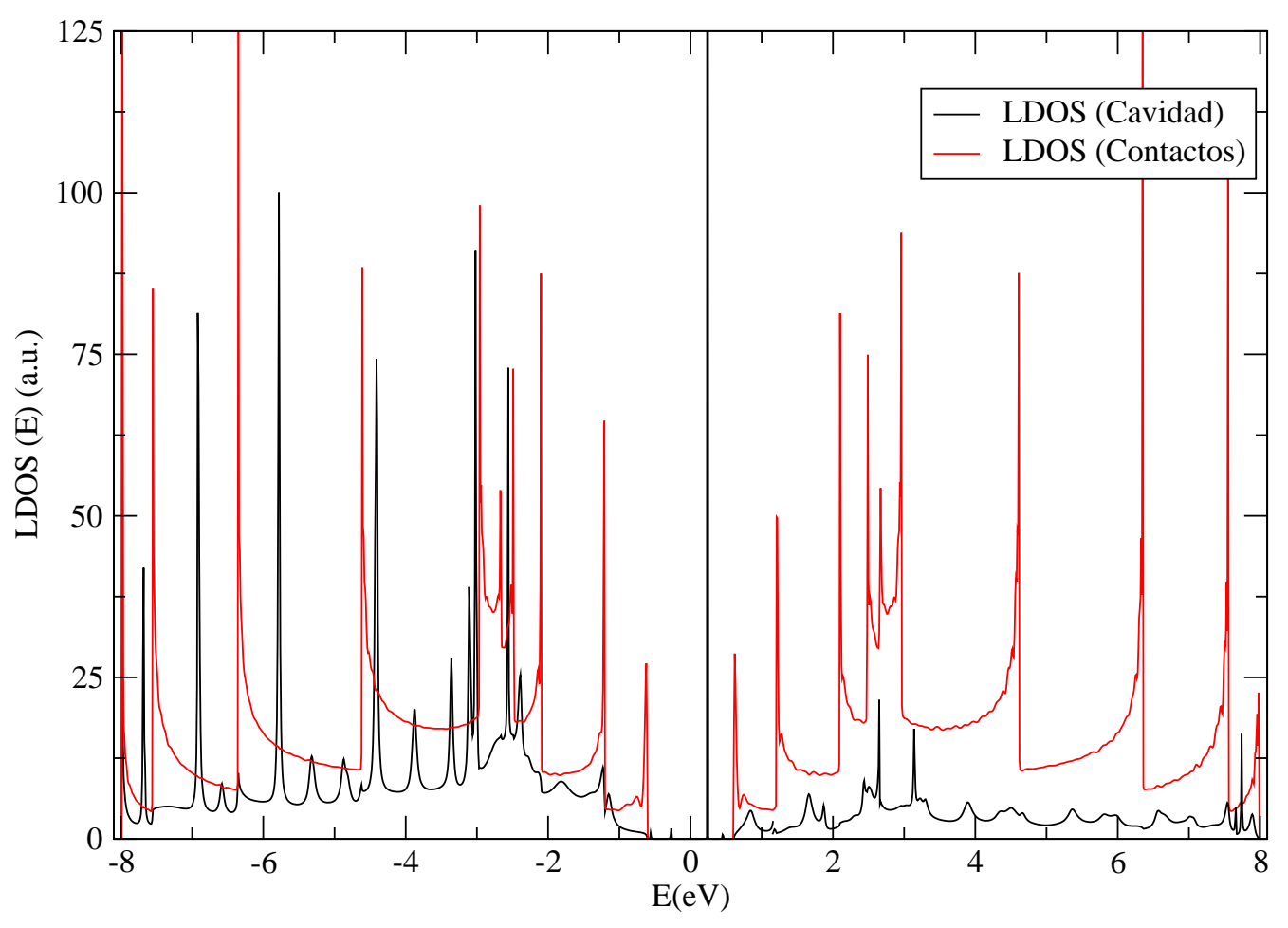

Figura 3.16: Densidad local de estados en el interior de la cavidad $(5,4) / 4(6,6) /(5,4)$ y en los contactos. La figura muestra claramente la existencia de estados localizados en el interior de la cavidad. 


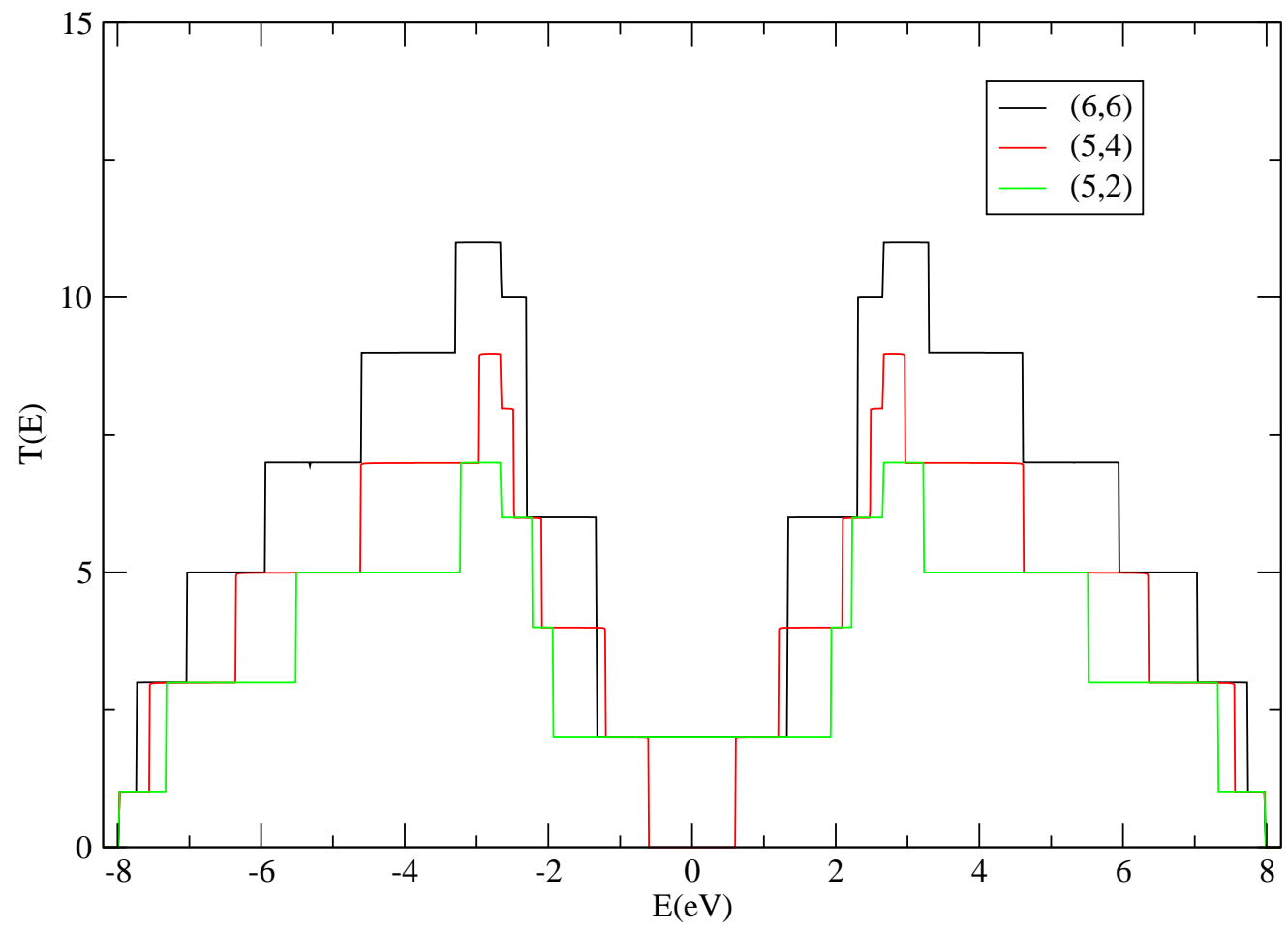

Figura 3.17: Función de transmisión para los tres nanotubos que componen la estructura $(5,2) / 5(6,6) /(5,4)$. El número de canales del nanotubo central es casi en la totalidad del rango de energías mayor o igual que el de los nanotubos de los extremos. 


\subsubsection{Cavidad $(5,2) / 5(6,6) /(5,4)$}

En este apartado estudiaremos la cavidad $(5,2) / 5(6,6) /(5,4)$. Se trata de una estructura asimétrica que se compone de tres tipos de nanotubos. El interior de la cavidad está nuevamente formado por el CNT $(6,6)$, que se comporta como un conductor. Los contactos están formados por un nanotubo conductor y otro semiconductor, el $(5,2)$ y el $(5,4)$, respectivamente (ambos con geometría quiral).

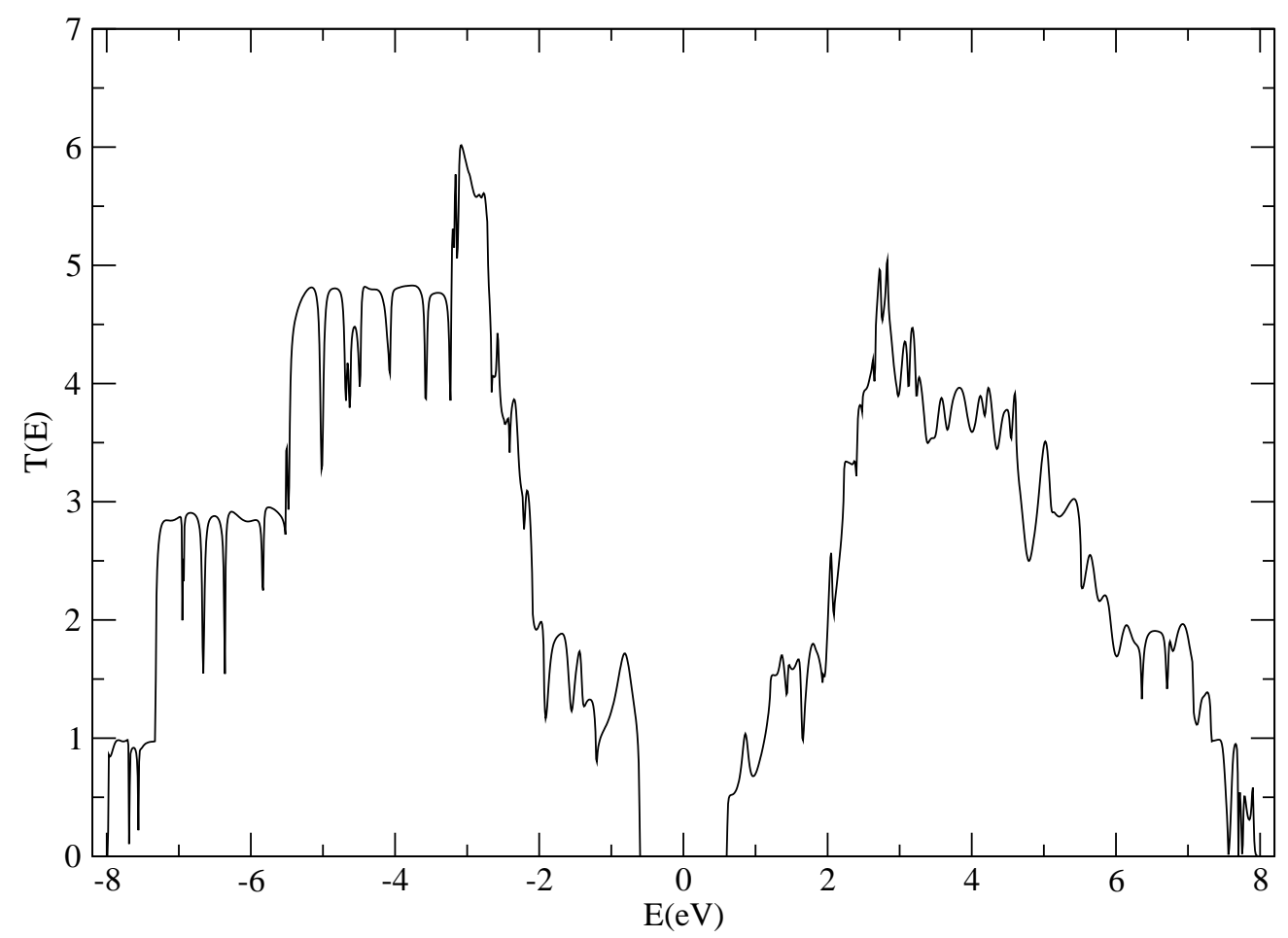

Figura 3.18: Función de transmisión de la estructura $(5,2) / 5(6,6) /(5,4)$, donde aparecen anti-resonancias de la misma naturaleza que en la cavidad $(6,6) / 4(9,9) /(6,6)$.

Presentamos en primer lugar en la figura 3.17 la función de transmisión de los 
tres nanotubos utilizados en nuestro cálculo. Comprobamos de este modo que la nueva estructura posee las mismas características que las anteriormente estudiadas en cuanto al número de canales. Como vemos en dicha figura, en ambos contactos el número de canales es casi en la totalidad del rango de energías menor o igual que el número de canales en el nanotubo central, por lo que se han de producir también aquí anti-resonancias en la función de transmisión.

La función de transmisión de la estructura $(5,2) / 5(6,6) /(5,4)$ se muestra en la figura 3.18. En dicha figura comprobamos la aparición de anti-resonancias, como esperábamos, y que esta estructura muestra similares características a la de la cavidad $(5,4) / 4(6,6) /(5,4)$ estudiada anteriormente, con la aparición de anti-resonancias que han de tener idéntico origen que en los casos ya estudiados. Nuevamente, el hecho de que uno de los nanotubos utilizados sea semiconductor hace que aparezca un gap de energía en torno a la región de Fermi, que impide el estudio de las oscilaciones que dan lugar a resonancias en la transmisión alrededor de dichas energías.

Para completar nuestro estudio calcularemos la densidad local de estados en un extremo de la estructura y en el interior de la cavidad, que se muestran en la figura 3.19, junto con la función de transmisión de toda la estructura. Como vemos en dicha figura, los dips en la transmisión se corresponden claramente con los picos de la densidad local de estados en la cavidad. Además, vemos que estos picos no aparecen en la LDOS del contacto $(5,2)$, donde sólo apreciamos las singularidades de Van-Hove, como era de esperar debido a que sabemos que los estados localizados se encuentran en la cavidad. No se muestra LDOS en el contacto $(5,4)$, similar a la del $(5,2)$, a fin de que la figura sea lo más clara posible.

Puede apreciarse también en la figura 3.19, un pequeño pico de la densidad local de estados en el interior de la cavidad, que se asemeja a lo que podría ser un punto cuántico. En este caso los efectos están suavizados y la densidad local de estados no llega a ser cero a ambos lados de dicho pico, por lo que no podemos hablar de un es- 


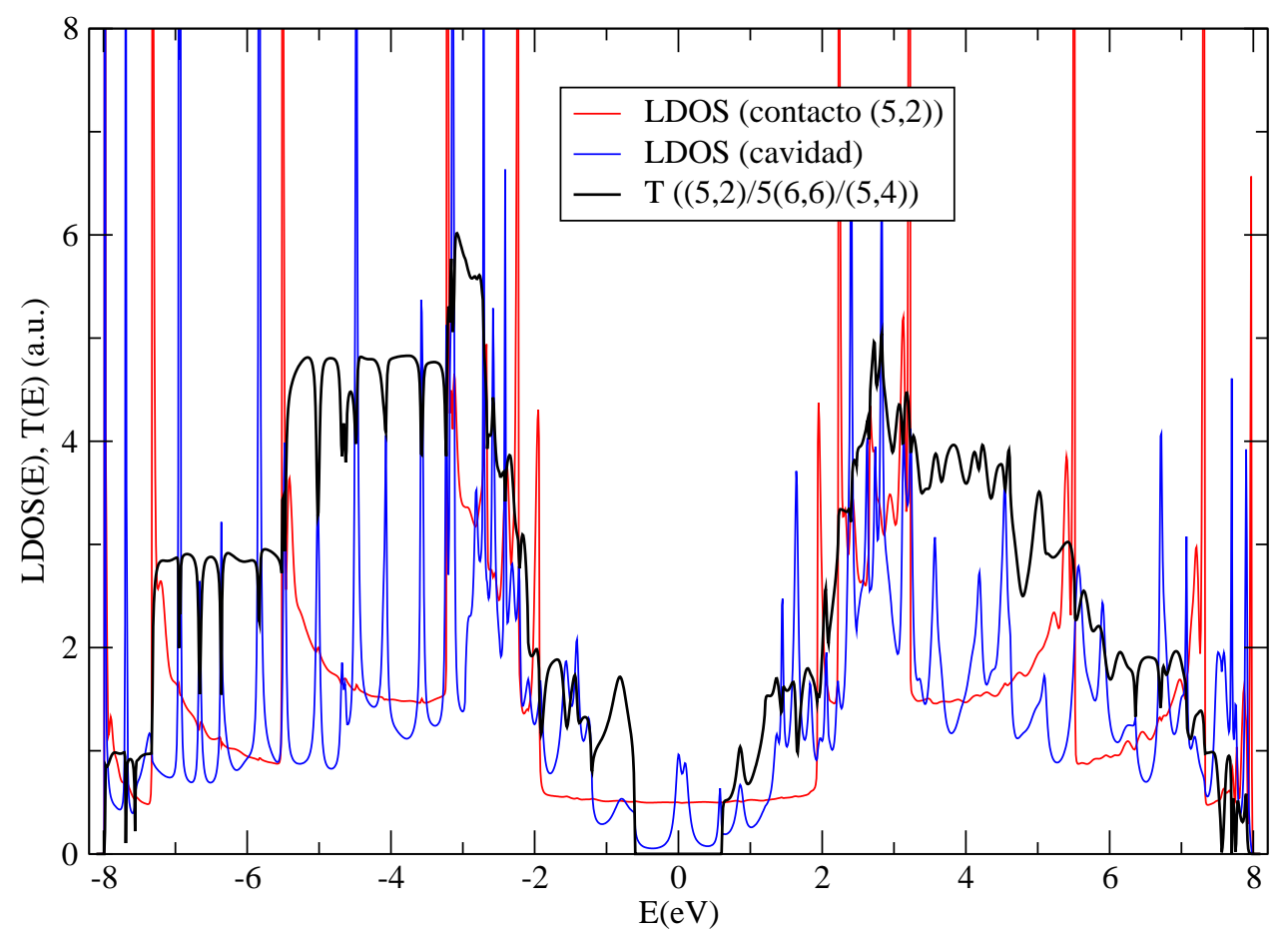

Figura 3.19: Función de transmisión de la estructura $(5,2) / 5(6,6) /(5,4)$ (curva en color negro), junto con la densidad local de estados en dos regiones de la estructura. La curva de color azul se corresponde con LDOS en la cavidad. La curva en color rojo muestra LDOS en el contacto $(5,2)$. 
tado completamente confinado, como en el caso de la estructura $(5,4) / 4(6,6) /(5,4)$. Esto se debe a que en este caso uno de los contactos es metálico. Vemos aquí también que la presencia de anti-resonancias en cavidades semiconductoras se deben a la existencia de estados cuasi-localizados en en interior de la cavidad, de la misma forma que observamos para la cavidad $(6,6) / 4(9,9) /(6,6)$.

Las uniones $(5,2) /(6,6)$ y $(6,6) /(5,4)$ no son rectas sino que los nanotubos forman un ángulo mayor que cero entre sí. Esto hace que no tengamos simetría alrededor del eje del nanotubo $(6,6)$. Tanto la estructura $(5,4) / 5(6,6) /(5,4)$ anteriormente estudiada como la $(5,2) / 5(6,6) /(5,4)$ podemos hacerlas de diversos modos rotando una de las uniones alrededor del eje del nanotubo $(6,6)$. En particular, es posible girar un ángulo de $60^{\circ}$ respecto a la posición inicial, obteniendo de este modo una geometría diferente. Por lo tanto, existen en total 4 maneras diferentes de acoplamiento entre las dos uniones. No debemos obviar el hecho de que en este tipo de estructuras los resultados podrían presentar cierta dependencia con el ángulo formado en la misma. Veamos a continuación esta dependencia para la estructura $(5,2) / 5(6,6) /(5,4)$. Si denotamos a la geometría ya estudiada como $(5,2) / 5(6,6) /(5,4)_{0^{\circ}}$, tenemos además las posibilidades $(5,2) / 5(6,6) /(5,4)_{60^{\circ}}$, $(5,2) / 5(6,6) /(5,4)_{120^{\circ}} \mathrm{y}(5,2) / 5(6,6) /(5,4)_{180^{\circ}}$.

En la figura 3.20 se representa como ejemplo, una parte de la función de transmisión para las cuatro geometrías mencionadas con diferentes colores. Como puede apreciarse en la misma, aunque el comportamiento global de la función de transmisión es prácticamente idéntico para las cuatro geometrías, existen ligeras diferencias como consecuencia de la diferente orientación relativa de las cuatro estructuras. Sin embargo, estas diferencias prácticamente no afectan a los estados cuasi-localizados que se forman en el interior de la cavidad. La figura 3.21 muestra un comportamiento similar para la densidad local de estados en el interior de la cavidad. En esta figura mostramos una parte de la LDOS, donde se aprecia un ligero desplazamiento en energías para los picos que presenta dicha función. 


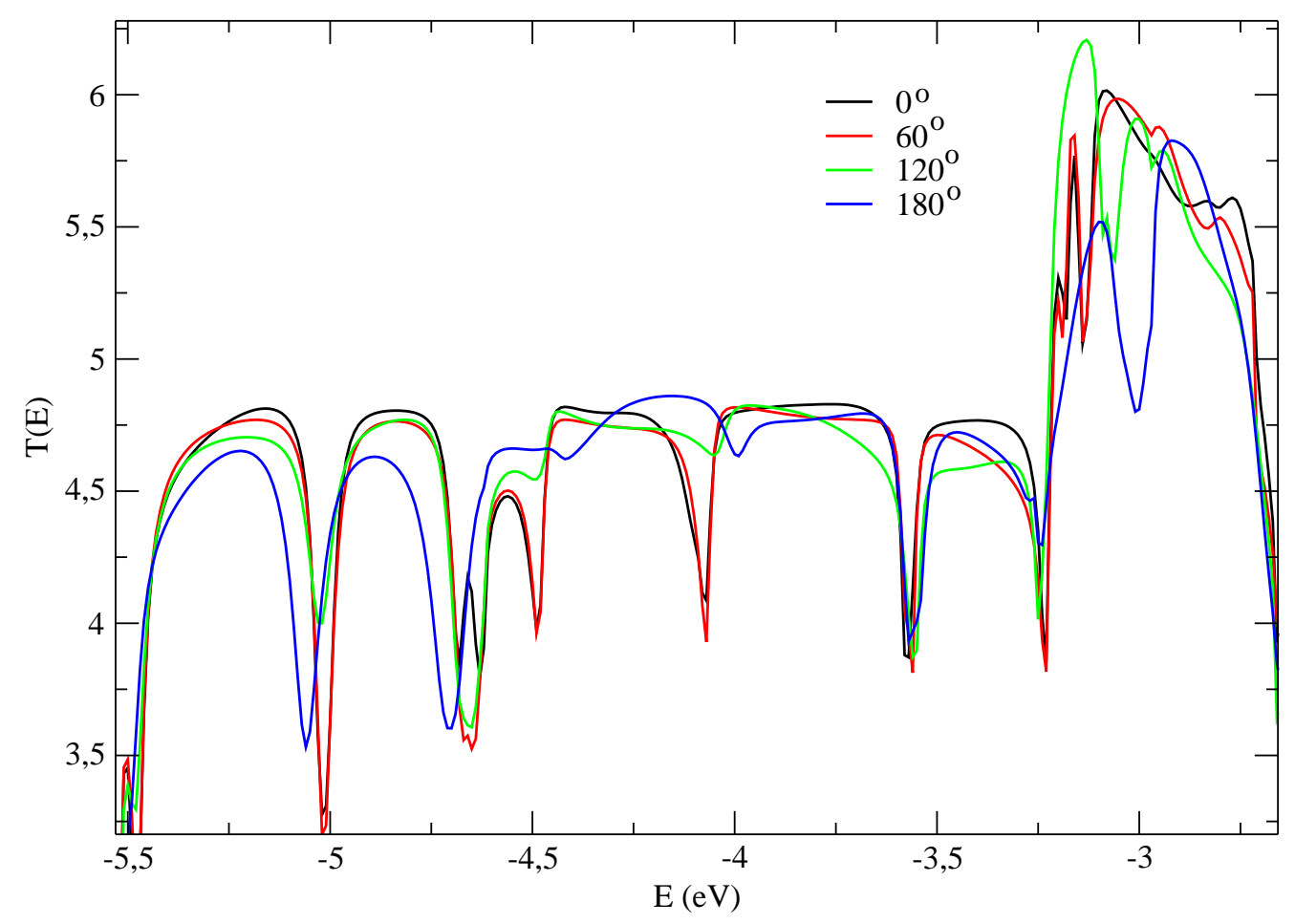

Figura 3.20: Función de transmisión de la estructura $(5,2) / 5(6,6) /(5,4)$ para diferentes ángulos de acoplamiento. Se muestra sólo una parte de la misma por claridad, el rango de energías $-5,5 \mathrm{eV}<E<-2,7 \mathrm{eV}$. El comportamiento es similar en el resto del rango de energías. Se aprecian únicamente ligeras diferencias entre las cuatro curvas, que se deben a la diferente geometría de las cuatro estructuras. Estas pequeñas diferencias no afectan al comportamiento global de la función de transmisión. 


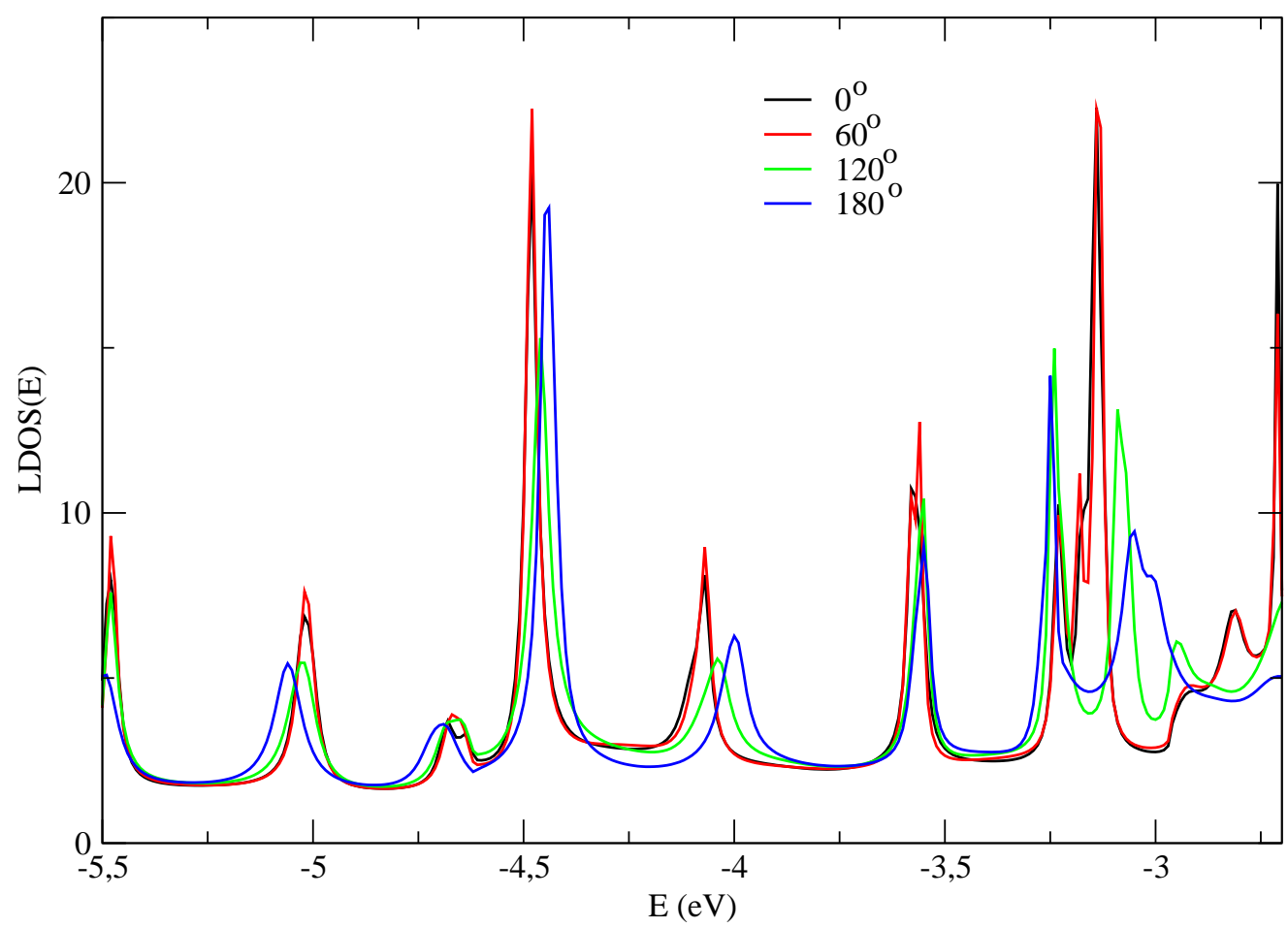

Figura 3.21: Densidad local de estados en el interior de la cavidad de la estructura $(5,2) / 5(6,6) /(5,4)$ para diferentes ángulos de acoplamiento. Se muestra, a efectos de que la figura sea lo más clara posible, el rango de energías $-5,5 \mathrm{eV}<E<-2,7 \mathrm{eV}$, aunque el comportamiento es similar en todo el rango. Se aprecia un ligero desplazamiento en energías para los picos de la LDOS. 


\subsection{Estudio de un estrechamiento: nanoestructura $(9,9) / 4(6,6) /(9,9)$}
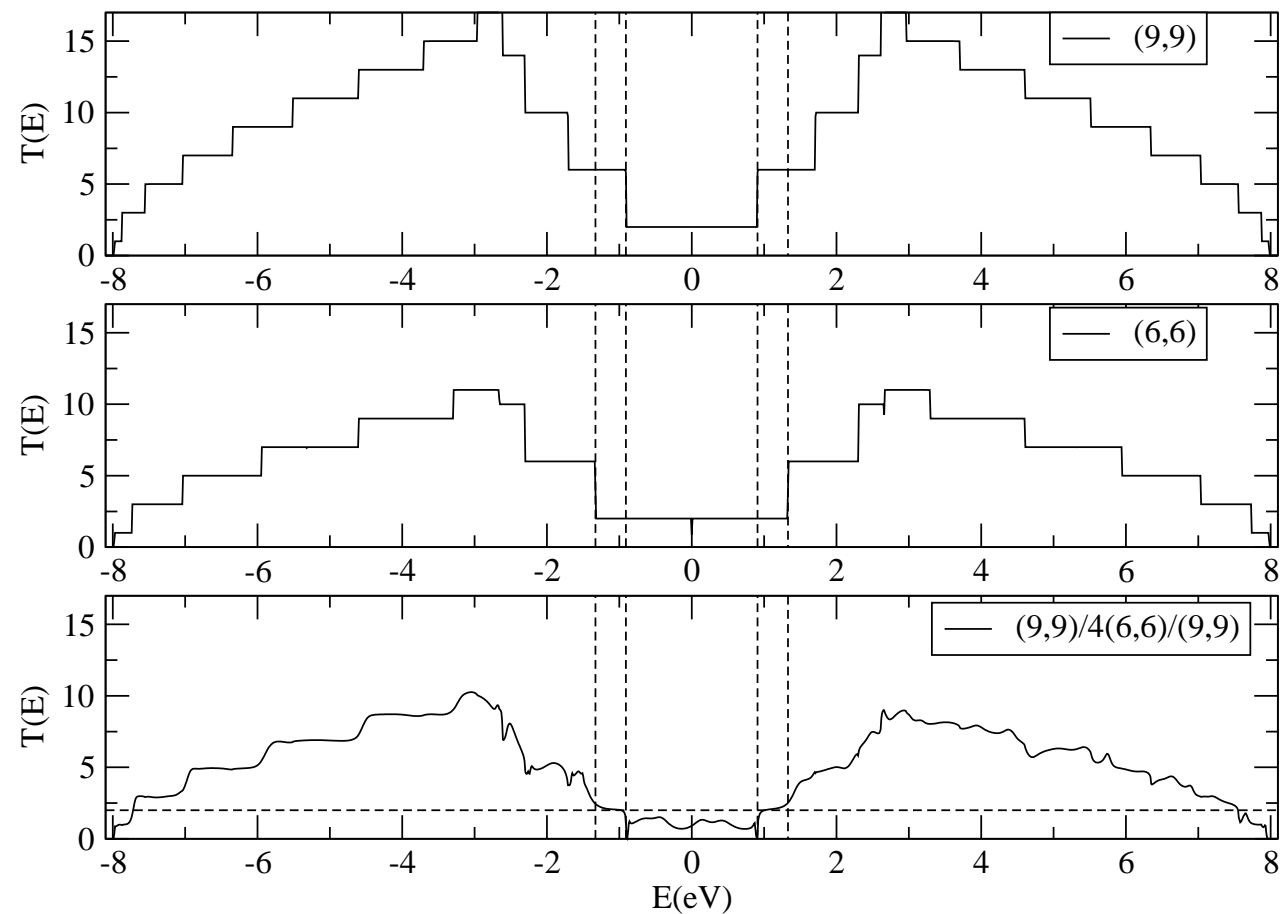

Figura 3.22: Funciones de transmisión del nanotubo infinito $(9,9)$, parte superior, del nanotubo infinito $(6,6)$, parte central, y de la estructura $(9,9) / 4(6,6) /(9,9)$, parte inferior. Las líneas verticales discontinuas delimitan la región donde el número de canales del nanotubo $(9,9)$ es igual a cuatro mientras que el del estrechamiento, nanotubo $(6,6)$, es igual a dos (rango de energías $0,91 \mathrm{eV}<|E|<1,33 \mathrm{eV})$.

Vamos a estudiar en este apartado un sistema del tipo $(n, n) / N(m, m) /(n, n)$, pero el caso opuesto al estudiado en el apartado 3.2. Es decir, trabajaremos con un sistema donde el número de canales de los contactos es mayor o igual al de la parte 
central en todo el rango de energías. En este caso el diámetro de la parte central es menor que el de los extremos, por lo que nos referiremos a dicha región como estrechamiento en lugar de cavidad. Para formar nuestra estructura, tomaremos nuevamente los nanotubos $(9,9)$ y $(6,6)$, pero utilizando ahora el $(6,6)$ como parte central en la estructura y el $(9,9)$ como contactos en los extremos de la misma. Tenemos así la estructura $(9,9) / 4(6,6) /(9,9)$.

En la figura 3.22 mostramos de nuevo las funciones de transmisión del nanotubo infinito $(9,9)$, del $(6,6)$ y también de la estructura $(9,9) / 4(6,6) /(9,9)$, en las regiones superior, central e inferior, respectivamente, de dicha figura. No se aprecian en este caso dips en la transmisión que pudieran indicar la existencia de estados cuasi-localizados. Esto es debido a que en este caso el número de canales en el estrechamiento central de la estructura es siempre menor o igual que en los contactos, lo que no favorece la aparición de estados localizados en la parte central del nanotubo.

En la región en torno a la energía de Fermi el comportamiento es similar al caso $(6,6) / 4(9,9) /(6,6)$. Se aprecian oscilaciones en la función de transmisión debidas a las múltiples reflexiones en las dos uniones de los nanotubos. Sin embargo, en las regiones delimitadas por líneas verticales discontinuas $(0,91 \mathrm{eV}<|E|<1,33 \mathrm{eV})$ la situación es completamente diferente. Estas dos regiones se corresponden con los valores de la energía para los que el número de canales del nanotubo $(9,9)$ (contactos) es igual a cuatro mientras que el del nanotubo $(6,6)$ (estrechamiento) es igual a dos. Dentro de este rango de energías la función de transmisión llega a ser superior a dos, lo que no sucedía en el caso del $(6,6) / 4(9,9) /(6,6)$. Esto indica que existen ondas evanescentes que se transmiten por efecto túnel a través del estrechamiento, además de los dos canales propios del mismo. Podemos comprobar esta afirmación estudiando la transmisión en la misma estructura pero alargando la parte central (correspondiente al nanotubo $(6,6)$ ), lo que disminuye la probabilidad de transmisión por efecto túnel. Sí que se aprecian ciertos picos en esa región debido a la existencia de estados resonantes en la parte central lo que facilita el efecto túnel por resonancia. 


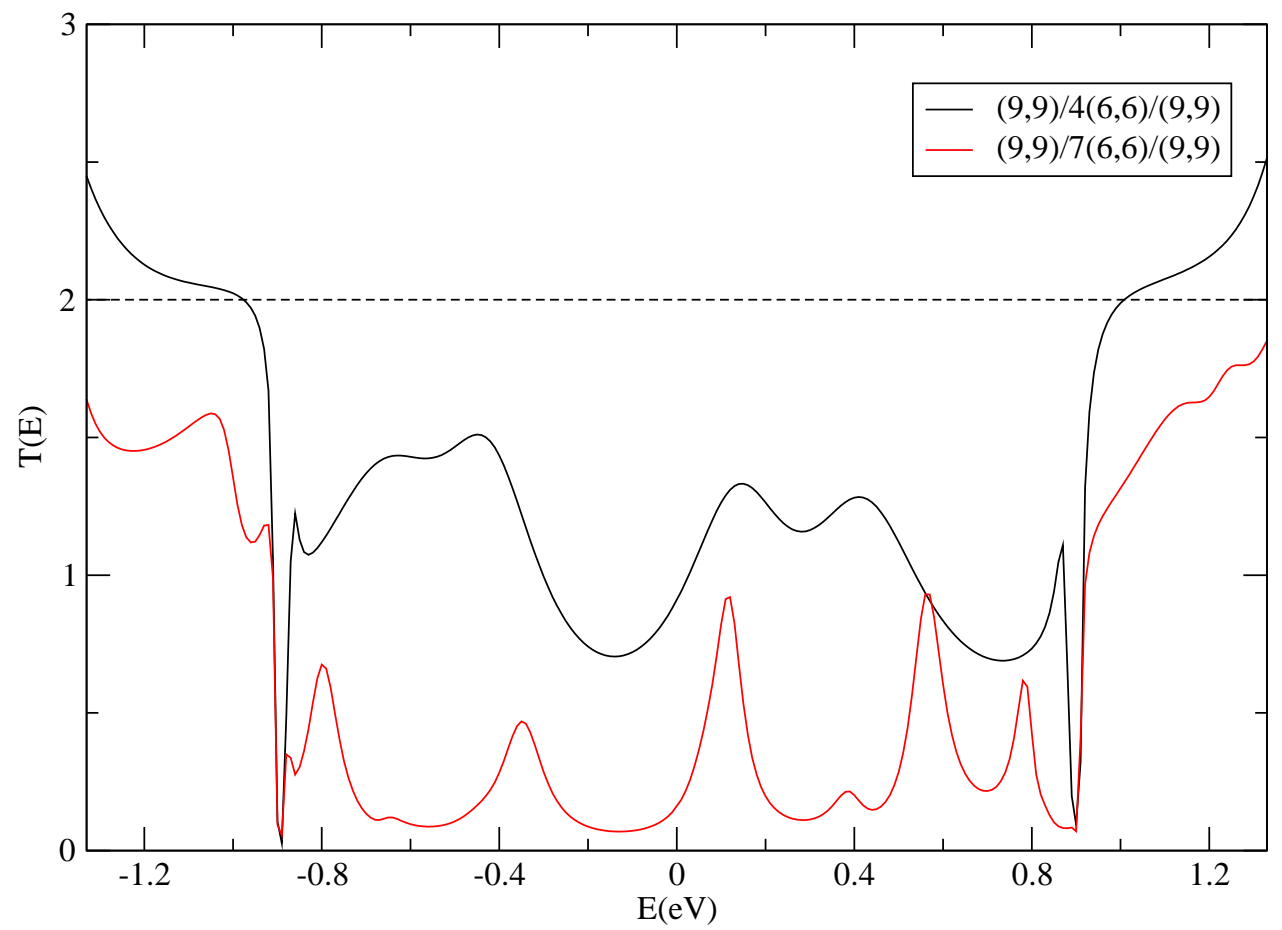

Figura 3.23: Funciones de transmisión de las estructuras $(9,9) / 4(6,6) /(9,9)$ (curva de color negro) y $(9,9) / 7(6,6) /(9,9)$ (curva de color rojo), en el rango de energías comprendido entre $-1,33 \mathrm{eV}<E<1,33 \mathrm{eV}$. Vemos que en las regiones $0,91 \mathrm{eV}<|E|<1,33 \mathrm{eV}$ la función de transmisión de la estructura $(9,9) / 7(6,6) /(9,9)$ es menor que dos debido al alargamiento de la parte central del nanotubo, lo que disminuye la transmisión por efecto túnel. Los picos se deben a la existencia de estados resonantes, lo que produce transmisión por efecto túnel resonante. 


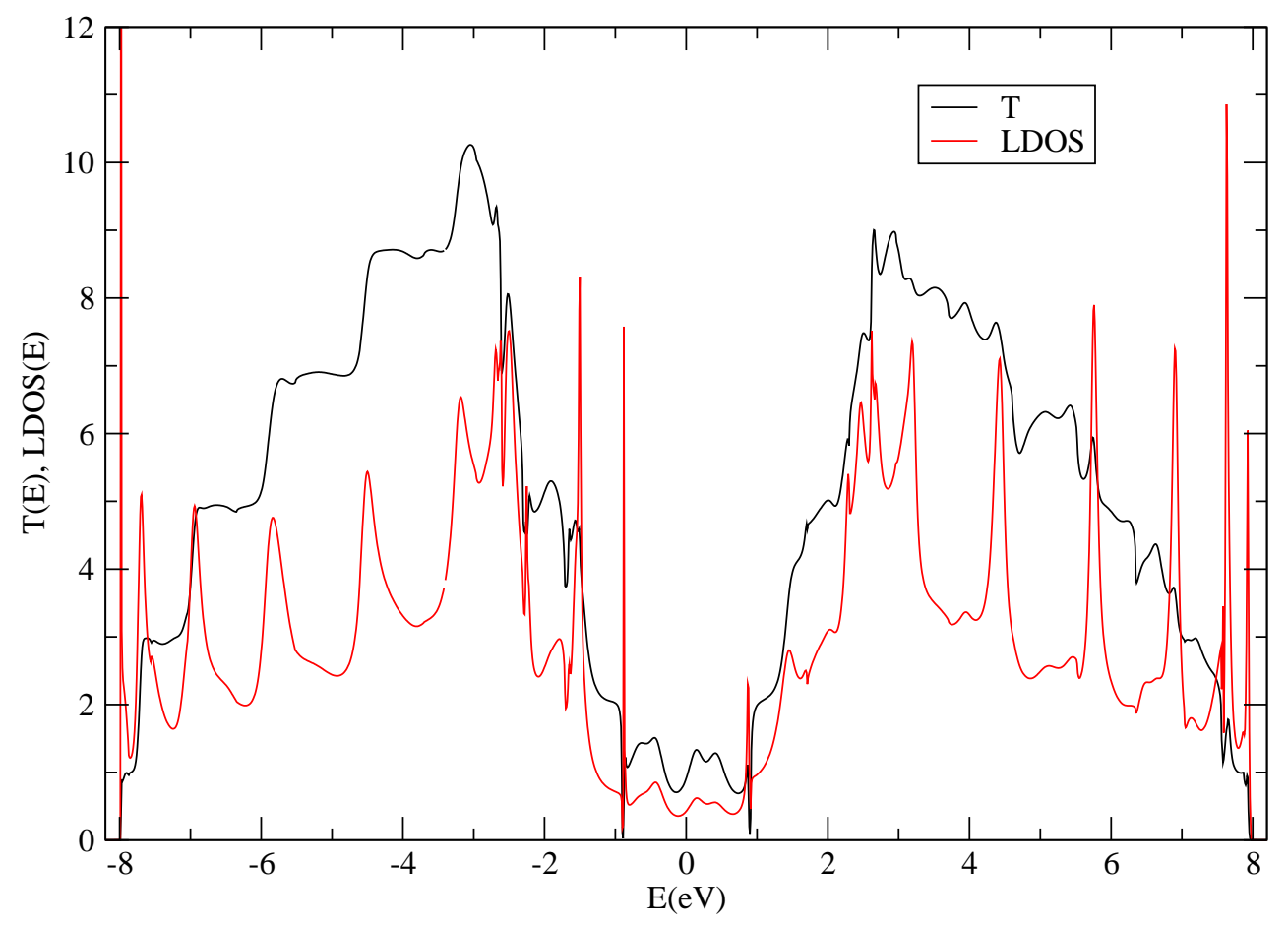

Figura 3.24: Función de transmisión de la estructura $(9,9) / 4(6,6) /(9,9)$ y densidad local de estados en la región central de dicha estructura. No se aprecia la existencia de estados localizados en dicha región, como era de esperar al tener la región central menos canales que los contactos. 
En la figura 3.23 se muestra la función de transmisión en el rango de energías $-1,33 \mathrm{eV}<E<1,33 \mathrm{eV}$ para las estructuras $(9,9) / 4(6,6) /(9,9)$ y $(9,9) / 7(6,6) /(9,9)$. Como vemos, en esta última la transmisión es menor en dicho rango, no superando en ningún caso el valor $T=2$.

Finalmente, en la figura 3.24 mostramos la función de transmisión de la estructura $(9,9) / 4(6,6) /(9,9)$ junto con la densidad local de estados en la parte central de dicha estructura (estrechamiento). Los picos de LDOS se corresponden únicamente con los bordes de la estructura de bandas del nanotubo. La densidad de estados corrobora lo dicho acerca de la inexistencia de estados localizados en el estrechamiento del nanotubo, por ser menor el número de canales en la parte central de la estructura que en los contactos.

\subsection{Acoplamiento de cavidades. Red infinita}

Analizaremos en este apartado el comportamiento de la función de transmisión cuando se realiza el acoplamiento de dos o más cavidades. En este caso es importante tener en cuenta el número de celdas unidad de los nanotubos que rodean a la cavidad, ya que las propiedades que se obtengan pueden depender de la separación entre ellas. Este dato era irrelevante en el caso de una sóla cavidad al poseer las mismas contactos semi-infinitos. Así pues, especificaremos el número de celdas unidad de los nanotubos que rodean a la cavidad utilizando la siguiente notación, para cavidades de nanotubos de tipo armchair con contactos iguales: $N(n, n) / M(m, m) / N^{\prime}(n, n)$, donde $N$ y $N^{\prime}$ indican el número de celdas unidad de los nanotubos que rodean a la cavidad, mientras que $M$ es el número de celdas unidad de la cavidad. En este trabajo siempre tendremos que $N=N^{\prime}$.

Además de estudiar en este apartado las propiedades de un sistema compuesto por varias cavidades, realizaremos el estudio de las propiedades en el límite en que tenemos infinitas cavidades iguales. Los cálculos asociados al estudio de este sistema, 

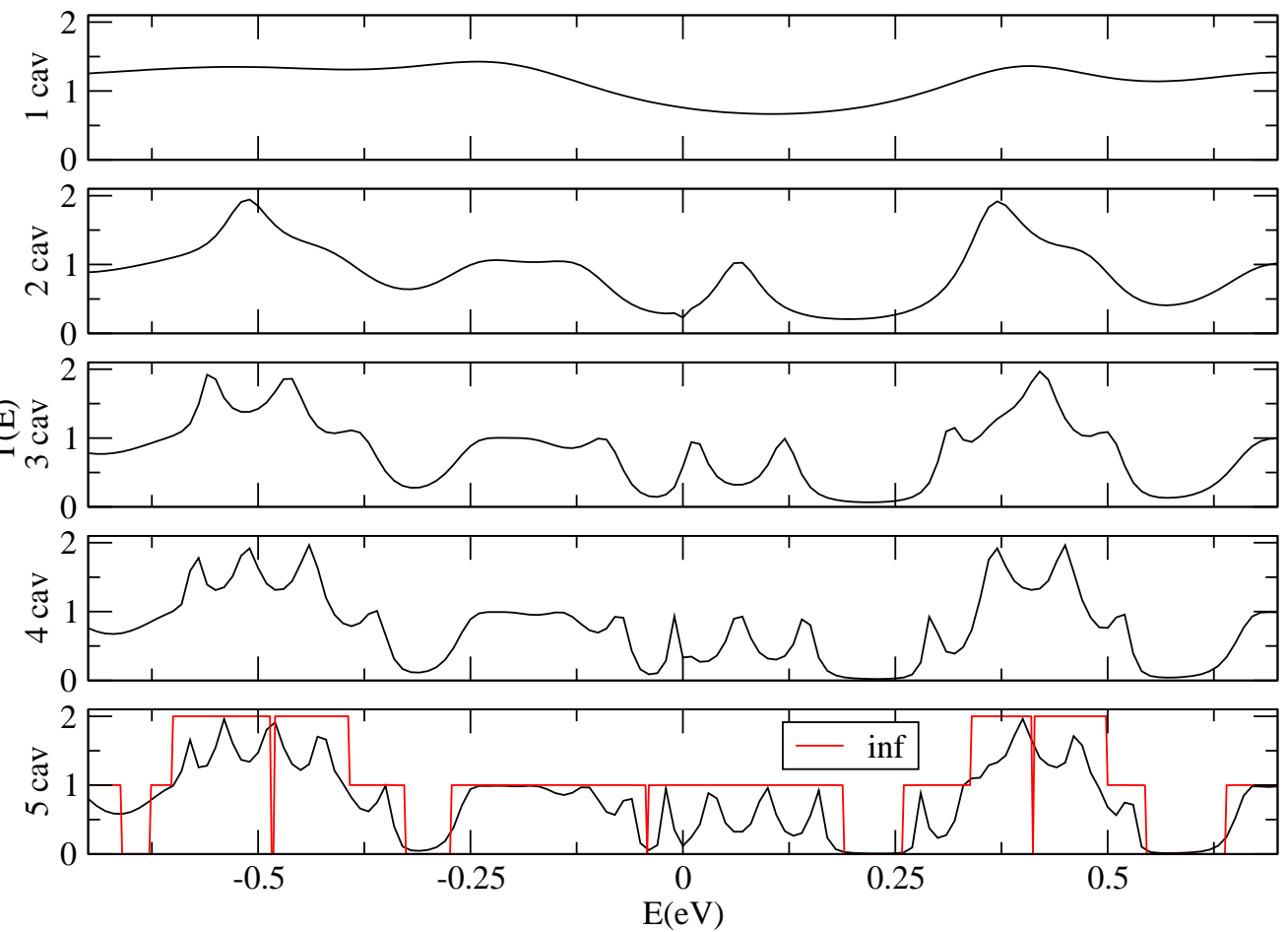

Figura 3.25: Funciones de transmisión en función de la energía, en el rango $-0,7 \mathrm{eV}<E<0,7 \mathrm{eV}$. La figura superior muestra $T$ para una sóla cavidad $4(6,6) / 4(9,9) / 4(6,6)$. Las figuras sucesivas la misma función para dos, tres, cuatro y cinco cavidades. En la figura inferior se muestra además la función de transmisión para un sistema de infinitas cavidades $4(6,6) / 4(9,9) / 4(6,6)$, en color rojo. 
que llamaremos red infinita, se llevan a cabo acoplando a la estructura con una cavidad $N(n, n) / M(m, m) / N(n, n)$, dos contactos a ambos lados que en realidad son otras dos estructuras del mismo tipo, $N(n, n) / M(m, m) / N(n, n)$. De este modo, el método recursivo descrito en el apartado 2.4 hace que los contactos se repitan de manera infinita, lo que nos lleva a un sistema compuesto por infinitas cavidades del tipo deseado.

Comenzaremos en primer lugar por el sistema $4(6,6) / 4(9,9) / 4(6,6)$. En la figura 3.25 se muestra la función de transmisión frente a la energía de un sistema compuesto por una sóla cavidad del tipo mencionado, y además para dos, tres, cuatro y cinco cavidades. Nos centramos en la región comprendida entre $-0,7 \mathrm{eV}<E<0,7 \mathrm{eV}$, donde el número de canales es el mismo para los nanotubos $(6,6)$ y $(9,9)$, por lo que podemos ver los efectos de las múltiples reflexiones en las uniones del sistema. Así mismo representamos en la gráfica inferior la transmisión para un sistema de infinitas cavidades. La simetría de este último hace que $T$ vuelva a estar cuantizada, tomando únicamente valores enteros. Es clara la evolución del sistema hacia el constituido por infinitas cavidades conforme aumentamos el número de cavidades (Jódar y Pérez-Garrido 2007).

Llama nuestra atención en la figura 3.25 la aparición de nuevas resonancias conforme aumenta el número de cavidades. Recordemos que las resonancias en la transmisión en torno al nivel de Fermi se asocian con la superposición de las ondas reflejadas en las uniones de los nanotubos. Por lo tanto, al aumentar el número de cavidades aumenta también el número de uniones y se produce un mayor número de reflexiones cuya superposición da lugar a dichas resonancias. En concreto, el número de resonancias se va incrementando en cada región de manera que es siempre igual a $n-1$, donde $n$ es el número de cavidades del sistema. Este comportamiento recuerda al sistema de Kronig-Penney para $n$ potenciales (Gasparian et al. 1997). 

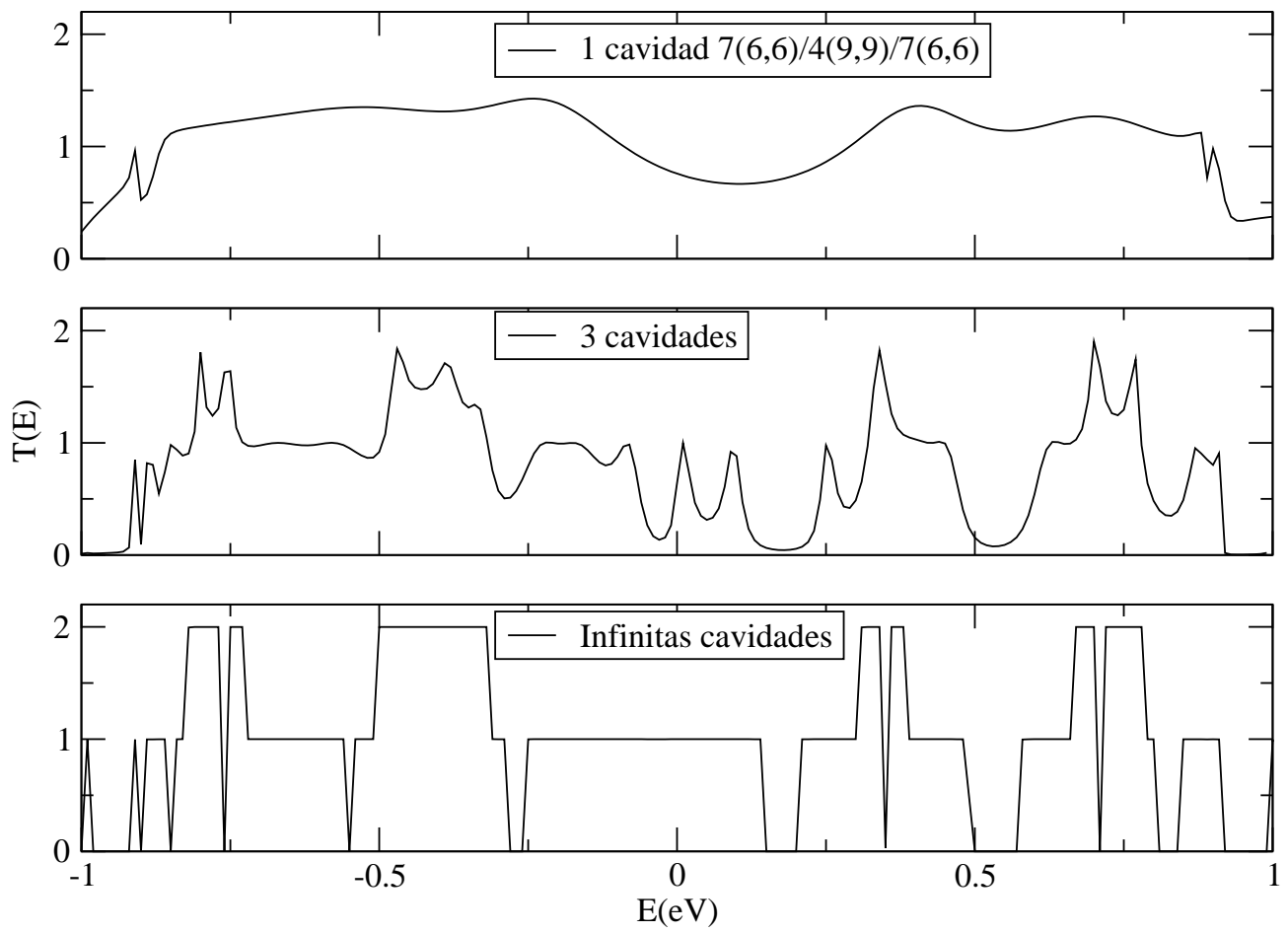

Figura 3.26: Funciones de transmisión en función de la energía, en el rango $-1 \mathrm{eV}<E<1 \mathrm{eV}$. La figura superior muestra $T$ para una sóla cavidad $7(6,6) / 4(9,9) / 7(6,6)$. Las figura central tres cavidades acopladas del mismo tipo. En la figura inferior se muestra la función de transmisión para un sistema de infinitas cavidades $7(6,6) / 4(9,9) / 7(6,6)$. 


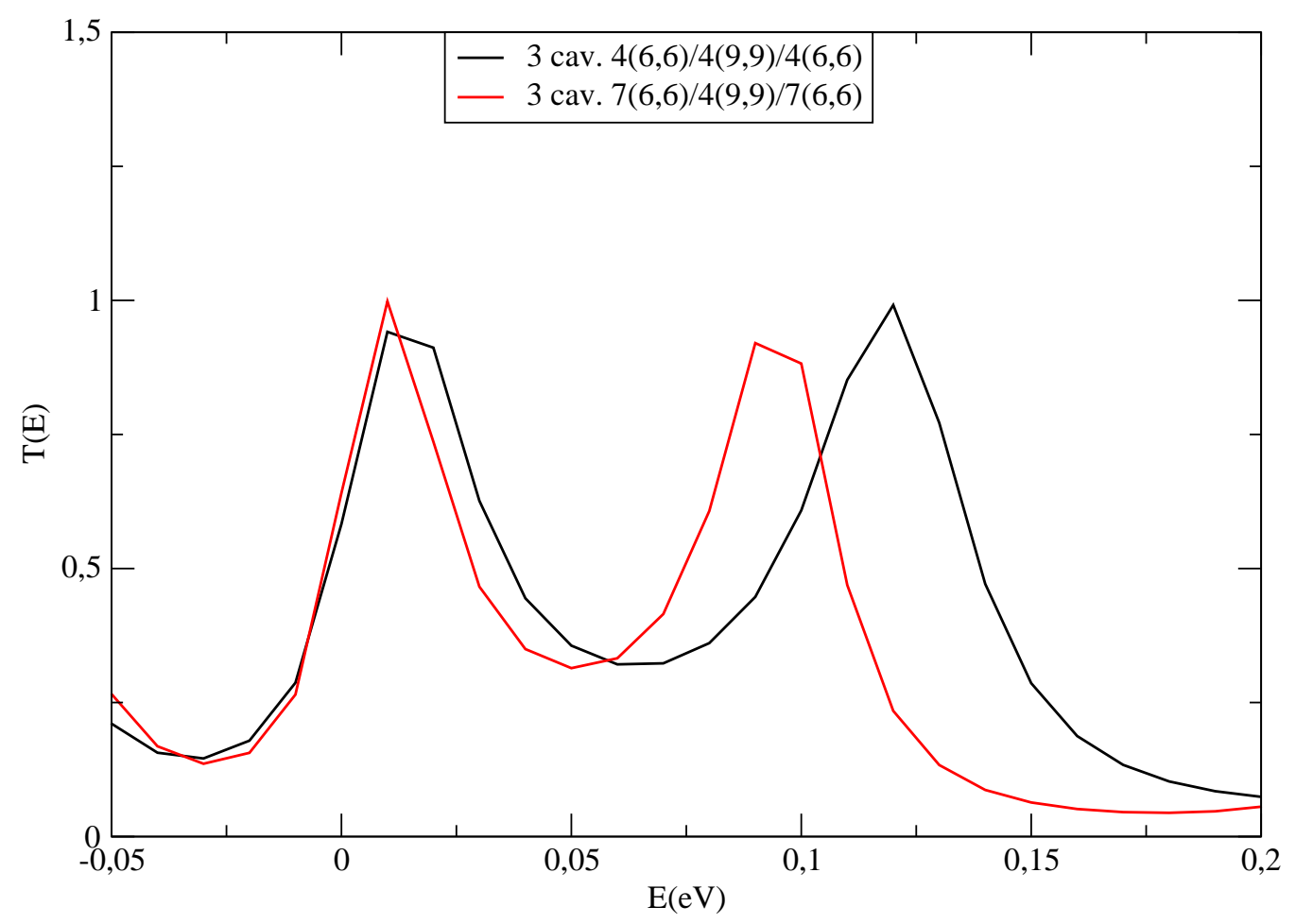

Figura 3.27: Funciones de transmisión en función de la energía, en el rango $-0,05 \mathrm{eV}<E<0,2 \mathrm{eV}$. La figura en color negro muestra $T$ para tres cavidades $4(6,6) / 4(9,9) / 4(6,6)$. La figura en color rojo tres cavidades $7(6,6) / 4(9,9) / 7(6,6)$, con mayor separación entre cavidades. Puede comprobarse que en esta última figura las dos resonancias centrales están más próximas. 
Con el fin de estudiar el efecto de la separación de las cavidades, se representa en la figura 3.26 la evolución de la transmisión de un sistema $7(6,6) / 4(9,9) / 7(6,6)$ a medida que aumenta el número de cavidades. En la parte superior de la figura, se muestra la función de transmisión para una sóla cavidad $7(6,6) / 4(9,9) / 7(6,6)$. En la central para 3 cavidades del mismo tipo, y en la inferior la función de transmisión para el límite de infinitas cavidades $7(6,6) / 4(9,9) / 7(6,6)$. Como vemos aparecen nuevas resonancias que siguen idéntica regla que en el sistema de la figura 3.25, referido al caso de 4 celdas en los contactos (cavidades más cercanas entre sí). Se pueden comparar ambas funciones en la figura 3.27, donde se muestra la función de transmisión frente a la energía de los dos sistemas mencionados para el caso en que se realiza el acoplamiento de tres cavidades. En dicha figura se aprecia un ligero acercamiento de las resonancias en el nanotubo $7(6,6) / 4(9,9) / 7(6,6)$ con respecto al $4(6,6) / 4(9,9) / 4(6,6)$. Este resultado también puede interpretarse en términos del modelo de Kronig-Penney, ya que el alejamiento de las cavidades disminuye la repulsión entre los niveles de energía y esto produce un acercamiento de los picos. En el límite de separación infinita de las cavidades los picos aparecerían como uno solo.

Finalmente, en la figura 3.28 se muestra la función de transmisión frente a la energía de los dos sistemas anteriores, $4(6,6) / 4(9,9) / 4(6,6)$ y $7(6,6) / 4(9,9) / 7(6,6)$, en el límite de infinitas cavidades, con el fin de establecer una comparación entre las mismas. Como vemos las bandas permitidas son más anchas en el caso de la estructura $4(6,6) / 4(9,9) / 4(6,6)$. Nuevamente el modelo de Kronig-Penney nos permite interpretar este hecho. En este modelo, las barreras de potencial débiles presentan regiones prohibidas más estrechas que las barreras de potencial más fuertes. En nuestro caso, cuando tenemos la estructura $4(6,6) / 4(9,9) / 4(6,6)$, tenemos el equivalente a una barrera de potencial más débil que para la estructura $7(6,6) / 4(9,9) / 7(6,6)$, ya que los electrones pueden atravesar la región $4(6,6)$ por efecto túnel con más facilidad que la región $7(6,6)$. 

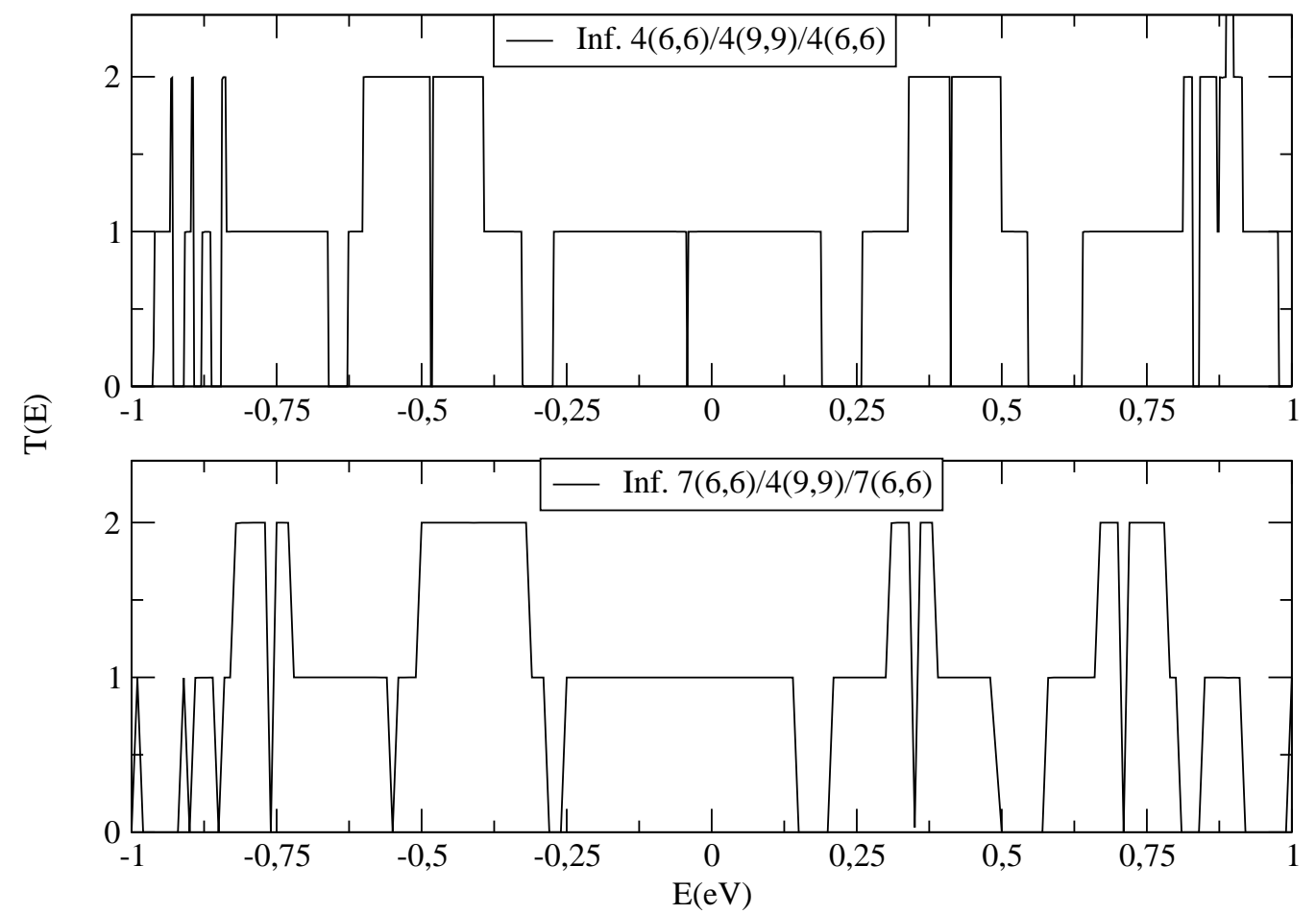

Figura 3.28: Funciones de transmisión en función de la energía, en el rango $-1 \mathrm{eV}<E<1 \mathrm{eV}$. La figura superior muestra $T$ para infinitas cavidades $4(6,6) / 4(9,9) / 4(6,6)$. La figura inferior infinitas cavidades $7(6,6) / 4(9,9) / 7(6,6)$, con mayor separación entre cavidades. Puede comprobarse que la anchura de la región central donde $T=1$ es menor en esta última figura que en la superior. 


\section{Capítulo 4}

\section{Oscilaciones de Bloch}

En los capítulos anteriores hemos desarrollado el formalismo necesario para obtener las propiedades de transmisión de los electrones libres en nanotubos, aplicando dicho formalismo al cálculo de la transmisión en sitemas con cavidades y estrechamientos. En este capítulo pretendemos estudiar la dinámica de los electrones cuando sometemos a los nanotubos a un campo eléctrico externo.

Cuando un paquete de ondas se encuentra en una cadena unidimensional de átomos sometida a un campo eléctrico, se producen movimientos ondulatorios de dicho paquete que tienen lugar a causa de las reflexiones de Bragg en los extremos de las regiones de Brillouin del sistema. Esto es válido siempre que el campo eléctrico no sea tan intenso que pueda existir cierta probabilidad de saltar a otra banda. Este fenómeno, que recibe el nombre de Oscilaciones de Bloch, fue predicho teóricamente por Zener en 1934. Sin embargo, la observación experimental del mismo no fue posible hasta la pasada década (Feldmann et al. 1992). Los recientes avances tecnológicos han hecho posible el estudio experimental de las oscilaciones de Bloch, lo que ha renovado el interés por el tema tanto a nivel teórico como experimental. Hasta donde sabemos no existen trabajos, a excepción del nuestro (Jódar et al. 2007b, Jódar et al. 2007a) que muestren este fenómeno en nanotubos de carbono. 


\subsection{Fundamento teórico}

En el estudio del movimiento de los electrones en sólidos cristalinos bajo la acción de un campo eléctrico, el tratamiento semiclásico del problema supone que el momento en la red cristalina del electrón, $\vec{p}$, satisface la ecuación:

$$
\hbar \dot{\vec{p}}=-e \vec{E},
$$

donde $\vec{E}$ es el campo eléctrico aplicado. Dada la igualdad anterior, el momento $\vec{p}$ debe aumentar por efecto del campo eléctrico hasta que se cumpla la condición de Bragg para la difracción por la red. En este caso, el electrón se refleja y cambia el sentido de su movimiento. En estas condiciones, el electrón efectúa un movimiento periódico denominado oscilación de Bloch. Esto es válido siempre que el campo eléctrico no sea muy intenso de forma que pudiera existir una probabilidad finita de realizar una transición a otra banda, como ya hemos dicho.

Los trabajos teóricos de Bloch y Zener (Bloch 1928, Zener 1934) acerca del fenómeno anterior, permiten predecir las oscilaciones espacio-temporales de electrones en potenciales periódicos sujetos a un campo eléctrico. Estas oscilaciones, que reciben el nombre de oscilaciones de Bloch, sólo se producen si la coherencia electrónica no se rompe mediante procesos de colisión (Ashcroft y Mermin 1976). En el caso de un potencial periódico unidimensional sometido a un campo eléctrico $E$, el periodo y amplitud de dichas oscilaciones vienen dados por (Hartman et al. 2004):

$$
\begin{gathered}
T_{B}=\frac{h}{e E d}, \\
A_{B}=\frac{\Delta}{e E},
\end{gathered}
$$

donde $d$ es el periodo de la red y $\Delta$ es la anchura de la banda en la que se mueve el electrón. 
Esta dinámica está alejada del movimiento acelerado del electrón sometido a una fuerza eléctrica que se esperaría de acuerdo con la física clásica. Puesto que el electrón se encuentra dentro de una red cristalina, el vector de la red recíproca $\vec{K}$ no puede tomar un valor indefinido, sino que se encuentra acotado por la anchura de banda correspondiente. Esta anchura viene dada por:

$$
\Delta=\frac{2 \pi}{d}
$$

La fuerza que actúa sobre el electrón debido al campo eléctrico se puede obtener a partir del cuasimomento del electrón $\vec{p}$. En el caso objeto de estudio, el campo eléctrico es constante y por tanto podemos escribir:

$$
\vec{F}=\frac{\vec{p}(t)-\vec{p}(0)}{t}
$$

luego:

$$
\vec{p}(t)=\vec{F} t+\vec{p}(0) .
$$

El cuasimomento $\vec{p}$ se relaciona con el vector de la red recíproca $\vec{K}$ mediante:

$$
\vec{p}=\hbar \vec{K}
$$

y de ahí que podamos escribir la ecuación (4.6) como:

$$
\vec{K}(t)=\frac{\vec{F} t}{\hbar}+\vec{K}(0) .
$$

$\mathrm{Al}$ encontrarnos en una red cristalina, los vectores $\vec{K}(t)$ y $\vec{K}(0)$ son equivalentes cuando la diferencia entre ambos es equivalente a la anchura de la banda en la que se encuentran. Esto sucede transcurrido un tiempo $T$ que podemos calcular mediante las ecuaciones (4.4) y (4.8): 


$$
\left.\begin{array}{l}
|\vec{K}(T)-\vec{K}(0)|=\Delta=\frac{2 \pi}{d} \\
|\vec{K}(T)-\vec{K}(0)|=\frac{|\vec{F}| T}{\hbar}
\end{array}\right\} \Rightarrow \quad \frac{|\vec{F}| T}{\hbar}=\frac{2 \pi}{d} .
$$

Por tanto, transcurrido un tiempo $T$ que cumpla la relación anterior, el estado del electrón vuelve a la situación inicial, esto es, el electrón está describiendo un movimiento periódico en el tiempo con un periodo $T$ igual a:

$$
T=\frac{2 \pi \hbar}{d F}=\frac{h}{e E d},
$$

que es precisamente el que adelantamos en la ecuación (4.2). Existen otras aproximaciones más sofisticadas para obtener el periodo de oscilación que conducen al mismo resultado. Así, algunos trabajos se basan en la aplicación del Teorema de la aceleración (Grecchi y Sacchetti 2001), y otros realizan una descripción del fenómeno basándose en los estados de resonancia de Wannier-Stark (Glüch et al. 2002).

La evolución de un paquete de ondas a lo largo del tiempo en un sistema con potencial periódico sometido a un campo eléctrico muestra diferentes características en función de la situación inicial del mismo. Si el paquete de ondas está inicialmente localizado alrededor de un sitio determinado de la red, dicho paquete se dispersa en el espacio regresando a su forma original tras un tiempo dado por la expresión (4.2). El centro del paquete de ondas permanece fijo. Esto implica que el paquete no se expande uniformemente sino que permanece localizado en el espacio. Este fenómeno se conoce con el nombre de localización dinámica (Nazareno et al. 1994, Nazareno y de Brito 2001). Por el contrario, si se considera un paquete de ondas que no esté inicialmente localizado, el centro del paquete sí oscila en el espacio con un periodo y amplitud dados por las ecuaciones (4.2) y (4.3), respectivamente.

La existencia de las oscilaciones de Bloch fue objeto de debate durante las décadas siguientes a su predicción teórica. El desarollo de la tecnología láser de frecuencia 
ultracorta, así como los avances en técnicas de fabricación de semiconductores, o la invención de super-redes de semiconductores, facilitó su observación experimental (Esaki y Tsu 1970). Esto se debe a que dichas redes poseen capas de materiales semiconductores que se alternan de manera que se consiguen superestructuras con grandes periodos del potencial. La probabilidad de que tengan lugar las oscilaciones en dicho material sin que se produzcan reflexiones en el mismo es mayor que en otro tipo de materiales.

Previamente a la detección de las oscilaciones de Bloch se observó su equivalente en el espacio de frecuencias: la escalera de Wannier-Stark (Wannier 1969, Mendez et al. 1988, Voisin et al. 1988). Se han observado oscilaciones de Bloch en redes de átomos de cesio ultrafrios preparados en el estado fundamental de un potencial periódico inducido ópticamente, en presencia de una excitación externa similar a un campo eléctrico constante (Dahan et al. 1996). Así mismo, se han observado fenómenos equivalentes a estas oscilaciones en sistemas de fibras ópticas (Grifoni y Hänggi 1998, Pertsch et al. 1999). Los avances experimentales han permitido desarrollar distintas técnicas para detectar estas oscilaciones en los últimos años (Feldmann et al. 1992, Leo et al. 1992, Waschke et al. 1993, Leisching et al. 1994, Dekorsy et al. 1994, Ignatov et al. 1994, Unterrainer et al. 1996).

\subsection{Método de cálculo}

Las ecuaciones teóricas dadas por las expresiones (4.2) y (4.3) se refieren a potenciales unidimensionales periódicos sometidos a un campo eléctrico unidimensional. En el caso de los nanotubos de carbono hemos de tener en cuenta que tenemos sistemas cuasi-unidimensionales con una anchura distinta de cero. En particular, los nanotubos de una sóla capa, como los que se muestran en este trabajo, se consideran como sistemas bidimensionales. Esto es debido a que el tratamiento teórico del mismo se realiza a partir de una lámina plana de grafeno enrollada sobre sí misma. 
Este hecho ha de ser contemplado en la aplicación del campo eléctrico, por lo que antes de comenzar con el desarrollo del método de cálculo debemos aclarar cómo se aplica el campo eléctrico utilizado en nuestro trabajo.

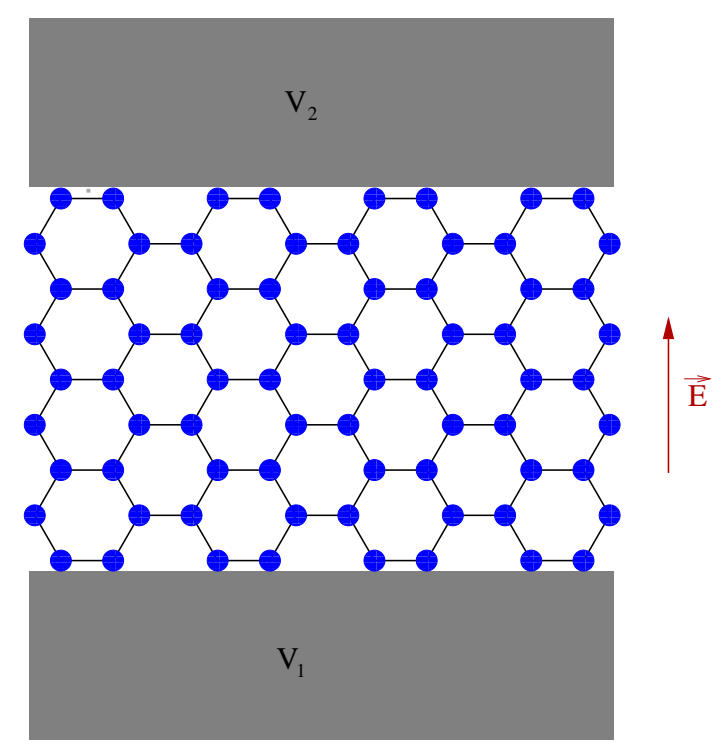

Figura 4.1: Representación esquemática del campo eléctrico aplicado sobre un nanotubo de carbono, desplegado en una capa de grafeno. El campo eléctrico tiene una única componente paralela al eje del nanotubo, que actúa a lo ancho de todo el nanotubo. El efecto es similar a conectar ambos extremos del nanotubo a dos electrodos a diferente potencial eléctrico.

En la figura 4.1 se representa gráficamente de forma esquemática el campo eléctrico aplicado sobre el nanotubo de carbono. Se trata de un campo eléctrico constante dirigido según el eje y (eje del nanotubo), y que actúa sobre todo el ancho del nanotubo. Podemos decir, por tanto, que trabajamos con un campo eléctrico unidimensional sobre un sistema bidimensional. Esta elección del campo eléctrico hace que los resultados aquí obtenidos sean factibles de ser contrastados experimentalmente conectando el nanotubo a dos electrodos a diferente potencial eléctrico.

Existen diferentes técnicas para realizar el estudio de la dinámica de las osci- 
laciones de Bloch (Hartman et al. 2004). Nosotros vamos a trabajar dentro de la aproximación del modelo tight-binding, considerando además que el hamiltoniano del sistema no depende explícitamente del tiempo. Así pues, añadimos un campo eléctrico constante $E$ unidimensional, incluyendo en los elementos diagonales del hamiltoniano del sistema la energía eléctrica correspondiente. En el modelo de orbitales $\pi$ el Hamiltoniano del sistema viene dado por:

$$
H=H_{0}+H_{t}+H_{E},
$$

donde $H_{0}$ es el término que incluye la energía de sitio de los átomos sin considerar el campo eléctrico aplicado, $H_{t}$ es el término de interacción de los átomos enlazados entre sí, y $H_{E}$ es el término que incluye la energía eléctrica de los átomos en el campo eléctrico considerado. Así, cada término viene dado por:

$$
H_{0}=\sum_{i} \epsilon_{0 i} a_{i}^{+} a_{i},
$$

donde $\epsilon_{0 i}$ es la energía del sitio $i$, que tomamos igual a cero. $a_{i}^{+}$y $a_{i}$ son los operadores creación y destrucción, respectivamente. El segundo término lo escribimos como:

$$
H_{t}=-t \sum_{i, j} a_{i}^{+} a_{j}+c . c .,
$$

donde el sumatorio sobre $i$ y $j$ se extiende únicamente sobre átomos enlazados entre sí. Tomaremos la energía de interacción $t=2,66 \mathrm{eV}$. El factor c.c. denota el complejo conjugado del término anterior.

Por último, $H_{E}$ viene dado por:

$$
H_{E}=\sum_{i} \epsilon_{i} a_{i}^{+} a_{i},
$$


y es el que introduce el campo eléctrico en el sistema. En cada sitio, la energía $\epsilon_{i}$ viene dada por:

$$
\epsilon_{i}=e y_{i} E
$$

donde $y_{i}$ representa la coordenada $y$ de cada átomo. Hemos colocado el nanotubo de manera que su eje sea paralelo al eje $y$.

En estas condiciones, podemos resolver la ecuación de Schrödinger dependiente del tiempo expandiendo la función de onda como combinación lineal de los estados estacionarios del sistema con campo eléctrico.

Supongamos que las autoenergías y autofunciones obtenidas al resolver la parte estacionaria con campo eléctrico (apartado 2.1.1, con la inclusión en el hamiltoniano de la energía eléctrica en los elementos diagonales) vienen dadas por $\epsilon_{j}(E)$ y $\left|\Psi_{j}(E)\right\rangle$, respectivamente, y que el electrón inicialmente tenga una función de onda $\psi_{0}$. La solución de la ecuación de Schrödinger dependiente del tiempo $|\Psi(E, t)\rangle$ se puede entonces escribir como:

$$
|\Psi(E, t)\rangle=\sum_{j} C_{j} e^{-i \epsilon_{j}(E) t / \hbar}\left|\Psi_{j}(E)\right\rangle,
$$

donde los coeficientes $C_{j}$ representan la amplitud de probabilidad de que el sistema se encuentre inicialmente en el estado $j$. Se calculan a través de la proyección del estado inicial $\psi_{0}$ en cada uno de los autoestados del hamiltoniano (Schwabl 2001):

$$
C_{j}=\left\langle\Psi_{j}(E) \mid \psi_{0}\right\rangle
$$

A partir de las funciones de onda dependientes del tiempo dadas por la ecuación (4.16) podemos obtener diferentes propiedades físicas que nos ayudarán en el estudio y análisis de la dinámica del sistema. Obtendremos la función de participación en función del tiempo $P_{j}(t)$ a través de la expresión (2.16), utilizando para ello la función 
de onda con campo eléctrico dependiente del tiempo $|\Psi(E, t)\rangle$. Como sabemos, la función de participación es una medida de la localización de la función de onda, por lo que su cálculo nos permitirá conocer la evolución del tamaño del paquete de ondas a lo largo del tiempo. Otra magnitud física de interés para el estudio de la dinámica de las oscilaciones de Bloch es el desplazamiento cuadrático medio $(D C M)$, que nos proporciona una estimación de la dispersión del paquete de ondas en el espacio. El desplazamiento cuadrático medio lo obtendremos a lo largo de la dirección de propagación de la onda sobre el eje del nanotubo, que supondremos paralelo al eje $y$ en un sistema cartesiano. Por tanto, lo denotaremos como $\left\langle y^{2}(t)\right\rangle$ :

$$
\left\langle y^{2}(t)\right\rangle=\sum_{i}\left|\Psi^{i}(E, t)\right|^{2}\left|y_{i}\right|^{2},
$$

donde $\Psi^{i}(E, t)$ es la amplitud de probabilidad de encontrar al electrón en el átomo correspondiente $i$.

Aunque no es objeto de este trabajo, sabemos que para tiempos infinitamente largos el desplazamiento cuadrático medio ha de tomar los valores:

$$
\left\langle y^{2}\right\rangle \sim t^{\alpha} \quad \text { donde } \begin{cases}\alpha<1 & \text { para régimen localizado } \\ \alpha=1 & \text { para régimen difusivo } \\ \alpha=2 & \text { para régimen balístico }\end{cases}
$$

El valor de la frecuencia de las oscilaciones de las magnitudes calculadas en función del tiempo, como el desplazamiento cuadrático medio, hemos de contrastarlo con la ecuación teórica del periodo dada por (4.2). Para obtener de manera precisa dicha frecuencia realizaremos el cálculo de la transformada de Fourier de dichas funciones. La transformada de Fourier es una herramienta matemática que permite analizar una señal periódica en términos de su contenido frecuencial o espectro. En la siguiente subsección hacemos un breve repaso de la transformada de Fourier, e introducimos la transformada rápida de Fourier, FFT, empleada para obtener 
numéricamente las frecuencias de oscilación.

\subsubsection{La transformada de Fourier}

En matemática, la transformada de Fourier es una aplicación que hace corresponder a una función $f$ con valores complejos y definida en la recta, otra función $g$ definida de la manera siguiente:

$$
g(\xi)=\frac{1}{\sqrt{2 \pi}} \int_{x=-\infty}^{x=\infty} f(x) e^{-i \xi x} d x .
$$

La transformada de Fourier se utiliza para pasar al dominio de la frecuencia, lo que nos permite en muchas situaciones obtener información que no es evidente en el dominio del tiempo.

En nuestro caso, la información viene del resultado de cálculos numéricos y por lo tanto no disponemos de funciones continuas. Tenemos entonces que realizar el cálculo de lo que se conoce como transformada de Fourier discreta, DFT (Discrete Fourier Transform). En ambas transformadas se realiza el desarrollo de la función que se quiere analizar mediante series de Fourier, que describen señales periódicas como combinaciones de señales sinusoidales. De este modo podemos establecer una dualidad entre tiempo y frecuencia, de forma que operaciones realizadas en el dominio temporal tengan su dual en el dominio de frecuencias. Así, la transformada de Fourier es ampliamente utilizada en tratamiento de señales y en campos afines para analizar las frecuencias presentes en una señal muestreada, resolver ecuaciones diferenciales parciales y realizar otras operaciones, como convoluciones. La transformada de Fourier discreta puede calcularse de modo muy eficiente mediante un algoritmo llamado FFT (Fast Fourier Transform, esto es, transformada rápida de Fourier). Si realizáramos el cálculo de la DFT directamente, tendríamos una complejidad computacional que crece con $N^{2}$, siendo $N$ el tamaño de los datos de entrada. Esto quiere decir que el número de operaciones aritméticas a realizar es proporcio- 
nal a $N^{2}$ y por lo tanto también lo es el tiempo necesario para llevar a cabo dicho cálculo. El algoritmo FFT reduce esta complejidad a $N \log N$.

En este trabajo, obtenemos el espectro de frecuencias de las funciones periódicas asociadas al movimiento de los electrones en el nanotubo sometido a un campo eléctrico. En particular, calcularemos las frecuencias representativas del desplazamiento cuadrático medio del electrón y de su función de participación. El cálculo numérico de la frecuencia de dichas funciones a través de la transformada de Fourier nos permitirá contrastar los resultados obtenidos con la expresión teórica del periodo de Bloch (ecuación (4.2)).

\subsection{Oscilaciones de Bloch en nanotubos puros. On- das de Wannier}

En este apartado estudiaremos la dinámica de funciones de onda iniciales de Wannier (completamente localizadas en un sitio de la red) en nanotubos puros sometidos a un campo eléctrico constante. Para ello utilizaremos la expresión (4.16) tomando como estado inicial ondas localizadas en un sólo átomo. De este modo podremos observar el fenómeno anteriormente descrito de la localización dinámica.

\subsubsection{Funciones de onda. Probabilidad de ocupación}

Aplicaremos nuestro estudio en primer lugar a la obtención de las funciones de onda dependientes del tiempo para un nanotubo $(6,6)$. A través del cálculo de las funciones de onda podemos obtener la probabilidad de encontrar al electrón en una determinada coordenada $y$ del eje del nanotubo en cada instante de tiempo. 


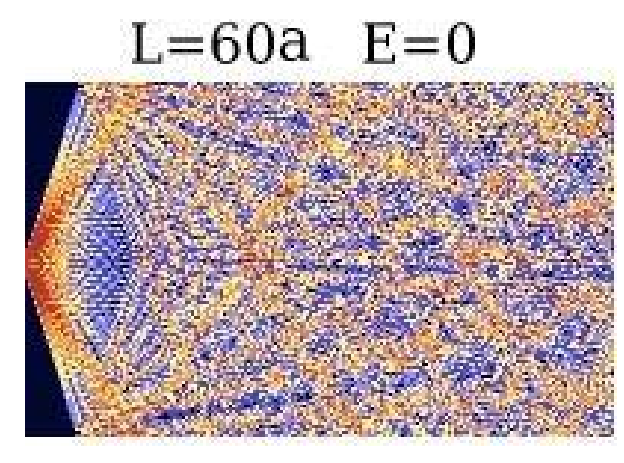

Figura 4.2: Evolución temporal sin campo eléctrico de una onda de Wannier inicialmente localizada en un átomo perteneciente al anillo central del nanotubo $(6,6)$. El eje de ordenadas muestra la probabilidad de encontrar al electrón en una determinada coordenada $y$ del eje del nanotubo, de longitud $L=60 a$. El eje de abscisas representa el tiempo, en una escala que va desde $t=0$ hasta $t=12 \cdot 10^{-14} \mathrm{~s}$. Los tonos rojizos se corresponden con valores elevados de la probabilidad, los tonos azules con valores pequeños, y los amarillos con valores intermedios. Puede verse cómo la onda evoluciona difundiéndose hasta extenderse a lo largo de todo el nanotubo.

Los resultados obtenidos en nuestro cálculo se pueden analizar gráficamente con facilidad representando el valor de la probabilidad mediante un código de colores. Representaremos en el eje de ordenadas la coordenada $y$ (eje del nanotubo), y en el de abscisas el tiempo. Los valores pequeños de la probabilidad se corresponden con los tonos azules, mientras que los valores grandes se representan a través de los tonos naranjas y rojos. Los tonos amarillos se corresponden con valores intermedios de la probabilidad. Veamos en primer lugar la figura 4.2. En ella se muestra la evolución temporal de la probabilidad de encontrar al electrón en una determinada coordenada $y$ del eje del nanotubo para una onda inicialmente localizada sobre la que no actúa campo eléctrico alguno. En este caso la onda se expande uniformemente a lo largo de todo el nanotubo, como se aprecia claramente en dicha figura. 
Al aplicar un campo eléctrico, las funciones de onda comienzan a oscilar, como podemos ver en la figura 4.3, donde se representan las probabilidades de encontrar al electrón en una determinada coordenada $y$ del eje del nanotubo en función del tiempo para funciones de onda iniciales de Wannier. Los cálculos son para un nanotubo $(6,6)$ sometido a diversos valores del campo eléctrico. En el nanotubo $(6,6)$ la longitud de una única celda unidad, según la expresión (1.15) viene dado por $L=a$, donde recordemos que denotamos por a la constante de red para el grafito bidimensional, de valor $a=2,46 \AA$. Así pues, la longitud total para un nanotubo de 60 celdas es $L=60 a=14,76 \mathrm{~nm}$. La figura 4.3 a) muestra los resultados obtenidos para $E=271 \mathrm{MV} / \mathrm{m}$, la b) para $E=542 \mathrm{MV} / \mathrm{m}$, la c) para $E=813 \mathrm{MV} / \mathrm{m}$ y la d) para $E=1084 \mathrm{MV} / \mathrm{m}$. Usamos estos valores de $E$ tan arbitrarios porque en realidad lo que ajustamos en nuestro cálculo es la diferencia de potencial entre los extremos del nanotubo. Aunque para poder comparar las oscilaciones en nanotubos de diferente tamaño es mejor tener presentes los valores del campo eléctrico.

Como vemos en la figura 4.3, se aprecia claramente el fenómeno de la localización dinámica. Sin embargo, los efectos de borde impiden distinguir de forma precisa dichas oscilaciones para campos eléctricos pequeños. Esto es debido a que la longitud finita de nuestro sistema provoca reflexiones en los extremos del mismo que perturban la evolución temporal. Aumentando el valor del campo eléctrico podemos minimizar estos efectos, ya que aumenta la frecuencia de oscilación del paquete de ondas y disminuye su amplitud, por lo que llega al extremo del nanotubo una porción menor del paquete de ondas. De manera evidente, suprimimos los efectos de borde aumentando el tamaño del nanotubo, pero esto también hace aumentar el tiempo de computación. En este trabajo usamos, por lo tanto, campos eléctricos muy grandes. Sin embargo, todos los resultados son extrapolables a dispositivos experimentales con campos más pequeños pero nanotubos mucho más largos. Recordemos que se pueden fabricar nanotubos de hasta unos pocos milímetros de longitud. 

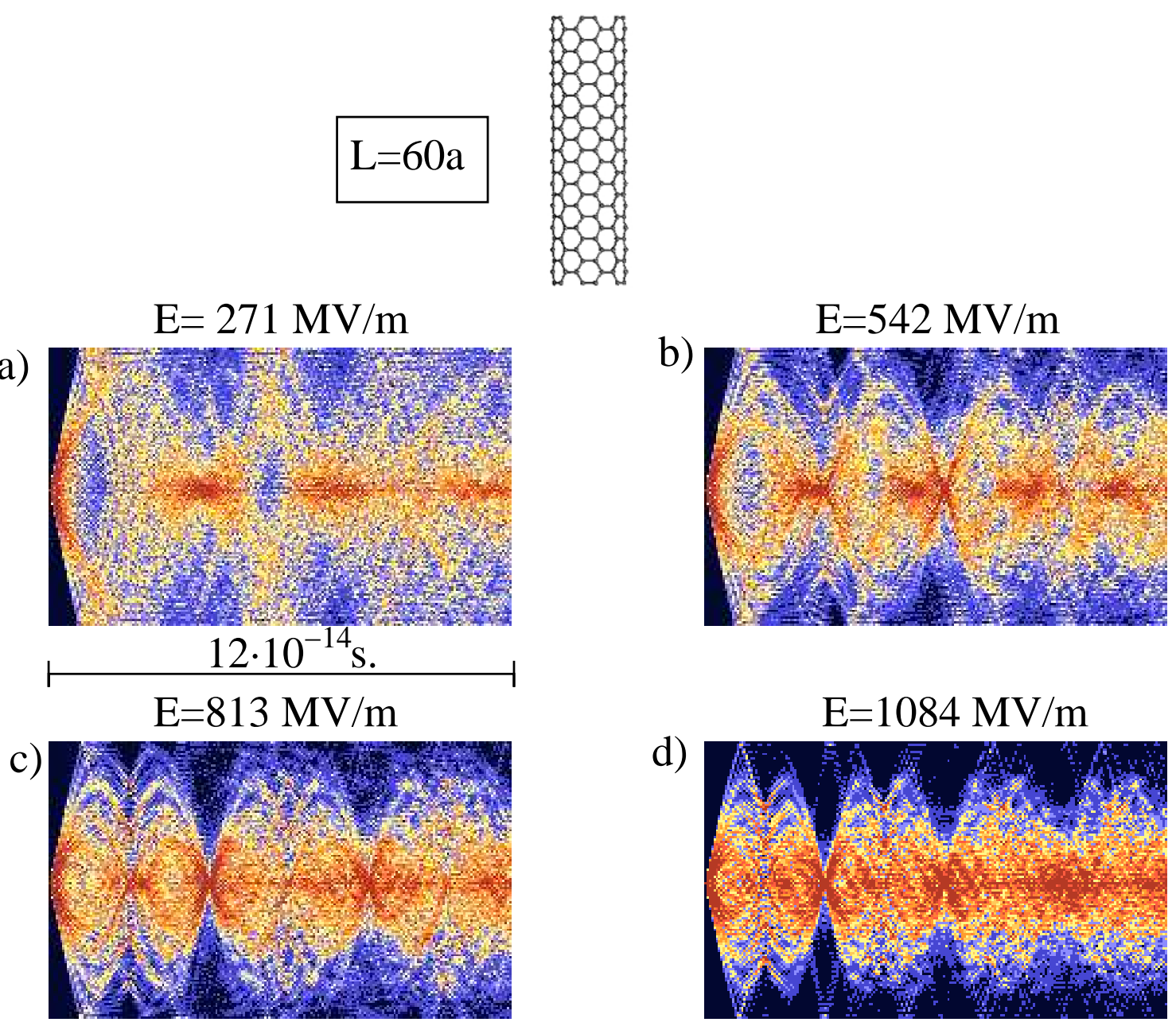

Figura 4.3: Representación de la probabilidad de encontrar al electrón en una determinada coordenada $y$ del eje del nanotubo para una función de onda inical de Wannier en función del tiempo. Los resultados que se muestran son para un nanotubo $(6,6)$ de longitud $L=60 a(a=2,46 \AA)$ a lo largo de un intervalo de tiempo que va desde $t=0$ hasta $t=12 \cdot 10^{-14} \mathrm{~s}$, para diferentes valores del campo eléctrico. La figura a) muestra los resultados obtenidos para $E=271 \mathrm{MV} / \mathrm{m}$, la b) para $E=542 \mathrm{MV} / \mathrm{m}$, la c) para $E=813 \mathrm{MV} / \mathrm{m}$ y la d) para $E=1084 \mathrm{MV} / \mathrm{m}$. A medida que aumenta el valor del campo eléctrico, las oscilaciones se hacen más claras debido a que los extremos abiertos del nanotubo afectan menos. 
$\mathrm{E}=1355 \mathrm{MV} / \mathrm{m}$

a)

c)
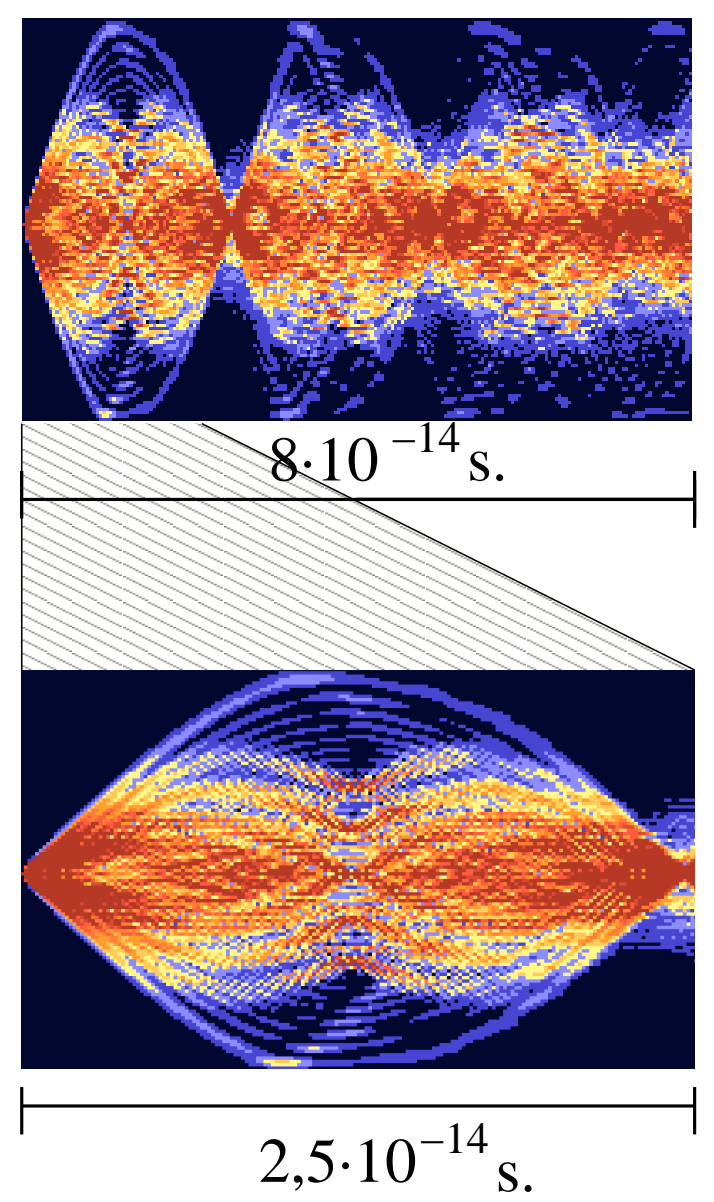

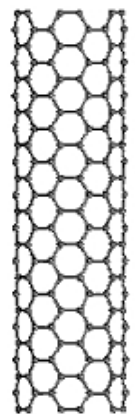

b)

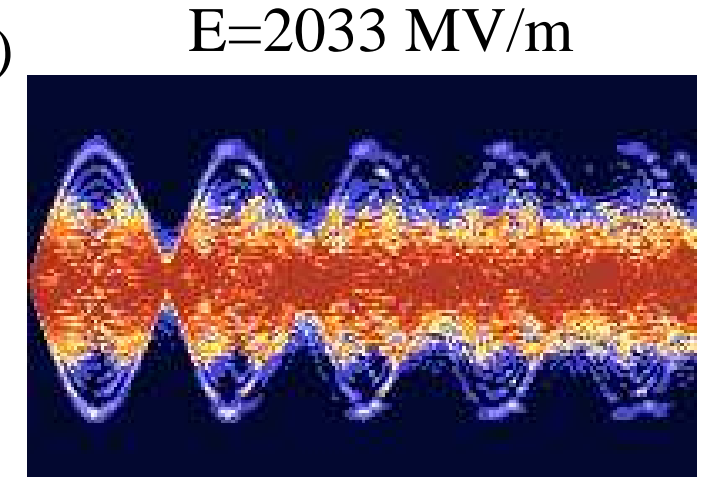

Figura 4.4: Representación de la probabilidad de encontrar al electrón en una determinada coordenada $y$ del eje del nanotubo para una función de onda inicial de Wannier en función del tiempo. Los resultados son para un nanotubo $(6,6)$ de longitud $L=60 a$. La figura a) muestra los resultados obtenidos para $E=1355 \mathrm{MV} / \mathrm{m}$ y la b) para $E=2033 \mathrm{MV} / \mathrm{m}$. El intervalo de tiempo en estas dos figuras va desde $t=0$ hasta $t=8 \cdot 10^{-14} \mathrm{~s}$. La figura c) es una ampliación de la a) para un intervalo de tiempo $0<t<2,5 \cdot 10^{-14} \mathrm{~s}$. En esta tercera figura se aprecia cómo lo que parece una oscilación está compuesta a su vez por dos oscilaciones de diferente frecuencia. 
En la figura 4.4 se representa el valor de la probabilidad de encontrar al electrón en una determinada coordenada $y$ del eje del nanotubo en función del tiempo en una onda inicial de Wannier, para los valores del campo eléctrico: a) $E=1355$ $\mathrm{MV} / \mathrm{m}$ y b) $E=2033 \mathrm{MV} / \mathrm{m}$, para un nanotubo de 60 celdas. En este caso los valores del campo eléctrico son los suficientemente altos como para que los efectos de borde no afecten demasiado a los resultados, como puede comprobarse en dicha figura. Se observa además que la oscilación presenta dos frecuencias significativas. En la figura c) se muestra lo que parece ser a simple vista una oscilación para $E=1355 \mathrm{MV} / \mathrm{m}$. Se aprecia claramente en esta figura que dicha oscilación se compone, en realidad, de dos oscilaciones. Este hecho habrá de tenerse en cuenta a la hora de obtener la frecuencia de la oscilación mediante el cálculo de la Transformada de Fourier, ya que dicha transformada arrojará dos frecuencias de oscilación. Más adelante analizaremos de este fenómeno, que guarda relación con el hecho de que la celda unidad del nanotubo tipo armchair se componga de dos filas de átomos.

Ya hemos visto en la figura 4.3 que los efectos de borde afectan a la oscilación de la onda en presencia del campo eléctrico, siendo necesario introducir campos relativamente altos para que dichos efectos afecten lo menos posible a los resultados. Si aumentamos la longitud del nanotubo podemos conseguir disminuir los efectos de borde con campos eléctricos más pequeños. En la figura 4.5 se muestran los resultados obtenidos para un $\operatorname{CNT}(6,6)$ de longitud $L=90 a=22,14 \mathrm{~nm}$, para los mismos valores del campo eléctrico que en la figura 4.3. En a) se muestran los resultados para $E=271 \mathrm{MV} / \mathrm{m}$, en b) para $E=542 \mathrm{MV} / \mathrm{m}$, en c) para $E=813$ $\mathrm{MV} / \mathrm{m}$ y en d) para $E=1084 \mathrm{MV} / \mathrm{m}$. Si bien en el caso del nanotubo de 60 celdas estos campos eléctricos eran insuficientes como para despreciar los efectos de borde, en el caso de uno de 90 celdas podemos considerar que a partir de $E=1084 \mathrm{MV} / \mathrm{m}$ dichos efectos son despreciables, como se aprecia en la figura $4.5 \mathrm{~d}$ ). Considerando un nanotubo más largo podríamos tener oscilaciones de Bloch bien diferenciadas para campos eléctricos más manejables. 
a)

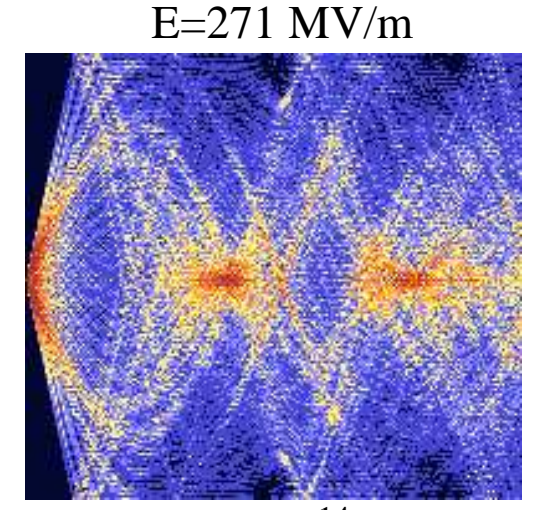

$12 \cdot 10^{-14} \mathrm{~s}$.

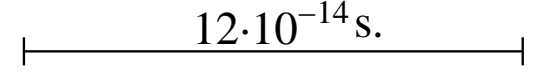

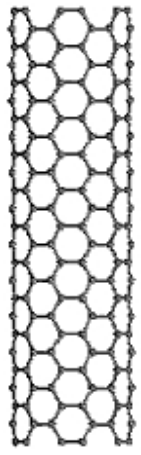

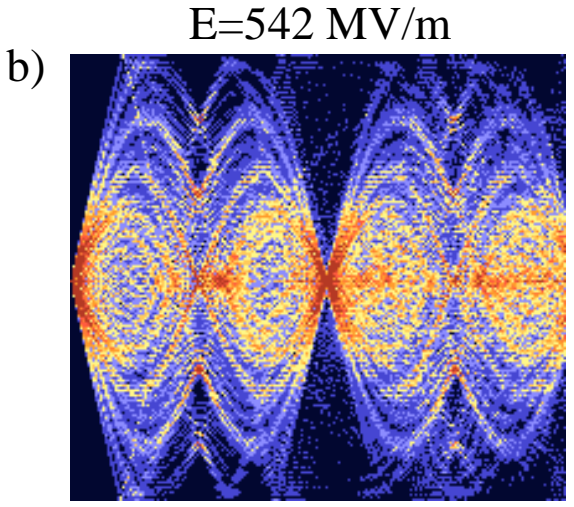

$$
\mathrm{E}=813 \mathrm{MV} / \mathrm{m}
$$

c)

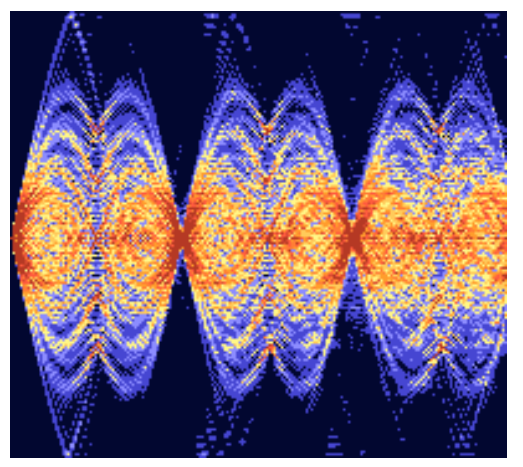

$$
\mathrm{E}=1084 \mathrm{MV} / \mathrm{m}
$$

d)

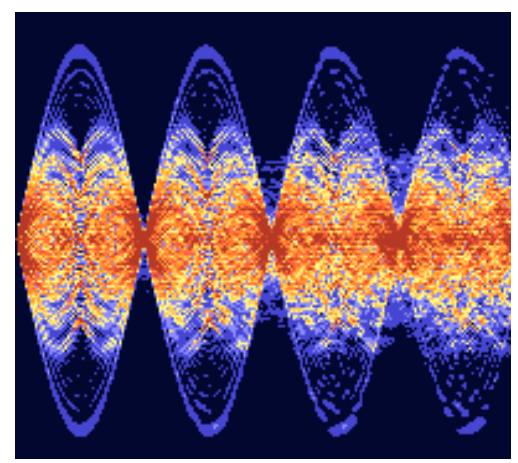

Figura 4.5: Representación de la probabilidad de encontrar al electrón en una determinada coordenada $y$ del eje del nanotubo en función del tiempo (coordenada horizontal) para una onda de Wannier inicial. Los resultados que se muestran son para un nanotubo $(6,6)$ de longitud $L=90 a$ a lo largo de un intervalo de tiempo que va desde $t=0$ hasta $t=12 \cdot 10^{-14} \mathrm{~s}$, y para diferentes valores del campo eléctrico. La figura a) muestra los resultados obtenidos para $E=271 \mathrm{MV} / \mathrm{m}$, la b) para $E=542 \mathrm{MV} / \mathrm{m}$, la c) para $E=813 \mathrm{MV} / \mathrm{m}$ y la d) para $E=1084 \mathrm{MV} / \mathrm{m}$. Los valores son los mismos que se utilizaron en el caso del nanotubo de longitud $L=60 a$, si bien en este caso las oscilaciones resultan más claras. Puede apreciarse cómo la oscilación aumenta su frecuencia y disminuye su amplitud a medida que el campo eléctrico aumenta. 


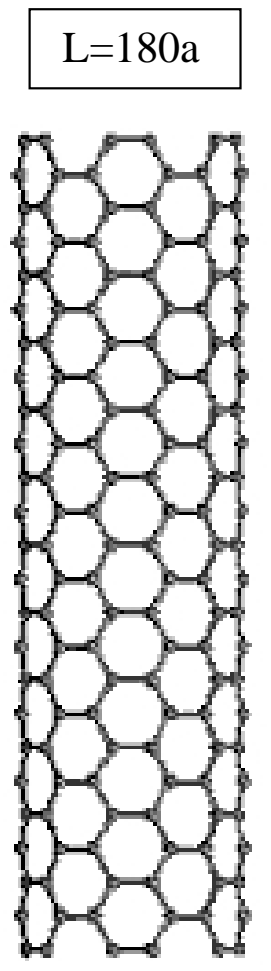

a)

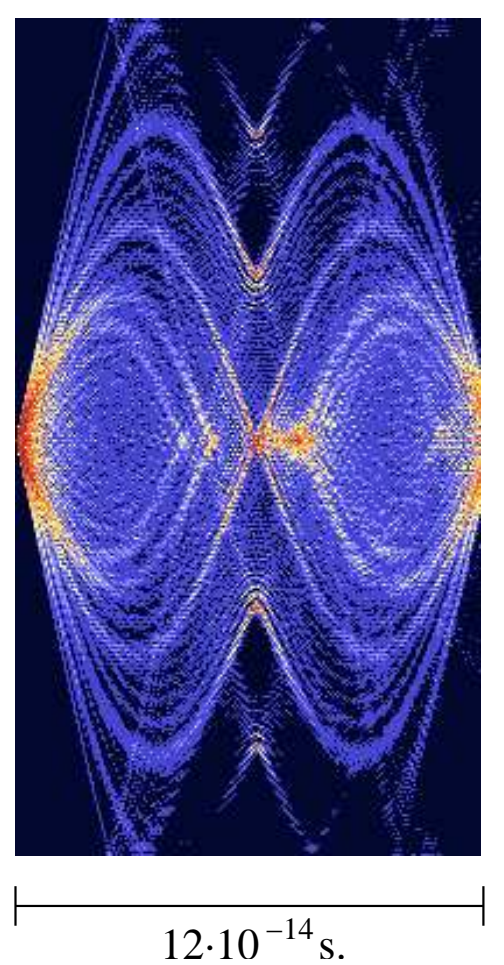

b)
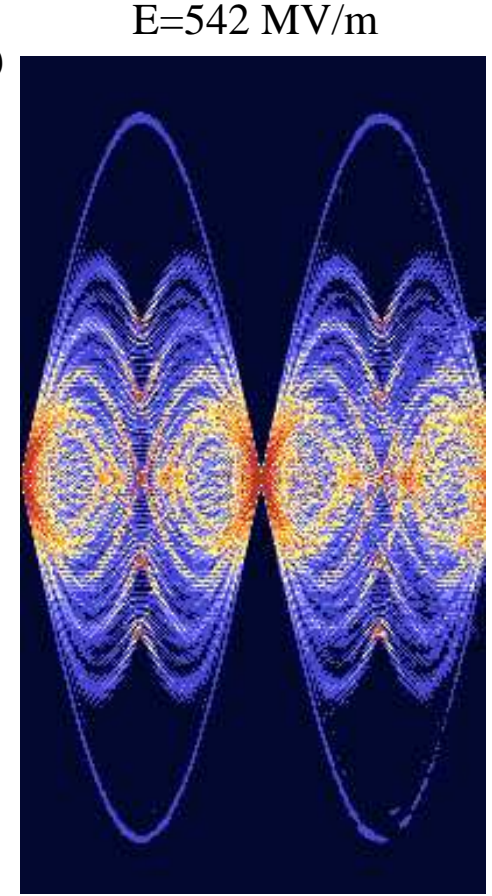

Figura 4.6: Probabilidad de encontrar al electrón en una determinada coordenada $y$ del eje del nanotubo en función del tiempo para una onda inicial de Wannier. Los resultados que se muestran son para un nanotubo $(6,6)$ de longitud $L=180 \mathrm{a}$. los valores del campo eléctrico son $E=271 \mathrm{MV} / \mathrm{m}$ para la figura a) y $E=542 \mathrm{MV} / \mathrm{m}$ para la b). El intervalo de tiempo mostrado va desde $t=0$ hasta $t=12 \cdot 10^{-14} \mathrm{~s}$. 


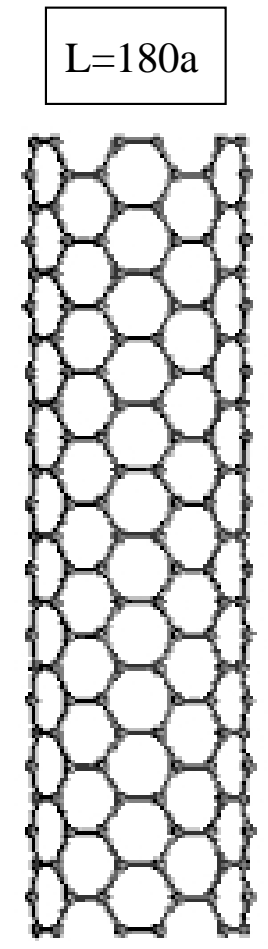

a)

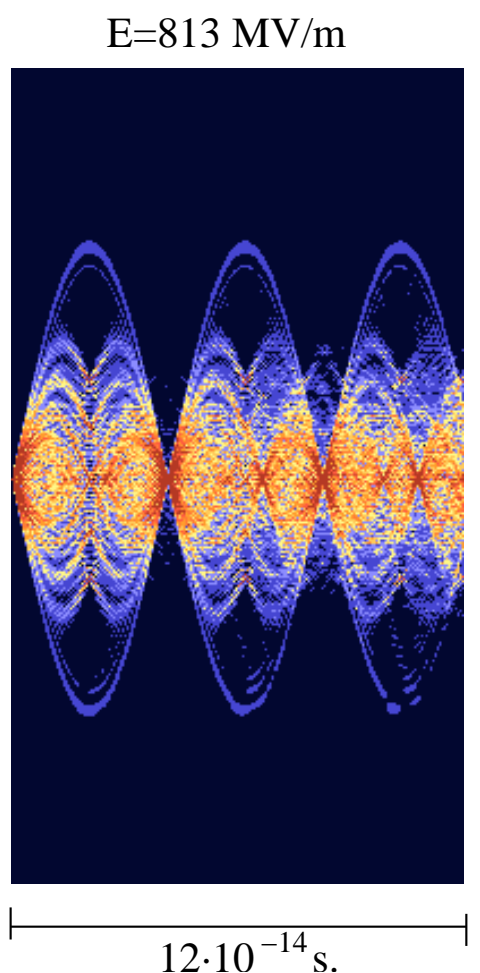

b)

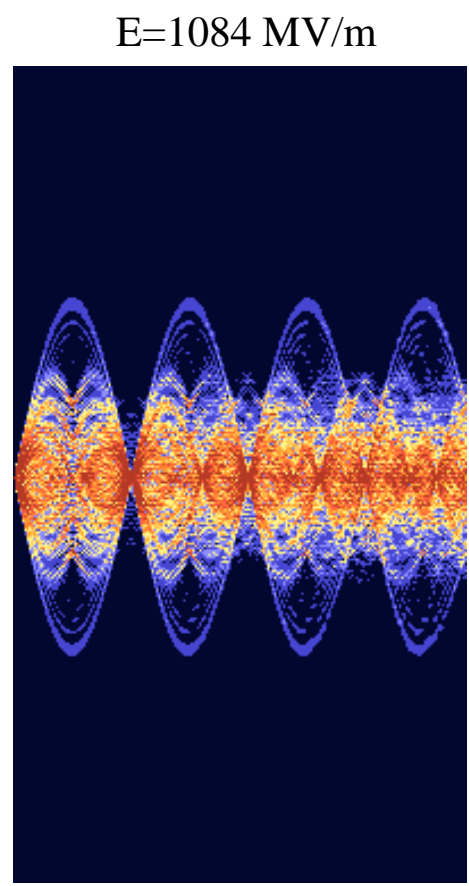

Figura 4.7: Probabilidad de encontrar al electrón en una determinada coordenada $y$ del eje del nanotubo en función del tiempo para una onda inicial de Wannier. Los resultados que se muestran son para un nanotubo $(6,6)$ de longitud $L=180 a$. Los valores del campo eléctrico son $E=813 \mathrm{MV} / \mathrm{m}$ en la figura a) y $E=1084 \mathrm{MV} / \mathrm{m}$ en la b). El intervalo de tiempo mostrado va desde $t=0$ hasta $t=12 \cdot 10^{-14}$ s. Los efectos de borde en ambos casos tienen una influencia pequeña sobre las oscilaciones. 
Una vez comprobada la influencia de la longitud del nanotubo en la obtención numérica de las oscilaciones de Bloch, vamos a extraer a continuación resultados en nanotubos con tamaños y campos eléctricos aplicados en los que los efectos de tamaño finito sean mínimos, y dentro de nuestras posibilidades computacionales.

Hemos realizado los cálculos para un nanotubo de longitud $L=180 a(\approx 45 \mathrm{~nm})$ introduciendo los mismos valores del campo eléctrico que los utilizados en las figuras 4.3 y 4.5. Los resultados se muestran en la figura 4.6, donde los valores del campo eléctrico son $E=271 \mathrm{MV} / \mathrm{m}$ para la figura a) y $E=542 \mathrm{MV} / \mathrm{m}$ para la b), y en la figura 4.7 donde $E=813 \mathrm{MV} / \mathrm{m}$ en la figura a) y $E=1084 \mathrm{MV} / \mathrm{m}$ en la b). Como vemos, en este caso podemos considerar que los resultados no se ven afectados por los efectos de borde a partir de un valor del campo eléctrico igual a $E=542 \mathrm{MV} / \mathrm{m}$.

Para finalizar este apartado mostramos en la figura 4.8 una comparación de las oscilaciones producidas en nanotubos de diferente tamaño para idéntico campo eléctrico. En esta figura vemos de nuevo, pero juntas en una misma gráfica para compararlas mejor, la probabilidad de encontrar al electrón en una determinada coordenada $y$ del eje del nanotubo en función del tiempo para un campo eléctrico igual a $E=542 \mathrm{MV} / \mathrm{m}$. Se muestran los resultados para $L=60 a$ en a), para $L=90 a$ en b), y para $L=180 a$ en c).

\subsubsection{Análisis de la oscilación}

Como hemos visto en el apartado anterior, las oscilaciones de la función de onda producidas en nanotubos de carbono a causa de un campo eléctrico unidimensional paralelo al eje del nanotubo poseen dos frecuencias de oscilación principales. Este hecho ha de ser analizado más en profundidad, ya que las ecuaciones teóricas sólo predicen una frecuencia de oscilación en sistemas sujetos a un potencial periódico unidimensional cuando dicho sistema se encuentra sometido a un campo eléctrico constante. Si en lugar de un sistema unidimensional trabajamos con un 


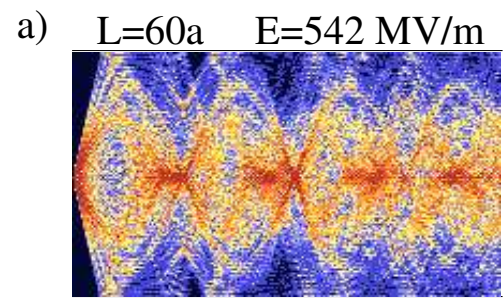

b) $\mathrm{L}=90 \mathrm{a} \quad \mathrm{E}=542 \mathrm{MV} / \mathrm{m}$

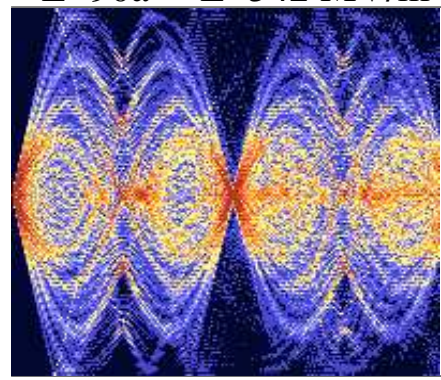

c) $\mathrm{L}=180 \mathrm{a} \quad \mathrm{E}=542 \mathrm{MV} / \mathrm{m}$

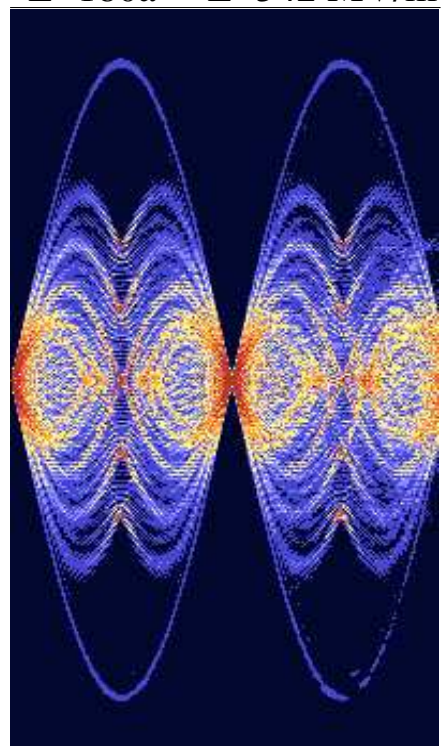

Figura 4.8: Comparación de los resultados obtenidos para la probabilidad de encontrar al electrón en una determinada coordenada $y$ del eje del nanotubo para funciones de onda iniciales de Wannier en nanotubos de diferentes longitudes, sometidos a un campo eléctrico $E=542 \mathrm{MV} / \mathrm{m}$. En la figura a) se muestran los resultados para $L=60 a$, en la b) para $L=90 a$ y en la c) para $L=180 a$. El intervalo de tiempo va desde $t=0$ hasta $t=12 \cdot 10^{-14} \mathrm{~s}$. 
sistema en dos dimensiones con un potencial eléctrico también bidimensional, la frecuencia de la oscilación será el mínimo común múltiplo de las frecuencias teóricas en las dos direcciones dadas por las componentes del campo eléctrico aplicado (Witthaut et al. 2004). Sin embargo, aunque en nuestro caso el potencial eléctrico aplicado sobre el nanotubo es unidimensional, ya que su valor depende únicamente de la coordenada $y$ del nanotubo, hemos de tener en cuenta el hecho de que dicho nanotubo es, en realidad, un sistema bidimensional.

Si la causa de la doble frecuencia obtenida se debiera a los efectos provocados por la anchura del nanotubo, éstos se pondrían de manifiesto al comparar los resultados obtenidos con nanotubos de diferente diámetro. En la figura 4.9 hemos representado la probabilidad de encontrar al electrón en una determinada coordenada y del eje del nanotubo en función del tiempo para dos nanotubos de diferente diámetro pero idéntica longitud: $(6,6)$ y $(9,9)$, de 60 celdas de longitud cada uno. El diámetro del nanotubo $(6,6)$ es igual a $3,3 a$ según la ecuación (1.9), mientras que el del $(9,9)$ es igual a 5,0a. El campo eléctrico utilizado es $E=813 \mathrm{MV} / \mathrm{m}$. Como puede apreciarse en la figura, la frecuencia de la oscilación es la misma para ambos nanotubos. Además, no se observan grandes diferencias entre ambas gráficas que puedan llevarnos a la conclusión de que la doble oscilación producida se deba a la anchura del nanotubo.

Lo que hemos venido observando en las oscilaciones es la existencia de dos frecuencias de oscilación y, además, una de valor doble que la otra. Esto último lo comprobamos mediante la transformada de Fourier, como luego comentaremos. Si realizamos el cálculo teórico del periodo de Bloch que nos proporciona la expresión (4.2) para un nanotubo de 60 celdas y longitud $L=60 a$ sometido a un campo eléctrico de valor $E=813 \mathrm{MV} / \mathrm{m}$, como el representado en la figura 4.9, obtenemos el valor $T_{B}=2,07 \cdot 10^{-14} \mathrm{~s}$. Este valor se corresponde con la frecuencia más elevada en nuestro cálculo numérico. Ahora bien, en el cálculo correspondiente hemos tomado $d$ (el periodo de la red) como la longitud de una celda unidad del nanotubo, por 
a) $(6,6) \quad \mathrm{L}=60 \mathrm{a} \quad \mathrm{E}=813 \mathrm{MV} / \mathrm{m}$

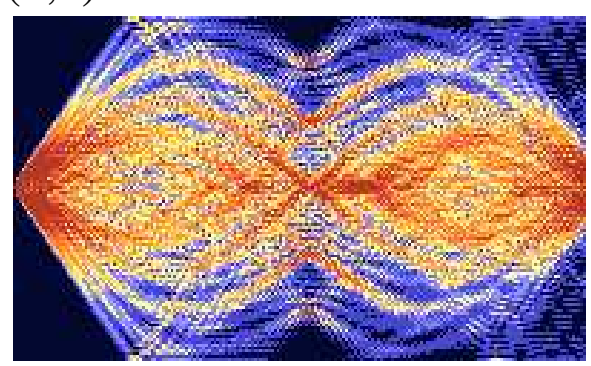

b) $(9,9) \quad \mathrm{L}=60 \mathrm{a} \quad \mathrm{E}=813 \mathrm{MV} / \mathrm{m}$

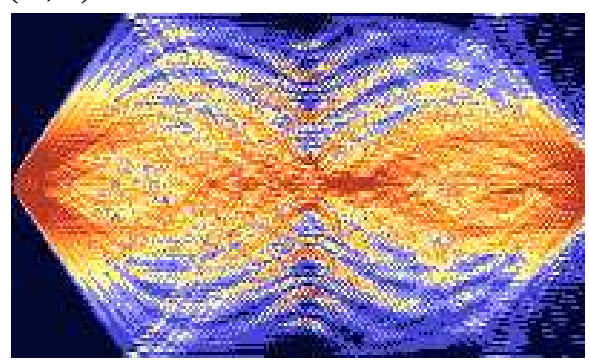

Figura 4.9: Probabilidad de encontrar al electrón en una determinada coordenada $y$ del eje del nanotubo de una onda de Wannier inicial en función del tiempo, para un nanotubo de longitud $L=60 a$. La figura a) muestra los resultados para un nanotubo $(6,6)$, de diámetro igual a $3,3 a$. La figura b) para un nanotubo $(9,9)$ de diámetro $5,0 a$. El valor del campo eléctrico aplicado es en ambos casos $E=813 \mathrm{MV} / \mathrm{m}$. El intervalo de tiempo mostrado va desde $t=0$ hasta $t=4 \cdot 10^{-14} \mathrm{~s}$. Como puede verse, ambas oscilaciones son muy similares.

lo que $d=a$. Es claro que existe una oscilación superpuesta a la anterior de doble periodo que la obtenida a través de nuestro cálculo previo. Esta oscilación puede deberse al hecho de que existe una doble periodicidad en la red. Por un lado, la celda unidad se repite 60 veces a lo largo de todo el nanotubo, a través de esta periodicidad obtenemos el valor $T_{B}=2,07 \cdot 10^{-14} \mathrm{~s}$. Por otro lado, cada celda unidad está compuesta por dos filas de átomos desplazadas lateralmente una respecto a la otra, pero con idéntico número de átomos. Podemos pues decir que las filas de átomos se repiten 120 veces, o lo que es lo mismo, que existe una periodicidad en las filas de átomos igual a $d^{\prime}=a / 2$. Es evidente que tomando este último valor obtenemos un periodo doble que el anterior, es decir: $T_{B}=4,14 \cdot 10^{-14} \mathrm{~s}$., lo que aproximadamente se corresponde con la frecuencia más pequeña que se observa en la figura 4.9. Para clarificar esta situación mostramos 4 celdas de un nanotubo $(4,4)$ desplegado en el plano en la figura 4.10 (la representación no se ha hecho a escala). En esta figura podemos ver las celdas unidad del nanotubo separadas por líneas discontinuas de 
color rojo. La periodicidad de las celdas unidad es $d=a$. Así mismo, se representa la periodicidad de las filas de átomos separadas por líneas de puntos en color azul. La periodicidad de estas líneas es $d^{\prime}=a / 2$, como puede observarse en la figura.

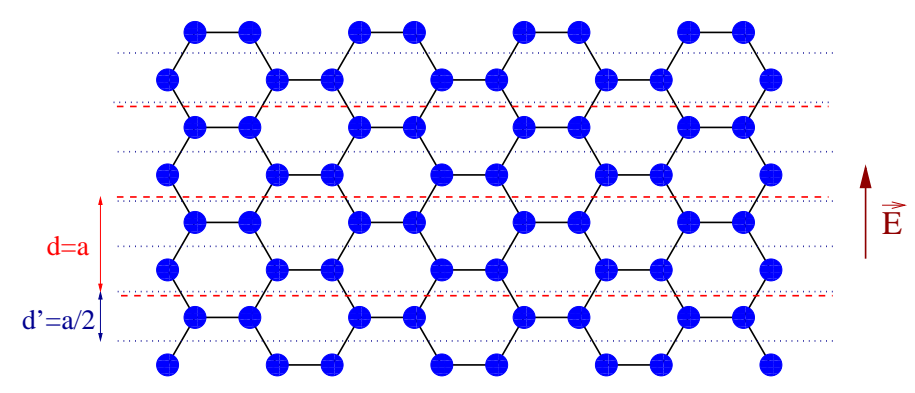

Figura 4.10: Representación esquemática de cuatro celdas unidad de un nanotubo $(4,4)$ desplegado en el plano. El vector en color granate indica la dirección del eje del nanotubo, paralela al campo eléctrico. Se observa una doble periodicidad en la red. Por un lado, las celdas unidad separadas una distancia $d=a$. Por otro lado, las líneas de átomos separadas entre sí una distancia $d^{\prime}=a / 2$. Esta doble periodicidad provoca que la oscilación de la onda posea dos frecuencias, una doble que la otra (La figura no es a escala).

Así pues, el hecho de que las gráficas obtenidas muestren una doble oscilación se debe a que existe también una doble periodicidad en la red, puesto que cada celda unidad contiene exactamente dos filas de átomos. Para corroborar esta afirmación vamos a realizar el cálculo de la probabilidad en una cadena lineal unidimensional de 120 átomos, en la que intercalamos dos energías de sitio diferentes de forma alternativa. Los resultados obtenidos se muestran en la figura 4.11, donde se representa la probabilidad de encontrar al electrón en una determinada coordenada $y$ de una cadena lineal de 120 átomos en función del tiempo, suponiendo la cadena compuesta por 60 celdas unidad de 2 átomos cada una.

Como vemos en la figura 4.11, la forma de la oscilación es similar a las obtenidas en los nanotubos de carbono utilizados en nuestros cálculos. Existe también doble frecuencia, que no puede deberse sino al hecho de que, a pesar de ser una cadena 


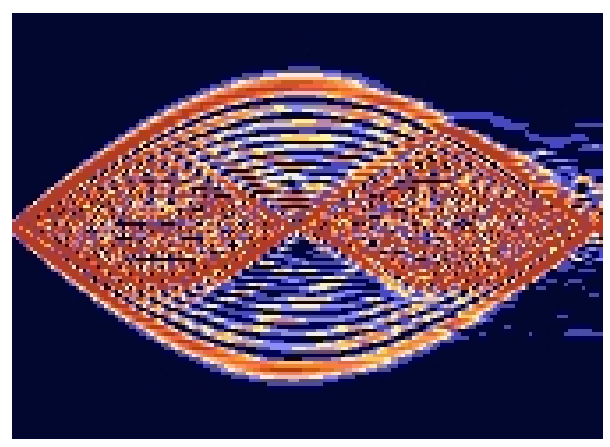

Figura 4.11: Probabilidad de encontrar al electrón en una determinada coordenada $y$ de una cadena lineal de átomos en función del tiempo. La cadena se compone de 120 átomos agrupados en celdas unidad de dos átomos. Se observa una doble periodicidad en la oscilación. Se ha utilizado un campo eléctrico igual a $2033 \mathrm{MV} / \mathrm{m}$.

lineal de 120 átomos, los valores de la energía de sitio en dicha cadena se han ido alternando provocando que la celda unidad de dicha cadena esté compuesta por dos átomos, que se repiten 60 veces. La estructura, por tanto, se puede comparar a un nanotubo tipo armchair con 60 celdas y 120 líneas de átomos, lo cual confirma nuestra hipótesis acerca de la causa de la doble oscilación en los nanotubos de tipo armchair.

\subsubsection{Difusión transversal al campo eléctrico}

En los apartados anteriores hemos mostrado la evolución temporal de la probabilidad de encontrar al electrón en una determinada coordenada $y$ del eje del nanotubo, sumando las probabilidades correspondientes a los átomos con idéntica coordenada $y$. No disponemos de información acerca de la evolución temporal de la función de onda a lo ancho del mismo, lo cual es el objetivo del presente apartado.

El hecho de que nuestro sistema tenga átomos dispuestos transversalmente al campo eléctrico, provoca que la onda se difunda tanto a lo largo como a lo ancho 
del nanotubo. En la difusión horizontal a lo ancho del nanotubo no se producen oscilaciones, ya que no hay campo eléctrico horizontal. En la propagación vertical, la aplicación del campo eléctrico impide que este proceso de difusión de la onda a lo largo del nanotubo se produzca indefinidamente, provocando las reflexiones de Bragg que generan el fenómeno de la localización dinámica. Esto hace que la onda regrese a su posición original, aunque extendida a lo ancho, transcurrido un cierto tiempo.

Para observar gráficamente este fenómeno representamos la probabilidad de encontrar al electrón en una determinada coordenada $y$ del eje del nanotubo en función de la probabilidad de encontrar al electrón en una determinada coordenada $x$. Haremos la representación por medio de una secuencia temporal de imágenes obtenida para un nanotubo $(6,6)$ de longitud $L=60 a$ bajo la influencia de un campo eléctrico $E=2033 \mathrm{MV} / \mathrm{m}$. Dicha secuencia se muestra a lo largo de las dos páginas siguientes (figuras 4.12 y 4.13), a intervalos regulares de tiempo que van desde $t=0$ hasta $t \approx 8,5 \cdot 10^{-15} \mathrm{~s}$. Si establecemos un paralelismo con la representación de la figura $4.4 \mathrm{c}$ ), estaríamos representando una oscilación similar (con diferente valor de $E$ ) pero aquí podemos visualizar lo que sucede en cada instante en la sección transversal del nanotubo.

Como vemos en la secuencia de imágenes, la onda inicialmente localizada en un único átomo se va extendiendo a lo largo y a lo ancho del nanotubo. Transcurrido un cierto tiempo, la onda regresa a la posición y inicial, pero permanece extendida a lo ancho del nanotubo, ya que no existe campo eléctrico en esta dirección. La secuencia muestra poco más de una oscilación considerando el periodo calculado anteriormente. En la figura 4.12, se indica con un asterisco la imagen que se corresponde con un tiempo $t \approx 4,2 \cdot 10^{-15} \mathrm{~s}$, es decir, se correspondería con la región intermedia en la figura $4.4 \mathrm{c}$ ). En la 4.13 , se indica con un asterisco la probabilidad para tiempo $t \approx 8,3 \cdot 10^{-15} \mathrm{~s}$, es decir, lo que sería la parte final en la figura $4.4 \mathrm{c}$ ). 

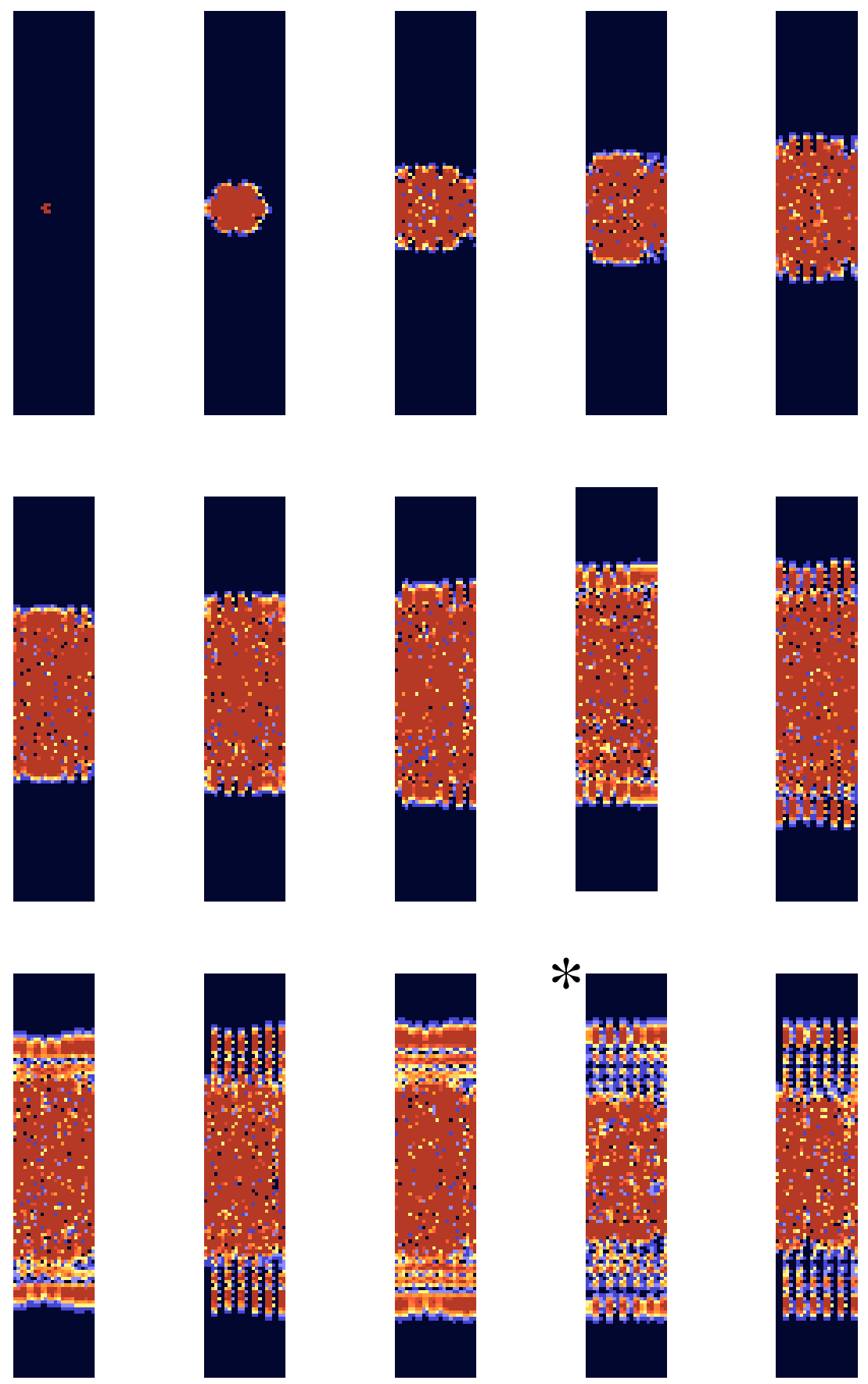

Figura 4.12: Probabilidad de encontrar al electrón en una determinada coordenada $y$ del eje del nanotubo en función de la probabilidad a lo largo del eje $x$, en una secuencia temporal de imágenes a intervalos regulares de tiempo. La figura señalada con asterisco se corresponde con un tiempo $t \approx 4,2 \cdot 10^{-15} \mathrm{~s}$. 

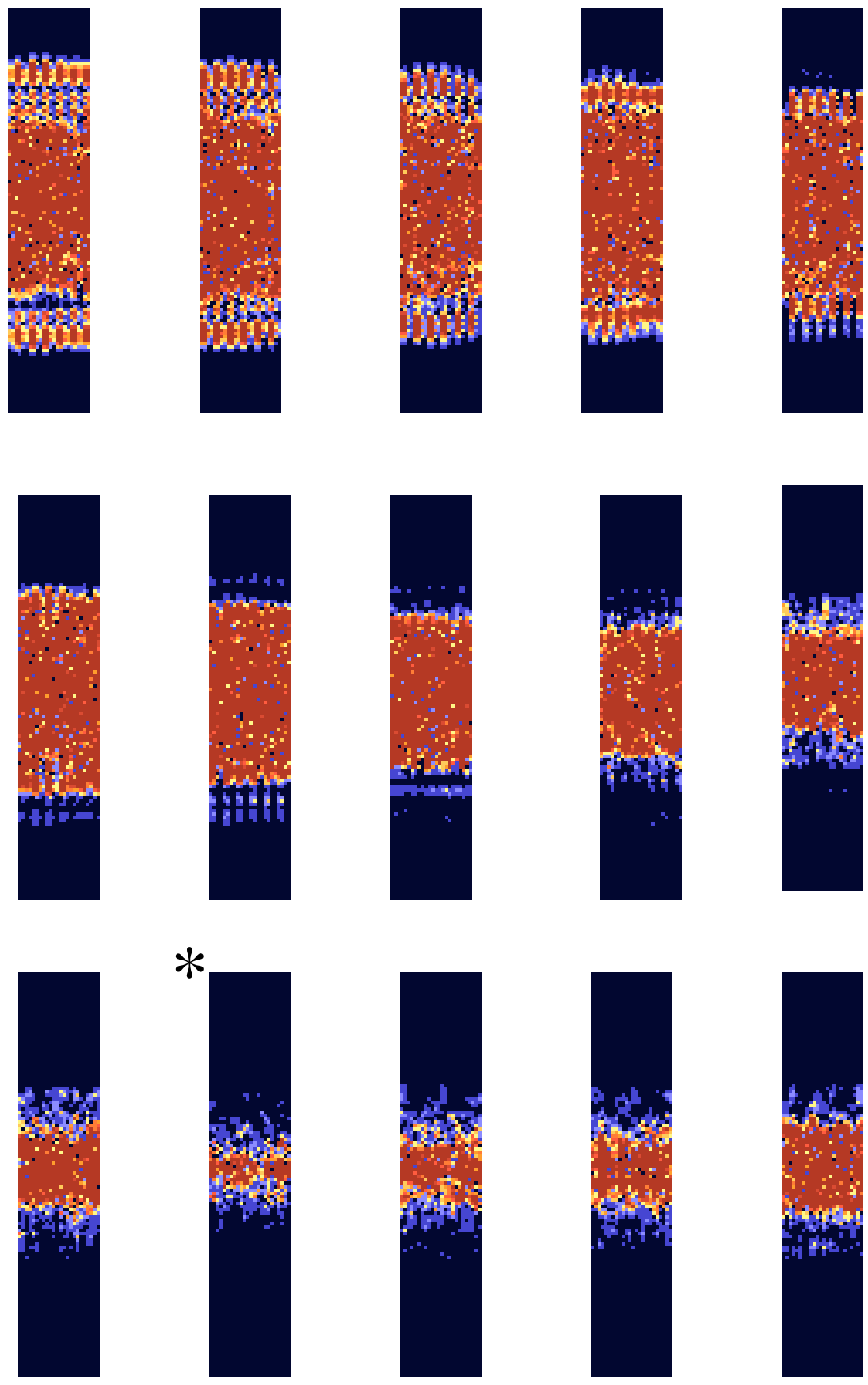

Figura 4.13: Continuación de la secuencia temporal mostrada en la página anterior. La onda señalada con asterisco se corresponde con un tiempo $t \approx 8,3 \cdot 10^{-15} \mathrm{~s}$. 

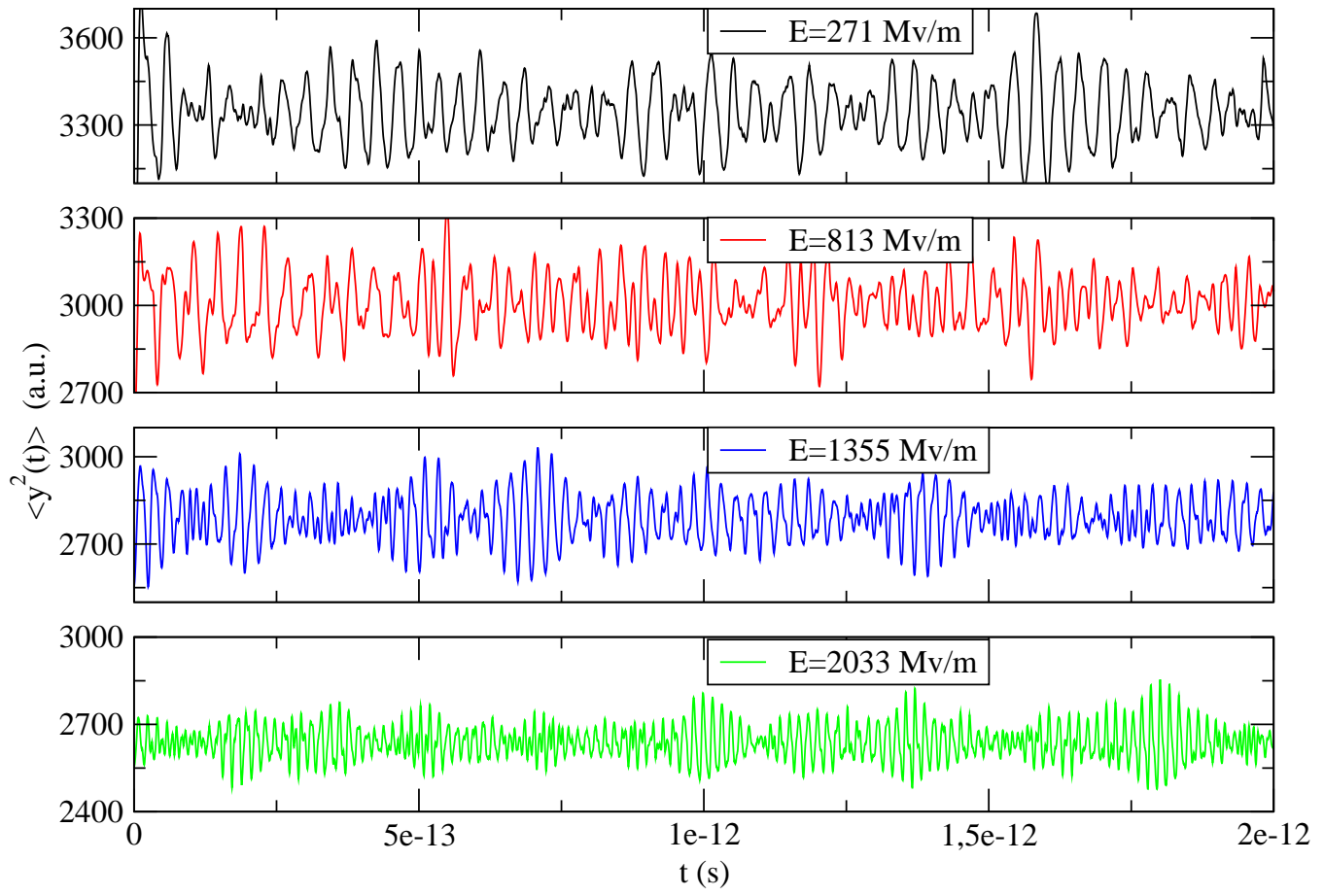

Figura 4.14: Desplazamiento cuadrático medio de una función de onda inicial de Wannier en un nanotubo $(6,6)$ de longitud $L=60 a$ para diferentes valores del campo eléctrico. Desde la parte superior hasta la inferior $E=271$ $\mathrm{MV} / \mathrm{m}, E=813 \mathrm{MV} / \mathrm{m}, E=1355 \mathrm{MV} / \mathrm{m}$ y $E=2033 \mathrm{MV} / \mathrm{m}$, respectivamente. 


\subsubsection{Desplazamiento cuadrático medio}

El desplazamiento cuadrático medio de la función de onda lo obtendremos a partir de la expresión (4.18):

$$
\left\langle y^{2}(t)\right\rangle=\sum_{i}\left|\Psi^{i}(E, t)\right|^{2}\left|y_{i}\right|^{2},
$$

donde recordemos que $\Psi^{i}(E, t)$ es la amplitud de probabilidad de encontrar al electrón en el átomo $i$, y donde la suma se extiende evidentemente sobre todos los átomos del nanotubo, siendo $y_{i}$ la coordenada $y$ sobre el eje del nanotubo del átomo correspondiente. Esta función debe mostrar un comportamiento oscilatorio en función del tiempo, al igual que lo hace la probabilidad tal y como predice la teoría de Bloch. Como ya hemos mencionado anteriormente, el cálculo del desplazamiento cuadrático medio nos permitirá obtener la frecuencia de la oscilación pruducida mediante el uso de la transformada de Fourier de dicha función. Comenzaremos por obtener los resultados para las funciones de onda iniciales completamente localizadas en un átomo (ondas de Wannier).

En la figura 4.14 se muestra el desplazamiento cuadrático medio de una función de onda inicial de Wannier en un nanotubo $(6,6)$ de longitud $L=60 a$, para diferentes valores del campo eléctrico aplicado. Los valores del campo eléctrico aplicado son, desde la parte superior hasta la parte inferior de dicha figura, $E=271 \mathrm{MV} / \mathrm{m}$, $E=813 \mathrm{MV} / \mathrm{m}, E=1355 \mathrm{MV} / \mathrm{m}$ y $E=2033 \mathrm{MV} / \mathrm{m}$. Se aprecia claramente en la figura cómo a medida que va aumentando el campo eléctrico, aumenta también la frecuencia de la oscilación. Por contra, la amplitud de la oscilación disminuye con el aumento del campo eléctrico. Este comportamiento se corresponde con el que predicen las ecuaciones (4.2) y (4.3). 

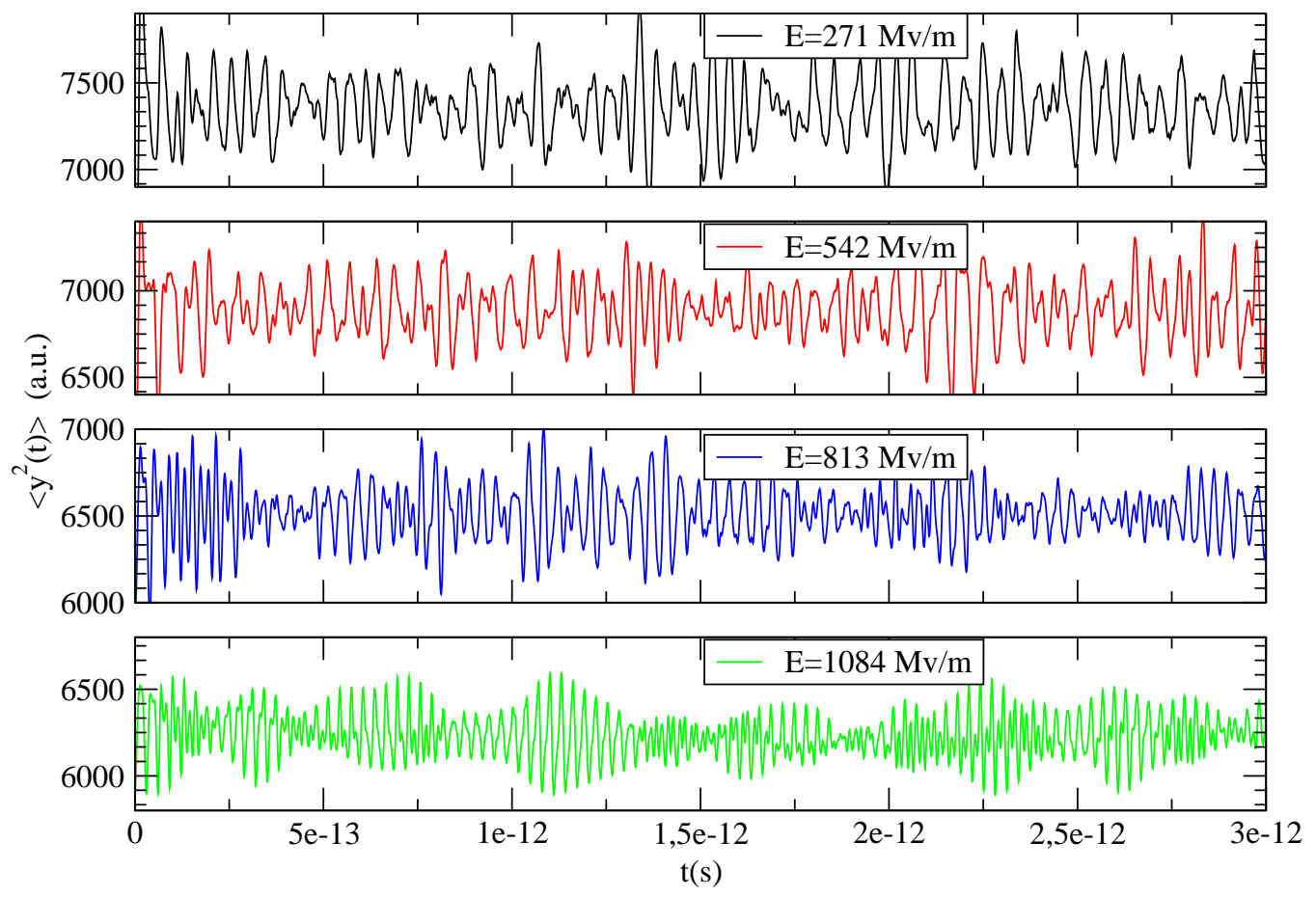

Figura 4.15: Desplazamiento cuadrático medio de una función de onda inicial de Wannier en un nanotubo $(6,6)$ de longitud $L=90 a$. Desde la parte superior hasta la inferior de la figura los valores del campo eléctrico son $E=271 \mathrm{MV} / \mathrm{m}, E=542 \mathrm{MV} / \mathrm{m}, E=813 \mathrm{MV} / \mathrm{m}$ y $E=1084 \mathrm{MV} / \mathrm{m}$.

En la figura 4.15 se muestra el desplazamiento cuadrático medio de una función de onda inicial de Wannier para un nanotubo $(6,6)$ de longitud $L=90 a$, para diferentes valores del campo eléctrico. Desde la parte superior hasta la inferior de dicha figura, los valores del campo eléctrico aplicado son $E=271 \mathrm{MV} / \mathrm{m}, E=$ $542 \mathrm{MV} / \mathrm{m}, E=813 \mathrm{MV} / \mathrm{m}$ y $E=1084 \mathrm{MV} / \mathrm{m}$. Al igual que para el nanotubo de longitud $L=60 a$, se aprecia que a medida que aumenta el campo eléctrico, aumenta la frecuencia de la oscilación y disminuye la amplitud de la misma. Las mismas características presentan las funciones representadas en la figura 4.16 para 
un nanotubo de longitud $L=180 a$, para idénticos valores del campo eléctrico que en la figura 4.15. Como se puede observar en las tres figuras anteriores (4.14, $4.15 \mathrm{y}$ 4.16), el desplazamiento cuadrático medio presenta un comportamiento oscilatorio y periódico frente al tiempo, por lo que el siguiente paso será obtener la frecuencia (o frecuencias) de dichas oscilaciones.

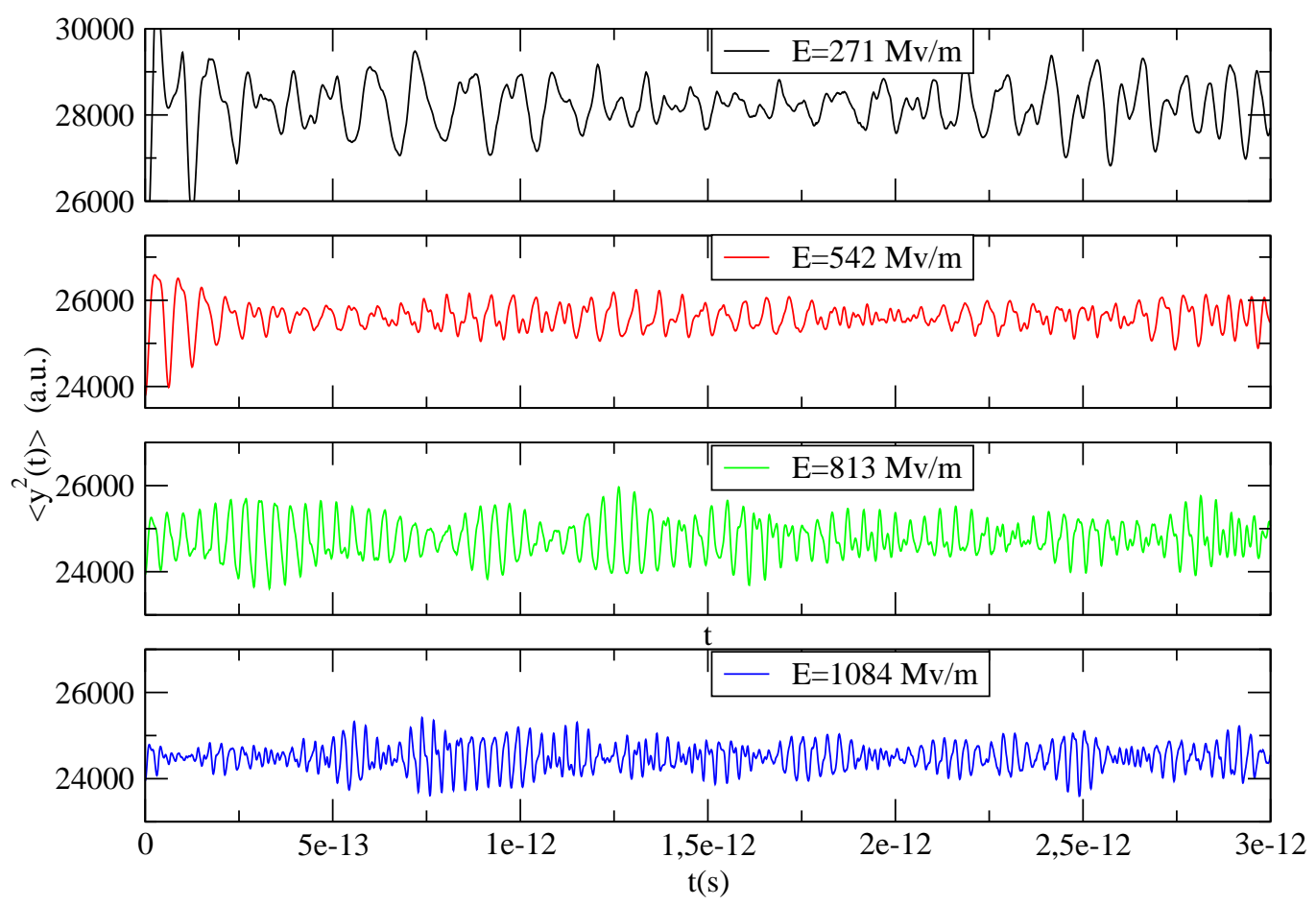

Figura 4.16: Desplazamiento cuadrático medio de una función de onda inicial de Wannier en un nanotubo $(6,6)$ de longitud $L=180 a$. Desde la parte superior hasta la inferior de la figura los valores del campo eléctrico son $E=271 \mathrm{MV} / \mathrm{m}, E=542 \mathrm{MV} / \mathrm{m}, E=813 \mathrm{MV} / \mathrm{m}$ y $E=1084 \mathrm{MV} / \mathrm{m}$, respectivamente. 


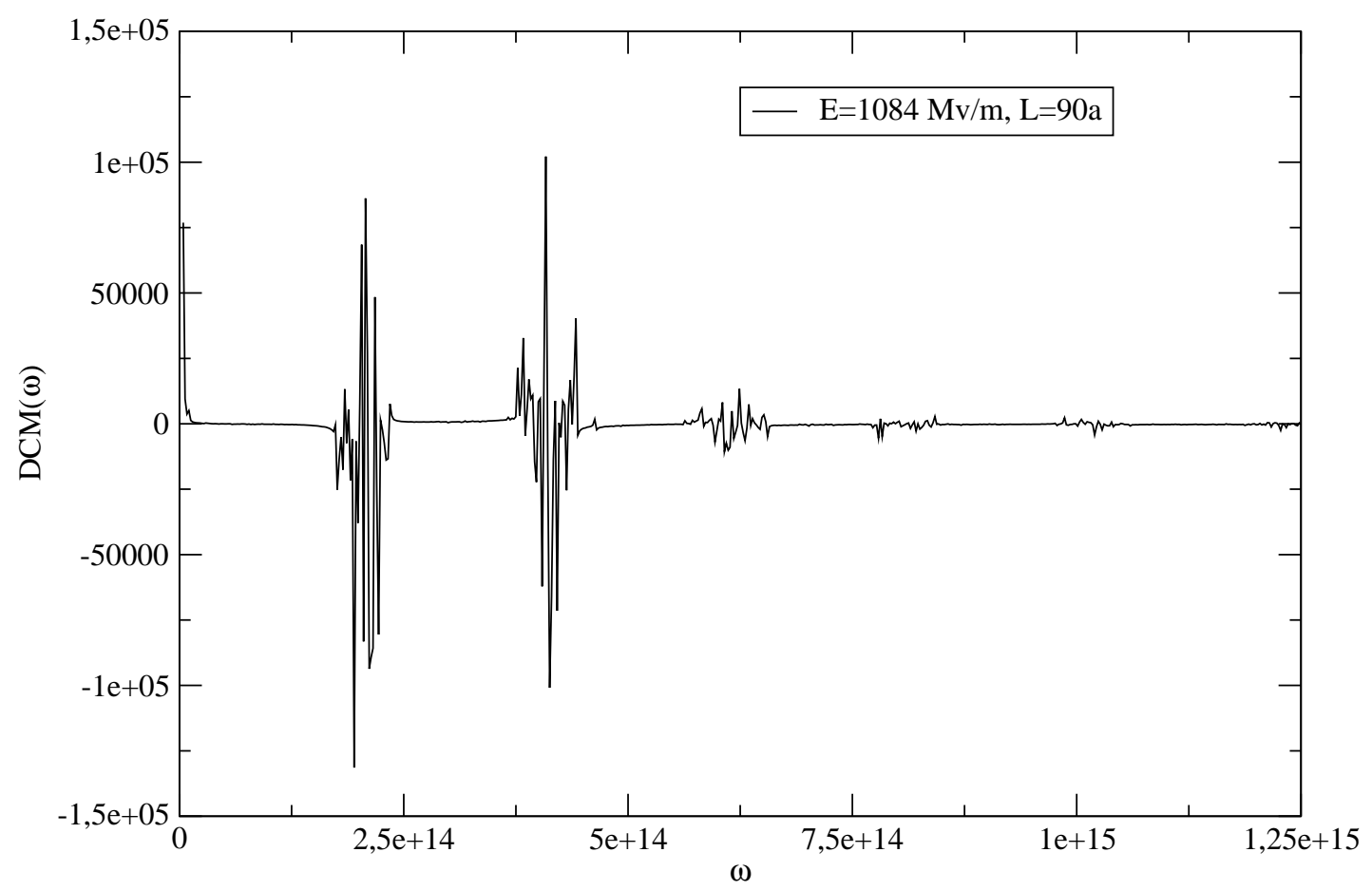

Figura 4.17: Transformada de Fourier del desplazamiento cuadrático medio en función de la frecuencia angular. Los resultados que se muestran se corresponden con los cálculos realizados para un nanotubo $(6,6)$ de longitud $L=90 a$ y un campo eléctrico igual a $E=1084 \mathrm{MV} / \mathrm{m}$. La figura pone de manifiesto la existencia de dos frecuencias de oscilación principales, como se discute en el texto.

La frecuencia de la oscilación del desplazamiento cuadrático medio se puede obtener, como ya se ha mencionado anteriormente, a través del cálculo de la transformada de Fourier. En la figura 4.17 se muestra, a modo de ejemplo, el valor de dicha transformada en función de la frecuencia angular $\omega$, para un nanotubo $(6,6)$ de longitud $L=90 a$ y un campo eléctrico igual a $E=1084 \mathrm{MV} / \mathrm{m}$. Como vemos en dicha figura, la oscilación presenta dos frecuencias principales, como ya adelantamos en apartados anteriores. Podemos volver a ver la gráfica de la probabilidad frente al 
tiempo para este caso particular (figura $4.5 \mathrm{~d}$ )) donde, como dijimos, se observan dos oscilaciones de frecuencia una doble que la otra. Comparando las frecuencias que podemos extrapolar de esas figuras, vemos que se corresponden fielmente con los picos de la transformada de Fourier. Tomando únicamente la frecuencia más alta de las dos podemos chequear la validez de la expresión teórica (4.2) obtenida para cadenas unidimensionales de átomos. En la gráfica 4.18 mostramos la frecuencia de la oscilación del desplazamiento cuadrático medio frente al campo eléctrico aplicado. La recta se corresponde con el valor teórico predicho por la ecuación (4.2). Los símbolos representan los valores obtenidos mediante nuestro cálculo numérico en nanotubos de carbono. Se muestran los resultados obtenidos para nanotubos de diferente longitud. Aunque los valores teóricos de la frecuencia no dependen de la longitud, los efectos de borde pueden provocar ligeras diferencias en el cálculo de los mismos. Así, se presentan los resultados mediante diferentes símbolos. Como puede observarse en las figuras, los resultados numéricos con nanotubos de carbono reproducen fielmente lo predicho por la teoría para cadenas unidimensionales de átomos.

\subsection{Oscilaciones de Bloch en nanotubos puros. On- das extendidas}

$\mathrm{Si}$ en lugar de tomar como estado inicial ondas localizadas en un único átomo tomamos las autofunciones del Hamiltoniano del sistema sin campo eléctrico, tendremos ondas extendidas a lo largo de todo el nanotubo. Podremos estudiar entonces su evolución a lo largo del tiempo cuando aplicamos un campo eléctrico. Para ello, utilizamos nuevamente la expresión (4.16). En este caso la teoría predice una evolución dinámica de todo el paquete de ondas, en la que el centro del paquete sí oscila, al contrario de lo que sucede con las ondas inicialmente localizadas. 


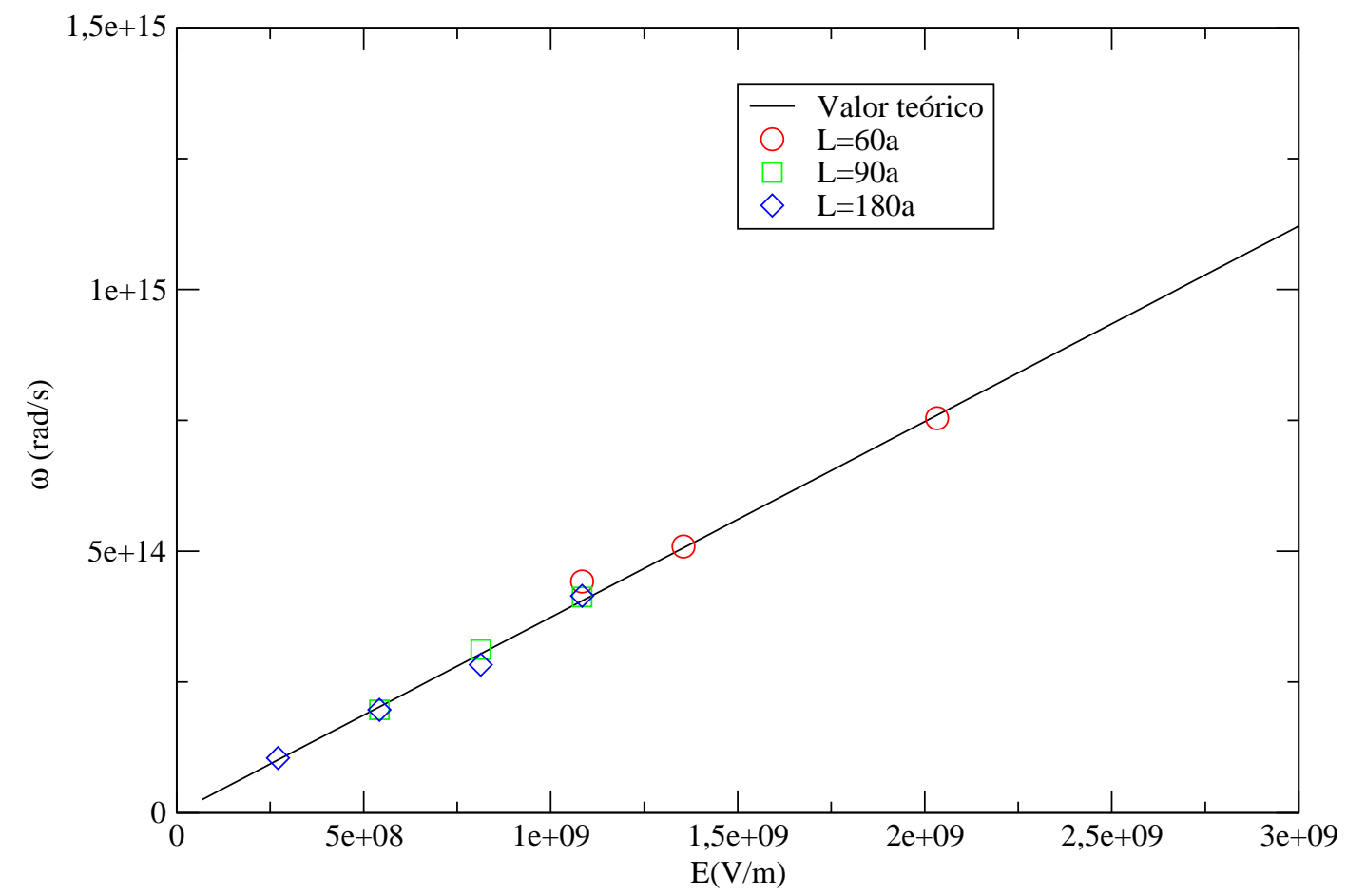

Figura 4.18: Frecuencia angular de la oscilación del desplazamiento cuadrático medio frente al campo eléctrico aplicado. La línea recta representa el valor teórico predicho por la ecuación (4.2). Los símbolos representan los valores obtenidos mediante el cálculo numérico, obtenido para diferentes longitudes del sistema. La correspondencia es clara. 


\subsubsection{Funciones de onda. Probabilidad de ocupación}

Comenzamos por mostrar en este apartado la evolución temporal de la probabilidad de encontrar al electrón en una determinada coordenada $y$ del eje del nanotubo. El cálculo lo hacemos para un CNT $(6,6)$ de diferentes longitudes, y sometido a diferentes valores del campo eléctrico.

Los resultados obtenidos en este caso se muestran en las figuras 4.19, 4.20 y 4.21 para nanotubos $(6,6)$ de longitudes $L=60 a, L=90 a$ y $L=180 a$, respectivamente. Las figuras representadas permiten apreciar visualmente la existencia de oscilaciones de la función de onda a lo largo del tiempo, que se corresponden con oscilaciones de Bloch de frecuencia dada por la ecuación (4.2), como veremos en el siguiente apartado. Al contrario de lo que ocurría con las ondas inicialmente localizadas estudiadas anteriormente, el centro del paquete de ondas oscila al aplicar un campo eléctrico constante. Para cada longitud se ha representado la probabilidad al aplicar diferentes valores del campo eléctrico. Como referencia se muestra en el apartado a) de las tres figuras la evolución del paquete de ondas en ausencia de campo eléctrico y se observa que esta onda no cambia con el tiempo, como era de esperar. En la subfigura b) de las tres gráficas se aplica un campo eléctrico pequeño donde las ondas comienzan a oscilar. Sin embargo, debido a que la amplitud y el periodo son grandes, se reflejan en los extremos y no se llega a apreciar ninguna oscilación sinusoidal. Por último se muestra en el apartado c) de las tres gráficas, una figura con un campo eléctrico más intenso donde ya se aprecia la oscilación del paquete de ondas. Comparando esta subfigura en las gráficas 4.19, 4.20 y 4.21, vemos cómo al aumentar el tamaño del nanotubo las oscilaciones se muestran más claras. Esto es debido a que al ir aumentando la longitud del nanotubo los efectos de tamaño finito son menos importantes. 

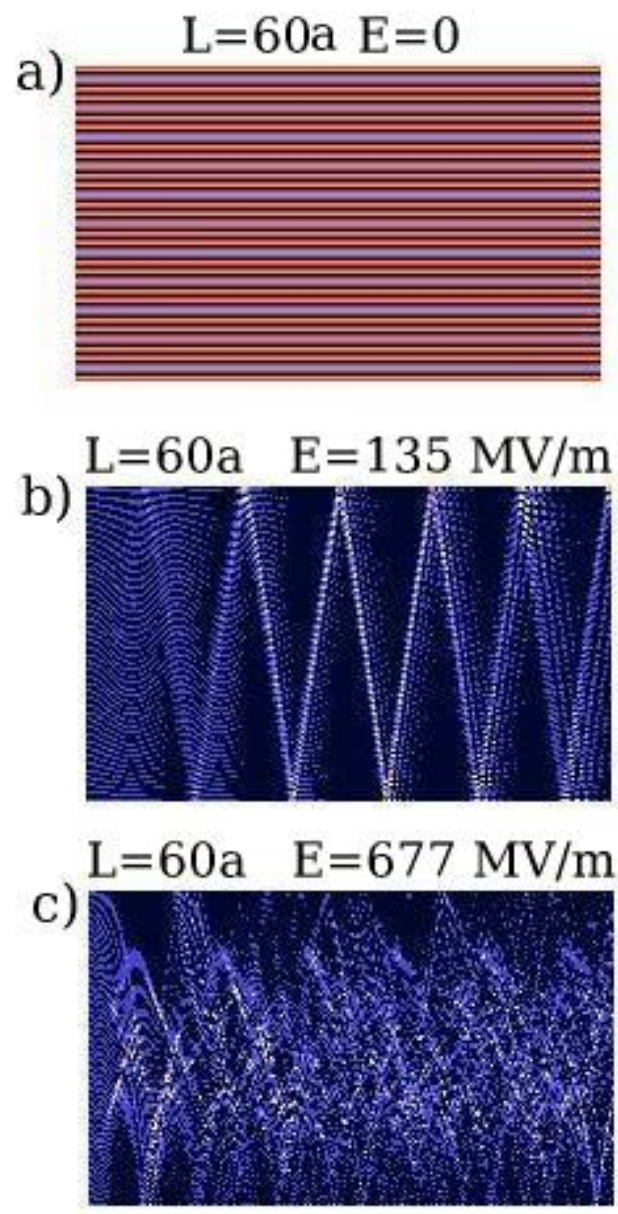

Figura 4.19: Probabilidad de encontrar al electrón en una determinada coordenada $y$ del eje del nanotubo frente al tiempo para un CNT de longitud $L=60 a$. La figura a) muestra la autofunción de onda sin campo eléctrico, que no evoluciona temporalmente. Las figuras b) y c) se corresponden con los valores del campo eléctrico $E=135 \mathrm{MV} / \mathrm{m}$ y $677 \mathrm{MV} / \mathrm{m}$, respectivamente. En la figura b) vemos cómo el electrón se refleja en los bordes, por lo que no se aprecian oscilaciones de Bloch. En la figura c), sin embargo, éstas sí se ponen de manifiesto. 

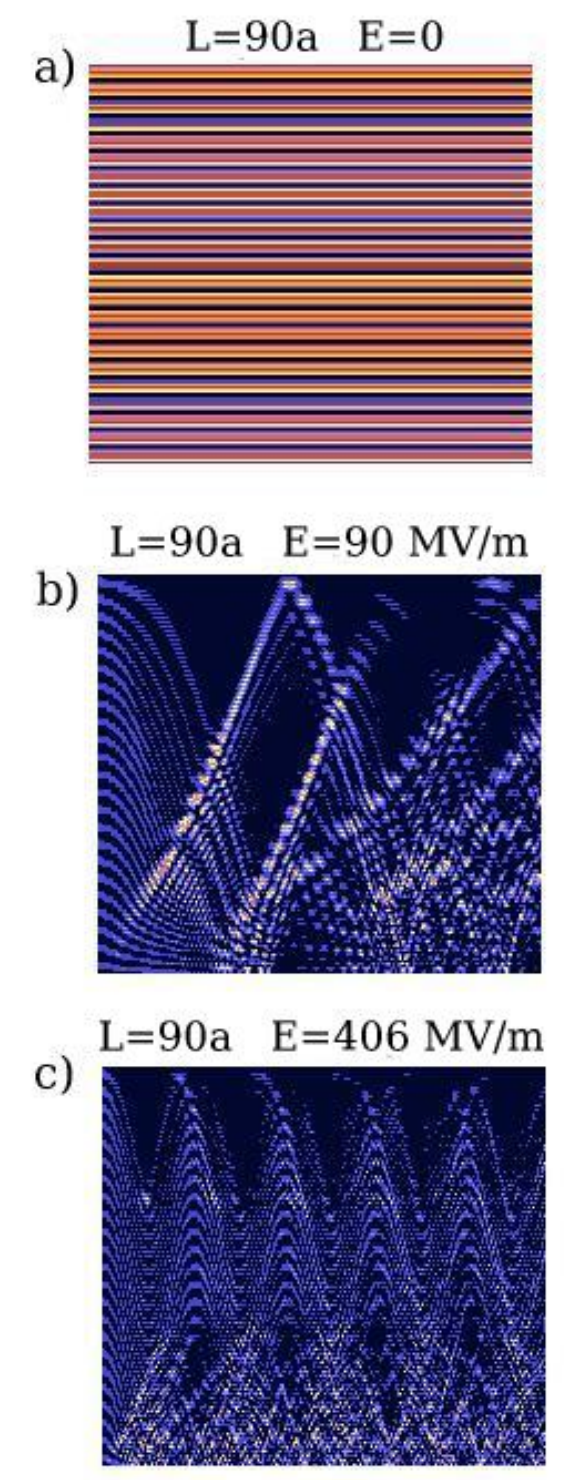

Figura 4.20: Probabilidad de encontrar al electrón en una determinada coordenada $y$ del eje del nanotubo frente al tiempo para un CNT de longitud $L=90 a$. La figura a) muestra la autofunción de onda sin campo eléctrico. Las figuras b) y c) se corresponden con los valores del campo eléctrico $E=90$ $\mathrm{MV} / \mathrm{m}$ y $406 \mathrm{MV} / \mathrm{m}$, respectivamente. En la figura b) vemos cómo el electrón se refleja en los bordes, por lo que no se aprecian oscilaciones de Bloch. En la figura c), sin embargo, éstas sí se ponen de manifiesto. 

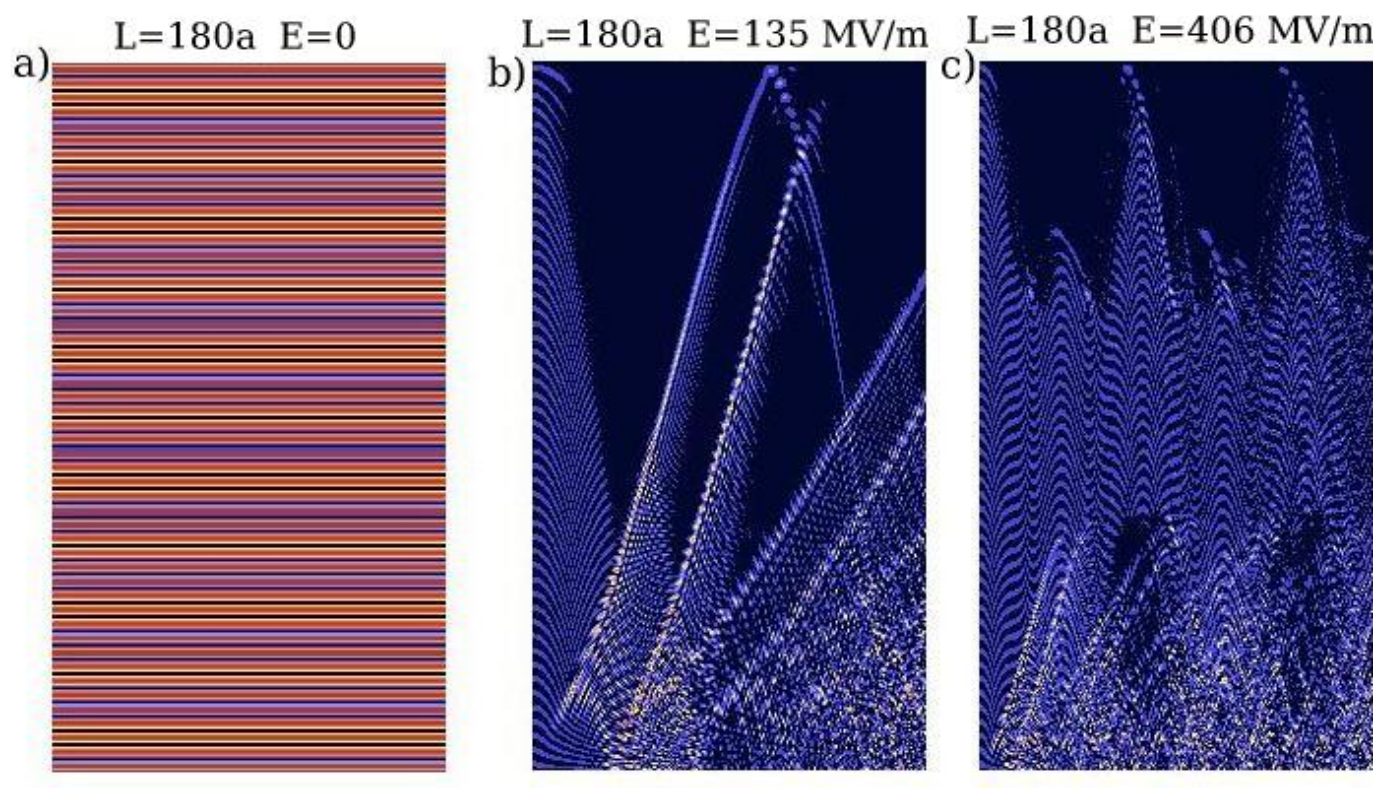

Figura 4.21: Probabilidad de encontrar al electrón en una determinada coordenada $y$ del eje del nanotubo frente al tiempo para un CNT de longitud $L=180 a$. La figura a) muestra la autofunción de onda sin campo eléctrico. Las figuras b) y c) se corresponden con los valores del campo eléctrico $E=135 \mathrm{MV} / \mathrm{m}$ y $406 \mathrm{MV} / \mathrm{m}$, respectivamente. En la figura b) vemos cómo el electrón se refleja en los bordes, por lo que no se aprecian oscilaciones de Bloch. En la figura c), sin embargo, éstas sí se ponen de manifiesto. 


\subsubsection{Desplazamiento cuadrático medio}

Como ya hicimos en el apartado 4.3.4 para ondas localizadas, obtendremos aquí el desplazamiento cuadrático medio en función del tiempo para nanotubos $(6,6)$ de diferentes longitudes y con distintos valores del campo eléctrico. El posterior análisis de Fourier de estas funciones nos permitirá obtener la frecuencia de oscilación de las mismas. Podremos, entonces, comparar los resultados de nuestro cálculo para ondas inicialmente extendidas en nanotubos de carbono con la expresión teórica (4.2).

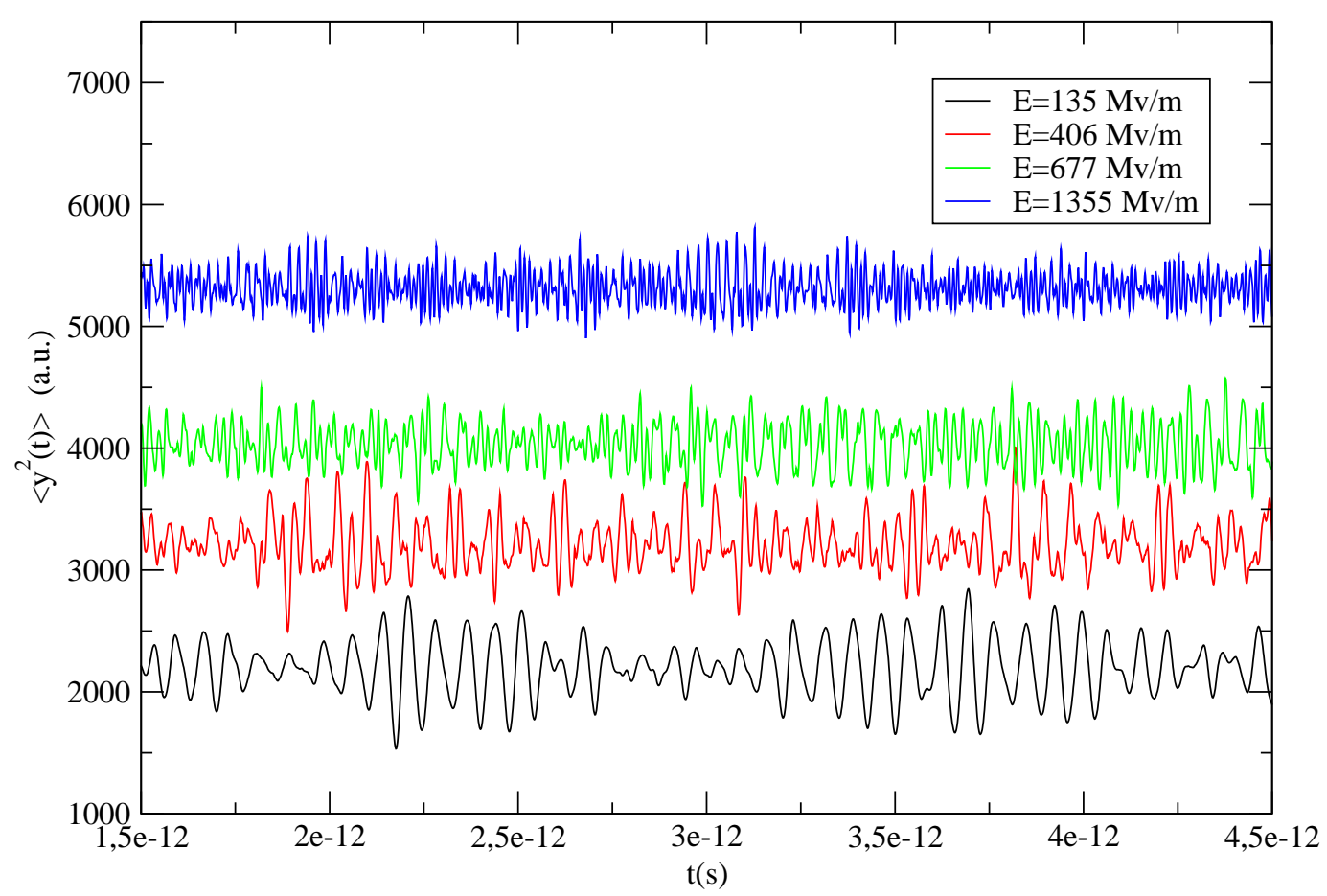

Figura 4.22: Desplazamiento cuadrático medio en función del tiempo para la transmisión de una función de onda en un nanotubo tipo $(6,6)$ de longitud $\mathrm{L}=60 \mathrm{a}$. Se muestra dicha función para diferentes valores del campo eléctrico. Las curvas se representan en unidades arbitrarias, ya que están desplazadas verticalmente con el fin de visualizarlas de forma más clara. 
En la figura 4.22 se muestran los resultados obtenidos para un nanotubo de longitud $L=60 a$. Se ha representado en dicha figura el desplazamiento cuadrático medio en función del tiempo cuando el nanotubo está sometido a diferentes valores del campo eléctrico. Como se aprecia claramente en la figura, al aumentar el campo eléctrico aplicado aumenta la frecuencia de las oscilaciones, a la vez que disminuye su amplitud. Este comportamiento se corresponde con el que predicen las ecuaciones (4.2) y (4.3), como ya hemos dicho anteriormente.

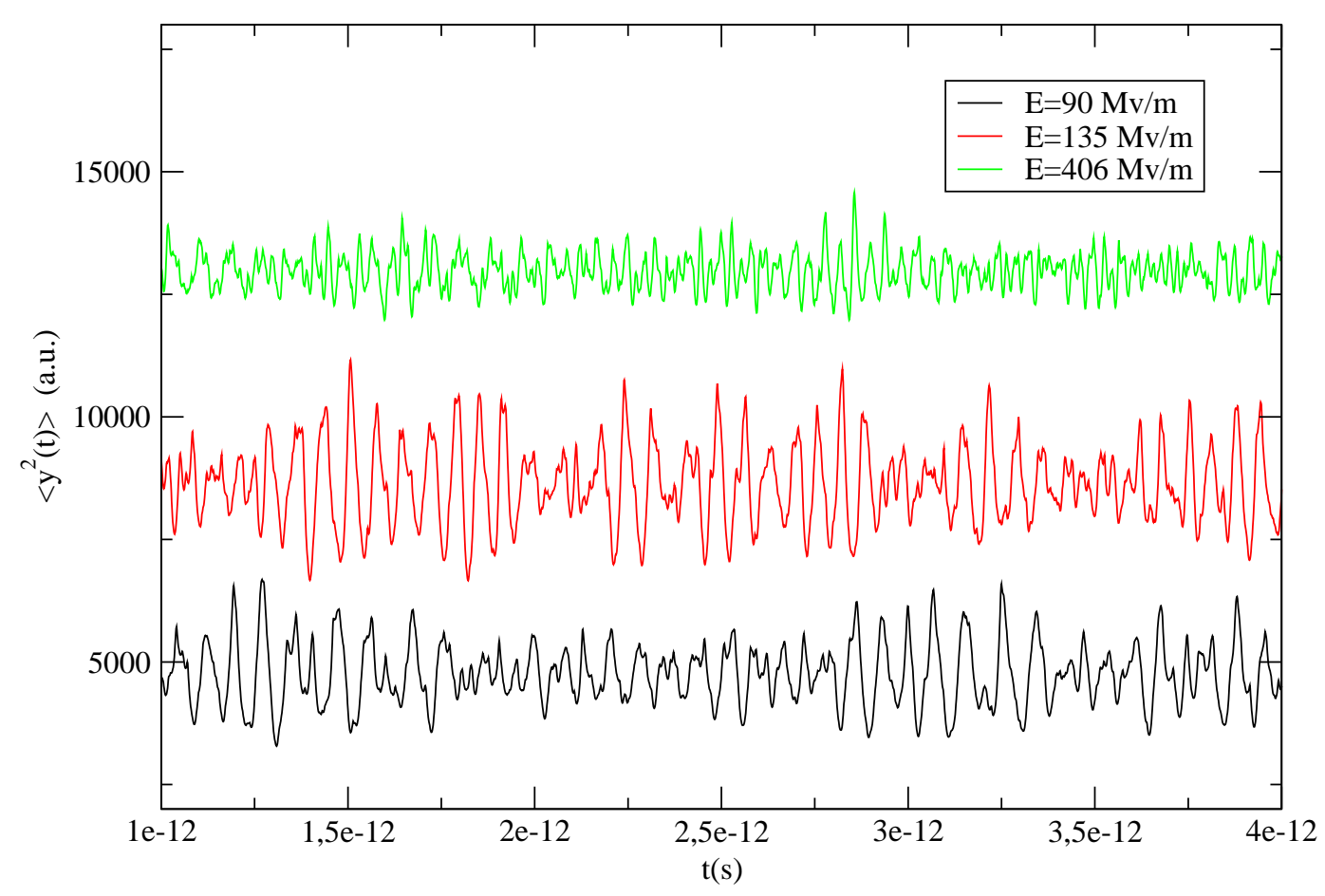

Figura 4.23: Desplazamiento cuadrático medio en función del tiempo para la transmisión de una función de onda en un nanotubo tipo $(6,6)$ de longitud $\mathrm{L}=90$ a. Se muestra dicha función para diferentes valores del campo eléctrico. Las curvas se representan en unidades arbitrarias, ya que están desplazadas verticalmente con el fin de visualizarlas de forma más clara. 


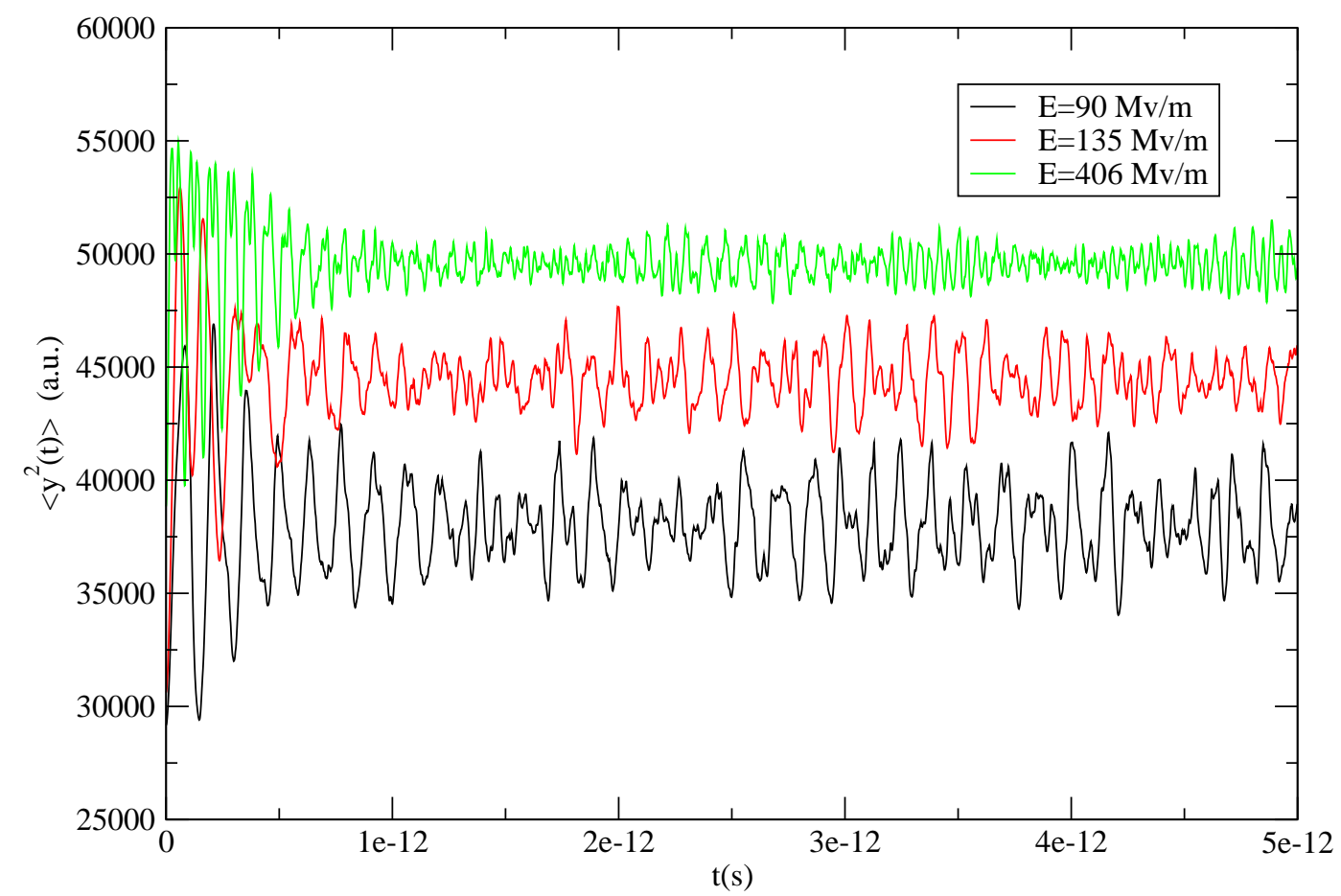

Figura 4.24: Desplazamiento cuadrático medio en función del tiempo para la transmisión de una función de onda en un nanotubo tipo $(6,6)$ de longitud $\mathrm{L}=180$ a. Se muestra dicha función para diferentes valores del campo eléctrico. Las curvas se representan en unidades arbitrarias, ya que están desplazadas verticalmente con el fin de visualizarlas forma más clara. 
En las figuras 4.23 y 4.24 se muestran los resultados obtenidos para diferentes valores del campo eléctrico para nanotubos de longitud $L=90 a$ y $L=180 a$, respectivamente. Ambas representaciones muestran el mismo tipo de dependencia de la frecuencia y la amplitud de la onda con el potencial que en la figura 4.22. Sin embargo, a medida que la longitud del nanotubo es mayor, los efectos de borde afectan menos a los resultados y podemos obtener la frecuencia de la oscilación de manera más fiable al igual que hicimos para las ondas inicialmente localizadas.

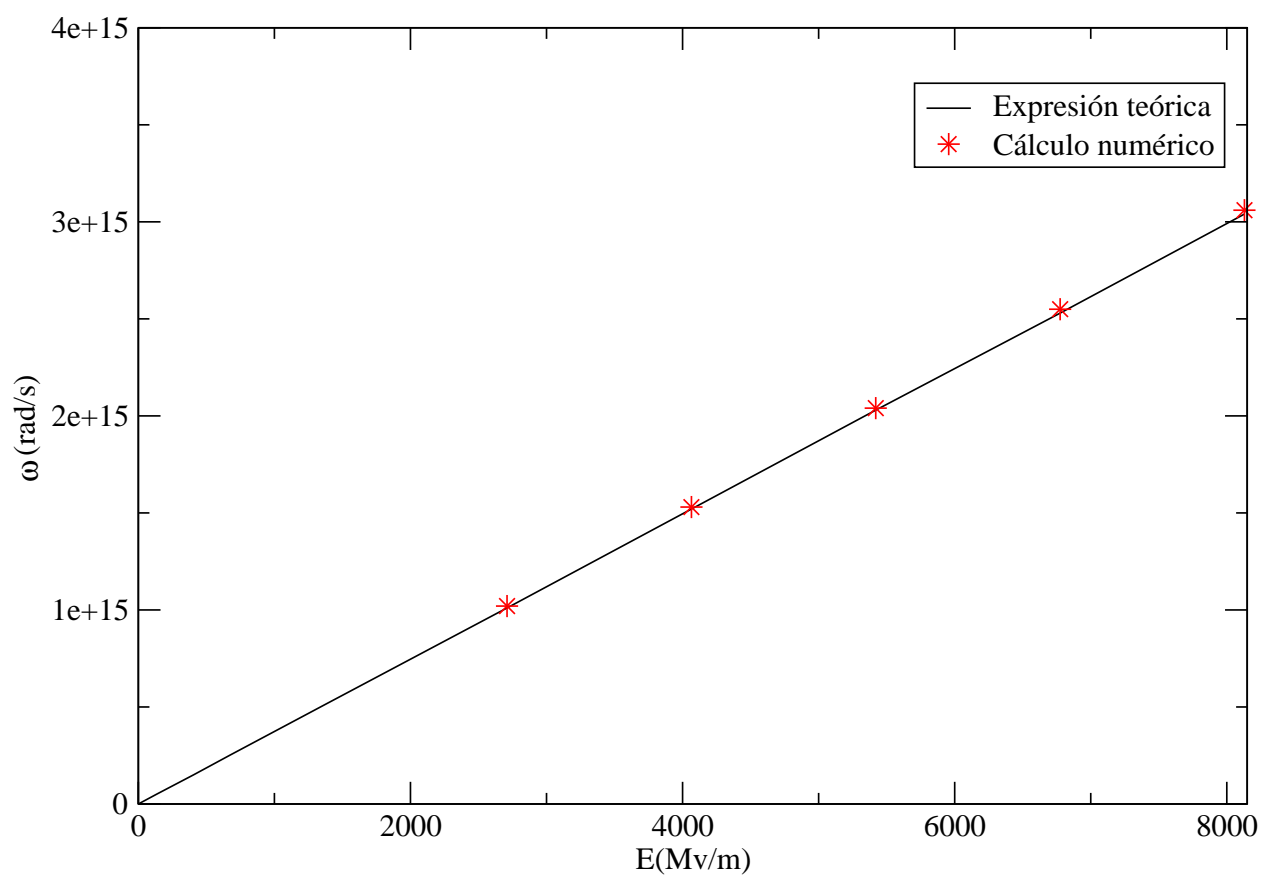

Figura 4.25: Frecuencia de la oscilación del desplazamiento cuadrático medio en función del campo eléctrico. La línea continua se corresponde con el valor teórico predicho por la ecuación (4.2). Los puntos señalados con asterisco son los obtenidos mediante el cálculo numérico desarrollado para un nanotubo $(6,6)$. Existe un grado de correspondencia muy alto entre los valores teóricos y los valores numéricos, como puede verse en la figura. 
Para concluir este apartado, en la figura 4.25 se muestra la frecuencia de la oscilación del desplazamiento cuadrático medio en función de la diferencia de potencial, según la expresión teórica dada por la ecuación (4.2), y según los resultados obtenidos mediante nuestro cálculo. Nuevamente, como en el caso de las ondas iniciales de Wannier, se aprecia una muy buena correspondencia entre la expresión teórica para cadenas unidimensionales de átomos y nuestros cálculos realizados para nanotubos de carbono con ondas inicialmente extendidas.

\subsubsection{Función de participación}

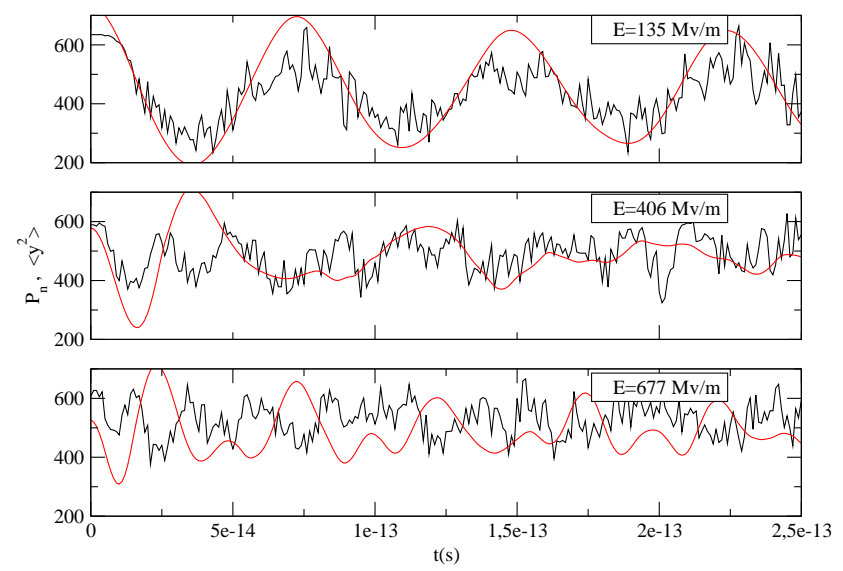

Figura 4.26: Función de participación y desplazamiento cuadrático medio en función del tiempo para la transmisión de una función de onda extendida en un nanotubo tipo $(6,6)$ de longitud $L=60 a$. Se muestran los resultados para diferentes valores del campo eléctrico. Para comparar dichas funciones el desplazamiento cuadrático medio se expresa en unidades arbitrarias.

Hasta el momento, hemos obtenido la frecuencia de las oscilaciones de Bloch a través del cálculo de la transformada de Fourier del desplazamiento cuadrático medio a lo largo del eje del nanotubo. Las frecuencias obtenidas mediante este cálculo nos daban valores bastante exactos cuando los comparábamos con los teóricos. Otra 


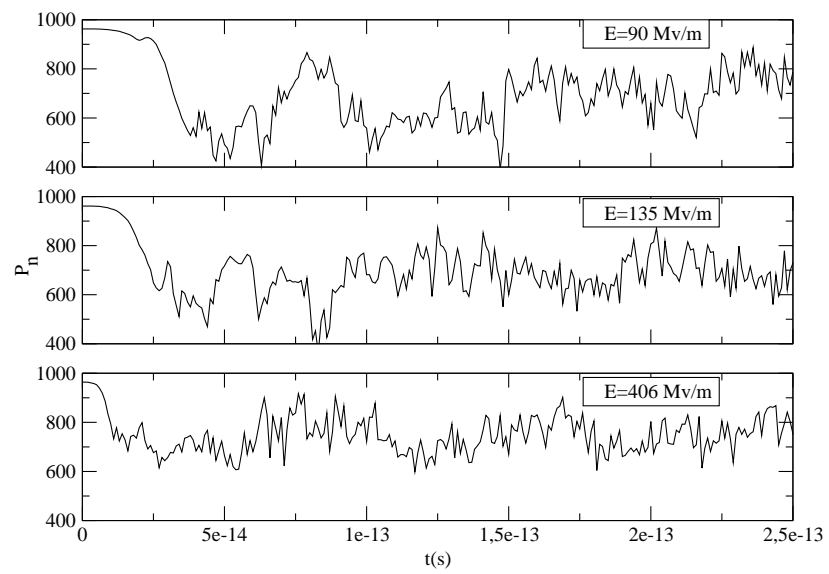

Figura 4.27: Función de participación en función del tiempo para la transmisión de una función de onda extendida en un nanotubo tipo $(6,6)$ de longitud $L=90 a$. Se muestra dicha función para diferentes valores del campo eléctrico.
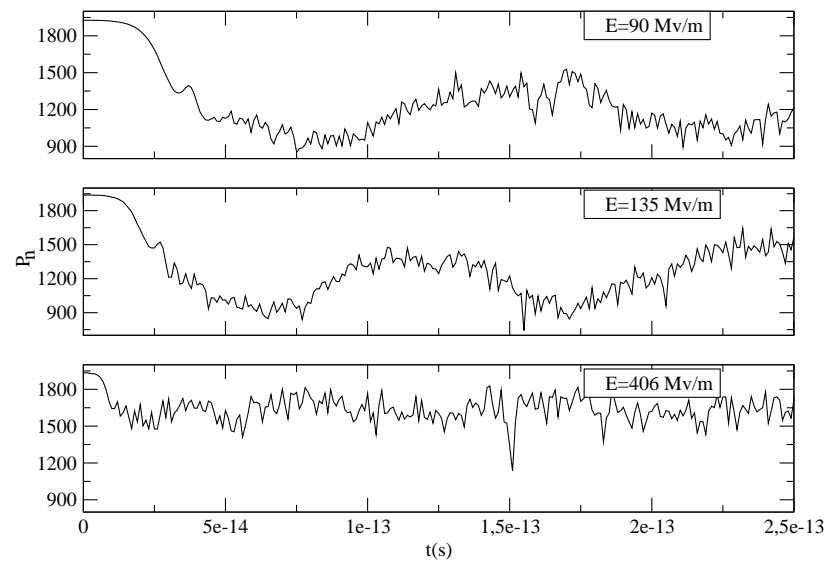

Figura 4.28: Función de participación en función del tiempo para la transmisión de una función de onda extendida en un nanotubo tipo $(6,6)$ de longitud $L=180 a$. Se muestra dicha función para diferentes valores del campo eléctrico. 
magnitud física relacionada con la dispersión del paquete de ondas en el espacio es la función de participación (apartado 2.1.1), una magnitud que conviene también estudiar, ya que su evolución temporal muestra características de la evolución del paquete de ondas. En este apartado calculamos la función de participación en función del tiempo para ondas inicialmente extendidas, en presencia de un campo eléctrico. Cualitativamente los resultados son similares para ondas inicialmente localizadas.

En la figura 4.26 se muestra la función de participación frente al tiempo para un nanotubo de longitud $L=60 a$, y diferentes valores del campo eléctrico. Así mismo y con el fin de compararlas, se muestra el desplazamiento cuadrático medio (curvas en color rojo), en unidades arbitrarias. Como podemos ver, la curva correspondiente a la función de participación muestra numerosas irregularidades que hacen difícil su análisis. Sin embargo, sí muestra cualitativamente el mismo comportamiento que el desplazamiento cuadrático medio con el campo eléctrico. Conforme aumenta éste último, aumenta también la frecuencia de las oscilaciones y disminuye su amplitud. En las figuras 4.27 y 4.28 se muestra la función de participación en función del tiempo para dos nanotubos de longitud $L=90$ y $L=180$, respectivamente, y diferentes valores del campo eléctrico, que reproducen un comportamiento similar.

A la vista de los resultados, es obvio que en el análisis cuantitativo del fenómeno de las oscilaciones de Bloch es preferible la información que proporciona el desplazamiento cuadrático medio frente a la que proporciona la función de participación. El desplazamiento cuadrático medio reproduce las oscilaciones del paquete de ondas de manera más suave. La función de participación muestra un gran ruido debido a la difusión de la onda en la dirección transversal al campo eléctrico. Este ruido se hace comparable a la oscilación para campos eléctricos elevados debido a que al aumentar el campo decrece la amplitud, hasta el punto de que no detectamos dichas oscilaciones, ya que la señal se pierde en el ruido. Por este motivo, no tratamos de extraer información sobre la evolución del paquete de ondas mediante el radio de participación. 


\subsection{Estudio de las oscilaciones de Bloch en cavida- des}

Para completar nuestro trabajo acerca del comportamiento de las funciones de onda en nanotubos de carbono en presencia de un campo eléctrico, estudiaremos en este apartado la dinámica de las funciones de onda en nanoestructuras con cavidades sometidas a un campo eléctrico. En particular, trabajaremos con una cavidad del tipo $(6,6) / 4(9,9) /(6,6)$. Para limitar en lo posible los efectos de borde, alargamos nuestra estructura por ambos contactos hasta alcanzar una longitud cuatro veces mayor a la utilizada en el capítulo anterior, donde el nanotubo $(6,6)$ sólo poseía 2 celdas unidad.

Dada la geometría de nuestro sistema, hemos de estudiar lo efectos producidos por la aplicación del campo eléctrico en diferentes regiones de la estructura. Así, dividimos nuestro estudio en tres partes: 1) Campo eléctrico aplicado únicamente en la región central de la estructura, la cavidad. 2) Campo eléctrico aplicado en uno de los contactos y 3) Campo eléctrico aplicado en toda la estructura.

Antes de realizar los cálculos con campo eléctrico, presentamos en primer lugar la evolución temporal de ondas iniciales de Wannier en ausencia de campo eléctrico, figura 4.29. En dicha figura puede verse cómo la onda inicialmente localizada se expande sobre el nanotubo, aunque la presencia de la cavidad altera la propagación de la misma provocando una ligera discontinuidad. Es de esperar que al someter nuestra estructura a un campo eléctrico, estos efectos aumenten. Veamos los resultados obtenidos en las siguientes secciones.

\subsubsection{Campo eléctrico en el interior de la cavidad}

En este apartado vamos a estudiar la evolución de ondas de Wannier iniciales en la estructura $(6,6) / 4(9,9) /(6,6)$, cuando sometemos a la misma a un campo 


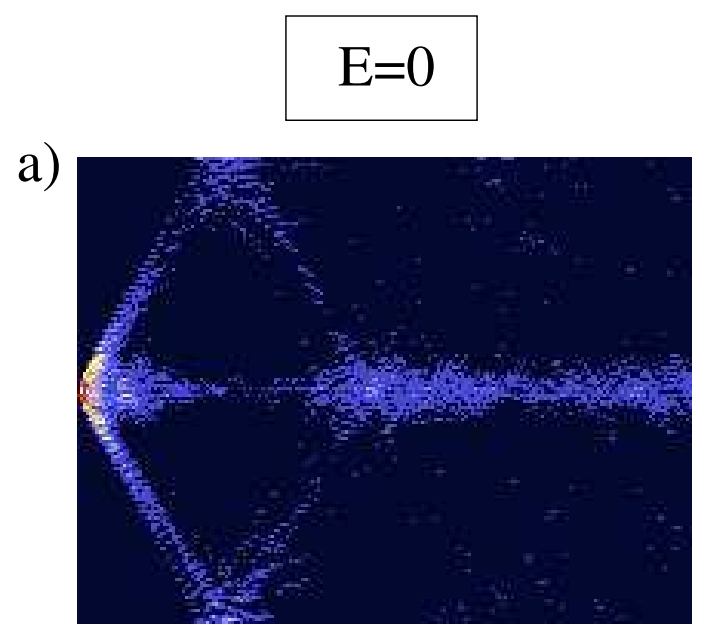

b)

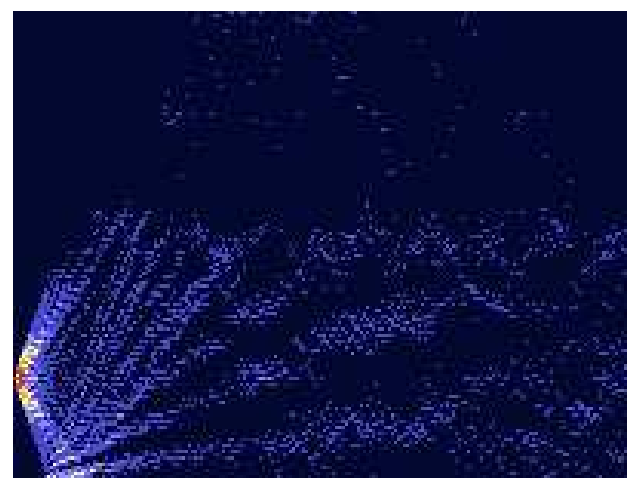

c)

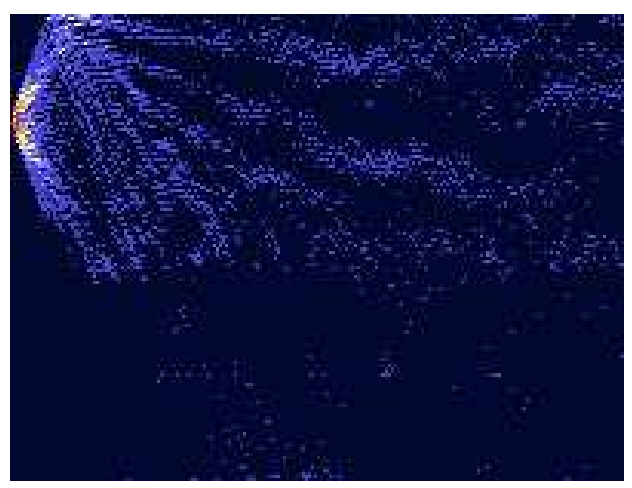

Figura 4.29: Probabilidad de encontrar al electrón en una determinada coordenada $y$ del eje del nanotubo, para ondas iniciales de Wannier en la estructura $(6,6) / 4(9,9) /(6,6)$ en función del tiempo, en ausencia de campo eléctrico. Como puede comprobarse en las tres figuras, la onda muestra su tendencia a extenderse por toda la estructura, aunque la presencia de la cavidad limita dicha expansión. La figura a) muestra la evolución de una onda inicialmente localizada en el interior de la cavidad. La b) una onda localizada inicialmente en el contacto inferior, y la c) una localizada inicialmente en el contacto superior. 
eléctrico aplicado únicamente sobre la región central de la estructura, la cavidad. Puesto que esta región central tiene un tamaño muy pequeño, no se espera en este caso la aparición de ondas de Bloch. Sin embargo, la aplicación del campo eléctrico sí podría dar lugar a fenómenos de localización de la onda.

En la figura 4.30 se representa la probabilidad de encontrar al electrón en una determinada coordenada $y$ del eje del nanotubo para ondas iniciales de Wannier, y aplicando el campo eléctrico sólo en la región central del nanotubo, esto es, en la cavidad. Las figuras a), c) y e), en la parte izquierda de la figura, se corresponden con un campo eléctrico igual a $E=813 \mathrm{MV} / \mathrm{m}$. Las figuras b), d) y f), a la derecha de la figura, se corresponden con un campo eléctrico igual a $E=4065 \mathrm{MV} / \mathrm{m}$. Se ha representado la evolución de tres ondas de Wannier iniciales en las tres regiones principales en que se divide la estructura. Las figuras a) y b) se corresponden con una onda inicialmente localizada en el contacto de la parte inferior de la estructura, de acuerdo con la representación gráfica utilizada. Las figuras c) y d) con ondas inicialmente localizadas en el centro de la cavidad, y las figuras e) y f) con ondas localizadas en el contacto superior del nanotubo.

Como puede verse en la figura 4.30, la acción del campo eléctrico en la cavidad provoca un confinamiento de la onda en las tres regiones principales en que se divide la estructura. En las subfiguras a) y b) la onda queda confinada en el contacto inferior de la estructura, región donde se encontraba la onda inicialmente. Las subfiguras c) y d) muestran el confinamiento de la onda en el interior de la cavidad, y las subfiguras e) y f) en el contacto superior. Estos efectos son mucho más claros a medida que aumenta el valor del campo eléctrico, como puede verse por comparación de las figuras correspondientes a $E=813 \mathrm{MV} / \mathrm{m}$, subfiguras a), c) y e), con las correspondientes a $E=4065 \mathrm{MV} / \mathrm{m}$, subfiguras b), d) y f). 

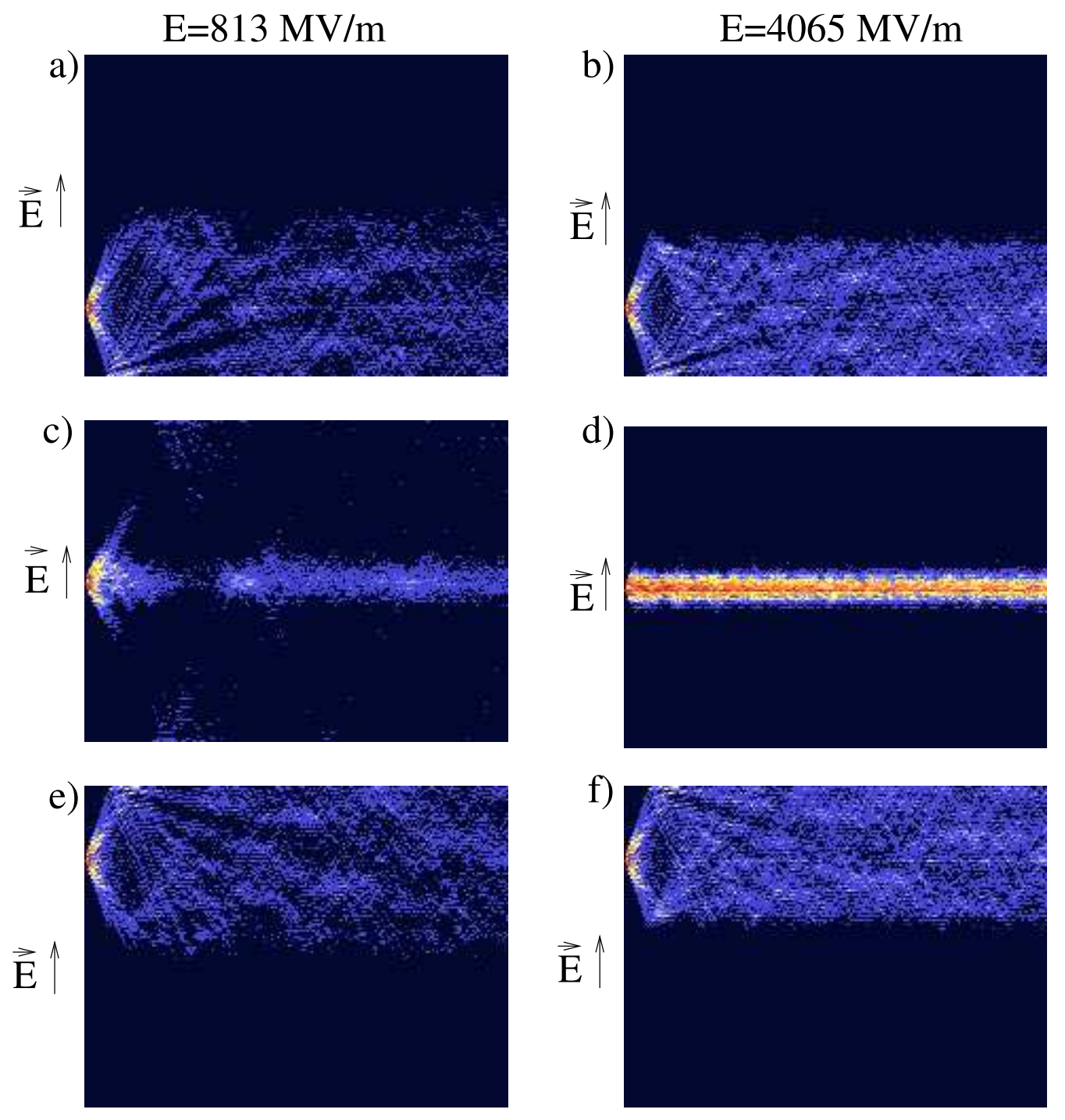

Figura 4.30: Probabilidad de encontrar al electrón en una determinada coordenada $y$ del eje del nanotubo, para ondas iniciales de Wannier en la estructura $(6,6) / 4(9,9) /(6,6)$ en función del tiempo, en presencia de un campo eléctrico aplicado únicamente en la cavidad, de valor $E=813 \mathrm{MV} / \mathrm{m}$ para las figuras representadas a la izquierda y de valor $E=4065 \mathrm{MV} / \mathrm{m}$ para las representadas a la derecha. Las figura a) y b) muestran la evolución de una onda inicialmente localizada en el contacto inferior. Las c) y d) una onda localizada inicialmente el interior de la cavidad, y las e) y f) una localizada inicialmente en el contacto superior. Como puede apreciarse en la figura, la aplicación de un campo eléctrico en la cavidad provoca el confinamiento de la onda en la región inicial. 


\subsubsection{Campo eléctrico en los contactos}

En este apartado realizaremos un estudio de la evolución de las ondas de Wannier iniciales cuando sometemos a nuestra estructura a un campo eléctrico en la región correspondiente a uno de los contactos. Hemos tomado para nuestros cálculos un campo eléctrico aplicado sobre el contacto que se representa en la parte inferior de las figuras.

En la figura 4.31 se representa la probabilidad de encontrar al electrón en una determinada coordenada $y$ del eje del nanotubo en función del tiempo, para tres ondas inicialmente localizadas cada una en una de las tres regiones diferenciadas en que se divide nuestra estructura. Hemos tomado un valor del campo eléctrico igual a $E=4065 \mathrm{MV} / \mathrm{m}$, como en las figuras b), d) y f) del apartado anterior.

Como vemos en la figura 4.31, también en este caso se produce el confinamiento de las ondas, que quedan separadas en dos regiones. Las ondas inicialmente localizadas en regiones sobre las que no actúa el campo eléctrico, figuras b) y c), se extienden a lo largo del nanotubo sin llegar a penetrar en la región donde se aplica el campo eléctrico. Igual sucede para la onda localizada en la región donde actua el campo eléctrico (figura a)), ya que dicha onda no penetra en las regiones donde no actúa el campo eléctrico. Se aprecia además en esta figura la aparición de ondas de Bloch, ya que los contactos son lo suficientemente largos como para que este fenómeno pueda apreciarse, al contrario de lo que sucedía con el campo eléctrico en la cavidad. La reflexiones en el extremo inferior de la estructura provocan que dichas oscilaciones no puedan apreciarse con claridad, fenómeno que ya apareció en el caso de nanotubos puros. 

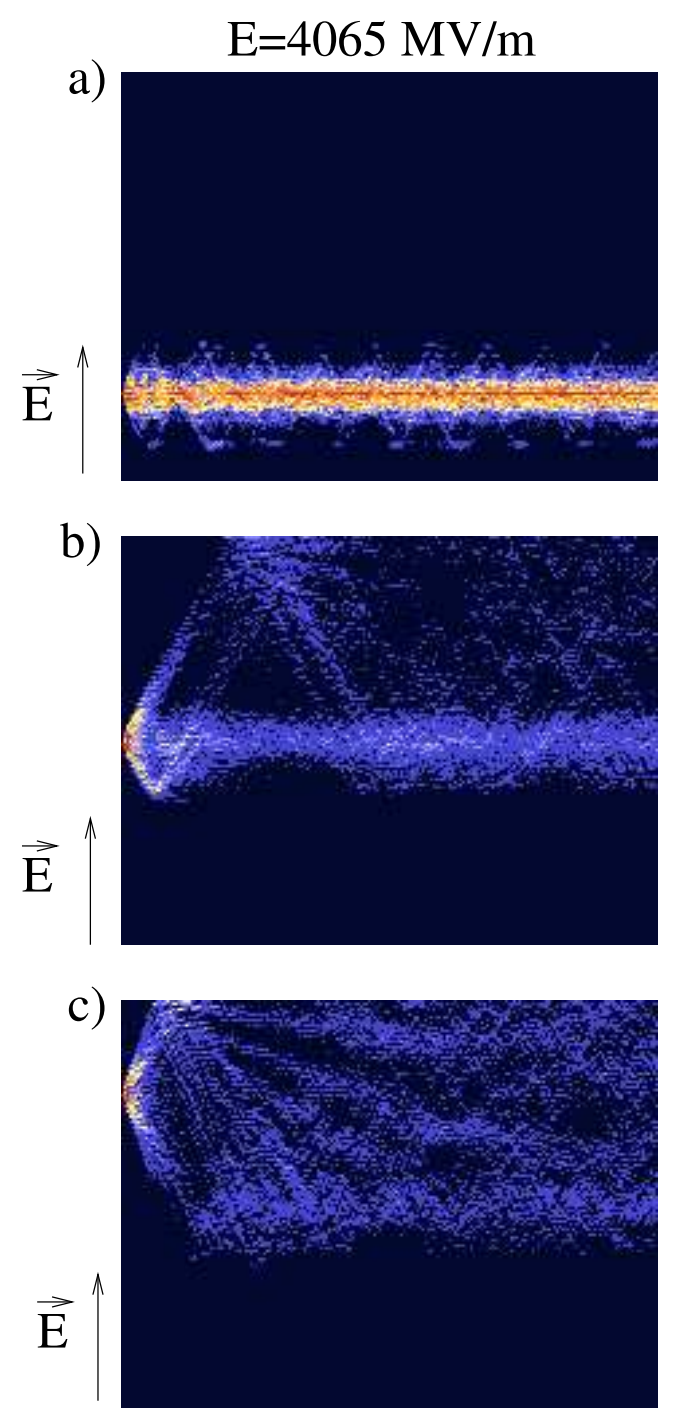

Figura 4.31: Probabilidad de encontrar al electrón en una determinada coordenada $y$ del eje del nanotubo, para ondas iniciales de Wannier en la estructura $(6,6) / 4(9,9) /(6,6)$ en función del tiempo, en presencia de un campo eléctrico de valor $E=4065 \mathrm{MV} / \mathrm{m}$ aplicado únicamente en el contacto inferior. La figura a) muestra la evolución de una onda inicialmente localizada en el contacto inferior, donde se aplica el campo eléctrico. Puede verse cómo aparecen, difuminadas, oscilaciones de Bloch. La b) muestra una onda localizada inicialmente el interior de la cavidad, que se expande únicamente hacia la región donde no existe campo eléctrico, y la c) una localizada inicialmente en el contacto superior, con parecido comportamiento a la localizada inicialmente en la cavidad. 


\subsubsection{Campo eléctrico en toda la estructura}

En este apartado estudiaremos los efectos provocados por la aplicación de un campo eléctrico en toda la estructura. Tomaremos nuevamente un valor del campo eléctrico igual a $E=4065 \mathrm{MV} / \mathrm{m}$.

En la figura 4.32 se muestra la evolución de la probabilidad de encontrar al electrón en una determinada coordenada $y$ del eje del nanotubo para ondas iniciales de Wannier en función del tiempo. Se han diferenciado nuevamente los cálculos para las tres regiones diferenciadas de nuestra estructura. En la figura a) se representan los efectos sobre una onda de Wannier localizada inicialmente en el contacto inferior del nanotubo. En la b) sobre una onda localizada inicialmente en la cavidad, y en la c) sobre una onda inicialmente localizada en el contacto superior. Se aprecia claramente en los tres casos el confinamiento de la onda en la región donde se encuentra inicialmente. Los efectos son muy claros y llaman la atención especialmente si los comparamos con la evolución de la onda en ausencia del campo eléctrico (figura 4.29. En el caso de la figura c) aparecen, además, oscilaciones de Bloch muy definidas, a diferencia de lo que sucede en la figura a). Hemos querido mostrar estas diferencias, que se deben únicamente a que en el cálculo de la figura c) se tomó una onda inicialmente localizada a mayor distancia del extremo del nanotubo.

Así pues, los resultados obtenidos mediante la aplicación de un campo eléctrico en una cavidad permiten concluir que se produce un fenómeno de confinamiento debido a dicho campo. Este fenómeno podría dar lugar a diversas aplicaciones al poder, mediante el uso de campos eléctricos, manipular la posición de los electrones en diversas regiones de estructuras formadas con nanotubos de carbono. 


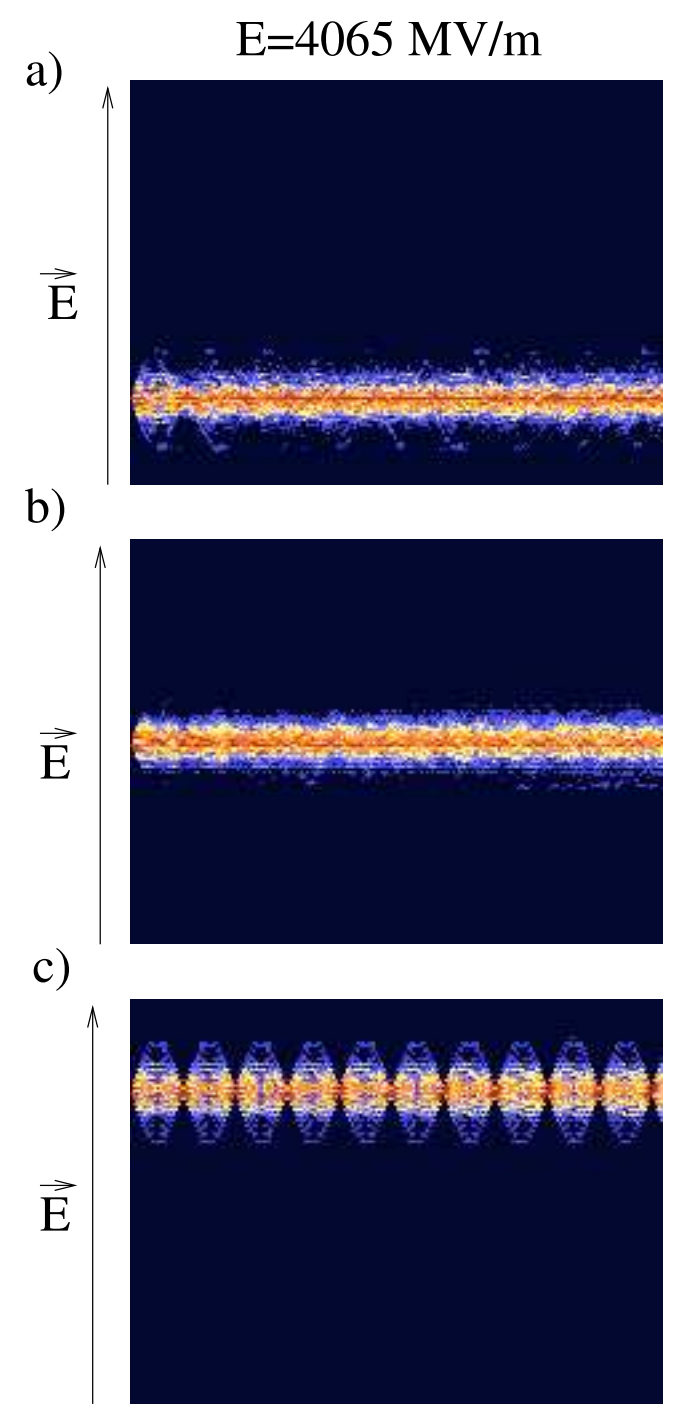

Figura 4.32: Probabilidad de encontrar al electrón en una determinada coordenada $y$ del eje del nanotubo, para ondas iniciales de Wannier en la estructura $(6,6) / 4(9,9) /(6,6)$ en función del tiempo, en presencia de un campo eléctrico de valor $E=4065 \mathrm{MV} / \mathrm{m}$ aplicado en toda la estructura. La figura a) muestra la evolución de una onda inicialmente localizada en el contacto inferior. La b) muestra una onda localizada inicialmente el interior de la cavidad, y la c) una localizada inicialmente en el contacto superior, donde se aprecian claramente oscilaciones de Bloch. En este caso se produce la localización de las tres ondas. 


\section{Conclusiones}

Mencionamos a continuación brevemente las principales aportaciones de esta tesis. Clasificaremos los resultados en dos grupos. En primer lugar mostramos los resultados y conclusiones obtenidos en relación al estudio realizado con cavidades y estrechamientos de nanotubos. En segundo lugar mostramos los resultados y conclusiones obtenidos en relación al estudio realizado sobre la aplicación de un campo eléctrico constante en nanotubos de carbono.

ESTUDIO DE CAVIDADES Y ESTRECHAMIENTOS

$\square$ Se ha desarrollado un método de cálculo eficiente para obtener las propiedades asociadas a la transmisión en nanoestructuras de carbono basado en la obtención de la función de Green del sistema.

$\square$ Se han calculado las funciones de transmisión, así como las densidades locales de estados, en diversas nanoestructuras con cavidades y estrechamientos.

$\square$ Se ha demostrado la existencia de estados cuasi-localizados en el interior de cavidades formadas por nanotubos de carbono, mediante el cálculo de la función de participación en dichos sistemas.

$\square$ Se ha evidenciado la existencia de reflexiones en las uniones entre nanotubos que forman las cavidades, encontrando una relación entre la formación de

resonancias en el interior de las cavidades con la longitud y anchura de las 
mismas. En concreto, se ha encontrando que a medida que aumenta la longitud de la cavidad, aumenta el número de resonancias en la región de Fermi del sistema. Así mismo, a medida que aumenta la anchura de la cavidad, aumenta también el número de resonancias en dicha región.

$\square$ Se ha estudiado el comportamiento de las cavidades formadas por semiconductores, demostrando la formación de puntos cuánticos.

$\square$ Se ha evidenciado la transmisión por efecto túnel en estrechamientos de nanotubos, así como se ha demostrado la inexistencia de estados localizados en el interior de los mismos, en oposición de lo que sucede en las cavidades.

$\square$ Se ha estudiado la relación entre la separación de las cavidades con la producción de resonancias en la región de Fermi, encontrando un acercamiento entre las mismas a medida que aumenta la separación entre las cavidades.

$\square$ Se ha estudiado la evolución de la transmisión a medida que aumenta el número de cavidades en un sistema, calculando finalmente la función de transmisión en una red de infinitas cavidades.

\section{CAMPo ElÉCtRico En nanotubos de CARBono}

$\square$ Se ha desarrollado un método de cálculo eficiente para obtener las propiedades dinámicas asociadas a la transmisión en nanotubos de carbono sometidos a un campo eléctrico constante, basado en el cálculo de las autofunciones de onda dependientes del tiempo.

$\square$ Se ha demostrado la existencia de oscilaciones de Bloch en nanotubos de carbono, contrastando las oscilaciones obtenidas mediante nuestro cálculo numérico con las que predice la teoría de Bloch para cadenas de átomos unidimensionales. 
$\square$ Se ha estudiado la dependencia de las oscilaciones de Bloch con la intensidad del campo eléctrico. Como predice la teoría para cadenas unidimensionales de átomos, se pone de manifiesto un aumento en la frecuencia de la oscilación con el aumento del campo eléctrico. Así mismo, la amplitud de la misma disminuye.

\ Se han obtenido el desplazamiento cuadrático medio y la función de participación en nanotubos sometidos a un campo eléctrico, realizando el cálculo de la frecuencia de la oscilación mediante el cálculo de la transformada de Fourier de dichas funciones.

$\square$ Se han estudiado las oscilaciones de Bloch producidas tanto en estados localizados como en extendidos, evidenciando el fenómeno de la localización dinámica en el primer caso, mientras que en el segundo se produce una oscilación del centro del paquete de ondas.

$\square$ Se ha realizado el estudio del comportamiento de las autofunciones de onda en cavidades de nanotubos sometidos a campos eléctricos, mostrando el fenómeno del confinamiento de la onda debido a la aplicación de dicho campo en diferentes regiones del nanotubo. 


\section{Bibliografía}

Ajayan P. M., Ebbesen T. W., Ichihashi T., Iijima S., Tanigaki K., Y HiURA H. (1993). Nature (London), 362522.

AjayAn P. M. y IIJima S. (1992). Nature (London), 35823.

Ajayan P. M. y Iijima S. (1993). Nature (London), 361333.

Andriotis A. N., Menon M., Srivastava D., y Chernozatonskit L. (2002).

Phys. Rev. Lett., 87066802.

Ashcroft N. W. y Mermin N. D. (1976). Solid State Physics. Saunders College Publishing, New York.

BANHART F. (2001). Nanoletters, 1329.

Banyai L. Y Koch S. W. (1993). Semiconductor Quantum Dots. World Scientific.

Bates K. R. y Scuseria G. E. (1998). Teor. Chem. Acc., 9929.

Blackman L. C. F. y Ubbelohde A. R. (1962). Proc. Roy. Soc. A, 26620.

Blase X., Benedict L. X., Shirley E. L., y Louie S. G. (1994). Phys. Rev. Lett., 721878.

Bloch F. (1928). Z Phys., 52555. 
Bockrath M., Obden D. H., McEuen P. L., Chopra N. G., Zettl A., Thess A., y Smalley R. E. (1997). Science, 2751922.

Chico L., Crespi V. H., Benedict L. X., Loui S. G., y Cohen M. L. (1996). Phys. Rev. Lett., 76971.

Chico L. Y Jaskolski W. (2004). Phys. Rev. B, 69085406.

Chico L., Sancho M. P. L., Y Muñoz M. C. (1998). Phys. Rev. Lett., 811278.

Choi H. J., Ihm J., Louie S. G., y Cohen M. L. (2000). Phys. Rev. Lett., 84 2917.

Collins P. G., Zettl A., Bando H., Thess A., y Smalley R. E. (1997). Nature (London), 278100.

Cowley J. M., Nikolaev P., Thess A., y Smalley R. E. (1997). Chem. Phys. Lett., 265379.

Cowley J. M. y Sundell F. A. (1997). Ultramicroscopy, 681.

Dahan M. B., Peik E., Reichel J., Castin Y., y Salomon C. (1996). Phys. Rev. Lett., 764508.

DatTa S. (1995). Electronic Transport In Mesoscopic Systems. Cambridge University Press, Cambridge.

Deepak F. L., Govindaraj A., y Rao C. N. R. (2006). J. Chem. Sci., 1189. Dekorsy T., Leisching P., Köhler K., y Kurz H. (1994). Phys. Rev. B, 50 8106.

del Valle M., Tejedor C., y Cuniberti G. (2005). Phys. Rev. B, 71125306.

Demczyk B. G., Wang Y. M., Cumings J., Hetman M., Han W., Zettl A., Y Ritchie R. O. (2002). Materials Science and Engineering A, 334173. 
Dravid V. P., Lin X., Wang Y., Wang X. K., Yee A., Ketterson J. B., Y Chang R. P. H. (1993). Science, 2591601.

Dresselhaus M. S., Dresselhaus G., Y Avouris P. (2001). Carbon Nanotubes: Synthesis, Structure, Properties, and Applications. Springer-Verlag, Berlin.

Dresselhaus M. S., Dresselhaus G., Sugihara K., Spain I. L., y GoldBERG H. A. (1988). Graphite Fibers and Filaments. Springer-Verlag, Berlin.

Dujardin E., Ebbesen T. W., Hiura H., y Tanigaki K. (1994). Science, 265 1850.

Ebbesen T. W. (1994). Annu. Rev. Mater. Sci., 24235.

Ebbesen T. W. (1997). Carbon Nanotubes: Preparation and Properties. CRC Press, Florida (USA).

Ebbesen T. W., Hiura H., Fujita J., Ochiai Y., Matsui S., y Tanigaki K. (1993). Chem. Phys. Lett., 20983.

Economou E. N. (1983). Green's Functions in Quantum Physiscs. SpringerVerlag, Berlin.

Endo M., Koyama T., y Hishiyama Y. (1976). Jap. J. Appl. Phys., 152073.

Endo M., Takeuchi K., Igarashi S., Kobori K., Shiraishi M., y Kroto H. W. (1993). J. Phys. Chem. Solids, 541841.

Endo M., Takeuchi K., Kobori K., Takahashi K., Kroto H., y Sarkar A. (1995). Carbon, 33873.

Esaki L. Y Tsu R. (1970). IBM J. Res. Dev., 1461.

Falvo M. R., Clary G. J., it R. M. T., Chi V., Jr. F. P. B., Washburn S., y Superfine R. (1997). Nature, 389582. 
Farajian A. A., Mizuseki H., y KaWazoe Y. (2004). Physica E, 22675.

Feldmann J., Leo K., Shah J., Miller D. A. B., Cunningham J. E., Meier T., von Plessen G., Schulze A., Thomas P., y Schmitt-Rink S. (1992). Phys. Rev. B, 467252.

Gal'pern E. G., Stankevich I. V., Chistykov A. L., y Chernozatonskit L. A. (1993). Chem. Phys. Lett., 214345.

Gasparian V., Gummich U., Jódar E., Ruiz J., y Ortuño M. (1997). Physica B. Condensed Matter., 23372.

Glüch M., Kolovsky A. R., y Korsch H. J. (2002). Phys. Rep., 366103.

Grecchi V. y Sacchetti S. (2001). Phys. Rep., 63212303.

Grifoni M. y HängGi P. (1998). Phys. Rep., 304229.

Grimm D., Muniz R. B., y Latgé A. (2003). Phys. Rev. B, 68193407.

Gülseren O., Yildirim T., Ciraci S., Y Ç. Kiliç (2002). Phys. Rev. B, 65 155410 .

Hartman T., Keck F., Korsch H. J., y Mossmann S. (2004). New J. Phys., 62.

Herkel T., Martel R., y Avouris P. (1995). Adv. Mater., 7275.

Hiura H., Ebbesen T. W., y Tanigaki K. (1995). Adv. Mater., 7275.

Ignatov A. A., Schomburg E., Renk K. F., Schatz W., Palmier J. F., Y Mollot F. (1994). Ann. Physik, 3137.

IIJIMA S. (1991). Nature (London), 35456.

IIJima S. (1993). Mater. Sci. Eng. B, 19172. 
IıJima S. (1998). Fullerenes and Nanotubes. Pierre Delhaès and P. M. Ajayan, Gordon and Breach Publishers., Paris.

Ikazaki F., Oshima S., Uchida K., Kuriki Y., Hayakawa H., Yumura M., Takahashi K., Y TOJima K. (1994). Carbon, 321539.

Jin Y. y Yuan F. G. (2003). Composites Science and Technology, 631507.

Jódar E. y Pérez-Garrido A. (2007). Phys. Stat. Sol. (a), 2041892.

Jódar E., Pérez-Garrido A., y Díaz-Sánchez A. (2006). Phys. Rev. B, 73 205403.

Jódar E., Pérez-Garrido A., y Rojas F. (2007a). Phys. Stat. Sol. (a) (Enviado).

Jódar E., Pérez-Garrido A., y Rojas F. (2007b). Phys. Rev. Lett. (Enviado).

Journet C., Maser W. K., Bernier P., Loiseau A., De la Chapelle M. L., Lefrant S., Deniard P., Lee R., y Fischer J. E. (1997). Nature (London), 388756 .

Kataura H., Kimura A., Ohtsuka Y., Suzuki S., Maniwa Y., Hanyu T., Y Achiba Y. (1998). Jpn. J. Appl. Phys., 37 L616.

KitTel C. (1986). Introduction to Solid State Physics. John Wiley and Sons, New York.

Kostyrko T., Bartkowiak M., y Mahan G. D. (1999a). Phys. Rev. B, 59 3241.

Kostyrko T., Bartkowiak M., y Mahan G. D. (1999b). Phys. Rev. B, 60 10735 .

Koyama T. (1972). Carbon, 10757. 
Krätschmer W., Lamb L. D., Fostiropoulus K., y Huffman D. (1990). Nature, 347354.

Kroto H. W., Heath J. R., O’Brien S. C., Curl R. F., y Smalley R. E. (1985). Nature (London), 318162.

Leisching P., Bolivar P. H., Beck W., Dhaibi Y., BrÜggemann F., Schwedler R., Kurz H., Leo K., Y Köhler K. (1994). Phys. Rev. B, 50 14389.

Leo K., Bolivar P. H., Brüggemann F., Schwedler R., y Köhler K. (1992). Solid State Comm., 84943.

Li C. y Chou T. W. (2003). International Journal of Solids and Structures, 40 2487.

Li C., Papadopoulos C., Y Xu J. (1999). Nature (London), 402254.

Mendez E. E., Agullo-Rueda F., y Hong J. M. (1988). Phys. Rev. Lett., 60 2426 .

Menon M., Andriotis A. N., Srivastava D., Ponomareva I., y ChernoZatonskit L. A. (2003). Phys. Rev. Lett., 91145501.

Menon M. y Srivastava D. (1997). Phys. Rev. Lett., 794453.

Moore A. W., Ubbelohde A. R., y Young D. A. (1962). Brit. J. Appl. Phys., 13393.

Nazareno H. N., Da Silva C. A. A., y de Brito P. E. (1994). Phys. Rev. B, 504503.

Nazareno H. N. y de Brito P. E. (2001). Phys. Rev. B, 64045112.

Oberlin A., Endo. M., y Koyama T. (1976a). Carbon, 14133. 
Oberlin A., Endo. M., y Koyama T. (1976b). Phys. J. Crystal Growth, 32 335 .

Orlikowski D., Mehrez H., Taylor J., Guo H., Wang J., y Roland C. (2001). Phys. Rev. B, 63155412.

Osawa E. (1970). Kagaku, 25854.

Pérez-Garrido A. (2002). J. Phys. Condens. Matter, 145077.

Pérez-Garrido A., Alhama F., y Catalá J. D. (2002). Chem. Phys., 278 71.

Pérez-Garrido A. y Urbina A. (2002). Carbon, 401227.

Pertsch T., Dannberg P., Elflein W., Bräuer A., y Lederer F. (1999). Phys. Rev. Lett., 834752.

Rocha C. G., Dargam T. G., y Latgé A. (2002). Phys. Rev. B, 65165431.

Ruiz J., Gasparian V., Y Jódar E. (2007a). Proceedings of the International Conference on Semiconductor Micro and Nano-electronics (Aceptado).

Ruiz J., Jódar E., y Gasparian V. (2007b). Phys. Rev. B, 75235123.

Saito R., Dresselhaus G., y Dresselhaus M. S. (1996). Phys. Rev. B, 53 2044.

Saito R., Dresselhaus G., y Dresselhaus M. S. (1998). Physical Properties of Carbon Nanotubes. Imperial College Press, London.

Saito R., Dresselhaus G., y Dresselhaus M. S. (2000). Phys. Rev. B, 61 2981.

Saito Y. y Inagaki M. (1993). Jpn. J. Appl. Phys., 32 L954. 
Sammalkorpi M., Krasheninnikov A., Kuronen A., Nordlund K., y KasKI1 K. (2004). Phys. Rev. B, 70245416.

Satishkumar B. C., Thomas P. J., Govindaraj A., y Rao C. N. R. (2000). Appl. Phys. Lett., 772530.

SchwaBl F. (2001). Quantum Mechanics. Springer-Verlag, Berlin.

Seraphin S., Zhou D., Jiao J., Withers J. C., y Loufty R. (1993). Carbon, 31685.

Sim H.-S., Park C.-J., y Chang K. J. (2001). Phys. Rev. B, 63073402.

Song H.-F., Zhu J.-L., Y Xiong J.-J. (2002). Phys. Rev. B, 65085408.

Suenaga K., Colliex C., Demoncy N., Loiseau A., Pascard H., Y WiLlaime F. (1997). Science, 278653.

Tamura R. y Tsukada M. (1994). Phys. Rev. B, 497697.

Tamura R. y Tsukada M. (1999). Phys. Rev. B, 618548.

Tans S. J., Verschueren A. R. M., y Dekker C. (1998). Nature (London), 39349.

Tenne R., Homyonfer M., y Feldman Y. (1998). Chem. Mater., 103225.

Terrones M., Banhart F., Grobert N., Charlier J. C., Terrones H., Y Ajayan P. M. (2002). Phys. Rev. Lett., 890755505.

Terrones M., Hsu W. K., Terrones H., Zhang J. P., Ramos S., Jare J. P., Castillo R., Prassides K., Cheetham A. K., Kroto H. W., y Walton D. R. M. (1996). Chemical Physics Letters, 259568. 
Thess A., Lee R., Nikolaev P., Dai H., Petit P., Robert J., Xu C., Lee Y. H., Kim S. G., Rinzler A. G., Colbert D. T., Scuseria G. E., Tománek D., Fischer J. E., y Smalley R. E. (1996). Science, 273483.

Tibietts G. G. (1983). Appl. Phys. Lett., 42666.

Tibietts G. G. (1984). J. Crystal Growth, 66632.

Tsang S. C., Harris P. J. F., y Green M. L. H. (1993). Nature (London), 362522.

TurTon R. (1995). The Quantum Dot: a Journey into the Future of Microelectronics. Oxford University Press, US.

UgarTe D. (1992). Nature, 359707.

Unterrainer K., Keay B. J., Wanke M. C., Allen S. J., Leonard D., Medeiros-Ribeiro G., Bhattacharya U., y Rodwell M. J. W. (1996). Phys. Rev. Lett., 762973.

Voisin P., Bleuse J., Bouche C., Gaillard S., Alibert C., y Regreny A. (1988). Phys. Rev. Lett., 611639.

WANnier G. H. (1969). Phys. Rev., 117432.

Waschke C., Roskos H. G., Schwedler R., Leo K., Kurz H., y Köhler K. (1993). Phys. Rev. Lett., 703319.

Wegner Z. J. (1980). Z. Phys. B, 36209.

Wildöer J. W. G., Venema L. C., Rinzler A. G., Smalley R. E., y DeKKER C. (1998). Nature (London), 39159.

Witthaut D., Keck F., Korsh H. J., y Mossmann S. (2004). New J. Phys., 641. 
Yao Z., Postma H. W. C., Balents L., y Dekker C. (1999). Nature (London), 402273.

Yi J. Y. y Bernholc J. (1992). J. Chem. Phys., 968634.

Yu M.-F., Files S., Arepalli S., y Ruoff R. S. (2000a). Phys. Rev. Lett., 84 5552.

Yu M.-F., Kowalewski T., y Ruoff R. S. (2000b). Phys. Rev. Lett., 851456.

Zener C. (1934). Proc. R. Soc. London Ser. A, 1451523.

Zhou O., Fleming R. M., Murphy D. W., Chen C. H., Haddon R. C., Ramírez A. P., y Glarum S. H. (1994). Science, 2631744.

Zolyomi V. Y KÜrTi J. (2004). Phys. Rev. B, 70 085403(R). 